\title{
Molecular insights into the Tau-actin interaction
}

\author{
Dissertation \\ for the award of the degree \\ "Doctor rerum naturalium” \\ of the Georg-August-Universität Göttingen
}

within the doctoral program: "Biomolecules: Structure - Function - Dynamics"

of the Georg-August University School of Science (GAUSS)

Submitted by

Yunior Cabrales Fontela

from Granma, Cuba

Göttingen 2017 


\section{Thesis Committee}

Prof. Dr. Markus Zweckstetter, NMR-based Structural Biology, Max Planck Institute for Biophysical Chemistry

Prof. Dr. Claudia Steinem, Institute for Organic and Biomolecular Chemistry, Georg-August University of Göttingen

Dr. Jochen Hub, Computational Molecular Biophysics, Georg-August University of Göttingen

\section{Members of the examination board}

Referee: Prof. Dr. Markus Zweckstetter, NMR-based Structural Biology, Max Planck Institute for Biophysical Chemistry

$2^{\text {nd }}$ Referee: Prof. Dr. Claudia Steinem, Institute for Organic and Biomolecular Chemistry, Georg-August University of Göttingen

\section{Further members of the Examination Board}

Prof. Dr. Marina Bennati, Electron-Spin Resonance Spectroscopy, Max Planck Institute for Biophysical Chemistry

Prof. Dr. Henning Urlaub, Bioanalytical Mass Spectrometry, Max Planck Institute for Biophysical Chemistry

Prof. Dr. Tiago Fleming Outeiro, Neurodegeneration and Restaurative Research, University Medical Center Göttingen

Date of the oral examination: May 22th, 2017 


\section{Affidavit}

I hereby declare that the thesis "Molecular insights into the Tau-actin interaction" has been written independently and with no other sources and aids than quoted.

Yunior Cabrales Fontela

Göttingen, the $31^{\text {th }}$ of March 2017 


\section{Dedication}

I dedicate this thesis to Rocío, my wife, who have been a great source of inspiration and because she left everything behind to support me. To my father, because even though he doesn't know much about my professional life, he has always inspired me to go ahead and never stop. 


\section{Acknowledgements}

After four years of dedication this trip across the PhD studies has come to the end. This would not have been possible without the support and advices of several people that, with no doubts, must be mentioned.

I would like to acknowledge to my supervisor the Prof. Dr. Markus Zweckstetter for giving me the opportunity to work in this wonderful project, for his full support and motivation in conducting my scientific research. Thanks to him I have improved my skills in publishing manuscripts and writing scientific reports.

I would like to thank to the PhD thesis committee members Prof. Dr. Claudia Steinem and Dr.

Jochen Hub to accept being part of my thesis committee and for the useful discussions and advises during the meetings. Special thanks go to Prof. Dr. Marina Bennati, Prof. Dr. Henning Urlaub, and Prof. Dr. Tiago Fleming Outeiro for kindly participating in the examination committee.

I appreciate the effort of the secretary of my department Petra Breiner for all the paperwork and as a mediator in situations I faced as a foreigner.

I would like to thank to the Dr. Nasrollah Rezaei-Ghaleh who was my reference for the NMR methodology at the beginning.

I owe huge debt of gratitude to Dr. Harindranath Kadavath and Dr. Javier Oroz to be not only my friends but also for all their support, advices and discussions about experimental procedures and data analysis. I take the opportunity to express my thankfulness to the colleagues Luis Fonseca Ornelas, Aldo Román Camacho Zarco, Timo Strohäker and Filippo Favretto who have been responsible for the nice environment in our group. 


\begin{abstract}
Tau protein is classically considered as a neuronal microtubule-associated protein that stabilizes microtubules and supports the outgrowth of axons. The protein can modulate the transport of vesicles and organelles along microtubules, serves as an anchor for enzymes, and regulates the dynamics of microtubules. Tau is an intrinsically disordered protein, which becomes excessively phosphorylated in Alzheimer's disease, loses its ability to bind to microtubules and forms neurofibrillary tangles.
\end{abstract}

Similar to microtubules, actin is another important cytoskeletal protein, which is involved in generation and maintenance of cell morphology and polarity, cell division, contractility, motility, and intracellular trafficking. Microtubule-associated proteins not only regulate microtubule dynamics but bundle actin filaments and cross-link actin filaments with microtubules. In addition, aberrant interaction of the microtubule-associated protein Tau with filamentous actin is connected to synaptic impairment in Alzheimer's disease. Furthermore Hirano bodies, which are actin-rich inclusions, are found in brain histopathological samples of Alzheimer's disease and related tauopathies.

Although the interaction between Tau and actin has been studied, the nature of the interaction and molecular mechanism involved are still unclear. The intrinsically disordered nature of Tau in solution as well as the dynamic nature of Tau-actin interaction, where even in the bound state part of Tau remains flexible, limits the use of X-ray crystallography to investigate the structure of Tau bound to actin. Therefore we decided to use Nuclear Magnetic Resonance spectroscopy well as other biochemical and biophysical methods to understand the nature of interaction between Tau and actin.

Here we provide insight into the nature of interaction between actin filaments and Tau proteins and the actin-microtubule crosstalk. We show that Tau uses several short helical segments to bind in a dynamic, multivalent process to the hydrophobic pocket of actin. Although a single Tau helix is sufficient to bind to filamentous actin, at least two, flexibly linked $\alpha$-helices are required for actin bundling. In agreement with a structural model of Tau repeat sequences in complex with actin filaments, phosphorylation at serine 262 attenuates the binding of Tau to filamentous actin. Taken together the data demonstrate that bundling of filamentous actin and cross-linking of the cellular cytoskeleton depends on the metamorphic and multivalent nature of microtubuleassociated proteins. 


\section{Table of Contents}

\section{List of Tables.}

List of Figures

Abbreviations .......................................................................

1. Introduction ................................................................. 1

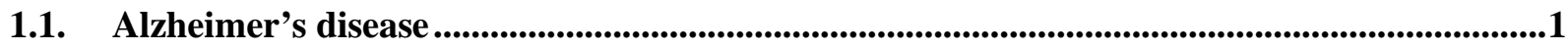

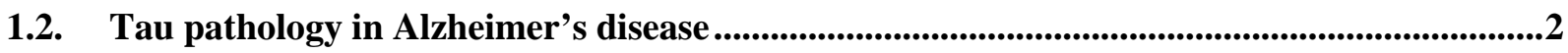

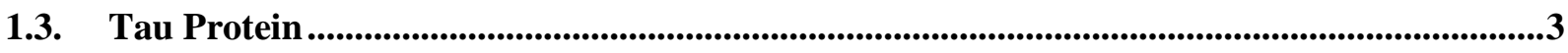

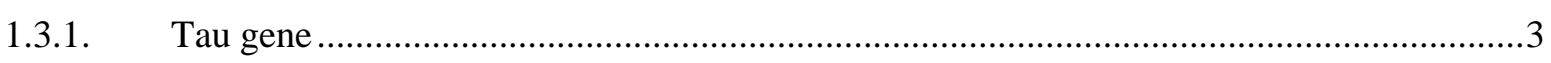

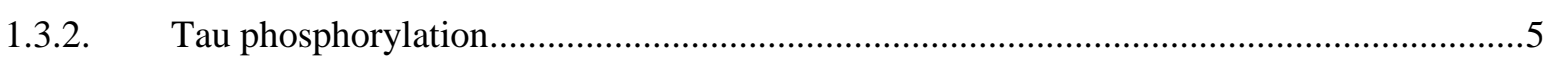

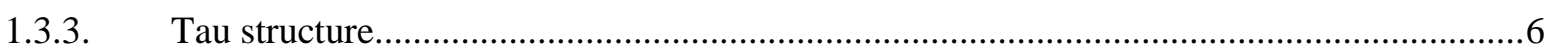

1.3.4. Tau functions................................................................................................

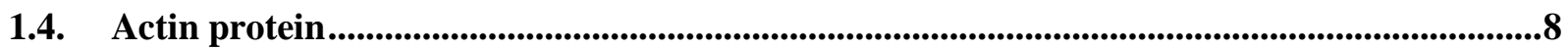

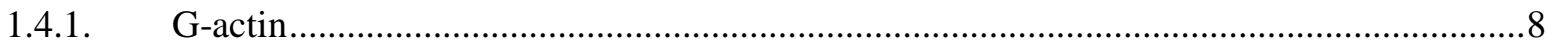

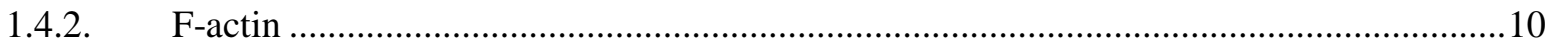

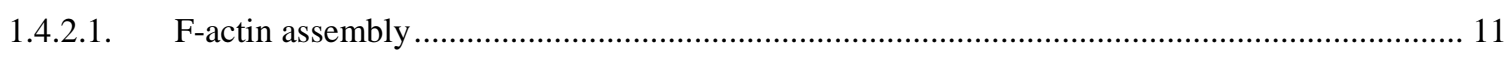

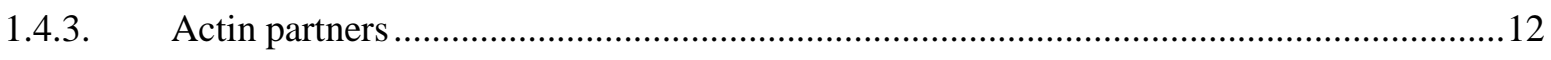

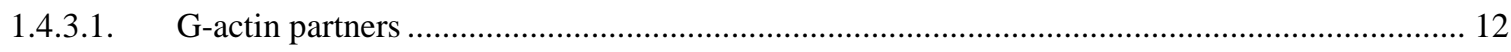

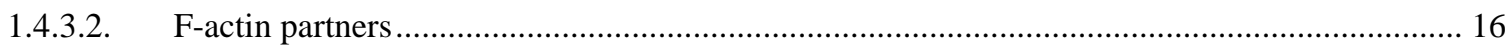

1.4.4. F-actin stability and neurodegeneration ...............................................................20

1.5. Tau and actin interaction .....................................................................................22

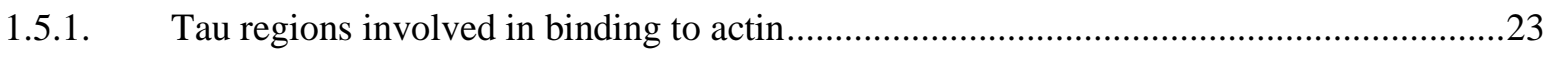

1.6. Tau-mediated microtubule F-actin interaction ..........................................................24

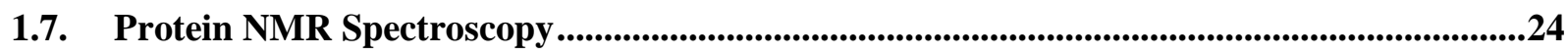

1.7.1. Relevance of structure determination using NMR ..............................................25

1.7.2. NMR-based Structure determination ............................................................25

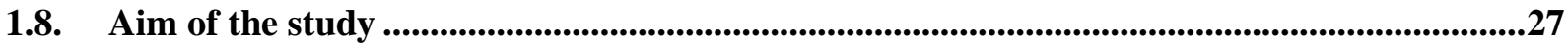

2. Materials and Methods..................................................... 28

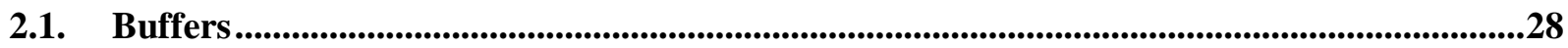

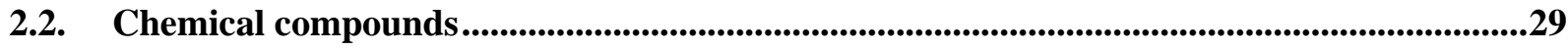

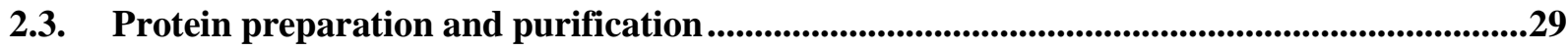

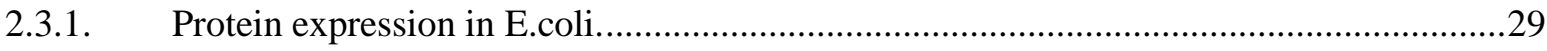

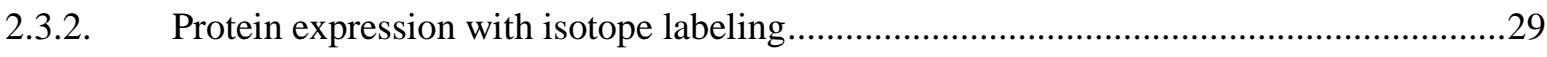


2.3.3. Standard 5 x M9 minimal medium without nitrogen source............................................. 30

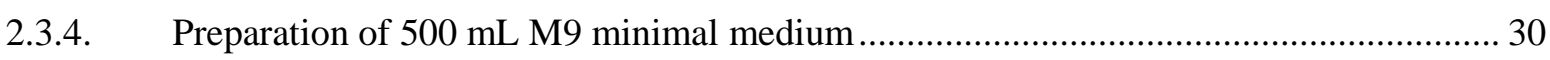

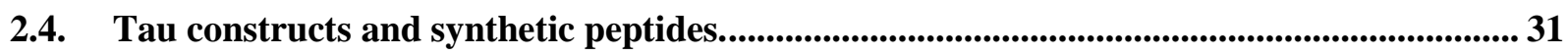

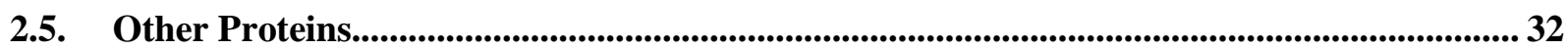

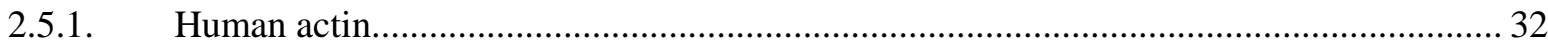

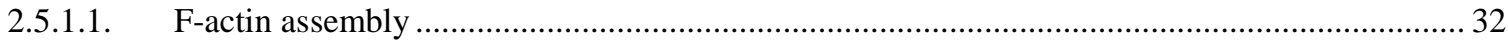

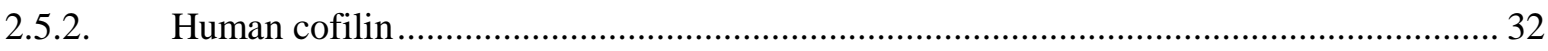

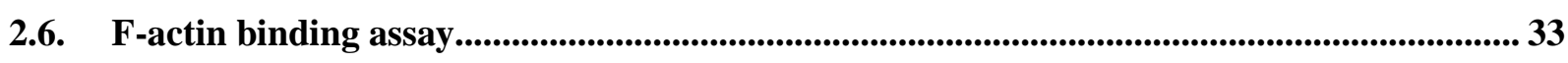

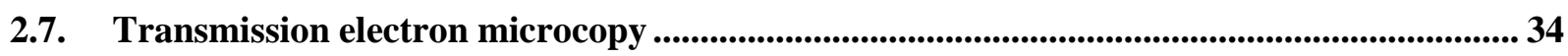

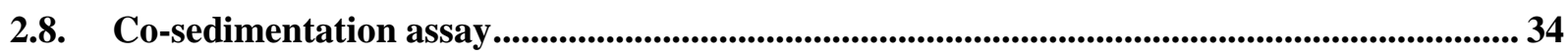

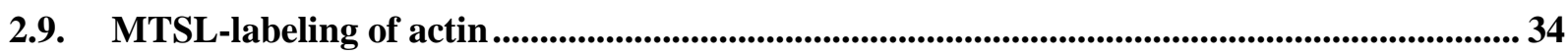

2.10. NMR spectroscopy …....................................................................................................................... 35

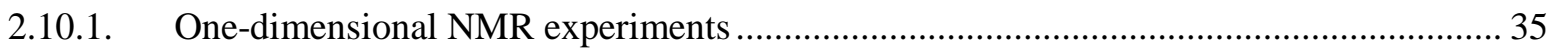

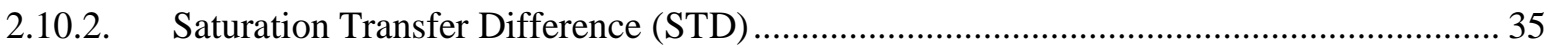

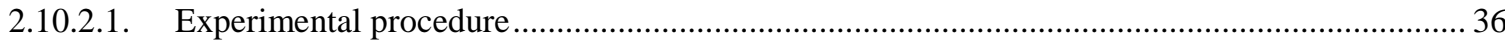

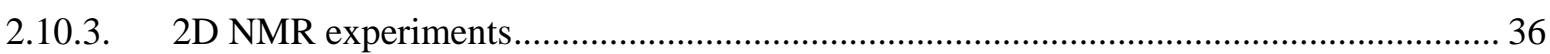

2.10.3.1. Heteronuclear Single Quantum Coherence (HSQC) …………................................................ 36

2.10.3.2. Total Correlation Spectroscopy (TOCSY) ................................................................................. 38

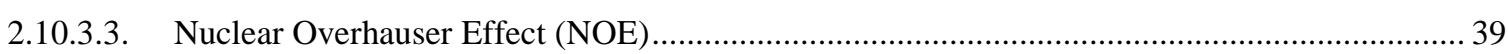

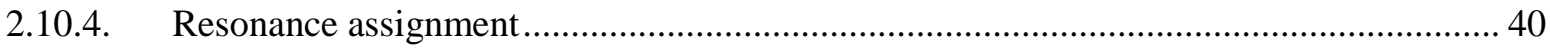

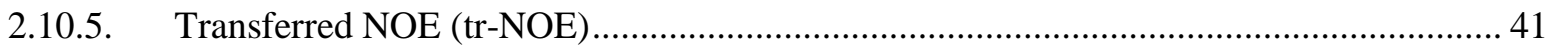

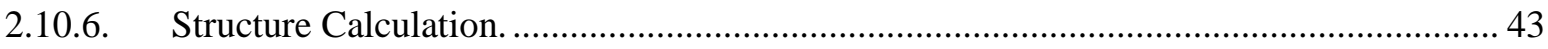

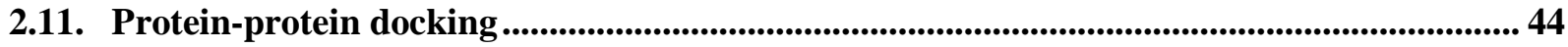

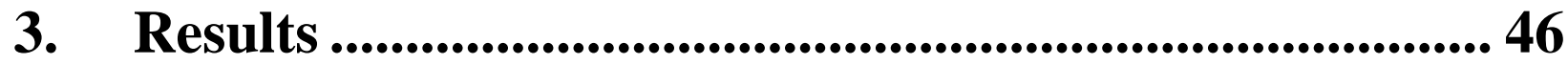

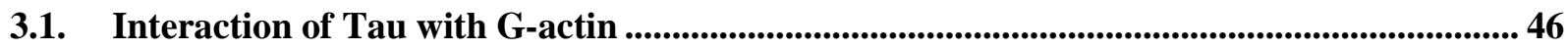

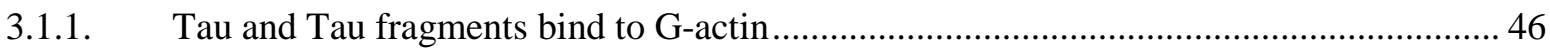

3.1.2. Identification of binding regions of Tau in complex with G-actin .................................... 47

3.1.3. Tau fragments compete with Tau for binding to G-actin ................................................ 50

3.1.4. Mapping the binding of Tau on G-actin surface............................................................. 52

3.1.4.1. Tau does not bind on the nucleotide-binding pocket of G-actin .................................................. 52

3.1.4.2. Tau binds to the hydrophobic pocket of G-actin.................................................................... 53

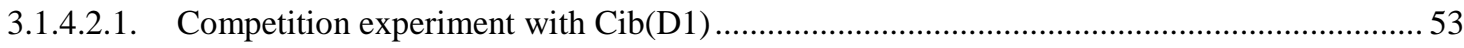

3.1.4.2.2. Further evidences form the binding of Tau on the hydrophobic pocket of G-actin............... 56

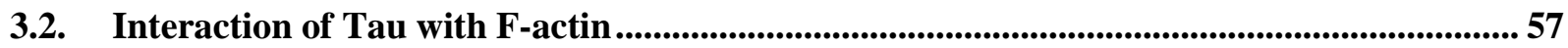

3.2.1. Tau interacts with and promotes the bundles of actin filaments...................................... 58

3.2.2. NBD-fluorescence reveals strong binding between Tau and F-actin ............................... 59

3.2.3. Identification of binding regions of Tau in complex with F-actin..................................... 60

3.2.4. Short Tau fragments from the MBD also promote F-actin bundles formation.................. 62 
3.2.5. Tau binds to the hydrophobic pocket of F-actin.........................................................64

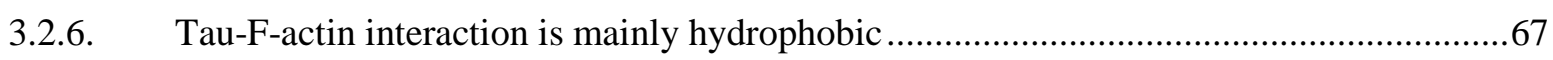

3.2.7. Tau phosphorylation affects the interaction between Tau and F-actin............................68

3.3. NMR-based structure calculation of Tau in complex with F-actin .........................................70

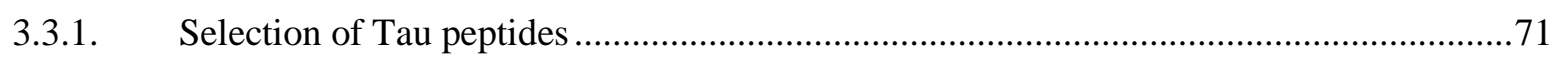

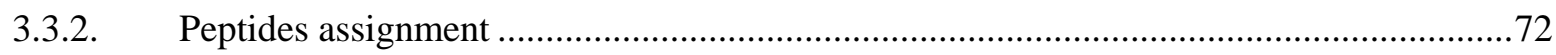

3.3.3. Structure calculation of $\operatorname{Tau}(254-268)$ bound to F-actin....................................................72

3.3.4. Structure calculation of $\operatorname{Tau}(254-290)$ bound to F-actin.................................................76

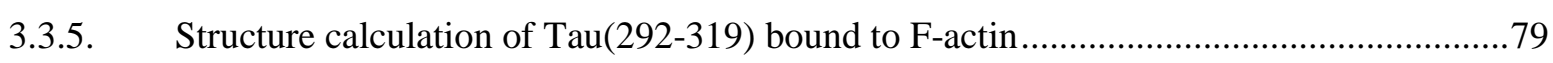

3.4. Docking the Tau(254-290) structure on F-actin surface......................................................82

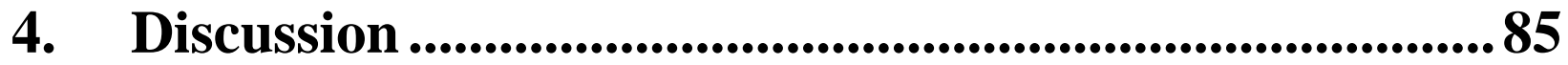

4.1. The interaction of Tau with actin ................................................................................................85

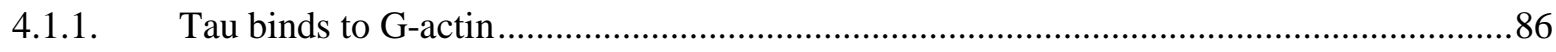

4.1.2. The MBD and proline-rich region of Tau interact with G-actin .....................................86

4.1.3. Different Tau motifs bind to the same region of G-actin .................................................87

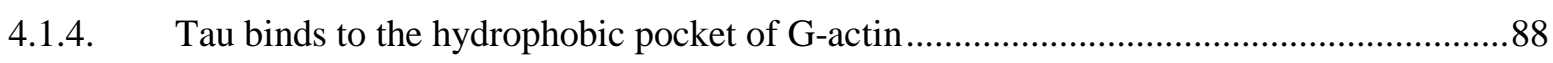

4.1.5. Influence of phosphorylation of Tau at KXGS motifs on the interaction with G-actin ....89

4.2. Relevance of the interaction between Tau and G-actin .......................................................90

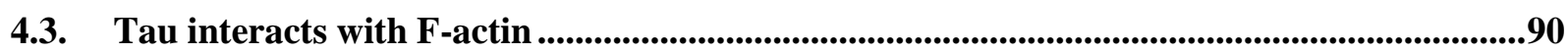

4.4. Tau binds to F-actin in a similar fashion than to G-actin: as seen by NMR ........................91

4.5. Phosphorylation of Tau affects its binding to F-actin.................................................................92

4.6. Insights into the structure of Tau bound to F-actin ......................................................................93

4.6.1. Tau peptides recognizes F-actin by means of an alpha helical motifs ............................95

4.6.2. Rolle of Tau in the cytoskeletal network organization...................................................96

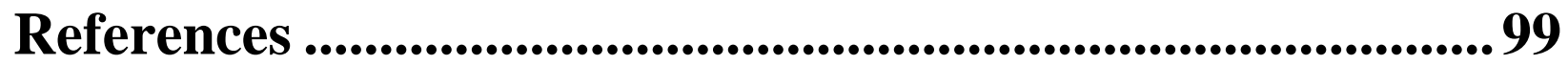




\section{List of Tables}

Table 2.1. Names and descriptions of all the buffers used in this study. 28

Table 2.2. Script used for structure calculation with a simulated annealing algorithm...... 44

Table 3.1. Structural statistics of Tau(254-268) bound to F-actin. 74

Table 3.2. Structural statistics of Tau(254-290) bound to F-actin. 78

Table 3.3. Structural statistics of Tau(292-319) bound to F-actin. 80

Table 3.4. Statistic parameters from the lower score cluster from docking. 83 


\section{List of Figures}

Figure 1.1. Progressive degeneration from neuronal to cerebral cortex....................................................1

Figure 1.2. Microtubule disassembly and formation of neurofibrillary tangles.......................................2

Figure 1.3. Schematic representation of the human Tau gene and the six Tau isoforms expressed in the

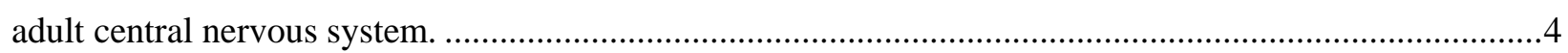

Figure 1.4. Comparison between ATP- and ADP-bound actin crystal structures........................................9

Figure 1.5. The three different phases of actin polymerization..............................................................11

Figure 1.6. Crystal structure of actin in complex with ciboulot...............................................................13

Figure 1.7. Small molecules and their complexes with G-actin................................................................15

Figure 1.8. Crystal structure of G-actin in complex with gelsolin ............................................................17

Figure 1.9. Concentration-dependent activity of cofilin. .........................................................................18

Figure 1.10. 3D structures of cofilin in complex with F-actin and in solution. .........................................19

Figure 1.11. Actin organization in neuronal spines and filopodium. ......................................................21

Figure 2.1. Tau protein and Tau constructs used in this study.................................................................31

Figure 2.2. Schematic representation of STD NMR spectroscopy...........................................................36

Figure 2.3. Schematic representation of a 2D HSQC pulse sequence......................................................37

Figure 2.4. Schematic representation of a NOESY pulse sequence...........................................................40

Figure 2.5. Pictorial representation of a fast exchange ligand-receptor interaction....................................41

Figure 3.1. Fluorescence-based analysis of NBD-labeled G-actin bound to Tau and Tau constructs. .......47

Figure 3.2. $2 \mathrm{D}{ }^{1} \mathrm{H}-{ }^{15} \mathrm{~N}-\mathrm{HSQC}$ spectra of hTau 40 in presence (red) and absence (grey) of G-actin............48

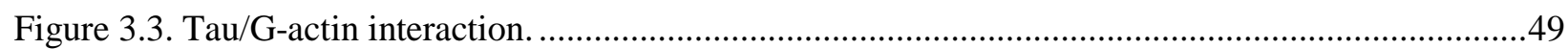

Figure 3.4. Combined chemical shift perturbation of Tau bound G-actin from ${ }^{1} \mathrm{H}^{15}{ }^{15} \mathrm{~N}-\mathrm{HSQC} \ldots \ldots \ldots \ldots \ldots \ldots . . . . .49$

Figure 3.5. Competition experiments between Tau and Tau fragments for binding to G-actin..................51

Figure 3.6. Tau does not compete with LatB for binding to G-actin on the nucleotide-binding pocket.....52

Figure 3.7. Tau and ciboulot shares the binding site on G-actin surface. .................................................54

Figure 3.8. STD-based competition experiment between Cib and Tau fragments for binding to G-actin..55

Figure 3.9. The PRE approach reveals that Tau binds nearby the hydrophobic pocket of G-Actin. ..........57

Figure 3.10. Negatively stained electron micrograph from Tau-promoted F-actin bundles. ......................58

Figure 3.11. NBD-fluorescence reveals a strong binding between Tau and F-actin...................................60

Figure 3.12. Superposition of ${ }^{1} \mathrm{H}-{ }^{15} \mathrm{~N}$ HSQC spectra of hTau40 in absence (gray) and presence (blue) of

F-actin.

Figure 3.13. Residue-specific changes in ${ }^{1} \mathrm{H}^{15}{ }^{15} \mathrm{HSQC}$ signal intensities of Tau upon addition of F-actin.

Figure 3.14. Electron micrographs from F-actin in complex with Tau peptides......................................63

Figure 3.15. Affinities of Tau and different Tau constructs for binding to NBD-labeled F-actin. 64 
Figure 3.16. Tau competes with cofilin for binding to F-actin. 65

Figure 3.17. SDS gel-based competition effect between Tau and cofilin for binding to F-actin. 65

Figure 3.18. The PRE approach reveals that Tau binds nearby the hydrophobic pocket of F-actin 66

Figure 3.19. Salt titration experiment of Tau in presence of two-fold excess of F-actin. 67

Figure 3.20. MARK2 phosphorylation decreases Tau’s affinity for F-actin. 69

Figure 3.21. SDS-PAGE of phosphorylated and unphosphorylated Tau(254-284) in presence of F-actin.70

Figure 3.22. Tau(254-290) bind to F-actin in a fast exchange regime. 71

Figure 3.23. Resonance assignment of Tau peptides. 72

Figure 3.24. Superimposed NOE spectra from Tau(254-268) in absence (blue) and in presence (red) of Factin. 73

Figure 3.25. Distribution of the NOE interactions of Tau(254-268) bound to F-actin. 75

Figure 3.26. Structure of Tau(254-268) bound to F-actin. 75

Figure 3.27. NOE intensity buildup curve for two NOE cross peaks. 77

Figure 3.28. Distribution of the NOE interactions for Tau(254-290). 78

Figure 3.29. Ribbon representation of the best 10 conformers from Tau(254-290)...... 79

Figure 3.30. Distribution of the NOE interactions for Tau(292-319)........................................... 81

Figure 3.31. Ribbon representation of the best 10 conformers from Tau(292-319)............................. 81

Figure 3.32. Active residues on F-actin used for docking calculation.............................................. 82

Figure 3.33. Superposition of 3D structures of both cofilin and Tau(250-290) in complex with F-actin. . 83

Figure 4.1. Pictorial representation of Tau/G-actin interaction. 88

Figure 4.2. Schematic representation of the importance of MAPs for the cellular cytoskeleton. 94

Figure 4.3. Mechanistic model of the Tau-induced F-actin bundles. 96

Figure 4.4. Sequence alignment of the repeat domains of MAP2c and Tau. 97

Figure 4.5. Mechanistic model of Tau-mediated F-actin/microtubule network formation. 98 


\section{Abbreviations}

$\mathrm{A} \beta$

$\mathrm{AD}$

ADP

ADF

ARP2/3

ATP

BEB

CAMKII Calmodulin-dependent Protein Kinase II

CCR Cross-correlated Relaxation

CD Cytochalasin D

cdk5 Cyclin-dependent Kinase 5

Cib Ciboulot

Cryo-EM Cryo-electron Microscopy

DTT Dithiothreitol

EB Elution buffer

EM Electron Microscopy

EPR Electron Paramagnetic Resonance

FPLC Fast protein liquid chromatography

GAB General actin buffer

GSK3 $\beta \quad$ Glycogen Synthase Kinase-3 $\beta$

HOHAHA Homonuclear Hartmann Hahn

HSQC Heteronuclear Single Quantum Correlation

IPTG Isopropyl $\beta$-D-1-thiogalactopyranoside

ITC Isothermal Titration Calorimetry

LatB Latrunculin B 
MAPs Microtubule-associated proteins

MAPK Mitogen-activated Protein Kinase

MARK Microtubule Affinity Regulating Kinase

MBD Microtubule-binding domain

MTs Microtubules

MTSL 1-oxy-2,2,5,5-tetramethyl-d-pyrroline-3-methyl-methanethiosulfonate

NBD 7-chloro-4-nitrobenzeno-2-oxa-1, 3-diazole

NFTs Neurofibrillary Tangles

NMR Nuclear Magnetic Resonance

NOESY Nuclear Oberhausen Effect Spectroscopy

PB Polymerization buffer

PCS Pseudo Contact Shift

PHF Paired Helical Filaments

PKA Protein Kinase A

PKC Protein Kinase C

RDC Residual Dipolar Couplings

SB Separation buffer

ssNMR Solid-state Nuclear Magnetic Resonance

STD Saturation Transfer Difference

T 34 Thymosin- $\beta 4$

TIRF Total Internal Reflection Fluorescence

TOCSY Total Correlation Spectroscopy

TROSY Total Relaxation Optimization Spectroscopy

UPL Upper distance limits

WASP Wiskott - Aldrich syndrome Protein

WH2 WASP Homology 2 


\section{Introduction}

\subsection{Alzheimer's disease}

Alzheimer's disease (AD) is characterized as progressive brain disorder that slowly destroys memory and thinking skills and thereby suppresses the ability to carry out the simplest tasks. Around 46.8 million people worldwide were living with dementia in 2015. This number will almost double every 20 years, reaching 74.7 million in 2030 and 131.5 million in 2050. These new estimates are 12-13\% higher than those made for the World Alzheimer Report 2009 (Prince, Wimo et al. 2015). For these reasons $\mathrm{AD}$ has become one of the most important health and socioeconomic problems.

a

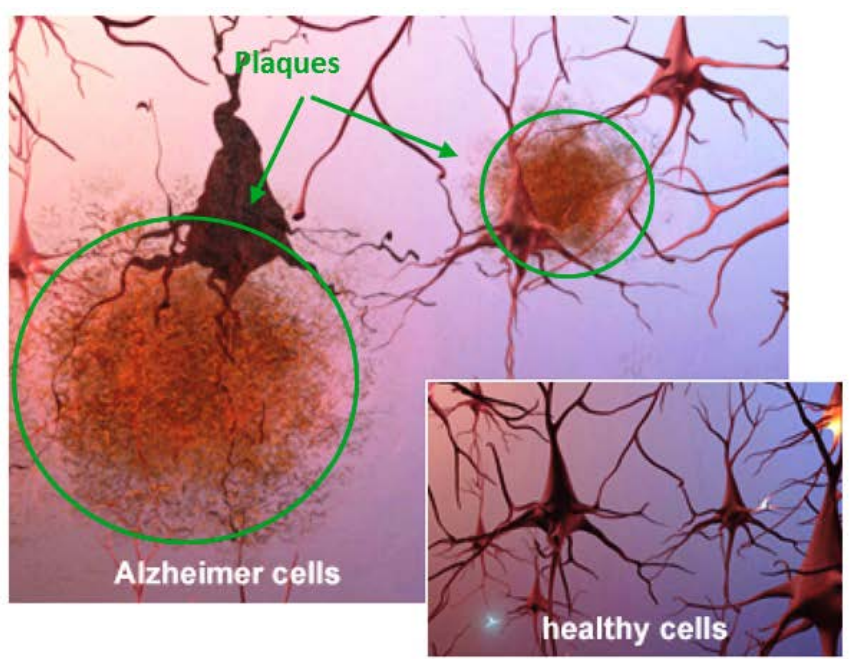

b

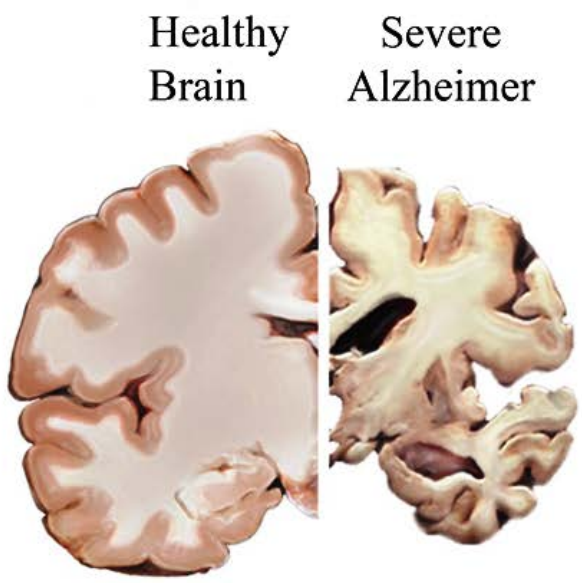

Figure 1.1. Progressive degeneration from neuronal to cerebral cortex.

(a) Extracellular neuritic plaques (green circles) are a pathological hallmark in AD. These plaques are composed of the insoluble aggregates of amyloid $\beta(\mathrm{A} \beta)$, a small proteolytic fragment from the amyloid precursor protein. (b) Progressive neuronal death is correlated with a loss of cerebral mass in the human brain (Image courtesy of National Institute of Health, modified to use here).

$\mathrm{AD}$ causes a large loss in brain weight and volume (Figure 1.1b) and affects some brain regions and neuronal populations more than others (GomezIsla, Price et al. 1996). Although AD causes 
loss of neurons in specific brain regions the overall loss of brain mass appears to be due to the shrinkage and loss of neuronal processes.

Since the time of Dr. Alois Alzheimer, neuropathologists have identified amyloid plaques and Neurofibrillary Tangles (NFTs) in the autopsied brains of people with AD, suggesting that these pathologies cause the disease (Ramirez-Bermudez 2012). Amyloid plaques are insoluble extracellular aggregates of $A \beta$ around neurons (Figure 1.1a) whereas NFTs are composed largely of paired helical filaments of hyper-phosphorylated Tau proteins, with both of them promoting neuronal and synaptic loss (Anand, Gill et al. 2014). NFTs have been found not only in AD but also in other Tauopathies including frontotemporal dementia, progressive supranuclear palsy, and corticobasal degeneration (Iqbal, Alonso et al. 2005).

\subsection{Tau pathology in Alzheimer's disease}

$\mathrm{AD}$ is related to the dysfunction of multiple proteins, which can adopt pathogenic conformations and accumulate in the brain. As mentioned before $\mathrm{AD}$ is associated not only with the abnormal accumulation of $A \beta$ plaques, but also with that of NFTs (Figure 1.2). NFTs are intracellular aggregates and are made up primarily of aggregated Tau bearing abnormal posttranslational modifications, including hyper-phosphorylation and acetylation (Min, Cho et al. 2010, Cohen, Guo et al. 2011).

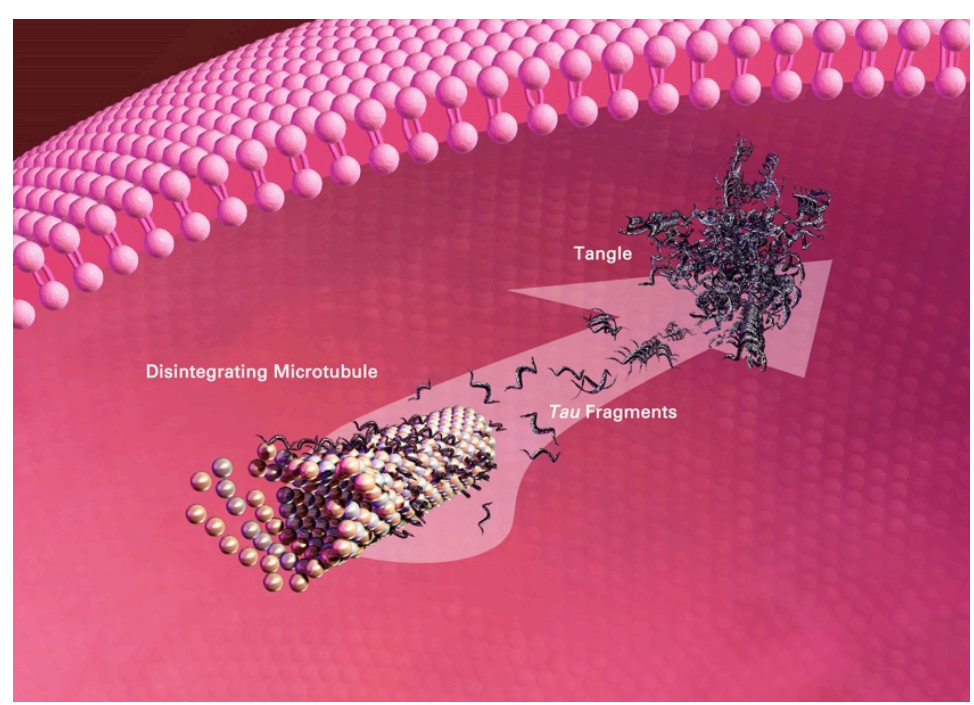

Figure 1.2. Microtubule disassembly and formation of neurofibrillary tangles.

Neurofibrillary tangles are aggregates of hyper-phosphorylated Tau. Tau with AD-like phosphorylation has an imparied ability to stabilize microtubules, destabilizing the cytoskeletal network and disrupting microtubule-supported cellular transport. (Image courtesy of the National Institute of Health) 
Tau is classically considered as a neuronal microtubule-associated protein that stabilizes microtubules (MTs) and supports the outgrowth of axons (Weingarten, Lockwood et al. 1975, Drubin and Kirschner 1986). It has being suggested that Tau-induced neurodegeneration is mediated by a loss-of-function mechanism due to hyper-phosphorylation and sequestration of Tau in solution (Zhang, Maiti et al. 2005). Nevertheless this idea have being broadly discussed and somehow remains as an open question because of the lack of evidences (Morris, Maeda et al. 2011) Tau may facilitate or enhance excitatory neurotransmission by regulating the distribution of synaptic activity-related signaling molecules (Morris, Maeda et al. 2011). However, abnormal modification drives to pathogenic conformations and Tau becomes enriched in dendritic spines where it can interfere with neurotransmission (Hoover, Reed et al. 2010). Entry of Tau into dendrites is correlated with a loss of dendritic spines and thus a decay of neuronal communication (Zempel, Thies et al. 2010). Furthermore, disruption of actin structures in growth cones perturbs the localization of Tau inside growth cones (Zmuda and Rivas 2000). It has also been found that Tau neurotoxicity correlates with alterations of actin organization in animal models of Alzheimer’s disease (Fulga, Elson-Schwab et al. 2007).

Therefore, inhibition of Tau abnormalities represents a promising therapeutic approach to AD and other tauopathies (Churcher 2006, Aisen, Cummings et al. 2012).

\subsection{Tau Protein}

Tau is an axonal protein discovered in the mid-1970s by studying factors necessary for microtubule formation (Weingarten, Lockwood et al. 1975). It is an intrinsically disordered protein, which is part of a group of proteins called Microtubule-associated proteins (MAPs) (Cassimeris and Spittle 2001). MAPs are heat resistant and not affected by acid treatment, retaining microtubule-binding even after harsh treatment (Cleveland, Hwo et al. 1977).

\subsubsection{Tau gene}

Tau is expressed in higher eukaryotes and found in both neuronal and non-neuronal cells, but predominantly in neurons (Loomis, Howard et al. 1990, Martin, Latypova et al. 2011). It is most abundant in neuronal axons (Lee, Goedert et al. 2001), but can also be found in neuronal somatodendritic compartments (Tashiro, Hasegawa et al. 1997) and in oligodendrocytes (Klein, Kramer et al. 2002). 
Human Tau is encoded in over $100 \mathrm{~kb}$ on the long arm of chromosome 17, specifically at position 17b21 and contains 16 exons (Figure 1.3, top panel). Exons 1, 4, 5, 7, 9, 11,12 and 13 are constitutive exons while exons 2, 3 and 10 are alternatively spliced (Sergeant, Delacourte et al. 2005). Exons 0, which is part of the promoter, and 14 are transcribed but no translated. Exons 6 and 8 are not transcribed in human brain while 4a is only expressed in the peripheral nervous system.

Alternative splicing of the N-terminal region and the repeat domain generates six different isoforms in the central nervous system (Sergeant, Delacourte et al. 2005). Tau isoforms are commonly named according to the number of microtubule-binding repeat sequences (termed $\mathrm{R}$ ) and whether N-terminal exons are included (termed N) (Figure 1.3). For example, 0N/3R correspond to hTau23 (see Figure 1.3, button panel) and 2N/3R for hTau39.

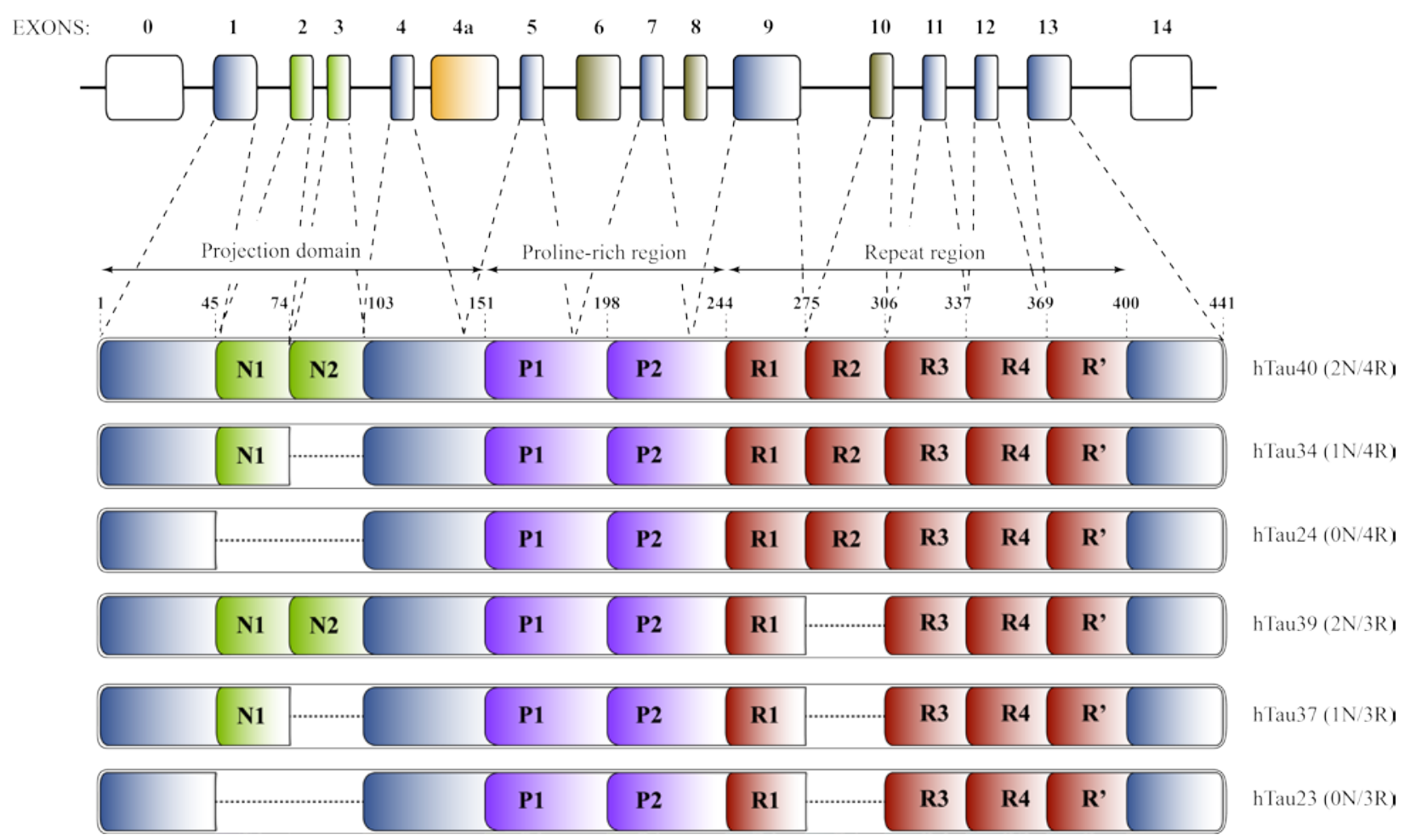

Figure 1.3. Schematic representation of the human Tau gene and the six Tau isoforms expressed in the adult central nervous system.

The Tau gene comprises 16 exons (upper panel) and eight of them are constitutive. Exons 2, 3 and 10 can be alternatively spliced giving rise to the six different Tau isoforms. Insert N2 can appear together with insert N1 in the projection domain (isoforms hTau40 and hTau39) but never alone. Meanwhile insert N1 can appear alone in isoforms hTau34 and hTau37. Exons 2 and 3 are transcribed but not translated in isoforms hTau24 and hTau23, while exon 10 is not translated in isoforms hTau39, hTau37 and hTau23. 
Tau can be subdivided into four regions: an N-terminal projection region, a proline-rich domain, a microtubule-binding domain (MBD), and a C-terminal region (Mandelkow et al., 1996).

\subsubsection{Tau phosphorylation}

Phosphorylation of Tau regulates its binding to microtubules, regulates their assembly and is associated with Tau aggregation in AD (Martin, Latypova et al. 2013). Phosphorylation of Tau around the MBD may neutralize the positive charge (Jho et al., 2010) and alter the conformation of the MBD of Tau (Fischer et al., 2009), detaching Tau from microtubules.

Depending not only on the position but also on the number of phosphorylated residues, the effect on MT polymerization and MT binding is different. For instance, phosphorylation of residues such as Ser214 (Illenberger, Zheng-Fischhofer et al. 1998), Thr231 (Lu, Wulf et al. 1999, Cho and Johnson 2004, Sottejeau, Bretteville et al. 2015), and Ser262 (Biernat, Gustke et al. 1993) decreases the MT/Tau-affinity and inhibits Tau's ability to promote MT assembly. On the other hand, phosphorylation of Ser202, Ser235, or Ser396 has almost no effect on MT binding and abolishes the tau-induced microtubule self-assemble (Utton, Vandecandelaere et al. 1997, Amniai, Barbier et al. 2009).

Tau has a total of 85 residues (5 tyrosines, 45 serines, 35 threonines,) (Hanger, Anderton et al. 2009) that can be phosphorylated either by non-proline directed kinases (PKA, PKC, MARK, CAMKII) (Correas, Diaz-Nido et al. 1992) or by proline-directed kinases (GSK3 $\beta$, cdk5, MAPK, p38) (Hanger, Hughes et al. 1992, Lucas, Hernandez et al. 2001). Ser262 phosphorylation, which is located within the conserved KXGS motif of the first MBD of Tau, has been suggested to be at the top of the phosphorylation cascade preceding phosphorylation of Ser202, Thr205, Ser396, and Ser404, and leading to Tau hyper-phosphorylation (Matenia and Mandelkow 2009, Bertrand, Plouffe et al. 2010). Mutation of Ser262 to alanine rescues the MT assembly properties of Tau, which are lost when wild-type Tau is phosphorylated by brain extracts. Moreover, alanine mutation on Ser262 and Ser356 residues in cell and animal models rescues the toxic effects of this type of phosphorylation in neuronal cells, but inhibits the outgrowth of neuronal processes highlighting the importance of these phosphorylation sites for neuronal differentiation (Yu, Polepalli et al. 2012).

Tau can be phosphorylated in vitro at KXGS motifs by several kinases, although different kinases phosphorylate the different sites to different extents (Hanger, Anderton et al. 2009). 
Efficient phosphorylation of the KXGS motifs is facilitated by the Microtubule AffinityRegulating Kinases (MARKs) and other members of the family of adenosine monophosphateactivated protein kinases (Yoshida and Goedert 2012). It has been shown by NMR that MARK2 binds to the N-terminal tail of Tau and selectively phosphorylates three major and five minor serine residues in the repeat domain and C-terminal tail. Structural changes induced by phosphorylation of Tau by MARK2 are highly localized in the proximity of the phosphorylation site and do not affect the global conformation of Tau (Schwalbe, Biernat et al. 2013).

\subsubsection{Tau structure}

Tau belongs to the class of intrinsically disordered proteins and exchanges between different conformations in solution (Jeganathan, von Bergen et al. 2006, Mylonas, Hascher et al. 2008). However, the polypeptide chain is not fully extended but adopts a paperclip shape, where the Nand C-terminal domains approach each other as well as the repeat domain (Jeganathan, Hascher et al. 2008). To obtain further insight into the dynamic structure of Tau in solution, NMR spectroscopy was used (Mukrasch, Bibow et al. 2009). Following a divide and conquer strategy, different Tau constructs were used to overcome the size limitation for NMR resonance assignment of intrinsically disordered proteins (Mukrasch, Bibow et al. 2009, Narayanan, Durr et al. 2010, Fauquant, Redeker et al. 2011). Based on paramagnetic resonance enhancement (PRE), an ensemble description of monomeric Tau in solution was then determined (Mukrasch, Bibow et al. 2009). The analysis showed that Tau is highly dynamic in solution, but has a distinct domain character with an intricate network of transient intramolecular contacts that are important for pathogenic aggregation (Mukrasch, Bibow et al. 2009).

Upon binding to interaction partners such as MTs, distinct regions within the Tau protein can fold into stable structure. Early studies have already shown that the repeat domain and the neighboring proline-rich regions contribute strongly to MT binding (Butner and Kirschner 1991). Moreover, regions outside of the MBD may influence the spacing between MTs (Chen, Kanai et al. 1992). In addition, a variety of binding models of the Tau/MT complex have been proposed (Al-Bassam, Ozer et al. 2002, Kar, Fan et al. 2003, Makrides, Massie et al. 2004, Santarella, Skiniotis et al. 2004, Gigant, Landrieu et al. 2014). A Tau fragment from S208 to S324 (F4) adopts a U-turn like conformation when binds to a single tubulin dimer in the presence of stathmin. However it gets an extended conformation when stathmin is not present, promoting straight protofilaments in microtubules (Gigant, Landrieu et al. 2014). More recently, in our lab, 
Kadavath et al. found that Tau binds microtubules through short sequence motif at the interface between $\alpha$ - $\beta$-tubulin heterodimers (Kadavath, Hofele et al. 2015). Upon binding to microtubules, the MT-binding motifs of Tau fold into a stable hairpin-like structure (Kadavath, Jaremko et al. 2015). This hairpin-like model is consistent with recent FRET-based studies were authors suggest Tau experiences local changes upon binding to tubulin heterodimers supported by its intrinsic flexibility (Melo, Coraor et al. 2016). Nevertheless, no evidences were found about the U-turn like structure reported by Giant, Landrieu et al.

Barre and Elizer showed by using NMR that short segments of the repeat region of Tau, adopts a stable $\alpha$-helix conformation in the presence of anionic micelles, suggesting a structural reorganization of the protein in membrane-like environments (Barre and Eliezer 2006). In addition, several labs determined that a short stretch of residues corresponding closely to a previously identified Paired Helical Filaments (PHF) nucleation site (von Bergen, Friedhoff et al. 2000) exhibits a marked preference for $\beta$-strand structure (Kunze, Barre et al. 2012, Barre and Eliezer 2013, Huvent, Kamah et al. 2014), supporting the importance of $\beta$-sheet formation in the Tau aggregation process. Protease digestion and mass spectrometry experiments revealed that the core of PHF comprises Tau segments from the first repeat to the C-terminus separated by dynamic loops, which are accessible to proteolysis (von Bergen, Barghorn et al. 2006). Other experiments indicated that cross- $\beta$ structures are present on PHF with a preferential orientation to the fiber axis (Giannetti, Lindwall et al. 2000, von Bergen, Barghorn et al. 2001, Berriman, Serpell et al. 2003). This finding was further supported by electron paramagnetic resonance (EPR) and solid-state NMR (ssNMR) experiments where these $\beta$-strands were found to be parallel oriented (Margittai and Langen 2004, Margittai and Langen 2006, Daebel, Chinnathambi et al. 2012). In addition the ssNMR study indicated that a well-defined rigid core from V306 to S324 in K19 composed of three $\beta$-strands (Daebel, Chinnathambi et al. 2012).

\subsubsection{Tau functions}

The main known function of Tau is to stabilize MTs, modulating the transport of vesicles and organelles along MTs (Witman, Cleveland et al. 1976, Spittaels, Van den Haute et al. 2000, Dixit, Ross et al. 2008, Nam and Epureanu 2017, Stern, Lessard et al. 2017). Nevertheless Tau is also a multifunctional protein having numerous binding partners (Uversky 2015), including signaling molecules, cytoskeletal elements and lipids. Tau can bind to cytoskeletal proteins and 
regulate signaling pathways (Godoy, Rios et al. 2014) . Tau binding also activates or inhibits several enzymes (Morris, Maeda et al. 2011).

Furthermore, Tau induces changes in the organization and stability of actin filaments in neurons, which in turn contribute to Alzheimer disease-like neurodegeneration in Drosophila and mouse model systems (Fulga, Elson-Schwab et al. 2007). Tau-induced actin-rich rods were found to induce neurodegeneration in Drosophila neurons (Fulga, Elson-Schwab et al. 2007). In the same study, Tau-induced neurotoxicity was found to be associated with a panneural increase in F-actin levels, while G-actin levels remained unchanged.

Subpopulations of Tau interact with microtubules and actin filaments in various cell types (Henriquez, Cross et al. 1995). This interaction is mediated primarily by the MBD (Farias, Munoz et al. 2002, Yu and Rasenick 2006) and is assisted by the adjacent proline-rich domain (He, Wang et al. 2009). Tau also cross-links MTs with actin filaments (F-actin) via its MBD, promoting in vitro co-organization and coupled growth of both networks (Elie et al., 2015;Farias et al., 2002).

\subsection{Actin protein}

Seven and halve decades ago, in the early 1940s, actin was first isolated from skeletal muscle. Scientists discovered then that actin is an essential protein for muscle contractility and can exist in both fibrous (F-) and globular (G-) states. It was not until 1970 when actin was also found in non-muscle cells and organisms - first in Acanthamoeba (Pollard, Shelton et al. 1970) and then in various mammalian non-muscle cells (Lazarides and Weber 1974).

Because actin polymerizes or aggregates under crystallization conditions, the first X-ray structure of G-actin was solved only in 1990 (Kabsch, Mannherz et al. 1990), in complex with the actin binding protein DNase I which blocks actin polymerization, leading to the first atomic model of F-Actin (Holmes, Popp et al. 1990).

\subsubsection{G-actin}

Actin is a globular protein of around $43 \mathrm{kDa}$ and is the major component of the cytoskeleton in eukaryotic cells (Cooper 2000). It is involved in cell motility, cargo transport, muscle contraction, and cytoskeletal integrity (Dominguez and Holmes 2011). Actins comprise a highly conserved family of proteins that fall into three broad classes: alpha, beta, and gamma isoforms 
(Herman 1993). These isoforms differ from one another in only the first 4-5 amino acids at their N-terminus (Vandekerckhove and Weber 1978). Alpha actins are found in muscle tissues, which are an important constituent of the contractile apparatus while beta and gamma actins are present in most cell types as components of the cytoskeleton where they mediate cell motility. Nevertheless recent studies with mouse models revealed the presence of alpha-actin in motoneurons (Moradi, Sivadasan et al. 2017)..

G-actin is globular has 375 amino acids with dimensions around $67 \times 40 \times 37 \AA$, contains a bound nucleotide and can exist in the ATP, ADP-Pi or ADP form (Bugyi and Carlier 2010).

a

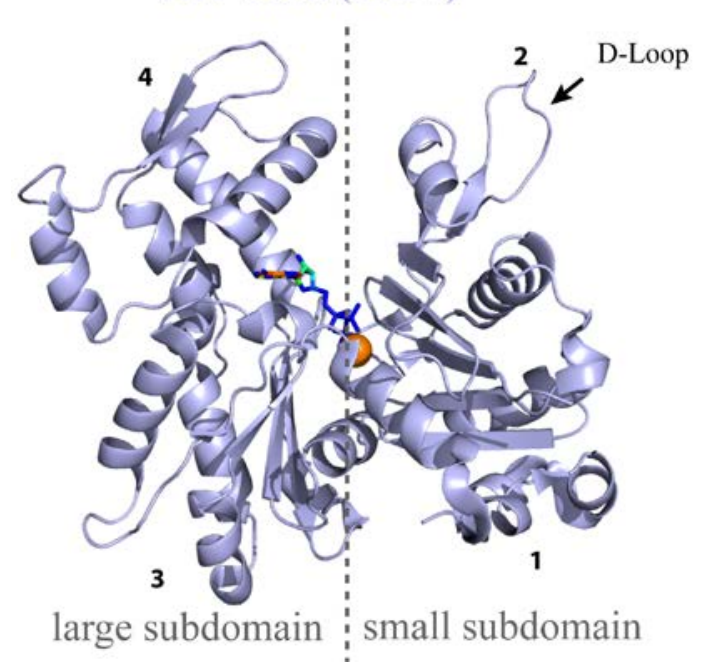

b

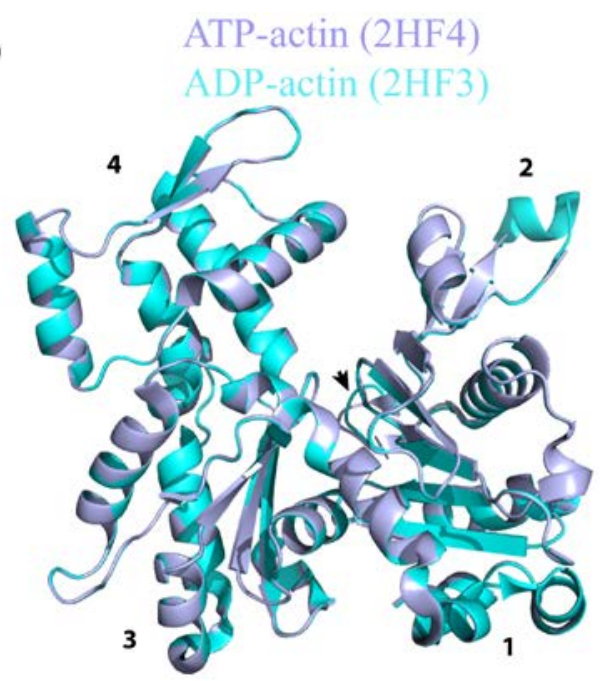

Figure 1.4. Comparison between ATP- and ADP-bound actin crystal structures.

(a) Crystal structure of ATP-bound actin where the different subdomains are labeled from 1 to 4 . ATP molecule appears at the nucleotide-binding pocket with a bluecolor and having a divalent cation $\left(\mathrm{Mg}^{2+}\right)$ bound to it (dark yellow). (b) Superposition of ATP-bound (light blue) and ADP bound (cyan) actin. Nucleotides are not shown for clarity. Structures are nearly the same except for the loop nearby the nucleotide binding (arrowhead) and the small $\alpha$-helix in subdomain 2 in case of ADP-actin. The loop or the short helix on subdomain 2 was built on Pymol because it is not resolved in these crystal structures. PDB codes are indicated (Rould, Wan et al. 2006).

G-actin consists of two major domains: the small and the large domains, which are again divided into subdomains 1, 2, 3 and 4 (Figure 1.4). A nucleotide (ATP or ADP) is positioned deep inside the cleft between subdomain 2 and 4 . This region is also a binding site for many actin-binding proteins (Dominguez 2004) and small toxins like latrunculins (Yarmola, Somasundaram et al. 2000). The outer part of subdomain 2 (D-loop; residues 40-51), is disordered in most X-ray structures of actin published to date, but has been also observed in a $\alpha$-helix, $\beta$-turn, and various 
loop conformations especially in presence of ADP (Figure 1.4b). Both the N- and C-termini of actin are located in subdomain 1, albeit on opposite faces of the molecule. Similar to the D-loop, $\mathrm{N}$ - and C-termini are flexible and are often disordered in X-ray structures.

G-actin can exist in different conformations, depending on the status of the actin-bound ATP, the nature of the divalent cation $\left(\mathrm{Ca}^{2+}\right.$ or $\left.\mathrm{Mg}^{2+}\right)$ at the high-affinity site or at additional sites, and the degree of oligomerization (Moraczewska, Wawro et al. 1999, Schuler 2001). Assuming an invariant, diffusion-limited association rate constant of $6 * 10^{6} \mathrm{M}^{-1} \mathrm{~s}^{-1}, \mathrm{Ca}^{2+}$-actin binds ATP several times stronger than $\mathrm{Mg}^{2+}$-actin (Frieden 1982, Kinosian, Selden et al. 1993)

The hydrophobic pocket of actin is considered to be one of the most important binding sites and it is recognized by most of the actin binding partners (Bobkov, Muhlrad et al. 2002, Dominguez 2004, Hertzog, van Heijenoort et al. 2004, Chereau, Kerff et al. 2005, Didry, Cantrelle et al. 2012). This pocket is comprised between subdomains 1 and 3, sometimes referred as the barbed end when actin is assembled into filaments.

\subsubsection{F-actin}

Actin Filaments are found in most eukaryotic cells as constituents of the cytoskeleton. They play a central role in various types of motility (including muscle contraction) and transport processes. Holmes and co-workers were the first to propose an atomic model of F-actin, i.e., the HolmesLorenz model (Holmes, Popp et al. 1990, Lorenz, Popp et al. 1993).

Later, Schutt and colleagues have constructed an alternative atomic model of F-actin derived from the structural analysis of bovine profilin $\beta$-actin co-crystals (Schutt, Rozycki et al. 1995, Schutt, Kreatsoulas et al. 1997). Their model is slightly different from the Holmes-Lorenz model, although it is corroborated by the same structural constraints as were used to build and refine the former model.

More recently, thanks to advances in cryo-electron microscopy (Cryo-EM) better resolved structural models of F-actin became available (Fujii, Iwane et al. 2010, Galkin, Orlova et al. 2011). Recently a Cryo-EM-based three-dimensional structure of F-actin at a resolution of $3.7 \AA$ in complex with tropomyosin was reported (von der Ecken, Muller et al. 2015).

Within actin filament the distance between subunits on adjacent strands is $27.3 \AA$ with a rotation of $166.15^{\circ}$ around the axis (Egelman, Francis et al. 1982, Fujii, Iwane et al. 2010). Filaments are 
flexible, and have a helical repeat every $37 \mathrm{~nm}$, ranges from 5-9 nm in diameter with 13 actin subunits between each crossover point (Egelman 1985, Egelman 2003).

\subsubsection{F-actin assembly}

The actin filament is functionally and structurally asymmetric, which in vitro is reflected in a difference in the rate of addition of actin monomers at both ends. It is composed of numerous individual actin subunits bound together to form a polar double-stranded structure (Oda, Iwasa et al. 2009). The polymerization process occurs in three different sequential phases (Figure 1.5). The first phase is marked by a latency period in which G-actin aggregates into short, unstable oligomers that are more likely to rapidly dissociate to monomers than to assemble. Once the oligomers reach a certain size (three to four monomers) it is used as a seed from which an elongation process (second phase) takes place. In this phase actin monomers are added to both () and (+) ends where actin assembly is more likely than disassembly. As long as the actin filaments grow, the concentration of G-actin monomers decreases until it is in equilibrium with the filament. This third phase is called "steady state" or "treadmilling" because G-actin monomers exchange with subunits at the filament ends but there is no net change in the total mass of filaments. At this stage ATP-actin is more efficiently incorporated at the $(+)$ end than to the (-) end therefore the filament elongation takes place over the $(+)$ end.

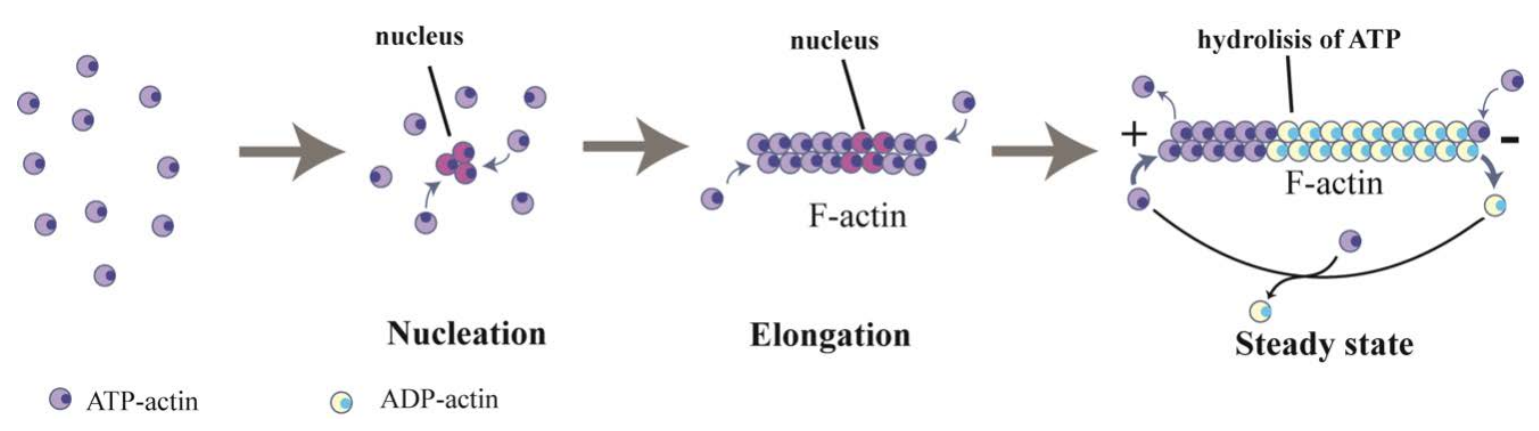

Figure 1.5. The three different phases of actin polymerization.

During the initial nucleation phase, ATP-actin monomers (pink) slowly form stable trimeric or tetrameric complexes of actin (purple) called nucleus. These nuclei are more rapidly elongated in the second phase by addition of subunits to both ends of the filament. In the steady state, the filament dynamics enter a state of equilibrium where monomer disassembly from the $(-)$ end and polymerization at the $(+)$ end is balanced and maintained by a critical concentration of monomers in the cytosol. After their incorporation into a filament, subunits slowly hydrolyze ATP and become stable ADP-F-actin (yellow). Blue circles highlight ATP-binding clefts 
In vivo elongation at the barbed end is 10 -to 20 - fold faster than at the slow polymerizing pointed end. Therefore the polymerization occurs with higher efficiency at the barbed end (Bugyi and Carlier 2010). Thus, during the initial phase of polymerization in vitro, in the presence of excess ATP, ATP-actin is rapidly and preferentially incorporated into filaments at their barbed end creating a ‘cap’ rich in ATP-subunits (Pollard, Blanchoin et al. 2000).

A minimum concentration of monomers in the cytosol is needed for the actin polymerization to occur; this is known as critical concentration (Cc). The Cc depends on the isoform of actin, the nucleotide and divalent cation bound in the nucleotide cleft, solvent conditions ( $\mathrm{pH}$, ionic strength, temperature, etc.), and the presence of other actin binding proteins or factors. When the concentration of free subunits exceeds the Cc, filament elongation occurs spontaneously (Carlier and Pantaloni 1997). For the (+) end the Cc is assumed to be $\sim 0.1 \mu \mathrm{M}$ whereas in the (-) end it is $\sim 0.8 \mu \mathrm{M}$ (Lodish H 2000). At the steady state, which is achieved when the rate of filament polymerization is equally balanced by filament disassembly, the free subunit concentration is higher than the $\mathrm{Cc}_{\mathrm{c}}$ at the $(+)$ end and lower than the $\mathrm{Cc}_{\mathrm{c}}$ at the (-) end. As a result subunits are added to the $(+)$ end and dissociates from the $(-)$.

\subsubsection{Actin partners}

The dynamic behavior and the organization of the actin cytoskeleton are regulated by numerous actin-binding proteins, responsible for nucleating, binding to G- and F-actin, severing F-actin, capping its end, and bundling filaments (Sokolov, Spooner et al. 1999, Galkin, Orlova et al. 2011)

\subsubsection{G-actin partners}

Different G-actin binding drugs and proteins have been identified (dos Remedios, Chhabra et al. 2003, Dominguez 2004). There are at least four different families of proteins that bind primarily to actin monomers. G-actin binding proteins are very important in nature because they are part of the machinery needed for modulating the polymerization rate by controlling the amount of Gactin in the cytosol. Other proteins including profilin mediate phosphorylation of ADP-actin (Nishida 1985, Vinson, De la Cruz et al. 1998) and therefore play an important role in the treadmilling process.

The pancreatic secretory protein DNase-I binds to most G-actin isoforms with a dissociation constant of $5^{*} 10^{8} \mathrm{M}^{-1}$ (Mannherz, Leigh et al. 1975, Mannherz, Goody et al. 1980). It binds to 
the D-loop and inhibits nucleotide binding, because of its proximity to the nucleotide binding site as well as the conformational changes in actin upon binding (Kolli, Wojes et al. 2008).

The best-studied sequestering G-actin binding proteins belong to the Thymosin family. These proteins act by clamping ATP-actin top to bottom capping the actin monomers at both barbed and pointed ends and preventing incorporation into filaments (Hertzog et al., 2004; Irobi et al., 2004). More recently the structure of Thymosin- $\beta 4$ (T $\beta 4$ ), the major variant of $\beta$-thymosins, in complex with actin was determined by NMR (Didry, Cantrelle et al. 2012). T $\beta 4$ is known to be unstructured in solution but adopts $\alpha$-helical conformation upon binding to actin (Zarbock, Oschkinat et al. 1990, Hertzog, van Heijenoort et al. 2004, Didry, Cantrelle et al. 2012).

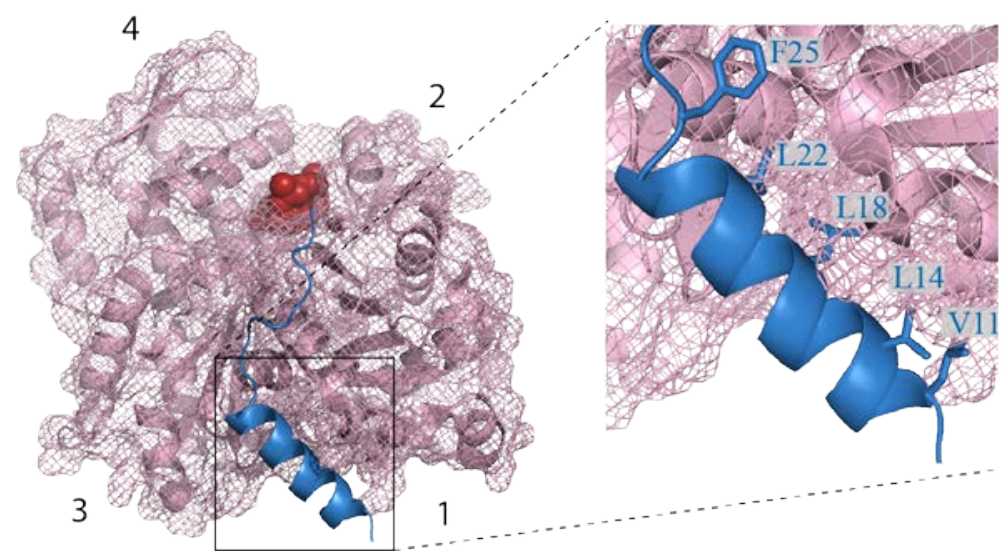

Figure 1.6. Crystal structure of actin in complex with ciboulot.

Latrunculin B is represented with red spheres and is bound to the nucleotide-binding pocket of the actin molecule (pink). Hydrophobic residues from ciboulot involved in binding are highlighted (right panel) (PDB id 1SQK (Hertzog, van Heijenoort et al. 2004)).

The actin-binding motif of $\beta$-thymosins are also found in various modular proteins, where it is called WH2 (WASP Homology 2) domain, because it was first recognized in proteins of the WASP (Wiskott-Aldrich Syndrome Protein) family. Some of these proteins consist of two or more $\beta$-thymosin repeats. ciboulot (Cib) is a protein from Drosophila, which has three different domains (D1, D2 and D3) (Boquet, Boujemaa et al. 2000). In spite of its sequence similarity with $\beta$-thymosin Cib displays a profilin-like function promoting barbed-end actin assembly while inhibiting pointed-end growth (Boquet, Boujemaa et al. 2000, Hertzog, Yarmola et al. 2002). It has been shown that the first domain of Cib (D1) interacts with actin and possesses the biochemical and motile activities of the full-length protein (Hertzog, van Heijenoort et al. 2004). Despite the fact that no ADP-actin binding activity has been reported so far, Cib binds with a high affinity to ATP-actin (Kd=3 $\mu \mathrm{M})$ (Hertzog, Yarmola et al. 2002) adopting an $\alpha$-helical 
conformation upon binding (Figure 1.6). This interaction is mainly hydrophobic (Hertzog, van Heijenoort et al. 2004) and apparently all the hydrophobic residues on the $\mathrm{N}$-terminus are contributing to the interaction.

The binding site of Cib on actin surface is typically referred as hydrophobic pocket of actin (Dominguez 2004) and it forms the binding site for several other proteins including gelsolin, cofilin, profilin. All these proteins adopt an amphipathic $\alpha$-helical conformation upon binding to actin (Dominguez 2004).

In addition to the identified G-actin-binding proteins, small molecules can also bind to actin. Despite the fact that most of them cannot be used for pharmacological treatments due to the inability to enter the cell, they are still widely used in research. Kabiramide, latrunculin and swinholide are known to bind to monomeric actin; however, the binding site and binding mode are different.

Swinholide A, a molecule isolated from marine sponges, is a 44-carbon ring dimeric dilactone macrolide with a 2-fold axis of symmetry (Figure 1.7a). This molecule sequester actin dimers in vitro in both polymerizing and non-polymerizing buffers in a binding stoichiometry of one swinholide molecule per actin dimer (De Marino, Festa et al. 2011). Swinholide A also severs Factin with high cooperativity. The crystal structure of this complex is shown in Figure 1.7b.

Kabiramide C, is a marine toxin of Trisoxazole family that binds to actin with a dissociation constant of $100 \mathrm{~nm}$ or less (Tanaka, Yan et al. 2003). The X-ray structure in complex with actin (1QZ5) is already available (Stricker, Falzone et al. 2010). The 3D structure reveals that the molecule interacts with subdomains 1 and 3 of actin (medium panel, Figure 1.7d). Despite the fact that molecules of the Trisoxazole family bind to the hydrophobic pocket of actin, they act similar to gelsolin (Galkin, Orlova et al. 2010) and profilin (Torres-Cruz, Rodriguez-Cruz et al. 2016) inhibiting the nucleotide exchange on actin. This effect may be related to the fact that the binding of kabiramide C may increase the rigidity of actin, which prevents the normal "open" and “close” of the nucleotide-binding pocket (Klenchin, Allingham et al. 2003). 
a

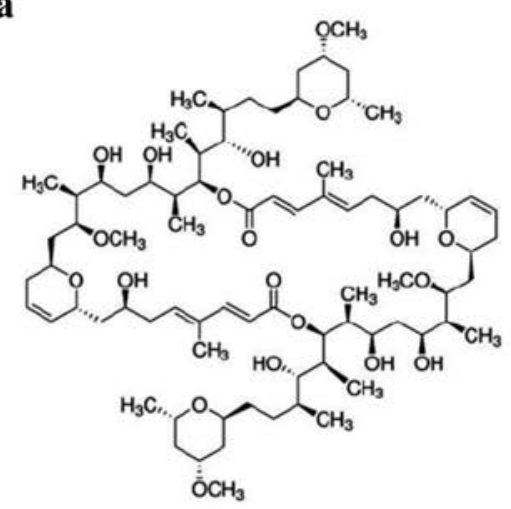

swinholide A

c

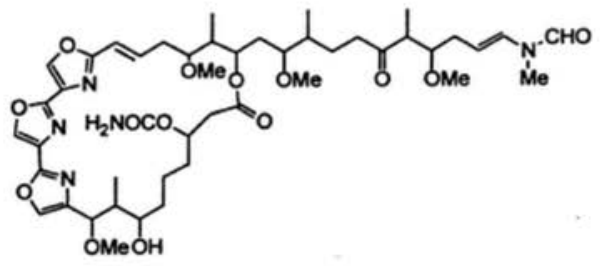

kabiramide $\mathrm{C}$

e<smiles>C/C=C(/C)CC/C=C(\C)CC/C(C)=C\C(=O)OC1CC(O)C(O)(C2CSC(=O)N2)C1</smiles>

latrunculin B

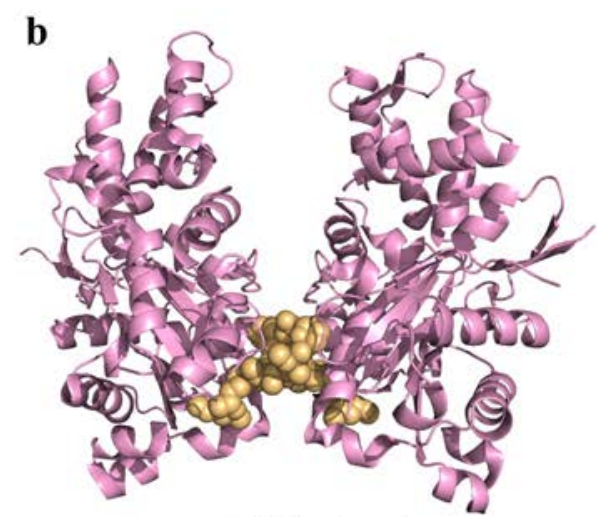

swinholide A-actin complex (1YXQ)

d

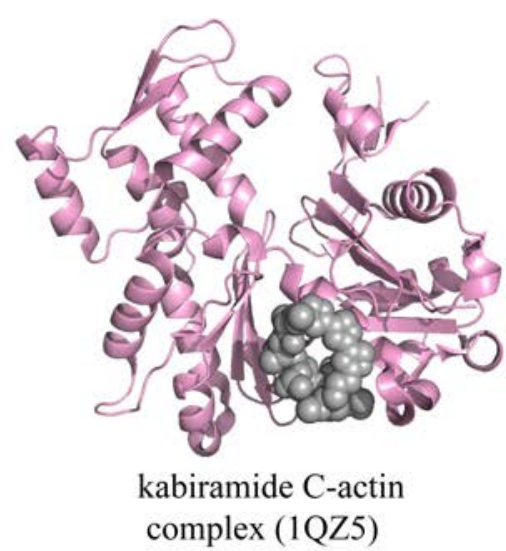

f

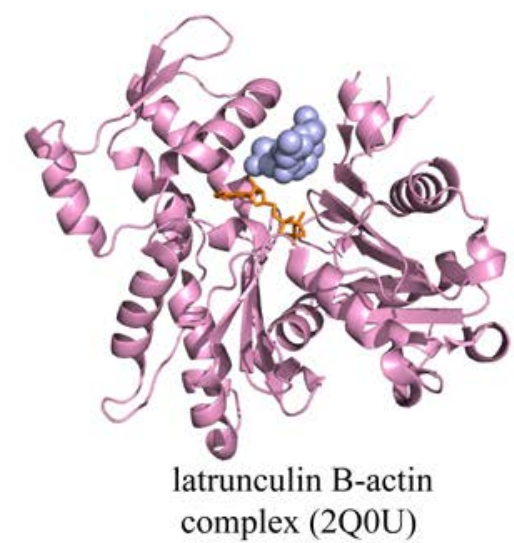

Figure 1.7. Small molecules and their complexes with G-actin.

(a) Chemical structure of swinholide A. (b) Cartoon representation of two actin molecules (pink) crosslinked by swinholide (yellow spheres). (c, e) Chemical structures of kabiramide C and latrunculin B respectively, with crystal structures in complex with actin presented in (d) and (f). Kabiramide C in grey binds on the hydrophobic pocket of actin, while latrunculin B (blue light spheres) blocks nucleotide (yellow) exchange on the nucleotide-binding cleft. 
Unlike kabiramide and swinholide, latrunculin molecules, isolated from the red sea sponge Negombatamagnifica, bind directly to the nucleotide exchange cleft between subdomains 2 and 4 (Guo, Shillcock et al. 2010, Dominguez and Holmes 2011) (bottom panel, Figure 1.7a). Two related compounds, latrunculin-A (LatA) and latrunculin-B (LatB), were shown to depolymerize actin structures both in vitro and in vivo (Kashman, Groweiss et al. 1980, Spector, Shochet et al. 1983). LatA binds to G-actin in a 1:1 molar complex with an equilibrium dissociation constant of $0.2 \mu \mathrm{M}$ (Pollard, Blanchoin et al. 2000). The molecule lowers the affinity of actin for T $\beta 4$ by 1 order of magnitude, but it does not affect the binding of profilin or DNase I (Yarmola, Somasundaram et al. 2000). LatA acts as a sequestering agent of actin, preventing F-actin assembly. A review from Allingham et al. describes the molecular mechanism by which different actin-targeting natural products function in detail (Allingham, Klenchin et al. 2006)

\subsubsection{F-actin partners}

F-actin-binding proteins can be divided into three different groups depending on their effect on actin filament dynamics and organization. The first group is the one comprising F-actin severing proteins. They have the ability to break actin filaments through a non-proteolytic mechanism. The second group consists of proteins, which are able to bind at either end of the filament, inhibiting further addition of monomers, so called capping proteins. The last group interacts with the lateral side of filaments and can stabilize, protect or reconnect the filaments.

Gelsolin is a $\mathrm{Ca}^{2+}$-regulated actin-binding protein that belongs to the family of actin-severing and actin-capping proteins, which includes adseverin, villin, CapG, advillin, and supervillin (Silacci, Mazzolai et al. 2004). Gelsolin is an $80-\mathrm{kDa}$ protein consisting of two tandem homologous halves (segments 1-3 and 4-6), each containing three repeats. Segment 1 (S1) binds G-actin in the absence of $\mathrm{Ca}^{2+}$, and the resulting complex is able to cap actin filaments. Segments 4-6 (S4S6) constitute a second, $\mathrm{Ca}^{2+}$-dependent, G-actin binding fragment that competes for the same actin-binding site as S1. S2 binds F-actin in a $\mathrm{Ca}^{2+}$-independent manner and is able to decorate actin filaments. Nucleation of F-actin can be achieved with S2-S6 but the F-actin-severing function is performed by S1-S3 (Burtnick, Koepf et al. 1997). 


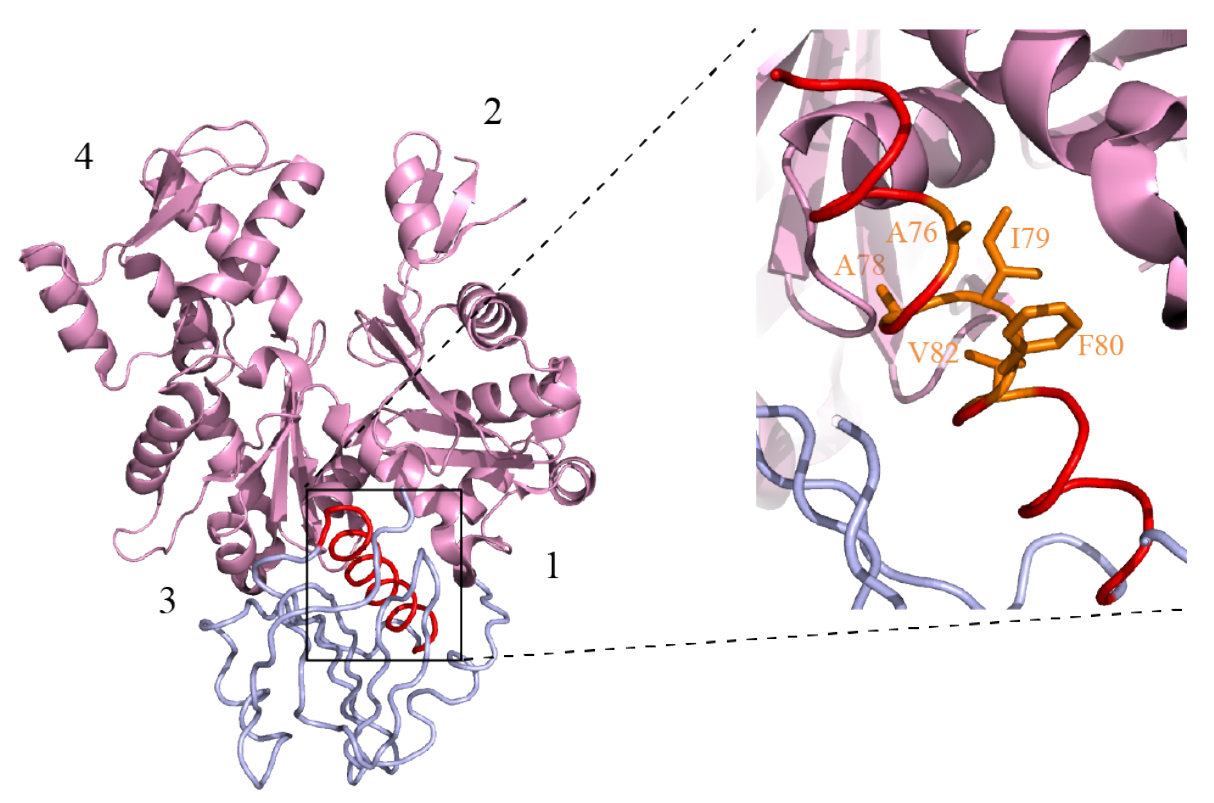

Figure 1.8. Crystal structure of G-actin in complex with gelsolin

Crystal structure of G-actin (pink) in complex with the subdomain S1 of gelsolin (blue) is shown (PDB id 1EQY (Mclaughlin, Gooch et al. 1993)). The $\alpha$-helix in close contact with actin is highlighted in red. In the right panel, hydrophobic residues from gelsolin, which are thought to be involved in binding, are highlighted in dark yellow.

In Figure 1.8 the crystal structure (PDB id 1EQY (Mclaughlin, Gooch et al. 1993)) of the complex of the S1 subdomain of gelsolin with G-actin is presented. Major protein-protein contacts involve an amphipathic helix formed by Ser70-Asn89 of gelsolin and an extended hydrophobic cleft located between subdomains 1 and 3 of actin. The $\alpha$-helix presents exposed hydrophobic side chains, which bind into the hydrophobic cleft of actin.

A second important and essential family of eukaryotic actin-binding proteins is formed by ADF/cofilins. ADF/cofilins have long been known to play a key role in actin filament dynamics and to have highly complex and interesting modes of regulation. ADF/cofilins are homologous actin-binding proteins, which bind to the side of the filament. Thus it has an important role in promoting actin filament turnover, which supports different forms of cell motility (Bernstein and Bamburg 2010, Bravo-Cordero, Magalhaes et al. 2013). Although cofilin is known to binds Factin, it can also accelerate spontaneous assembly of actin monomers (Carlier et al., 1997; Du and Frieden, 1998; Yeoh et al., 2002) 
cofilin concentration

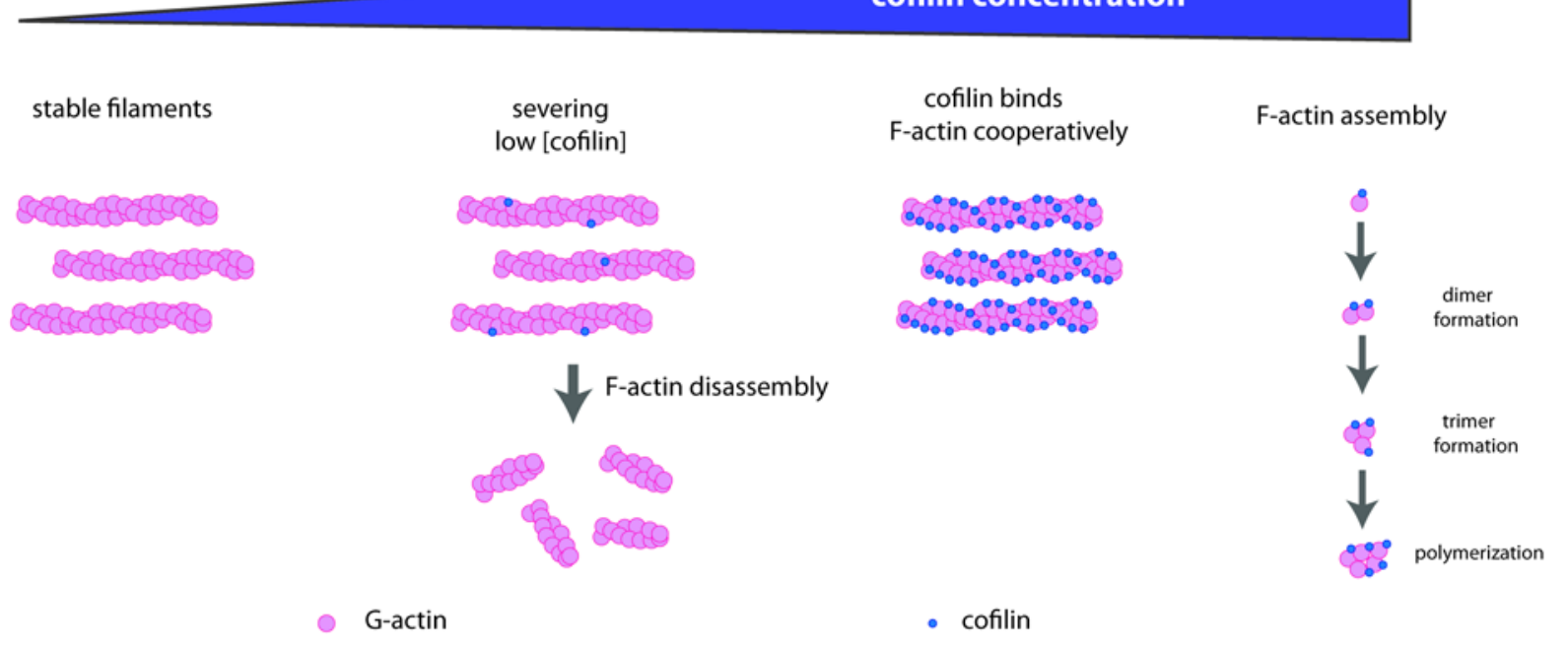

Figure 1.9. Concentration-dependent activity of cofilin.

Filaments remain stable in absence of cofilin. At low concentration, cofilin (blue circles) breaks the filaments up in shorter filaments. At higher concentrations of cofilin, cofilin binds to F-actin in a cooperative way promoting release of inorganic phosphate (Pi). At very high concentrations of cofilin, cofilin binds to G-actin and promotes filament assembly.

At low ratios with respect to actin subunits in F-actin (<1:100), cofilin severs F-actin forming more filament ends that can either nucleate filament growth or quickly depolymerize filaments, depending on the amount of available G-actin (Andrianantoandro and Pollard 2006). Whether cofilin binds the filaments depends on the release of inorganic phosphate (Pi) after actin ATP hydrolysis because Pi binds antagonistically with cofilin (Muhlrad, Pavlov et al. 2006). In addition, release of Pi is increased 10- fold by cofilin binding (Blanchoin and Pollard 1999). Cofilin's ability to depolymerize actin faster at higher $\mathrm{pH}$ is thought to result from the $\mathrm{pH}$ dependence of Pi release (Pavlov, Muhlrad et al. 2006), because the binding of Pi is stronger at low pH (6.5) than high pH (8.0) (Muhlrad, Pavlov et al. 2006). At higher cofilin-to-actin molar ratios, cofilin severs rapidly but transiently because it binds F-actin cooperatively and stabilizes F-actin in a twisted form as it saturates the severed pieces (Ni and Scheraga 1994) (Figure 1.9). 
a

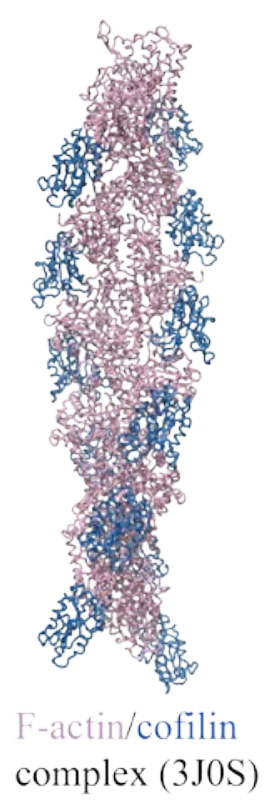

b

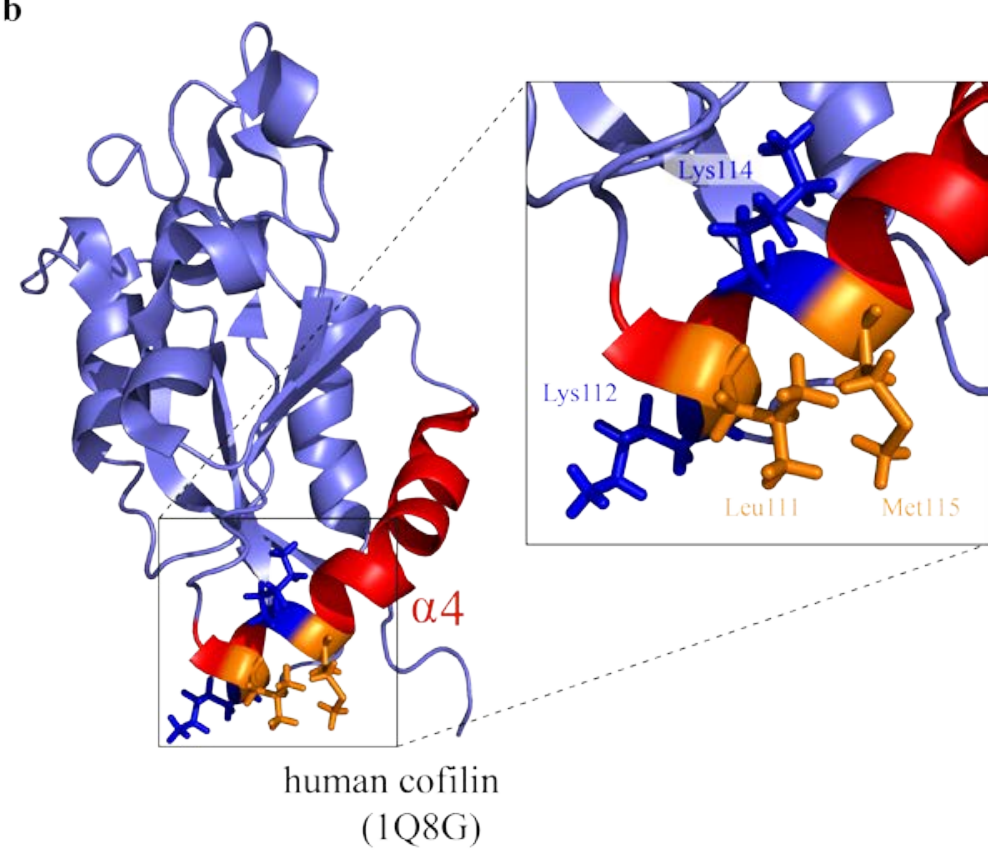

Figure 1.10. 3D structures of cofilin in complex with F-actin and in solution.

(a) Cofilin molecules (blue light) bind to the actin filament (pink) surface and decorate it (Hertzog, van Heijenoort et al. 2004). (b) Cartoon representation of human cofilin in solution. $\alpha$-helix 4, which is involved in binding to actin, is colored in red. Side chains of residues important for binding are highlighted (Bernstein and Bamburg 2010)

In 2003, Pope et al determined the 3D structure of human cofilin in solution (Pope, ZierlerGould et al. 2004). The N-terminal part of the long $\alpha 4$-helix of cofilin (Figure 1.10b) is involved in binding to the hydrophobic pocket of G-actin between subdomains 1 and 3 (Gronenborn 1983) (Pope, Zierler-Gould et al. 2004, Macchi, Rudd et al. 2016), and site-directed mutagenesis identified Lys112 and Lys114 in $\alpha 4$ as essential for G-actin binding (Hetenyi, Hegedus et al. 2016, Macchi, Rudd et al. 2016). Synchrotron protein foot printing identified residues in yeast cofilin corresponding to Met115 G-actin binding (Tang, Huang et al. 2016). According to the 3D structure of the cofilin/F-actin complex derived from Cryo-EM, the binding region on F-actin is the same (Galkin, Orlova et al. 2011). This is because the hydrophobic pocket of actin is exposed to the solvent on the filament surface (Dominguez 2004). Figure 1.10a shows how cofilin decorates the actin filament surface.

Another group of F-actin-binding proteins include Arp2/3, $\alpha$-actinin, vinculin, fascin, fimbrin, spectrin and filamin. They interconnect filaments in many different ways providing several functionalities within the cell. Arp2/3, for example, is a 7-subunit protein complex, which builds 
cross-linked actin networks facilitating cell movement. Arp2/3 builds these networks by binding to the sides of existing actin filaments and nucleates growth of new filaments from their sides.

Small molecules have also been reported to bind to and stabilize actin filaments. Some of these molecules are phalloidin (Cooper 1987), jasplakinolide (Bubb, Spector et al. 2000, Minamide, Striegl et al. 2000) and chondramide (Kunze, Jansen et al. 1995, Sasse, Kunze et al. 1998). While all three substances have the capacity to stabilize F-actin, phalloidin is not membrane permeable, while jaslplakinolide and chondramides readily enter cells (Sawitzky, Liebe et al. 1999). Therefore, phalloidin is used in cell biological research mainly for visualization of F-actin after fluorescence labeling of the compound in fixed tissues (Peterson and Mitchison 2002). In contrast, the commercially available jasplakinolide can be used for F-actin stabilization in living cells due to its membrane permeability (Matthews, Smith et al. 1997).

On the other hand cytochalasins, a group of fungal metabolites, binds to the $(+)$ end of F-actin and slow down the rate of filament formation by inhibiting the rate of elongation (Holzinger and Blaas 2016). Cytochalasin D (CD) binds to F-actin with a stoichiometry of one CD per actin filament with a very high affinity (Kd $2 \mathrm{nM}$ ) (Cooper 1987) and inhibit the interaction between cofilin and F-actin (Shoji, Ohashi et al. 2012). CD also binds to monomeric actin but with lower affinity (2-20 $\mu \mathrm{M})$ (Goddette and Frieden 1985) and competes with cofilin for binding to G-actin (Shoji, Ohashi et al. 2012).

\subsubsection{F-actin stability and neurodegeneration}

The actin cytoskeleton plays an essential role in diverse cellular processes ranging from motility to division. A key control point in the cycle of F-actin assembly is the pool of actin binding proteins that mediates F-actin turnover in response to several signaling factors. Nevertheless, the stability and/or plasticity of the filament network is vital for particular cell functions. One of these cases is the synaptic process into the neuronal spines (McConkey BJ 2002). 


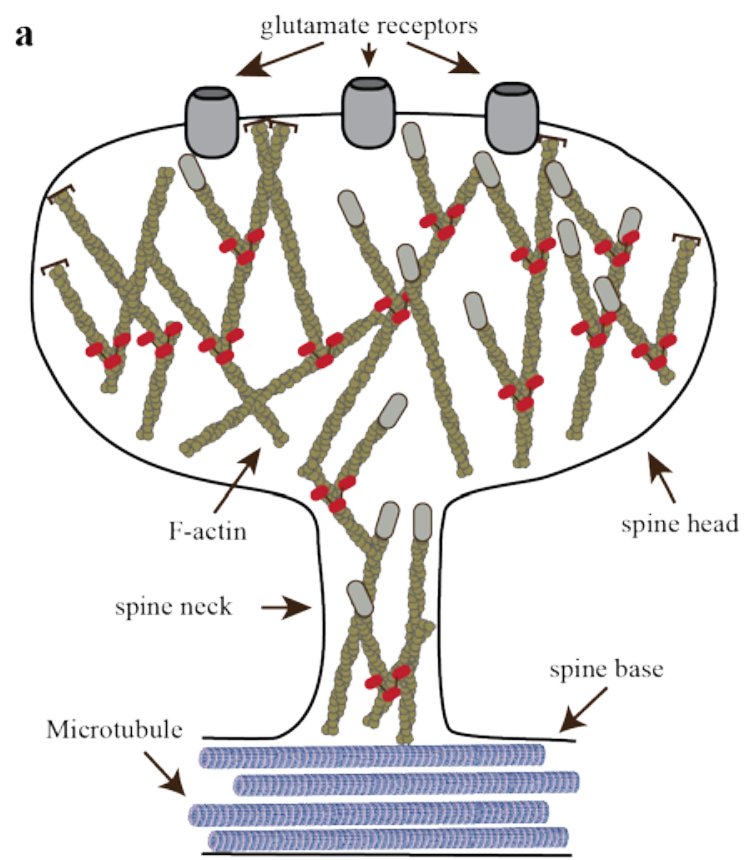

$\sim$ capping proteins $\bigcirc$ polymerizing $(+)$ end b

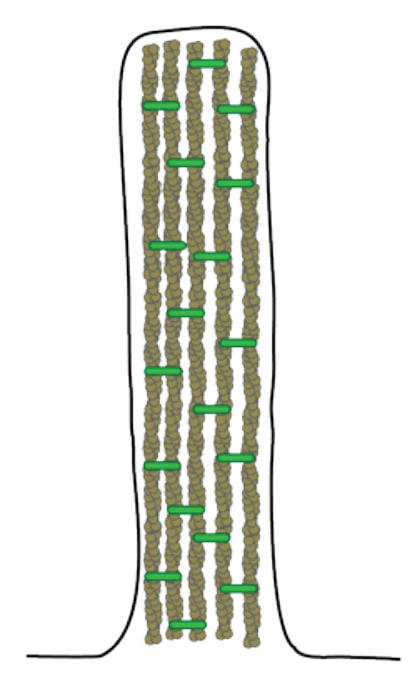

conventional filopodium

Arp2/3 complex $\quad$ cross-linking ptotein

Figure 1.11. Actin organization in neuronal spines and filopodium.

(a) Stability of dendritic spines depends on the stability of F-actin network which is modulated for the presence of capping and severing proteins like the Arp2/3 complex. In filopodium actin filaments organizes up more in bundles of parallel filaments thanks to the presence of several cross linking proteins as shown in (b).

Early electron microscopy studies have shown that actin is the major cytoskeletal component of dendritic spines (Tang, Huang et al. 2016). Provided that chemical synapses regulate the electric communication within neural networks, a precise control of the development and connectivity of synapses is critical for accurate neural network activity and normal brain function. Actin stabilizes post-synaptic proteins in mature spines (Viegas, Manso et al. 2011) and modulates spine head structure in response to postsynaptic signaling (Ni and Scheraga 1994) as shown in Figure 1.11a. The actin cytoskeleton within a spine is dynamic (Fischer, Kaech et al. 1998), although it also contains a small population of relatively stable actin filaments (Star, Kwiatkowski et al. 2002).

In migrating cells, the barbed ends of F-actin induce cell shape changes by pushing the plasma membrane forming sheet- and rod-like extensions termed lamellipodia and filopodia, respectively (Macchi, Rudd et al. 2016). Spines contain short and highly ARP2/3-promoted branched actin filaments(van Zundert, Rodrigues et al. 2016), whereas filopodia consist of long, 
unbranched, actin filaments arranged in tight, unipolar, parallel bundles (Figure 1.11b) (Gronenborn 1983).

In mature spine, the base, neck, and head all consist of a mixture of branched and linear actin filaments; the neck contains different ratios of both linear and branched filaments, whereas most branched actin filaments localize to the distal regions of the spine head ( $\mathrm{Ni}$ 1994). Actin filaments in the spine head are very dynamic and show a high turnover by continuous treadmilling (Post 2003). In this way the actin cytoskeleton of mature dendritic spines, especially of the spine head, seems like actin structures found in lamellipodia. Organization of F-actin inside spines determines the size of the spine head, which is thought to be related to the function of the spine. The largest ones are memory-related while the small ones are learning-related spines (Kasai, Matsuzaki et al. 2003)

As reviewed by Peter Penzes and Jon-Eric VanLeeuwena (Penzes and VanLeeuwen 2011) depolymerization of actin leads to spine loss as well as loss of glutamate receptors from synaptic sites. Synapse loss is a prominent and consistent finding in postmortem tissue samples from patients diagnosed with AD (Ozer and Halpain 2000, Whiteman, Gervasio et al. 2009).

The connection between F-actin stability and spine durability is increasingly recognized (Calabrese, Wilson et al. 2006, Makioka, Yamazaki et al. 2014). In addition, the dynamics of the F-actin network is controlled by members of the Rho-family GTPases, a subgroup of the Ras superfamily of GTPases (Mayer and Meyer 1999, Carlomagno 2005) through transmembrane signaling mechanisms. This signaling mechanism regulates the function of different proteins that regulates F-actin turnover. Once this mechanism is disrupted, F-actin spine stability is compromised leading to memory impairment and neuronal degeneration.

\subsection{Tau and actin interaction}

Although Tau is considered a microtubule-associated protein it can also be considered an actin binding protein. Either in vivo or in vitro conditions Tau can bind to both G-actin and F-actin (He, Wang et al. 2009, Zempel, Luedtke et al. 2013). Tau-induced actin-rich rods were found to induce neurodegeneration in Drosophila neurons (Fulga, Elson-Schwab et al. 2007). In the same study, Tau-induced neurotoxicity was found to be associated with a panneural increase in F-actin levels, while G-actin levels remained unchanged. 
It has also long been reported that Hirano bodies, which are actin-rich paracrystalline inclusions, are found in brain histopathological samples of Alzheimer's disease and related Tauopathies (Dominguez, Boelens et al. 2003, Makioka, Yamazaki et al. 2014). Further supporting information about the effect of Tau on actin dynamic it was recently proved by fluorescence microscopy that Tau-induced remodeling of the actin cytoskeleton is involved in plasma membrane blebbing (Torres-Cruz, Rodriguez-Cruz et al. 2016)

\subsubsection{Tau regions involved in binding to actin}

Several authors reported Tau and actin interaction from some time ago (Griffith and Pollard 1978, Pedrotti, Colombo et al. 1994). In 1990, Isabel Correas et al, showed that an amino acid sequence of Tau involved in the binding of Tubulin is also involved in actin binding (Merlini, Wanner et al. 2016). In the analysis they used affinity chromatography of G-actin on a column containing the synthetic peptide, and the co-sedimentation and co-localization of F-actin and the peptide. Furthermore, Yamauchi et al suggested that the interaction of Tau and F-actin is weaker than in case of microtubule although in both cases interaction has an electrostatic nature (Yamauchi and Purich 1993).

In 1993 Moraga et al found by means of electron microscopy that even sort Tau fragments from the MBD are able to bind actin and promote bundles formation (Moraga, Nunez et al. 1993). Later it was found that the C-terminal of Tau associates with actin in different PC12 while the Nterminal part may play a regulatory role in this process (Yu and Rasenick 2006). MAP2 also bind to actin filaments by their microtubule-binding domains while in case of Tau the proline-rich domain is also involved in binding (Pedrotti, Colombo et al. 1994, He, Wang et al. 2009). A synthetic Tau peptide consisting of one microtubule-binding sequence binds to both G- and Factin, but could not bundle actin filaments. This observation suggests that more than one microtubule-binding sequence of Tau is required to bundle actin filaments (Merlini, Wanner et al. 2016).

Molecular dissection studies revealed that the actin-binding site on MAP4 is situated at the Cterminal part of the proline-rich region, where the microtubule-binding site is also located (Correas, Padilla et al. 1990). Electron microscopy revealed that the MAP4-bound actin filaments become straighter and larger and that the number of actin bundles increases with greater concentrations of added MAP4 fragment. A multiple sequence alignment of the prolinerich regions of MAP4 and Tau revealed two putative actin-binding consensus sequences. 


\subsection{Tau-mediated microtubule F-actin interaction}

Cytoskeletal network is a very important for cells support and motility. The cytoskeleton is composed of three types of polymers: microtubules, actin, and intermediate filaments. Each of these polymers is associated with a large number of accessory proteins, which can regulate the assembly properties of these polymers mediating interactions among themselves as well as with other cellular structures, including plasma membrane, organelles, and chromosomes.

Interaction between actin filaments and microtubules is important for various cellular processes such as cell division, vesicle and organelle transport, axonal growth and migration (Detmers, Weber et al. 1981). F-actin and MTs can be cross-linked either directly by cross-linking proteins or indirectly via protein complexes or signaling molecules. Interactions mediated by cross linking proteins are usually considered to be static interactions, whereas the ones mediated by motor protein complexes or signaling molecules are thought to be dynamic in nature.

MAPs are key players in mediating interactions between MTs and F-actin (Spears, Furgerson et al. 2014). For instance, Tau proteins mediate interactions between microtubules and actin in the neuronal growth cones where Tau-mediated cytoskeletal interactions promote morphological changes. During axonal development, Tau accumulates into the distal ends of the axon depending on the MT and F-actin organization (Yasunaga and Wakabayashi 2001) while the presence of Tau is also required for reorganization of actin and MTs for the formation of cellular processes in insect Sf9 cells (Mandelkow and Mandelkow 1995).

Combined differential centrifugation and Co-sedimentation assays showed that Tau simultaneously binds to microtubules and actin filaments while life TIRF microscopy revealed that Tau coordinates dynamic microtubules and actin filaments (Elie, Prezel et al. 2015). Although the Tau-mediated interaction between MTs and F-actin became a common factor between scientists nowadays (Griffith and Pollard 1982, Selden and Pollard 1983) the mechanism of this interaction is still enigmatic.

\subsection{Protein NMR Spectroscopy}

Nuclear magnetic resonance (NMR) and X-ray crystallography are the most popular methods that can be applied to the study of three-dimensional molecular structures of proteins at atomic 
resolution. Unlike X-ray crystallography NMR spectroscopy allows to investigate the protein structures in nearly physiological conditions. In addition NMR spectroscopy is a very useful method for the study of kinetic reactions and properties of proteins at the atomic level. In contrast to most other methods, NMR spectroscopy studies chemical properties by studying individual nuclei. It can be applied to structure determination of proteins in the size range between 5 and $25 \mathrm{kDa}$. Traditionally NMR spectroscopy has been limited to relatively small proteins or protein domains. This is in part caused by problems resolving overlapping peaks in larger proteins, but this has been alleviated by the introduction of isotope labeling and multidimensional experiments.

\subsubsection{Relevance of structure determination using NMR}

In nature, many biological processes are regulated by protein interactions. For having more insight in understanding the process that undergoes proteins interaction, structural analysis at atomic resolution is very useful. NMR, X-ray crystallography and more recently Cryo-EM are the widely used techniques for structural analysis of biomolecules. In case of molecules of high molecular weight X-ray crystallography is most suitable if crystallization is possible.

For a characterization of internal dynamics of biomolecular structures, NMR provides direct, quantitative measurements of the frequencies of a certain energy motional process. In comparison, X-ray structure determination may include an outline of the conformational space covered by high frequency structural fluctuations. Furthermore, neutron diffraction in single crystals and NMR in solution can both be employed for studies of the exchange of labile protons, thus potentially enabling direct comparison of molecular dynamics in the different states.

The study of the protein-protein interactions is becoming more relevant to study molecular complexes. The involvement of protein interactions with its partner protein, peptides and drugs makes it important to study them at a molecular level which thereby helps in understanding the molecular basis of diseases.

\subsubsection{NMR-based Structure determination}

The conventional 2D ${ }^{1} \mathrm{H}$ NMR methods like Total Correlation Spectroscopy (TOCSY), Nuclear Oberhausen Effect Spectroscopy (NOESY) and the typical 3D experiments like HNCA, HNCACO, HNCACB etc. are very useful in NMR spectrum assignment. Nevertheless this 
method becomes ineffective when assignment of proteins with molecular masses greater than approximately $30 \mathrm{kDa}$ is required. The number of hydrogen atoms present in proteins resonances, the correlation time $(\tau c)$ of the protein and the line-widths of the NMR increases approximately linearly with molecular mass.

One of the approaches that may help is reducing the unfavorable transverse relaxation pathways. The Total Relaxation Optimization Spectroscopy (TROSY) experiment (Biernat, Wu et al. 2002) provides one illustration of this principle. Its implementation, together with other methodologies brought the NMR size limitation near 100 kDa (Knops, Kosik et al. 1991, Gustke, Trinczek et al. 1994).

Protein deuteration is another approach that together with TROSY or 3D NMR-based experiments is sometimes necessary. The two commonly used labeling strategies are perdeuteration and random fractional deuteration. Perdeuteration is the one that replaces $\sim 99 \%$ of all carbon-bound hydrogen atoms with deuterium atoms. Random fractional deuteration describes the distribution of deuterium throughout all protein molecules in the sample. For example, when a protein is $60 \%$ randomly deuterated, on average, $60 \%$ of the protons in the sample have been replaced by deuterium, but this $60 \%$ is not the same in each molecule (Butner and Kirschner 1991).

NMR-based 3D structure determination of biomolecules is usually performed by distance and geometry calculations combined with simulated annealing (Andronesi, von Bergen et al. 2008, Daebel, Chinnathambi et al. 2012). Structural constraints can be derived from NOES, J-coupling, Cross-correlated Relaxation (CCR), Residual Dipolar Couplings (RDC) or Pseudo Contact Shift (PCS) into the Paramagnetic Resonance Enhancement (PRE) approach. The decision on what method should be used will depend on the time scale of the chemical or conformational exchange processes.

For studying protein-ligand interaction the analysis can be performed by focusing either in the signal resonances of the protein or in the one from the ligand. In case the target is isotopically labeled and its size is suitable for NMR, the mapping of the binding site is possible by means of the Heteronuclear Single Quantum Correlation (HSQC) experiment (Goode and Feinstein 1994). The same approach can be used for the target in case that the isotopically labeling is possible. When the labeling is not feasible other methods like Saturation Transfer Difference (STD)(Viegas, Manso et al. 2011), transferred NOE (tr-NOE), transferred RDC (tr-RDC) and 
transferred CCR (tr-CCR) can be used. These methods are strongly depending not only on the $\tau \mathrm{c}$ of the ligand but also on the affinity range of the interaction (millimolar range).

\subsection{Aim of the study}

In this chapter we have described the importance of the interaction of the microtubule-associated protein Tau with cytoskeletal actin for cell functionality. Any disturbance of this interaction may contribute to neuronal toxicity and therefore neurodegeneration. Consistent with this hypothesis, the mechanism of Tau toxicity might involve not only the microtubule system but also interference with other cellular machines and compartments such as molecular chaperones, the nucleus and the actin cytoskeleton (Alonso, Beharry et al. 2016).

Although the interaction between Tau and actin has been studied for several years, the nature of interaction and molecular mechanisms involved are still unclear. It is important to know which region or regions of Tau are involved in binding and whether there is change in conformation of Tau upon binding to actin. In order to establish the molecular mechanism of Tau-mediated dysregulation of F-actin and the actin network, it is crucial to identify the binding site of Tau on the actin surface.

The intrinsically disordered nature of Tau in solution as well as the dynamic nature of the Tauactin interaction, where even in the bound state part of Tau remains flexible, limits the use of Xray crystallography to investigate the structure of Tau bound to actin. Therefore we decided to use NMR spectroscopy as the major tool, because NMR spectroscopy allows the description of even highly dynamic proteins and protein complex at high resolution.

Besides the ability of MAPs to bind to and regulate the dynamic instability of microtubules, MAPs bundle F-actin and cross-link the cellular cytoskeleton formed by microtubules and actin filaments. Little is known, however, about the molecular nature of Tau-mediated cross-linking of MTs and F-actin. The aim of my PhD thesis was therefore to provide detailed structural insight into the Tau/F-actin complex, the involved binding sites, the mechanism of F-actin bundling and cross-linking of the two major cytoskeleton components. 


\section{Materials and Methods}

\subsection{Buffers}

Table 2.1. Names and descriptions of all the buffers used in this study

\begin{tabular}{|c|c|}
\hline Buffers names & Buffers content \\
\hline $\begin{array}{l}\text { General actin buffer } \\
\qquad(\mathrm{GAB})\end{array}$ & $5 \mathrm{mM}$ TrisHCl pH 8.0, 0.2mM CaCl $2,0.2 \mathrm{mM}$ ATP, 0.5mM DTT \\
\hline $\begin{array}{l}\text { Polymerization buffer } \\
\qquad(\mathrm{PB})\end{array}$ & 100mM TrisHCl pH 7.5, 20mM $\mathrm{MgCl}_{2}, 500 \mathrm{mM} \mathrm{KCl,} \mathrm{10mM} \mathrm{ATP}$ \\
\hline NMR buffer & $\begin{array}{l}50 \mathrm{mM} \mathrm{NaH}_{2} \mathrm{PO}_{4} / \mathrm{Na}_{2} \mathrm{HPO}_{4} \mathrm{pH} 6.8,10 \mathrm{mM} \mathrm{NaCl}, 1 \mathrm{mM} \text { DTT, } 10 \% \\
(\mathrm{v} / \mathrm{v}) \mathrm{D}_{2} \mathrm{O}\end{array}$ \\
\hline Loading buffer blue (2X) & $\begin{array}{l}\text { 0.5M Tris-HCl (pH 6.8), 4.4\% (w/v) SDS, 20\% (v/v) glycerol, 2\% } \\
\text { (v/v) 2-mercaptoethanol, and bromophenol blue in distilled water }\end{array}$ \\
\hline $\begin{array}{l}\text { Lysis buffer } \\
\text { (LB): }\end{array}$ & $\begin{array}{l}50 \mathrm{mM} \text { Mes, } 500 \mathrm{mM} \mathrm{NaCl}, 1 \mathrm{mM} \mathrm{MgCl} 2,1 \mathrm{mM} \text { EGTA, } 5 \mathrm{mM} \\
\text { DTT, pH } 6.8\end{array}$ \\
\hline FPLC buffer & $\begin{array}{l}20 \text { mMMes, } 50 \text { mM NaCl, } 1 \text { mM EGTA, } 1 \text { mM MgCl } 2,2 \text { mM DTT, } \\
0.1 \text { mM PMSF, pH } 6.8\end{array}$ \\
\hline $\begin{array}{l}\text { Elution buffer } \\
\qquad \text { (EB) }\end{array}$ & $\begin{array}{l}20 \mathrm{mM} \text { Mes, } 1 \mathrm{M} \mathrm{NaCl}, 1 \mathrm{mM} \text { EGTA, } 1 \mathrm{mM} \mathrm{MgCl}_{2}, 2 \mathrm{mM} \text { DTT, } \\
0.1 \mathrm{mM} \text { PMSF, pH } 6.8\end{array}$ \\
\hline $\begin{array}{l}\text { Separation buffer } \\
\text { (SB) }\end{array}$ & $\begin{array}{l}137 \mathrm{mM} \mathrm{NaCl}, 3 \mathrm{mM} \mathrm{KCl}, 10 \mathrm{mM} \mathrm{Na}_{2} \mathrm{HPO}_{4}, 2 \mathrm{mM} \mathrm{KH}_{2} \mathrm{PO}_{4}, \mathrm{pH} \\
\text { 7.4, } 1 \mathrm{mM} \text { DTT }\end{array}$ \\
\hline $\begin{array}{l}\text { Boiling-extraction buffer } \\
\text { (BEB) }\end{array}$ & $\begin{array}{l}50 \text { mM MES, } 500 \mathrm{nM} \mathrm{NaCl,} \mathrm{1mM} \mathrm{MgCl}_{2} \text {, } 1 \text { mM EGTA, } 5 \mathrm{mM} \\
\text { DTT, pH } 6.8\end{array}$ \\
\hline Phosphorylation buffer & $\begin{array}{l}25 \text { mM 2-[4-(2-hydroxyethyl)piperazin-1-yl]ethanesulfonic acid (pH } \\
\text { 8.0), } 100 \text { mM NaCl, } 5 \text { mM MgCl2, } 2 \text { mM EGTA, } 1 \text { mM DTT, } 1 \text { mM } \\
\text { benzamidine, } 0.5 \text { mM phenyl - methanesulfonyl fluoride (PMSF), } \\
\text { and } 1 \text { mM ATP }\end{array}$ \\
\hline
\end{tabular}




\subsection{Chemical compounds}

Cytochalasin D (Cat. No. 10-2071) and latrunculin B (Cat. No. 10-4303) were bought from Focus Biomolecules company whereas 1,4-Dithiothreitol (DTT) (Cat. No. D9779-50G) and Adenosine triphosphate (ATP) (Cat. No. 10127531001) were purchased from Sigma Aldrich.

\subsection{Protein preparation and purification}

Cloning, expression and purification of wild-type (wt) hTau40, MARK2-phosphorylated hTau40 (pTau) and K18 were performed by Dr. Jacek Biernat in the lab of Prof. Dr. Mandelkow, DZNE, Bonn, Germany. MARK2-phosphorylated Tau was prepared as previously described in our lab (Schwalbe, Biernat et al. 2013). At the end of the phosphorylation process the sample was heated to denature the kinase.

\subsubsection{Protein expression in E.coli.}

Human Tau and K18 were expressed in the vector pNG2 (a derivative of pET-3a; Merck) in E. coli strain BL21(DE3) as described (Gustke, Trinczek et al. 1994). The expressed proteins were purified from bacterial extract by using the heat stability of Tau in combination with FPLC SPSepharose ion exchange chromatography (GE Healthcare, Freiburg).

Bacterial pellets were resuspended in BEB supplemented with protease inhibitor cocktail. Cells were disrupted with a French pressure cell and subsequently boiled for $20 \mathrm{~min}$. The soluble extract was isolated by centrifugation and the supernatant was dialyzed twice against FPLC buffer, and loaded onto a FPLC SP-Sepharose column. Proteins were eluted using a linear gradient of EB. Tau breakdown products were separated in a second chromatography step by using a Superdex G200 column (GE Healthcare) with the Separation Buffer.

\subsubsection{Protein expression with isotope labeling}

To label the Tau proteins with ${ }^{15} \mathrm{~N}$ isotope, the E. coli cultures were grown in a M9 minimal medium with ${ }^{15} \mathrm{NH}_{4} \mathrm{Cl}$ (1 g/liter) (Eurisotop, Saarbrücken). Subsequently, protein samples 
uniformly enriched in ${ }^{15} \mathrm{~N}$ were prepared by growing $E$. coli bacteria in minimal medium containing $1 \mathrm{~g} /$ liter of ${ }^{15} \mathrm{NH}_{4} \mathrm{Cl}$ exclusively.

For isotope labeling of Tau proteins for NMR experiments, a rapid and efficient approach for preparing isotopically labeled recombinant proteins was used as reported by Marley, Lu et al. (Balaram, Bothner-By et al. 1972). This production method generates cell mass using unlabeled rich media followed by exchange into a small volume of labeled media at high cell density. Cells were induced with $0.8 \mathrm{mM}$ IPTG following a short period for growth recovery and unlabeled metabolite clearance.

Example for $1 \mathrm{~L} \mathrm{E}$. coli culture, bacteria were grown in $1 \mathrm{~L}$ of Luria broth at $37^{\circ} \mathrm{C}$, upon reaching the optical cell density of 0.7 at $600 \mathrm{~nm}\left(\mathrm{OD}_{600}\right)$. The cells were pelleted, using an M9 salt solution and the pellet was resuspended in $250 \mathrm{ml}$ of isotopically labeled minimal medium. The culture was then incubated for 1 hour to allow recovery of growth and clearance of unlabeled metabolites. Afterwards protein expression was induced by addition of IPTG to a concentration of $0.8 \mathrm{mM}$. Cells were harvested after 4 hours incubation. The expressed proteins were purified from bacterial extracts by methods described for non-isotopic expression.

\subsubsection{Standard 5 x M9 minimal medium without nitrogen source}

For 1L 5 x M9 salts:

$64 \mathrm{ga}_{2} \mathrm{HPO}_{4}-7 \mathrm{H} 2$

$15 \mathrm{~g} \mathrm{KH}_{2} \mathrm{PO}_{4}$ and $2.5 \mathrm{~g} \mathrm{NaCl}$

Add $\mathrm{H}_{2} \mathrm{O}$ to final volume $1 \mathrm{~L}$ and autoclave

\subsubsection{Preparation of $500 \mathrm{~mL}$ M9 minimal medium}

$100 \mathrm{~mL} 5$ x M9 salts

$1 \mathrm{~mL} 1 \mathrm{M} \mathrm{MgSO}_{4}$

$50 \mu \mathrm{L} 1 \mathrm{M} \mathrm{CaCl}_{2}$

1mL 100 x Basal Medium Eagle Vitamin Solution (Gibco)

$2.5 \mathrm{~mL}$ filter sterilized $\mathrm{NH} 4 \mathrm{Cl}(0.2 \mathrm{~g} / \mathrm{mL})$ or $0.5 \mathrm{~g}$ dry

10 mL 20\% d-glucose or 2g dry 


\subsection{Tau constructs and synthetic peptides}

The used full-length Tau as well as K18 and the synthetic Tau peptides are represented in Figure 2.1. HTau40 is the largest human Tau isoform in the central nervous system with 441 residues. Tau contains two amino terminal inserts (N1 and N2), a proline-rich region divided in two sub regions (P1 and P2) and four repeats in the carboxyl terminal part and an additional repeat $\mathrm{R}^{\prime}$. K18 represents the 4-repeat C-terminal assembly domain and contains 128 amino acids with amino terminal methionine. K18 was generated using PCR on the template of hTau40 DNA sequence provided with NdeI and BamHI restriction enzyme recognition sites.

Projection domain
(15

Figure 2.1. Tau protein and Tau constructs used in this study.

Top panel represent the largest isoform (hTau40, 2N/4R) in the human brain with 441 amino acids. K18, a 4-repeat construct at the C-terminal part of Tau comprises residues Q244 to E372. In addition, the Tau peptides Tau(211-242), Tau(254-290) and Tau(292-319) were used. The sequenes of the used peptides as well as ciboulot are also shown

Tau and ciboulot peptides were synthesized by Kerstin Overkamp in the Department of NMR-based Structural Biology, Max Planck Institute for Biophysical chemistry, Göttingen. Synthesis was based on standard Fmoc-solid-phase peptide synthesis using an ABI 433A synthesizer (Applied Biosystem). Peptides were synthesized with acetyl- and amide protein groups at the $\mathrm{N}$ - and C-termini, respectively. Peptides were further purified by reversed-phase HPLC and the pure product was lyophilized. 


\subsection{Other proteins}

\subsubsection{Human actin}

Non-muscle human actin was bought from Cytoskeleton Company (Catalog No. APHL99). Every milligram of lyophilized actin was reconstituted to $10 \mathrm{mg} / \mathrm{mL}$ by adding $100 \mu \mathrm{l}$ of distilled water to be in the following buffer: GAB with $5 \%(w / v)$ sucrose and $1 \%(w / v)$ dextran. The sample was aliquoted and kept at $-20^{\circ} \mathrm{C}$ for later usage.

\subsubsection{F-actin assembly}

A total of $20 \mu \mathrm{L}$ of sample containing $0.4 \mathrm{mg} / \mathrm{ml} \mathrm{G}$-actin were prepared in $\mathrm{GAB}$ and supplemented with $0.2 \mathrm{mM}$ ATP. The sample was then incubated on ice for $1 \mathrm{~h}$ and centrifuged at 14000 rpm for 20 minutes, in order to remove oligomers that might have formed during storage. Subsequently, the sample was supplemented with $20 \mu \mathrm{L}$ of $\mathrm{PB}$ and incubated at room temperature for $1 \mathrm{~h}$ to promote the polymerization of actin monomers. Subsequently, the sample was centrifuged at $100000 \mathrm{~g}$ for $1 \mathrm{~h}$ at $4{ }^{\circ} \mathrm{C}$ by using a Beckman Airfuge (rotor TLA 100.3) collecting the filaments at the bottom of the ultracentrifuge tube. Filaments were then resuspended in GAB or NMR Buffer according to the desired experiment. Actin quantification was performed by the precision red advanced protein assay reagent (Cytoskeleton, Catalog No.ADV02-A) as suggested by Cytoskeleton, Inc. Here the optical density of the conjugated actin was recorded at $600 \mathrm{~nm}\left(\mathrm{OD}_{600}\right)$ on a spectrophotometer (BioSpectrometer ${ }^{\circledR}$ kinetic, Eppendorf). The protein concentration was then estimated according to:

$1.00 O D_{600}=100 \mu g$ protein per $\mathrm{mL}$ reagent per cm light path-length

The measured absorbance was multiplied by 100 to achieve $\mu$ g of protein per $\mathrm{mL}$ of the original sample. The procedure was repeated twice, one for determining the concentration of actin before polymerization and another time to estimate the efficiency of the polymerization process by determining the actin concentration in the supernatant after centrifugation.

\subsubsection{Human cofilin}

Human cofilin (Catalog No.CF01) was bought from Cytoskeleton Company. $100 \mu \mathrm{g}$ of cofilin powder were resuspended in $20 \mu \mathrm{l}$ of distilled water to have $5 \mathrm{mg} / \mathrm{mL}$ of protein in a final buffer 
of $10 \mathrm{mM}$ Tris $\mathrm{pH}$ 8.0, $10 \mathrm{mM} \mathrm{NaCl}, 5 \%$ sucrose and 1\% dextran. The sample was subsequently dialyzed against GAB or NMR buffer to be used for electron microscopy or NMR experiments, respectively. For dialysis, a concentrator with a cutoff of $3 \mathrm{kDa}$ (Amicon Ultra-0.5 mL Centrifugal filters) was used.

\subsection{F-actin binding assay}

For binding assays, NBD-conjugated actin from $\alpha$-skeletal muscle was bought from Hypermol (www.hypermol.com). In this experiment, 7-chloro-4-nitrobenzeno-2-oxa-1,3-diazole (NBD)labeled G-actin was polymerized starting from $0.4 \mathrm{mg} / \mathrm{ml}$ of the conjugated actin. To provide stability to the filaments, the sample was supplemented with 10-fold excess of phalloidin (catalog no. P2141, Sigma-Aldrich). NBD-labeled actin was polymerized in presence of PB as explained before. Next, $0.25 \mu \mathrm{M}$ of NBD-labeled F-actin was incubated with increasing amounts of Tau or Tau constructs. The change in fluorescence of NBD was followed in a Cary Eclipse fluorescence spectrophotometer (Agilent technology). Excitation and emission wavelengths were set to 480 and $530 \mathrm{~nm}$, respectively. A total of three experiments were carried out at $25{ }^{\circ} \mathrm{C}$ in GAB and the standard deviation was estimated. The normalized decrease in fluorescence (equation 1) was analyzed and fitted by using equation 2 (Ojala, Paavilainen et al. 2002, Mattila, Quintero-Monzon et al. 2004):

$E=\frac{\left(F-F_{0}\right)}{\left(F_{\max }-F_{0}\right)}$

$E=\frac{1}{2} C+\frac{1}{2} Z-\frac{1}{2} \sqrt{(C+Z)^{2}-4 Z}$

, where

$Z=\frac{[\text { partner }]}{[\text { F-actin }]} \quad$ and $\quad C=1+\frac{K d}{[F-\text { actin }]}$

A reference experiment with phalloidin-stabilized NBD-labeled F-actin in the absence of additional binding partners was performed, in order to control the stability of the filaments over the duration of the experiment. 


\subsection{Transmission electron microcopy}

Electron microscopy was performed by Gudrun Heim and Dr. Dietmar Riedel at the Facility for Transmission Electron Microscopy, Max Planck Institute for Biophysical Chemistry, Göttingen. Actin filaments were initially diluted to $10 \mu \mathrm{M}$ in GAB. Samples were bound to a glow discharged carbon foil covered copper grid. After washing of the grids using $\mathrm{ddH}_{2} \mathrm{O}$, the samples were stained using 1\% uranyl acetate. Electron micrographs were recorded at room temperature using a CM 120 transmission electron microscope (FEI, Eindhoven, and The Netherlands) and a TemCam F416 CMOS camera (TVIPS, Gauting, Germany).

\subsection{Co-sedimentation assay}

In this experiment mixtures of F-actin with Tau (1:1 molar ratio) or Tau-peptides (1:10 actin:Tau-peptide molar ratio) were incubated at room temperature for 30 minutes. In case of shorter peptides such as Tau(254-268), a 1:30 ratio was prepared. Centrifugation was performed in two separated steps in a Beckman Airfuge (rotor TLA 100.3). First a spin rate of $5000 \mathrm{~g}$ was used during 15 minutes, in order to collect bundles of F-actin (PB) at the bottom of the tube. The remaining sample was centrifuged at $100000 \mathrm{~g}$ during $1 \mathrm{~h}$ for collecting actin filaments (PF). The final supernatant (SN) was also kept for later usage. Pellets were resuspended in $80 \mu \mathrm{l}$ of GAB. For electron microscopy, $25 \mu \mathrm{l}$ of sample were taken while the remaining $55 \mu \mathrm{l}$ were loaded in a 4-20\% gradient SDS gel upon colored with Bromophenol Blue and denatured with SDS and heat. Polyacrylamide gels were treated with Coomassie (Brilliant) Blue to stain the proteins and later visualization on the Molecular Imager ${ }^{\circledR}\left(\mathrm{Gel}\right.$ Doc ${ }^{\mathrm{TM}} \mathrm{XR}+$; Biorad).

\subsection{MTSL-labeling of actin}

In order to perform PRE experiments, G-actin was labeled with MTSL ((1-oxy-2,2,5,5tetramethyl-d-pyrroline-3-methyl-methanethiosulfonate), Toronto Research Chemicals) on Cysteine-374 (C374). Directly before addition of MTSL, DTT was removed from the sample by using a $2 \mathrm{~mL}$ Zeba desalting column, which had been equilibrated with GAB at pH 6.8. Next, free sulfhydryl groups were modified by incubating the sample overnight at $0{ }^{\circ} \mathrm{C}$ with a 20 -fold molar excess of MTSL. Unreacted MTSL was removed with another $2 \mathrm{ml}$ Zeba desalting 
column. Afterwards, MTSL-labeled actin was polymerized as described above, in order to obtain MTSL-labeled F-actin with the paramagnetic nitroxide tag covalently bound to C374.

\subsection{NMR spectroscopy}

\subsubsection{One-dimensional NMR experiments}

In order to test protein integrity, one-dimensional (1D) experiments were performed before doing further experiments. To this end, a standard Bruker pulse sequence (zggpw19.pl) using a 3-9-19 water gate for water suppression was used. Depending on protein concentration, a total of 64 or 128 scans were recorded, while the time domain (TD) was set to $1 \mathrm{k}$ in all cases. The 3-9-19 parameter D19, which takes into account the distance to the next excitation null, was set to 100 $\mu \mathrm{s}$.

\subsubsection{Saturation transfer difference (STD)}

Saturation transfer difference (STD) NMR is one of the most popular ligand-based NMR techniques for the study of protein-ligand interactions (Mayer and Meyer 1999, Viegas, Manso et al. 2011, Hetenyi, Hegedus et al. 2016, van Zundert, Rodrigues et al. 2016). This is a robust technique, which is focused on the signals of the ligand, without any need of processing NMR information about the receptor and only using small quantities of the unlabeled macromolecule. The method is based on the selective saturation of molecules with a high molecular weight such as microtubules and actin filaments. Subsequently the magnetization is transferred to the bound ligand, whose signals can be detected in a difference spectrum resulting from an on- and off resonance setup. A series of selective pulses was used to selectively saturate the protons of the receptor. At the same time, the receptor saturation frequency was applied at a proton frequency where no ligand resonances were observed.

When the receptor is irradiated, magnetization rapidly spreads across the receptor by means of spin diffusion, which is then transferred to the protons of the ligand at the binding interface through a cross relaxation pathway. This principle is represented in Figure 2.2. 

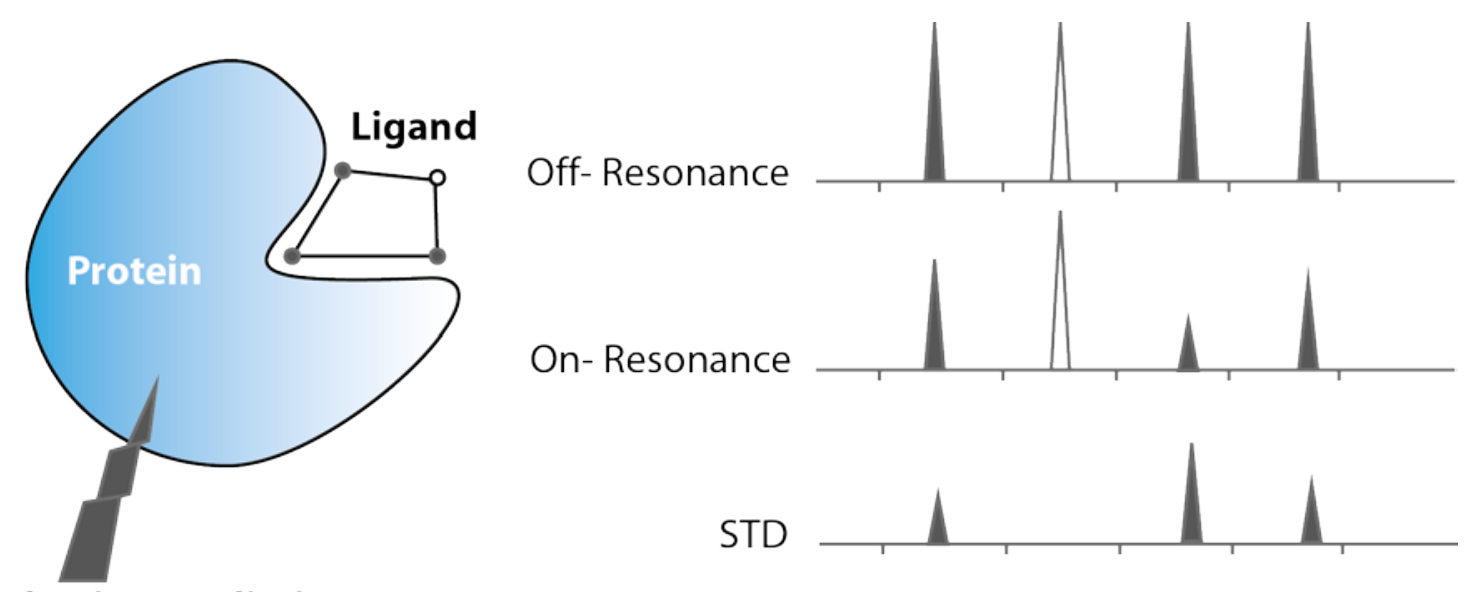

Selective Irradiation

Figure 2.2. Schematic representation of STD NMR spectroscopy.

Selective saturation transfer of magnetization from a protein to the binding region of the ligand. Protons from the ligand, which are in close proximity to the protein, will experience the STD effect (closed circles) while those which are far away from the binding site (open circle) will experience no STD effect. The intensity of the STD signal will depend on the proximity of the atom at the binding interface.

\subsubsection{Experimental procedure}

For STD-based NMR measurements, $800 \mu \mathrm{M}$ of peptides were mixed with $26.6 \mu \mathrm{M}$ F-actin in NMR buffer (molar ratio of 30:1). STD NMR spectra were acquired with and without F-actin to characterize the interaction. All STD spectra were recorded on a $700 \mathrm{MHz}$ spectrometer equipped with a cryogenic NMR probehead. NMR spectra were acquired at $278 \mathrm{~K}$ using a series of 40 equally spaced 50 ms Gaussian-shaped pulses, in order to achieve saturation of protein NMR resonances for 2 seconds. Four different on-resonance irradiation frequencies were tested and -0.5 ppm was selected, to optimally achieve the protein saturation without ligand irradiation. The off-resonance frequency was set to $60 \mathrm{ppm}$ in all cases. A total of 1024 scans were collected for each experiment with a recycle delay of $2 \mathrm{~s}$.

\subsubsection{D NMR experiments}

\subsubsection{Heteronuclear Single Quantum Coherence (HSQC)}

The Heteronuclear Single Quantum Coherence (HSQC) experiment is one of the most frequently used experiments in protein NMR spectroscopy. This method gives one peak per pair of coupled nuclei (I and X), whose two coordinates are chemical shifts of the two coupled atoms (Bodenhausen and Ruben 1980, McConkey BJ 2002). 


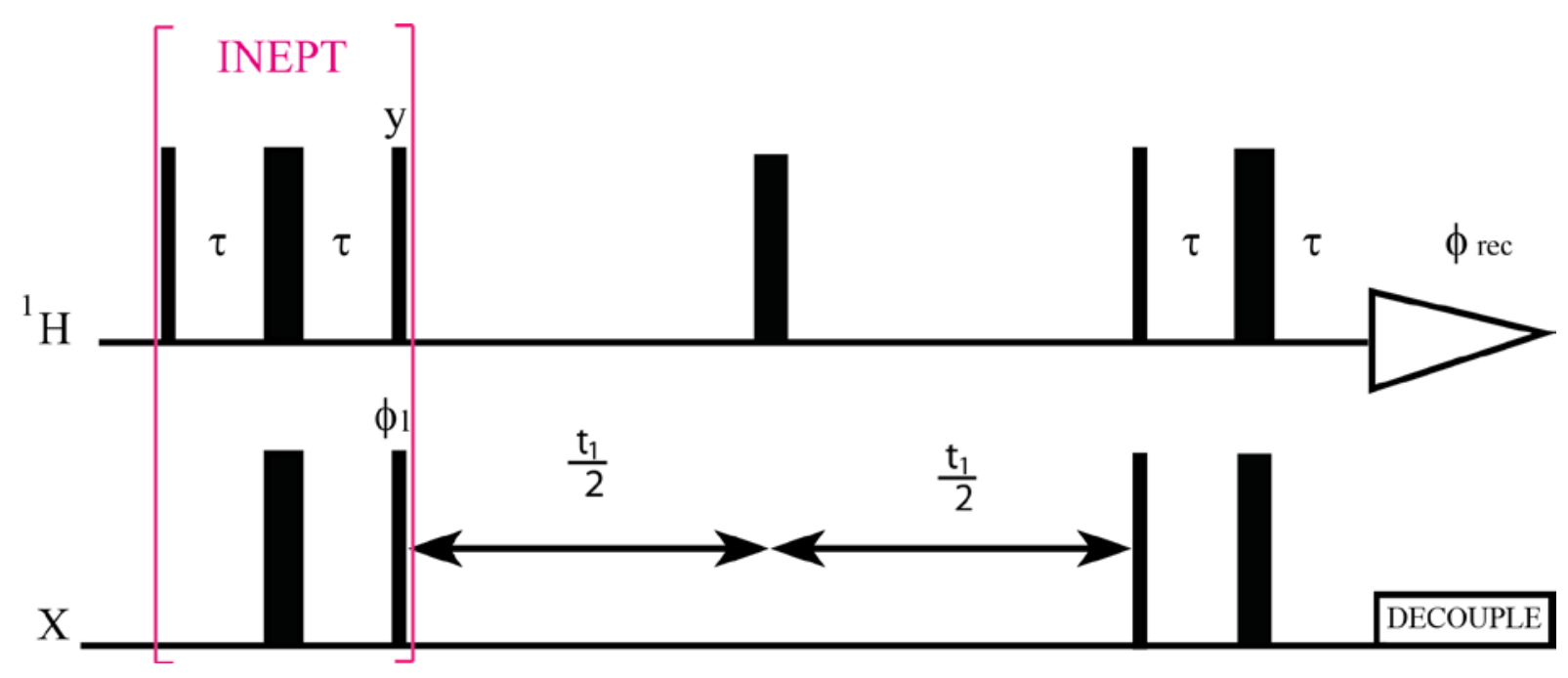

Figure 2.3. Schematic representation of a 2D HSQC pulse sequence.

An initial INEPT pulse train transfers polarization from ${ }^{1} \mathrm{H}$ to ${ }^{15} \mathrm{~N}$ or ${ }^{13} \mathrm{C}$ represented as $\mathrm{X}$. The delay “ $\tau$ ” should be defined as $1 / 4 \mathrm{~J}$ for the magnetization to be efficiently transferred to the $\mathrm{X}$ nucleous, where $\mathrm{J}$ is the coupling constant between ${ }^{1} \mathrm{H}$ and $\mathrm{X}$ nuclei. $\phi 1$ an $\phi r e c$ reprsent a fase cicling for selecting a desired mgnetization pathway. Subsequently the antiphase magnetization evolves during the variable evolution $\mathrm{t}_{1}$ period under the effect of $\mathrm{X}$ chemical shift. Heteronuclear ${ }^{1} \mathrm{H}-\mathrm{X}$ couplings are refocused by applying a $180^{\circ}{ }^{1} \mathrm{H}$ pulse at the middle of this period. A retro-INEPT pulse train converts $\mathrm{X}$ magnetization to inphase ${ }^{1} \mathrm{H}$ magnetization. Proton acquisition is performed with $\mathrm{X}$ decoupling (DECOUPLE block).

HSQC works by transferring magnetization from the "I" nucleus (usually the proton) to the "X" nucleus (usually ${ }^{15} \mathrm{~N}$ or ${ }^{13} \mathrm{C}$ ) using the Insensitive Nuclei Enhancement through Polarization Transfer (INEPT) pulse sequence. Because proton nuclei have greater equilibrium magnetization, the transfer process creates stronger signal on the second nucleus when compared to direct excitation of the " $X$ " nucleus. In a second step, the magnetization evolves to be transferred back to the ${ }^{1} \mathrm{H}$ nucleus for observation. The pulse sequence of the HSQC is schematically shown in Figure 2.3. An extra spin echo step can optionally be used to decouple the signal, simplifying the spectrum by collapsing multiplets into a single peak. For example, the ${ }^{1} \mathrm{H}_{-}{ }^{15} \mathrm{~N}$ HSQC detects correlations between ${ }^{15} \mathrm{~N}$ and ${ }^{1} \mathrm{H}$ nuclei, which are separated by one bond. In proteins each residue, except prolines, has an amide proton attached to nitrogen in the peptide bond and the HSQC provides the correlation between the nitrogen and amide proton.

Protein-protein and protein-ligand interactions can be well studied using the $2 \mathrm{D}{ }^{1} \mathrm{H}^{15} \mathrm{~N}$ HSQC experiment (Rajagopal, Waygood et al. 1997, O'Connell, Gamsjaeger et al. 2009). The binding effect can be correlated with changes in the chemical shift $(\delta)$ of the resonance and line broadening, because of changes in the chemical environment and appearance of additional 
relaxation pathways in the binding region. To this end, a $2 \mathrm{D}{ }^{1} \mathrm{H}^{15} \mathrm{~N}-\mathrm{HSQC}$ spectrum of ${ }^{15} \mathrm{~N}$ labeled protein will be recorded in the absence and presence of the unlabeled binding partner at stoichiometric or sub stoichiometric conditions. In case of the Tau/ F-actin interaction, the receptor (F-actin) is very large, interacting Tau residues experience extensive dipolar interaction and an increase in rotational correlation time, leading to severe line broadening.

2D ${ }^{1} \mathrm{H}_{-}{ }^{15} \mathrm{~N}$ HSQC experiments were recorded on Bruker spectrometers (Bruker Karlsruhe, Germany) with 800 or $900 \mathrm{MHz}$ proton frequency and equipped with a TCI cryogenic probe (Zgradient). A HSQC pulse sequence containing a 3-9-19 water gate for water suppression was used. The nitrogen carrier was set to $118 \mathrm{ppm}$ and the spectral with was $24 \mathrm{ppm}$ while for proton the carrier was set to the water resonance frequency. Spectra were acquired with 512 increments in the indirect dimension and $2 \mathrm{k}$ points in the direct dimension. For processing a zero filling of $4 \mathrm{k}$ and $1 \mathrm{k}$ in the direct and indirect dimensions were used, respectively. A sine-squared bell window function shifted either by $90^{\circ}$ or $60^{\circ}$ was applied. Data were processed using NMRPipe (Delaglio, Grzesiek et al. 1995) and analyzed using the software Sparky 3.114 (T. D. Goddard and D. G. Kneller, http://www.cgl.ucsf.edu/home/sparky).

\subsubsection{Total Correlation Spectroscopy (TOCSY)}

Total Correlation Spectroscopy (also known as HOHAHA - HOmonuclear Hartmann HAhn) (Braunschweiler and Ernst 1983, Bax and Davis 1985, Nuzillard and Massiot 1991) is useful for dividing the proton signals into groups or coupling networks, especially when the multiplets have similar chemical shifts or there is extensive second order coupling (Cavanagh, Fairbrother et al. 2007). A TOCSY spectrum yields correlations via spin-spin coupling through bond. TOCSY is often used in larger molecules with many separated coupling networks such as peptides, proteins and polysaccharides. In the TOCSY experiment, cross peaks are observed not only for nuclei, which are directly coupled, but also between nuclei which are connected through a chain of couplings. Therefore the TOCSY experiment is useful for identifying larger interconnected networks of coupled spins. Transfer of magnetization between multiple coupled spins is achieved by insertion of a repetitive sequence of radiofrequency pulses, resulting in isotropic mixing during the mixing period (Cavanagh, Fairbrother et al. 2007). In a TOCSY spectrum, cross peaks originate from protons of the same spin system. No magnetization transfer occurs between different residues, enabling identification of residue-specific spin systems (Wüthrich 1986). 


\subsubsection{Nuclear Overhauser Effect (NOE)}

The Nuclear Overhauser Effect is the most clear manifestation of the fact that two dipolarcoupled nuclei do not relax independently (Cavanagh, Fairbrother et al. 2007). When a specific nucleus is magnetically excited and its neighbor is at equilibrium, relaxation occurs between the two nuclei. Because of this relaxation, intensities of the nucleus at equilibrium state changes. The smaller the physical distance between the nucleus at the equilibrium state and the excited nucleus, the bigger the expected change. NOESY cross-peaks are originated from the dipolar couplings resulting from interactions of spins through the space and hence only depend on the distance but not on the number of intervening bonds. The NOE is characterized by the crossrelaxation rate constant $\left(\sigma_{I S}^{N O E}\right)$, where $\mathrm{S}$ and I refer to two different scalar-coupled spins. In general $\sigma_{I S}^{N O E}$ can be mathematically expressed as:

$\sigma_{I S}^{N O E}=\frac{\hbar^{2} \mu_{0}^{2} \gamma_{I}^{2} \gamma_{S}^{2} \tau_{c}}{40 \pi^{2} r_{I S}^{6}}\left(-1+\frac{6}{1+4 \omega_{0}^{2} \tau_{c}^{2}}\right)$

, where $\hbar$ is the Dirac constant, $\mu_{0}$ is the magnetic permeability at vacuum, $\gamma$ is the gyromagnetic ratio of the specific spin, $\tau_{c}$ is the correlation time and $r_{I S}$ is the distance between I and $\mathrm{S}$ spins.

In the extreme narrowing limit (i.e. $\omega \tau_{c}<<1$ ), the equation 3 is reduced to (Cavanagh, Fairbrother et al. 2007):

$\sigma_{I S}^{N O E}=\frac{\hbar^{2} \mu_{0}^{2} \gamma_{I}^{2} \gamma_{S}^{2} \tau_{c}}{8 \pi^{2} r_{I S}^{6}}$

Whereas in the spin diffusion limit (i.e. $\omega \tau_{c}>>1$ )

$\sigma_{I S}^{N O E}=\frac{\hbar^{2} \mu_{0}^{2} \gamma_{I}^{2} \gamma_{S}^{2} \tau_{c}}{40 \pi^{2} r_{I S}^{6}}$

equations 4 and 5 clearly reveal that the NOE is strongly dependent on the distance between the interacting spins. In practice a NOE cross peak can be observed only is the inter-spin distance is $\leq 6 \AA$.

The pulse sequence for the Nuclear Overhauser Effect Spectroscopy (NOESY) experiment with a 3-9-19 water gate for water suppression is shown in Figure 2.4. Initially a $\pi / 2-t_{1}-\pi / 2$ period frequency-labels the ${ }^{1} \mathrm{H}$ spins and brings the magnetization back to " $\mathrm{z}$ " axis. Subsequently magnetization transfer occurs via dipolar coupling during a period $\tau_{\mathrm{m}}$. The proper selection of $\tau_{\mathrm{m}}$ is crucial to determine the proper distances between the involved nuclei. Subsequently the 
magnetization is rotated again by a $\pi / 2$ pulse to be acquired previous suppression of the water signal by a 3-9-19 water-gate block. Upon acquisition, the system is let to reach the equilibrium during a delay $\mathrm{d}_{1}$. This delay should be around 6 times the longitudinal relaxation of the total system $\left(\mathrm{T}_{1}\right)$.

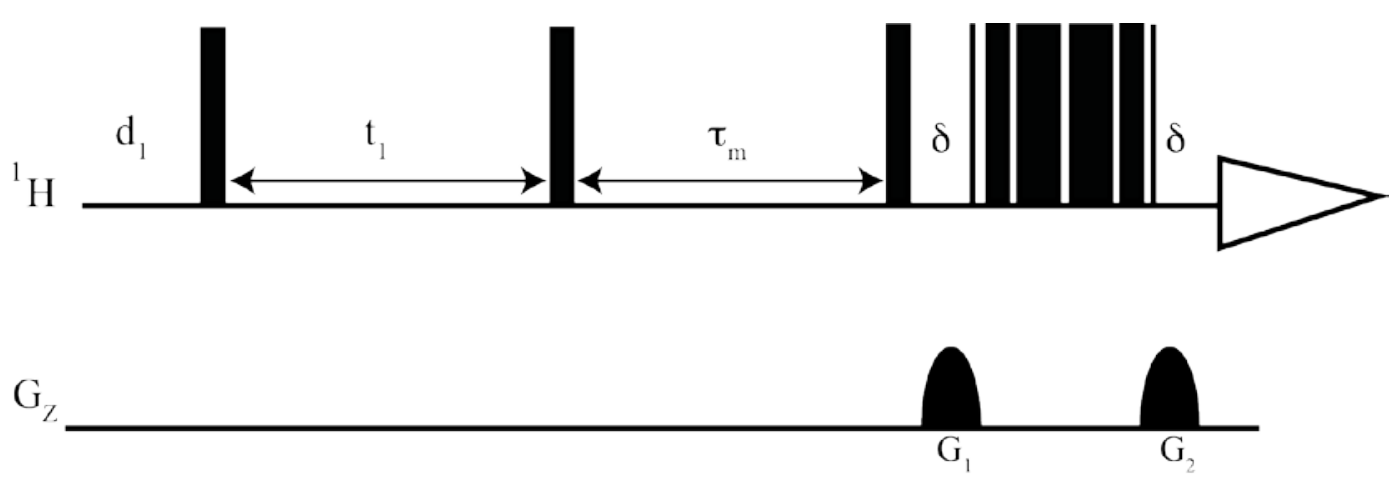

Figure 2.4. Schematic representation of a NOESY pulse sequence.

The schema represents a phase-sensitive pulse sequence of a NOESY experiment "noesygpph19" from Bruker. The first three squeares on the button panel represent 90 degree pulses which are separated by a relaxation period $\left(\mathrm{t}_{1}\right)$ where proton magnetization is rotated to the transversal plane. The mixing time $\left(\tau_{\mathrm{m}}\right)$ period is required for the dipolar-mediated relaxation to occur. Other parameters like $G_{1,2}$ and $\delta$ are required for a proper water suppression.

\subsubsection{Resonance assignment}

The unlabeled peptides Tau(211-242), Tau(254-268), Tau(254-284), Tau(254-290), Tau(292319) and Cib(D1) were assigned using two-dimensional ${ }^{1} \mathrm{H}-{ }^{1} \mathrm{H}$ TOCSY and ${ }^{1} \mathrm{H}-{ }^{1} \mathrm{H}$ NOESY spectra recorded at $278 \mathrm{~K}$, either on Bruker 800 or $900 \mathrm{MHz}$ spectrometer equipped with a cryogenic probeheads. Samples were prepared in NMR buffer at a concentration of $800 \mu \mathrm{M}$. Acquisition parameters were set to 40 transients, sweep widths of $12 \times 12 \mathrm{ppm}$ in the direct and indirect dimensions, respectively. The time domain (TD) was set to 2048 point in the F2 dimension, while 512 points were used in F1. Mixing times of $70 \mathrm{~ms}$ for TOCSY and $80 \mathrm{~ms}$ for NOESY were used. Spectra were processed using NMRPipe (Delaglio, Grzesiek et al. 1995) and analyzed using the software Sparky 3.114 (T. D. Goddard and D. G. Kneller, http://www.cgl.ucsf.edu/home/sparky). The individual spin systems were identified by using TOCSY and were sequence specifically assigned in combination with NOESY (Wüthrich 
1986). The assignments of hTau40 and K18 were performed previously in our lab (Mukrasch, Bibow et al. 2009) and were used to analyze the HSQC spectra.

\subsubsection{Transferred NOE (tr-NOE)}

The basic principle of transferred NOE (tr-NOE) was first described by Balaram, Bothner-By et al. (Balaram, Bothner-By et al. 1972) and reviewed by several other authors (Ni 1994, Post 2003). The ideal systems for the observation of tr-NOE are those where interactions occur with dissociation constants within the $\mu \mathrm{M}$ to $\mathrm{mM}$ range, resulting in exchange of the ligand between the bound and free state, which is fast on the NMR time scale (Balaram, Bothner-By et al. 1972, Clore and Gronenborn 1982).

For efficient magnetization transfer from the bound to the free state of the ligand, the following equation should be fulfilled:

$$
\left|N_{b} . \sigma_{b}\right| \gg\left|N_{f} \cdot \sigma_{f}\right|
$$

, where $\sigma$ is the cross-relaxation rate and $\mathrm{N}$ the number of molecules in the free $\left(\mathrm{N}_{\mathrm{f}}, \sigma_{\mathrm{f}}\right)$ and bound $\left(\mathrm{N}_{\mathrm{b}}, \sigma_{\mathrm{b}}\right)$ states.

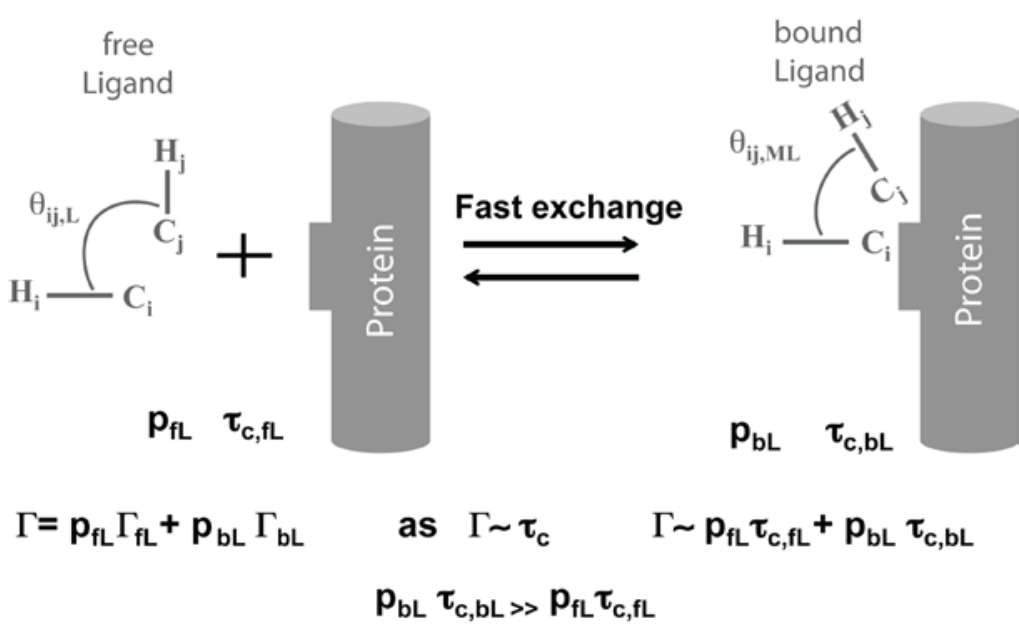

Figure 2.5. Pictorial representation of a fast exchange ligand-receptor interaction.

At the left panel the free ligand population $\left(\mathrm{P}_{\mathrm{fL}}\right)$ with its particular correlation time $\left(\tau_{\mathrm{c}}, \mathrm{fL}\right)$. At the right panel part of the free ligands bind to the protein $\left(\mathrm{P}_{\mathrm{bL}}\right)$ acquiring a bigger correlation time $\left(\tau_{\mathrm{c}}\right.$, fL $)$ because they became part of the complex wit the protein for short time. Any change in conformation upon binding will be observed as the populated weighted averaging of the NMR observable NOE.

For a successful experiment, special attention has to be addressed to the sample preparation. If the ligand-to-protein ratio is too high, there will be more ligand free in solution and, as a result, 
its corresponding positive NOE may lead to a significant reduction or even cancelation of the trNOESY enhancements coming from the negative NOE developed by the small fraction of bound ligand. On the other hand if the ligand-to-protein ratio is too low all the ligands will be in bound state and therefore the NMR signal would be too low. The cartoon representation of the tr-NOE concept is presented in Figure 2.5.

As represented in Figure 2.5 the observation of tr-NOE relies on the existence $\tau_{\mathrm{c}}$ for the free and bound ligand. Free ligands are usually low-to-medium molecular weight, which have short correlation times and no spin diffusion. Therefore they exhibit small positive NOEs. On the other hand, when a ligand is bound to a receptor (big protein), it acquires the motional properties of the macromolecule during the residence time in the bound state, thus exhibiting large correlation times $\tau_{c}$, extensive spin diffusion, and strong negative NOEs (i.e. transferred NOEs). Spin diffusion, which are typical for large molecules, is one of the major drawbacks of this experiment. In order to minimize the diffusion effect, short mixing-times should be used. In addition, NOE buildup curves, in which the NOE intensity of cross-peaks is recorded as a function of mixing time, might provide evidence for spin diffusion (Williamson 2009).

The tr-NOE cross peaks are finally treated as a normal NOE to derive distances restraints for later usage in structure determination.

Tr-NOESY experiments for Tau peptides in the presence of F-actin were performed at $278 \mathrm{~K}$, on Bruker 800 and $900 \mathrm{MHz}$ spectrometers equipped with cryogenic probeheads. Samples were prepared in NMR buffer with a peptide concentration of $800 \mu \mathrm{M}$ and $26.6 \mu \mathrm{M}$ of F-actin in a 30:1 ratio. 2D tr-NOESY experiments were carried out with 64 transients and a time domain of 2040 data points for 512 increments. A sweep width of 12 x 12 ppm was set in both dimensions. A total of five different mixing times (50, 80, 100, 150 and $250 \mathrm{~ms}$ ) were recorded, in order to determine a NOE buildup curve. The data were zero filled to 4096 x 1024 data points prior to Fourier transformation. Data were processed using Topspin 3.5pl5 and NMRPipe (Delaglio, Grzesiek et al. 1995), and analyzed using Spark 3.114 (T. D. Goddard and D. G. Kneller, http://www.cgl.ucsf.edu/home/sparky) . 


\subsubsection{Structure Calculation.}

Distance restraints were obtained from tr-NOESY contacts observed in $2 \mathrm{D}{ }^{1} \mathrm{H}-{ }^{1} \mathrm{H}$ NOESY spectra acquired with $100 \mathrm{~ms}$ mixing time. A total of 200 conformers were calculated using the standard simulated annealing schedule with 10000 torsion angle dynamics steps per conformer using CYANA 3.97 (Rodriguez, Schaefer et al. 2003, Güntert and Buchner 2015). The intensities of manually assigned cross-peaks were calibrated using the automated calibration method "calibrate", which is available for CYANA. This procedure converts NOESY cross-peak intensities into upper distance limits (UPL). There exists a functional relationship between the NOE peak intensity/ volume of NMR signal and the inter-proton distance, which is inversely proportional to the $6^{\text {th }}$ power of the distance between protons. This can be mathematically expressed as:

$$
V=\frac{A}{d^{6}}
$$

, where $V$ is the peak volume (or intensity), $d$ is the upper distance limit and $A$ is a calibration constant. The script (Fortran format) used for CYANA calculation is presented in Table 2.2.

All structures and distance restraints derived from CYANA were transformed to the XPLOR$\mathrm{NIH}$ input file format. Structures calculated by CYANA were subsequently refined in XPLORNIH using a restrained simulated annealing (SA) protocol (Schwieters et al., 2003). During simulated annealing, the temperature was lowered from $500 \mathrm{~K}$ down to $100 \mathrm{~K}$. To avoid potential miscalibration of NOE intensities, intra-residual NOEs were not considered. Lower and upper limits for short- and medium-range NOEs were set to $1.8 \AA$ and $6.0 \AA$, respectively. Statistical knowledge-based potentials for 2D and 3D torsion angle correlations were used and their force constants were ramped during the refinement from 0.002 to 10 . A total of 400 structures were calculated and the 20 lowest-energy conformers were selected for analysis using the protein structure validation software suite (PSVS) (Bhattacharya, Tejero et al. 2007). Visualization was performed using PYMOL (The PyMOL Molecular Graphics System, Version 1.5.0.4 Schrödinger, LLC) and MOLMOL (Koradi, Billeter et al. 1996) 
Table 2.2. Script used for structure calculation with a simulated annealing algorithm.

The script calculates 200 structures with 10000 annealing steps.

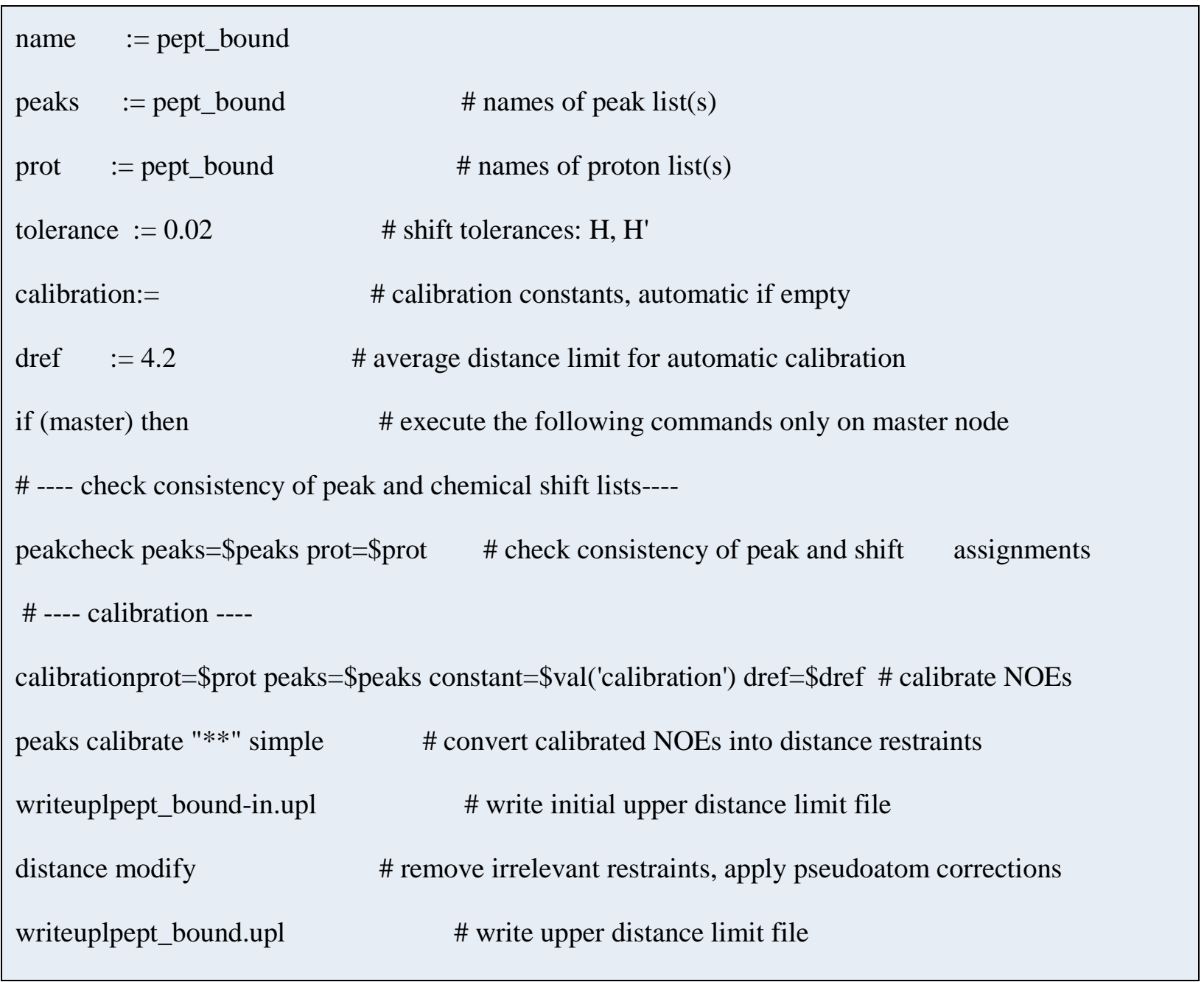

\subsection{Protein-protein docking}

Molecular docking can be used to model the interaction between a small molecule and a protein (McConkey BJ 2002). In principle docking can be achieved through two interrelated steps: first the conformations of the ligand in the active site are sampled, followed by ranking these conformations via a scoring function. Ideally, sampling algorithms should be able to reproduce the experimental binding mode and the scoring function should also rank it highest among all generated conformations. Docking efficiency will increase if binding sites are known in advance. 
The 3D structure of the peptide Tau(254-290) and knowledge about its binding site in F-actin were used to dock Tau(254-290) to F-actin. Molecular docking was performed using the Haddock web server (van Zundert, Rodrigues et al. 2016). Here, the cofilin-decorated F-actin structure (PDB id 3J0S (Galkin, Orlova et al. 2011)) was modified to use only the F-actin structure. Furthermore, we restricted the binding area to the hydrophobic pocket of F-actin between two actin protomers, which is exposed to the solvent (Dominguez 2004). Two consecutive actin protomers were used in the calculations. Residues V45-G48 and Q49 form protomer "I" and residues L140, Y143, A144, G146, R147, E167, G168, G342, L346, S348, T351, Q354, M355 and V370-F375 from protomer "II” were defined as active residues, while the surrounding residues were defined as passive residues. HADDOCK clustered 192 structures in 4 clusters, which represent $96.0 \%$ of the water-refined models generated by HADDOCK. Conformations from the cluster with the highest HADDOCK-score were used for further analysis. 


\section{Results}

\subsection{Interaction of Tau with G-actin}

The interaction between G-actin and Tau or Tau fragments was tested in two different ways. First the change in intensity of NBD-labeled actin in presence of increasing concentrations of ligands was analyzed and binding affinities were derived. Secondly, NMR-based experiments identified the Tau regions involved in the interaction with G-actin. In addition, Paramagnetic Relaxation Enhancement (PRE) as well as competition experiments provided information about the region through which G-actin recognizes Tau.

\subsubsection{Tau and Tau fragments bind to G-actin}

To determine of the binding affinity between G-actin and Tau or Tau fragments, the change in fluorescence intensity of $0.2 \mu \mathrm{M}$ NBD-labeled G-actin in presence of increasing concentrations of ligand was registered. Prior to the experiment, LatB was added to the actin solution to prevent filament formation. As shown in Figure 3.1a and Figure 3.1b the mixture of Tau or K18 with NBD-labeled G-actin does not change the position of the peak even though the binding place is saturated. Moreover, addition of K18 decreased the fluorescence intensity in a $67 \%$ while Tau does it in a 45\%. Affinities of Tau(254-284) or its phosphorylated version Tau(254-284pS262) bound to G-actin were also calculated Figure 3.1. For better analyze the results the calculated affinities for full-length Tau (hTau40), K18, Tau(254-284) and Tau(254-284pS262) are presented as a histogram Figure 3.1c. Error bars represent the standard deviations of three different experiments. The experiments reveal that either hTau40 or Tau fragments bind to Gactin with a high affinity. However, phosphorylation of Tau(254-284) on S262 decreased the affinity for binding to G-actin in one order of magnitude. 


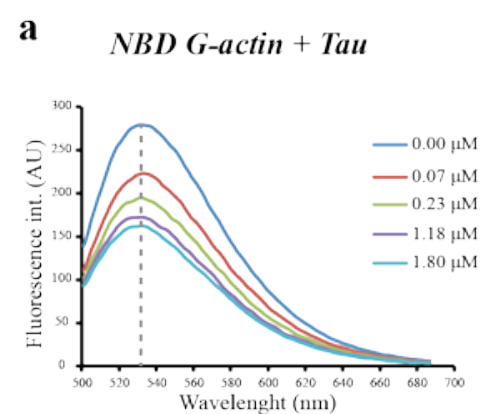

b

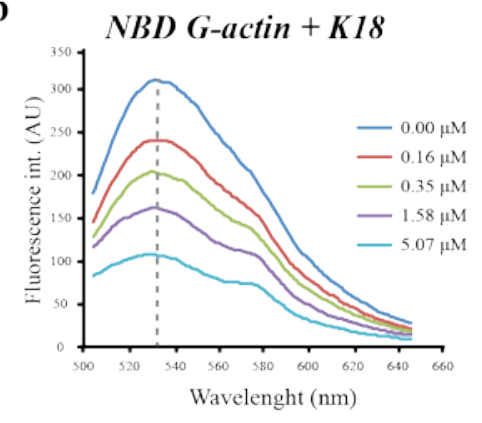

c

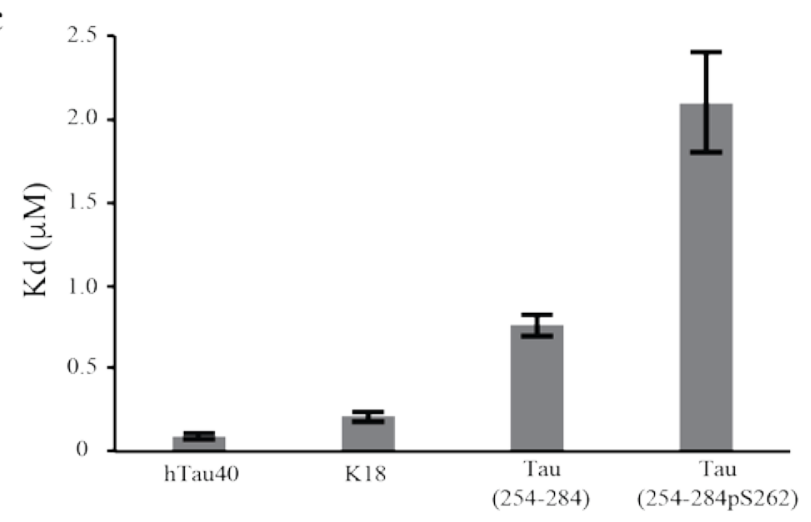

d

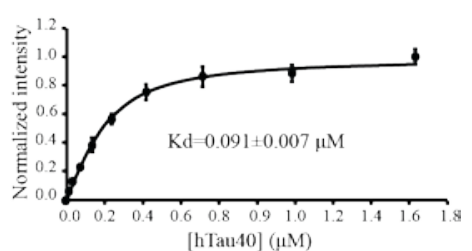

e

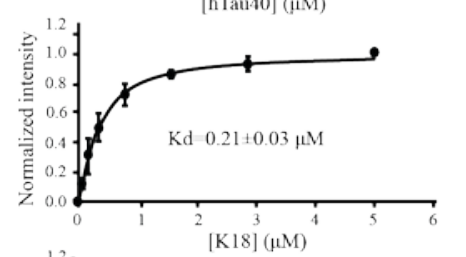

f

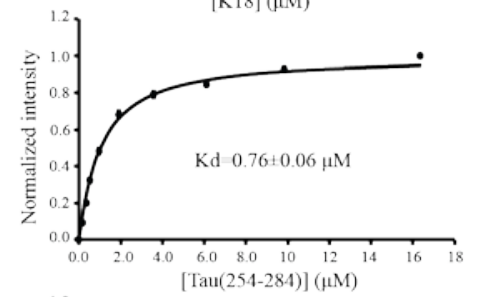

g

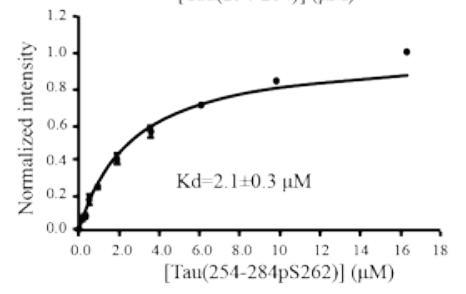

Figure 3.1. Fluorescence-based analysis of NBD-labeled G-actin bound to Tau and Tau constructs.

The superposition of fluorescence emission spectra of NBD-labeled G-actin in presence of incrising concentrations of hTau40 or K18 is presented in (a) and (b) respectively. A dashed line labels the maximum of emission for both cases at $530 \mathrm{~nm}$. (c) The dissociation constants for all the cases are plotted as a bar diagrams where error bars represent the standard deviation for three different experiments. The dissociation constants of hTau40 (d), K18 (e), Tau(254-284) (f) and Tau(254-284pS262) (g) were determined by the fitting of the experimental data to a single binding curve as explained in Materials and Methods section.

\subsubsection{Identification of binding regions of Tau in complex with G-actin}

The interaction between Tau and G-actin was probed by NMR spectroscopy to get insight into the regions of Tau that interact with actin. The analysis of the intensity peaks on a ${ }^{1} \mathrm{H}^{-15} \mathrm{~N}$ HSQC experiment from ${ }^{15} \mathrm{~N}$-labeled hTau40 in absence or presence of LatB-treated G-actin provided a rally powerful way to distinguish the Tau binding hot spots. In this experiment $30 \mu \mathrm{M}$ of $\mathrm{G}$-actin were mixed with $300 \mu \mathrm{M}$ LatB. After 30 minutes $15 \mu \mathrm{M}$ of ${ }^{15} \mathrm{~N}$-labeled hTau40 were added to the solution in a $1: 2$ ratio and a ${ }^{1} \mathrm{H}_{-}{ }^{15} \mathrm{~N}$ HSQC was recorded at $278 \mathrm{~K}$. A second experiment was measured only with hTau40 supplemented with LatB to be used as reference. 
The superposition of ${ }^{1} \mathrm{H}_{-}{ }^{15} \mathrm{~N}$ HSQC spectra from hTau40 in absence (gray) and presence of Gactin (red) is ahown in Figure 3.2. The previous resonance assignment of hTau40 was used for the analysis (Mukrasch, Bibow et al. 2009). Residues with lower NMR signal intensity such as G365, G302 (top-right panel) and K311 or V309 (bottom-right panel) are those in close contact to G-actin. For better analyze the regions involved in binding an intensity plot was built for both free and bound Tau. NMR intensities were plotted against the number of residues (Figure 3.3).

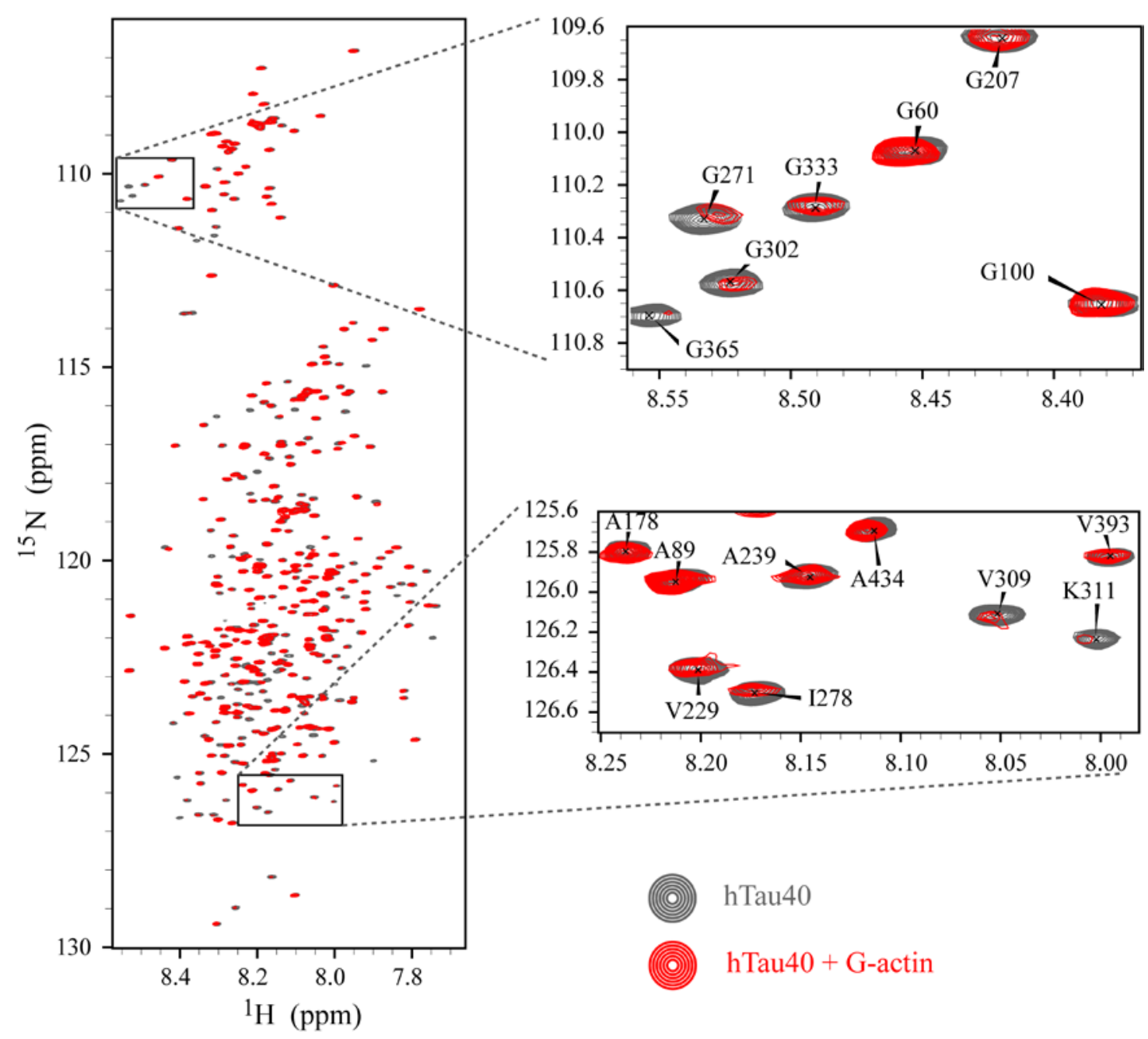

Figure 3.2. $2 \mathrm{D}{ }^{1} \mathrm{H}-{ }^{15} \mathrm{~N}$-HSQC spectra of hTau 40 in presence (red) and absence (grey) of G-actin.

At the right panel selected regions are magnified for better observe some of the residues involved in binding.

The non-uniform reduction in signal intensities in the spectrum of Tau bound to G-actin is caused by the exchange of Tau molecules between the free and bound states. The experiment revealed that the interacting regions of Tau in complex with G-actin comprise the proline-rich region (P1 and P2 top panel) and the repeat domain of hTau40 (R1-R4 top panel). Interestingly a 
very specific region between inserts N1 and N2 and proline-rich region (top panel) from residue L114-T123 that corresponds to a transient $\alpha$-helix on Tau (Mukrasch, Bibow et al. 2009) is also interacting with actin. According to the analysis the N-terminal part of Tau does not seems to be involved in binding to G-actin.

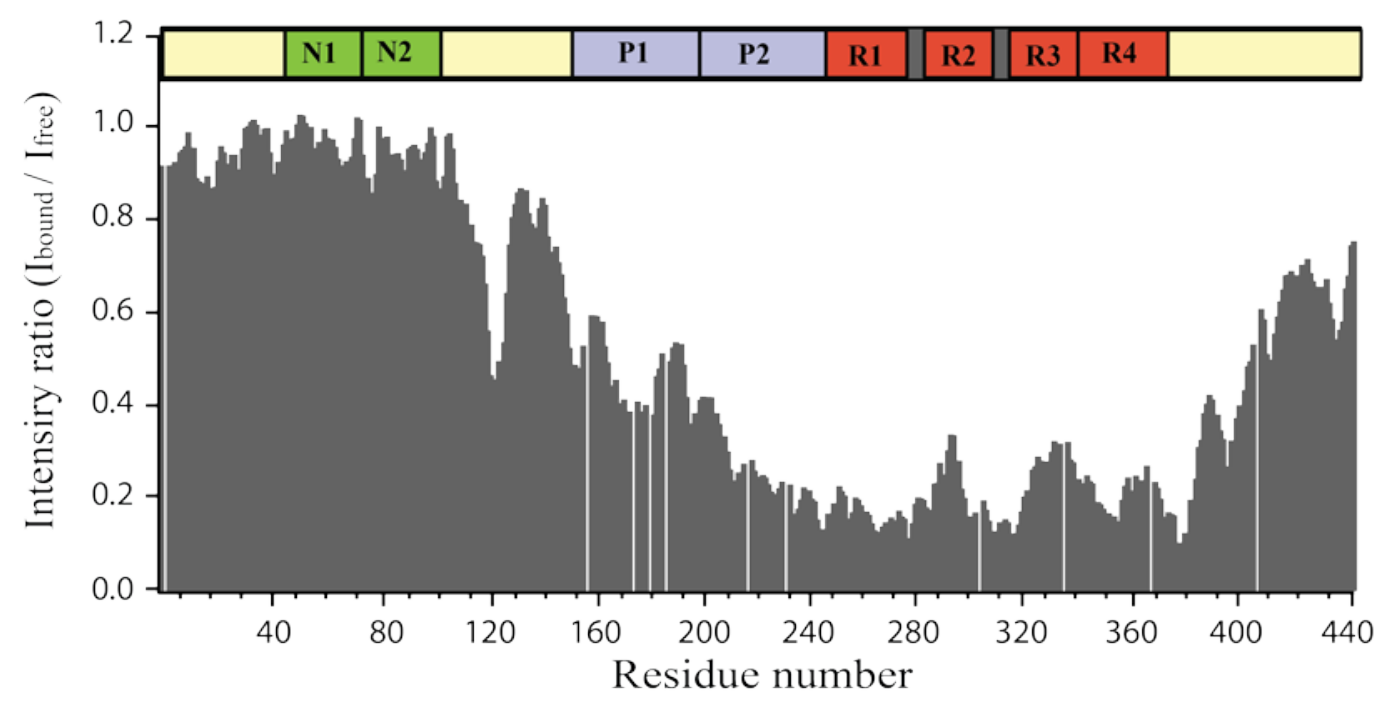

Figure 3.3. Tau/G-actin interaction.

NMR signal intensity ratio between signals observed in the ${ }^{1} \mathrm{H}-{ }^{15} \mathrm{~N}$ HSQC spectra for hTau40 in presence and absence of two-fold excess of G-actin. Organization of Tau domains is shown at the top panel: N1 and N2 represent the two inserts at the N-terminus, P1 and P2 the proline-rich regions and R1-R4 correspond to the different repeats.

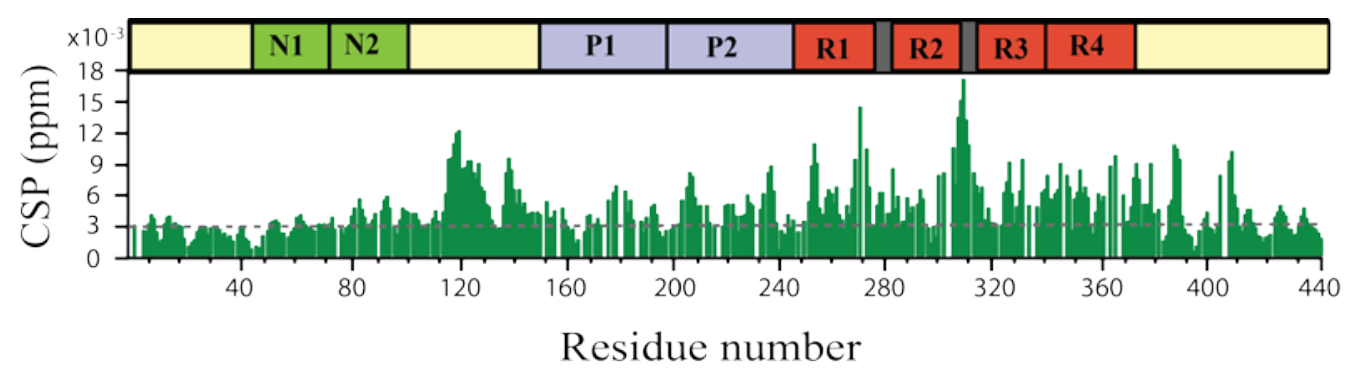

Figure 3.4. Combined chemical shift perturbation of Tau bound G-actin from ${ }^{1} \mathrm{H}^{15} \mathrm{~N}-\mathrm{HSQC}$.

Organization of Tau domains is shown at the top panel.

In addition the chemical shift perturbation (CSP) analysis revealed that the regions involved in binding are also showing perturbation in the chemical environment (Figure 3.4). Only the proline-rich and repeat regions show a binding effect as well as the region from L114-T123. 


\subsubsection{Tau fragments compete with Tau for binding to G-actin}

Upon identifying the Tau regions involved in binding competition experiments were performed between hTau40 and selected peptides from the identified binding hot spots. To this end, peptides Tau(211-242) form P2, Tau(254-290) from R1-R2 and Tau(292-319) from R2-R3 were synthesized.

The competition experiment between hTau40 and Tau(211-242) for binding to G-actin was performed in a titration-based HSQC experiment. To do that, a solution containing of $15 \mu \mathrm{M}$ of Tau and $30 \mu \mathrm{M}$ G-actin (1:2 ratio) was prepared in NMR buffer. All the samples were previously supplemented with 30-fold excess of LatB to prevent actin polymerization. Two different ${ }^{1} \mathrm{H}-{ }^{15} \mathrm{~N}$ HSQC experiments were acquired for Tau in absence and in presence of G-actin. Subsequently, the experiment was repeated twice upon adding 150 and $300 \mu \mathrm{M}$ of Tau(211-242). An intensity plot from the ${ }^{1} \mathrm{H}^{15}{ }^{15} \mathrm{HSQC}$ spectra was built (Figure 3.5a) and the ratio between the bound Tau ( $\left.\mathrm{I}_{\text {bound }}\right)$ and the free form $\left(\mathrm{I}_{\text {free }}\right)$ was compared. Interestingly, increasing amounts of peptide detaches the proline-rich and repeat regions of Tau from G-actin surface in a clear competitive manner.

Similarly a competition experiments were performed between hTau40 and the peptides Tau(254290) and Tau(292-319). The analysis of the intensity plots (Figure 3.5b and Figure 3.5c) showed a similar competition profile as in case of Tau(211-242).

The competition experiments suggest that all the peptides used in the analysis bind to the same place on G-actin and therefore addition of an excess of peptides detaches Tau from actin. 


\section{a}

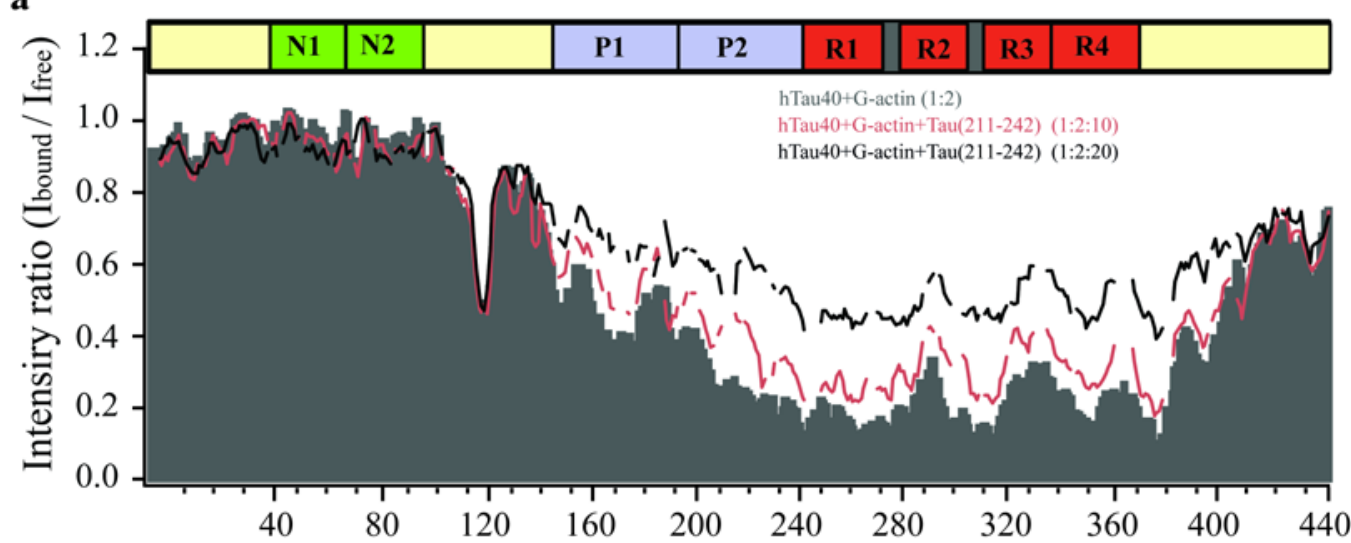

b

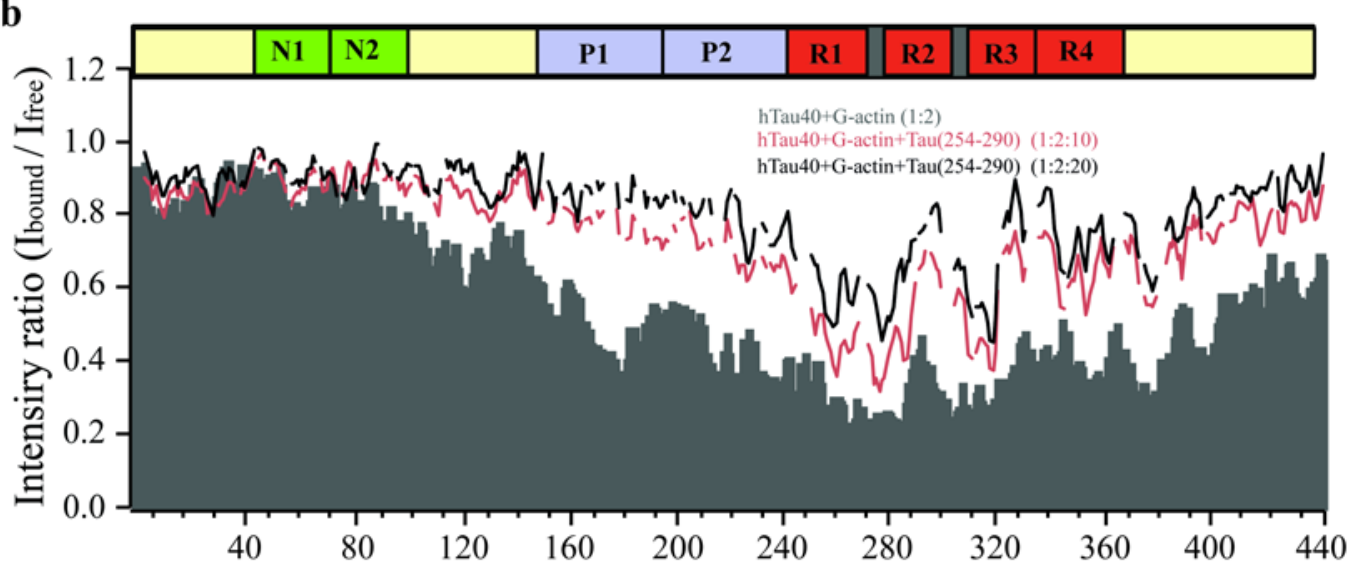

c

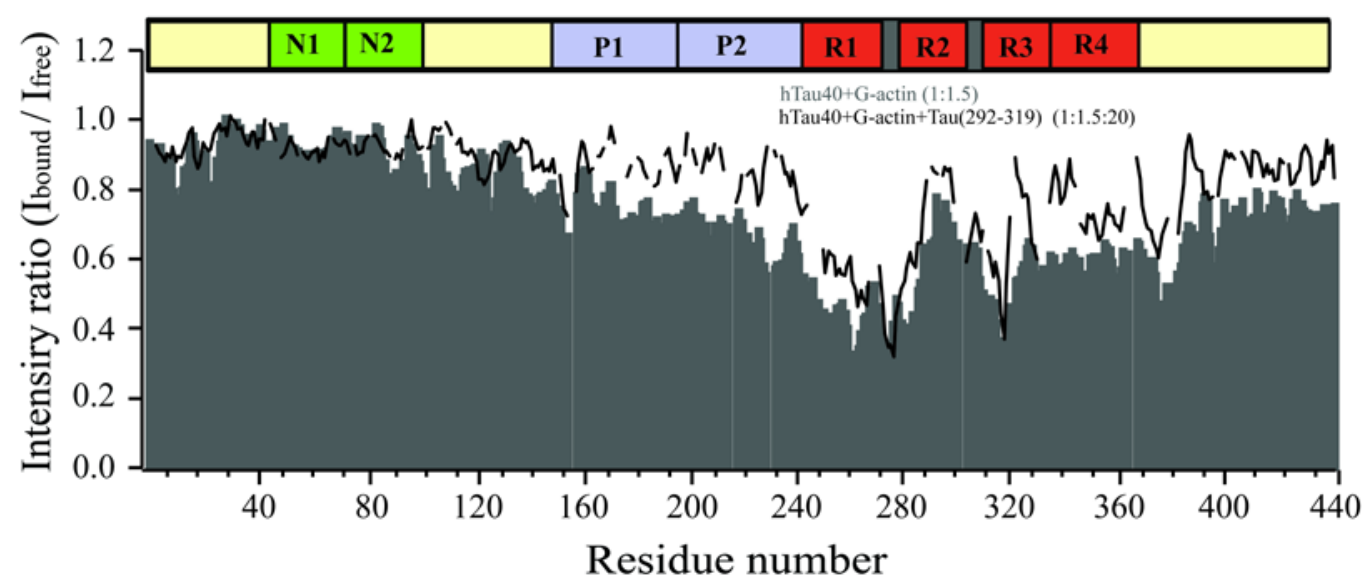

Figure 3.5. Competition experiments between Tau and Tau fragments for binding to G-actin.

(a) ${ }^{1} \mathrm{H}^{15} \mathrm{~N}$ HSQC intensity plots between Tau and Tau(211-242), Tau and Tau(254-290) (b), and Tau(292-319) (c). Organization of Tau domains is shown at the top of the three panels. 


\subsubsection{Mapping the binding of Tau on G-actin surface}

We have identified the regions of Tau that recognizes the G-actin molecule but where does Tau binds on G-actin surface?. To clarify this question competition experiment were performed with molecules with a known binding site on G-actin surface. These molecules are ciboulot (Cib) and the small molecule latrunculin B (LatB). Cib is a protein from the Thymosin- $\beta 4$ family, which binds to G-actin with a stoichiometry of $1: 1$ and a dissociation constants of $2.5 \pm 1 \mu \mathrm{M}$ (Boquet, Boujemaa et al. 2000). Cib binds to the hydrophobic pocket of G-actin between subdomains 1 and 3 (Dominguez 2004, Hertzog, van Heijenoort et al. 2004) as shown in Figure 3.7a. On the other hand LatB, derived from red sea sponges, binds to G-actin at the nucleotide-binding pocket between subdomains 2 and 4 (Figure 3.6a) with a stoichiometry of 1:1 avoiding the actin polymerization (Sasse, Kunze et al. 1998).

\subsubsection{Tau does not bind on the nucleotide-binding pocket of G-actin}
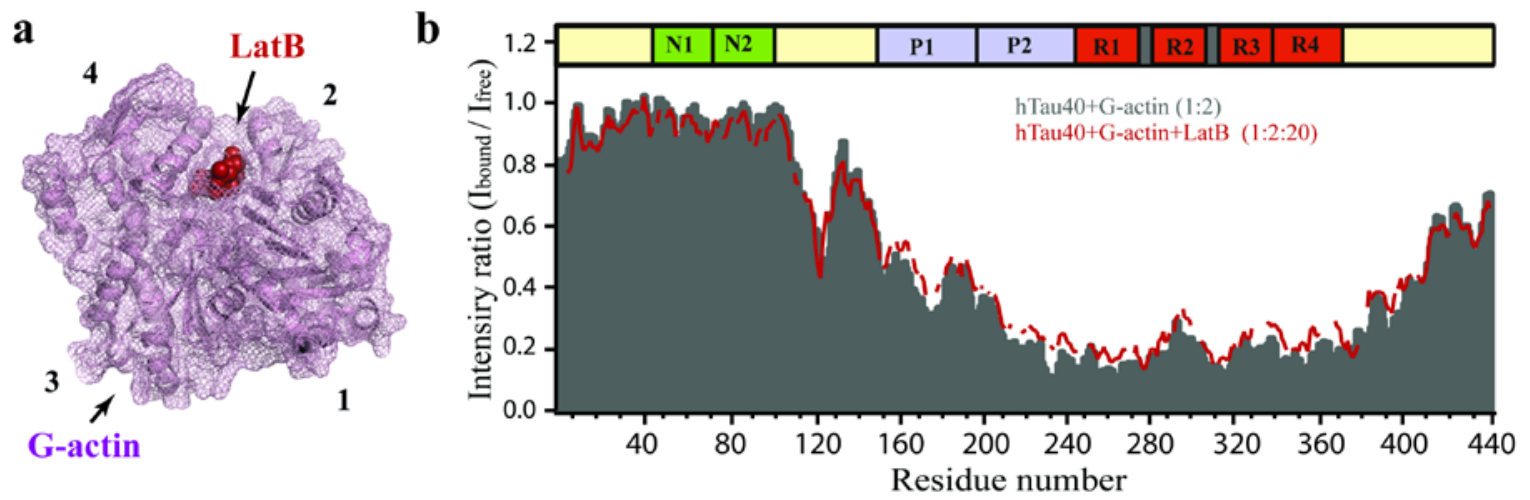

Figure 3.6. Tau does not compete with LatB for binding to G-actin on the nucleotide-binding pocket.

Competition experiment between Tau and LatB for binding to G-actin. (a) Cartoon representation of Gactin (pink) in complex with LatB (red) (PDB id 2Q0U (Allingham, Miles et al. 2007)). The four subdomains of G-actin are numbered. (b) Intensity plots derived from the ${ }^{1} \mathrm{H}-{ }^{15} \mathrm{~N}-\mathrm{HSQC}$ experiments where the intensity ratio between Tau bound to G-actin $\left(\mathrm{I}_{\text {bound }}\right)$ and in solution $\left(\mathrm{I}_{\text {free }}\right)$ is plotted against the number of residues (grey). The binding profile in presence of LatB is shown in red. Organization of Tau domains is shown at the top panel.

To determine whether Tau binds to the nucleotide binding of G-actin a competition experiment between hTau40 and LatB was performed. In this experiment the intensities of the ${ }^{1} \mathrm{H}^{-}{ }^{15} \mathrm{~N}$ HSQC spectrum when LatB is added to the Tau/G-actin complex for both the reference experiment (Tau 
alone) and the complex (Tau/G-actin) were compared. Here, 20-fold excess of LatB was added to the complex in a 1:2:20 ratio and supplemented with $2 \%$ DMSO for solubilize the molecule.

The intensity plot from the titration is shown in Figure 3.6b. The experiment revealed that there is no competition between Tau and LatB at this selected ratio meaning that Tau does not bind on the nucleotide-binding pocket of G-actin.

\subsubsection{Tau binds to the hydrophobic pocket of G-actin}

\subsection{Competition experiment with $\operatorname{Cib(D1)}$}

Similarly a competition experiment was performed with hTau40 and Cib(D1). Cib(D1) is the binding region of ciboulot that binds to G-actin and comprises the first 31 residues from the Nterminal part of the full-length protein. The 3D structure of Cib(D1) in complex with G-actin is shown in Figure 3.7a. Previously before adding Cib(D1) the G-actin was incubated with 10-fold excess of LatB to prevent actin assembly. We discard any interaction between Cib and LatB because they have a different binding site on actin (Guo, Shillcock et al. 2010, Dominguez and Holmes 2011). As in case of LatB, a single-point titration experiment was performed where 20fold excess of Cib(D1) was added to the Tau/G-actin complex. For better analyze the experimental data from the ${ }^{1} \mathrm{H}_{-}{ }^{15} \mathrm{~N}$ HSQC spectra, the Tau sequence was divided in three different regions: Projection domain (PD), Proline-rich region (PR) and the repeat region (RR) as shown in Figure 3.7b. A bar diagram was constructed where the average intensities of the Tau/Gactin complex in absence (grey bars) and in presence of 20-fold excess of ciboulot (blue bars) were compared Figure 3.7c. The interaction of Tau with monomeric actin decreased the average intensities of the PR and the RR but the addition of excess of ciboulot to the complex detached Tau from actin surface in a clear competitive manner.

The competitions between $\mathrm{Cib}$ and Tau constructs were also tested using STD-NMR spectroscopy. The 1D STD-NMR method was used by saturating resonances on G-actin at -1 ppm corresponding to the methyl proton region on G-actin. As a reference, a second 1D experiment was recorded by saturating the sample at $60 \mathrm{ppm}$, which is far from any proton resonance in the sample. The saturation of methyl protons in G-actin is first spread via spin diffusion to all G-actin protons. Subsequently the magnetization is transferred to the protons of the ligand via dipolar coupling whose transiently bind to G-actin. In this way, weak interactions between ligand and protein are detected and protons involved in the binding are identified. 
a

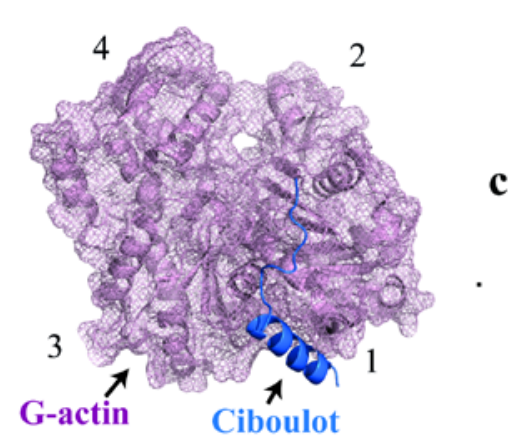

b Projection domain Proline-rich region Repeat region

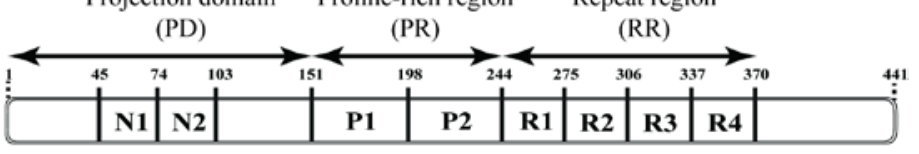

c

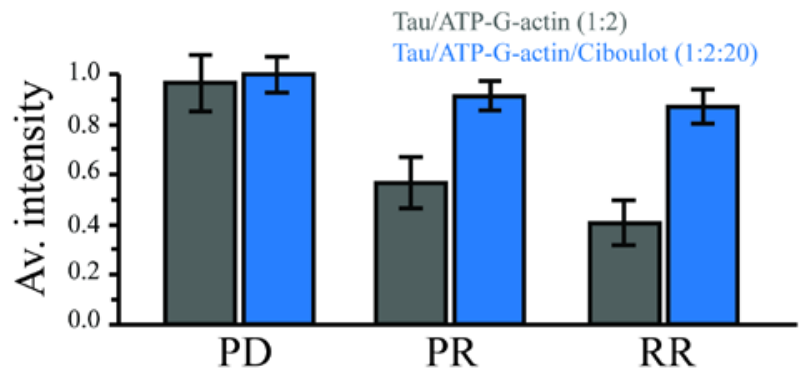

Figure 3.7. Tau and ciboulot shares the binding site on G-actin surface.

(a) Cartoon representation of G-actin (pink) in complex with Cib(D1) (blue light) from protein databank (PDB id 1SQK (Hertzog, van Heijenoort et al. 2004)). Actin molecule is subdivided on subdomains 1-4. (b) Domain distribution of Tau where three important regions are gihlighted as Projection domain (PD), Proline-rich region (PR) and Repeat region (RR). (c) The ${ }^{1} \mathrm{H}-{ }^{15} \mathrm{~N}$ HSQC average intensity of the different regions of Tau represented in (b) are shown as a bar diagram were intensities of Tau in presence of Gactin (grey bars) are compared to the intensities from ternary compex in presence of 20-fold excess of ciboulot (blue bars).

For these experiments increasing concentrations of $\mathrm{Cib}(\mathrm{D} 1)$ were added to a mixture containing G-actin and 60-fold excess of Tau peptides. The analysis of the STD spectrum revealed that some of the peaks form the peptide Tau(254-290) (see grey arrow on Figure 3.8a) strongly decreased with the addition of Cib(D1). This means that Cib(D1) competes with this peptide for binding to G-actin, a finding that correlates with the observation in Figure 3.7b. Other regions of the spectrum cannot be analyzed because of the overlap with the signals coming from Cib(D1). If the same experiment is performed but in presence of two peptides from P1 and P2 regions such as Tau(161-188) and $\operatorname{Tau}(211-242)$ respectively, the analysis is not feasible because of the overlap between signals form peptides and Cib (Figure 3.8b and Figure 3.8c). Nevertheless competition between Cib and these two Tau peptides cannot be discarded. 
a
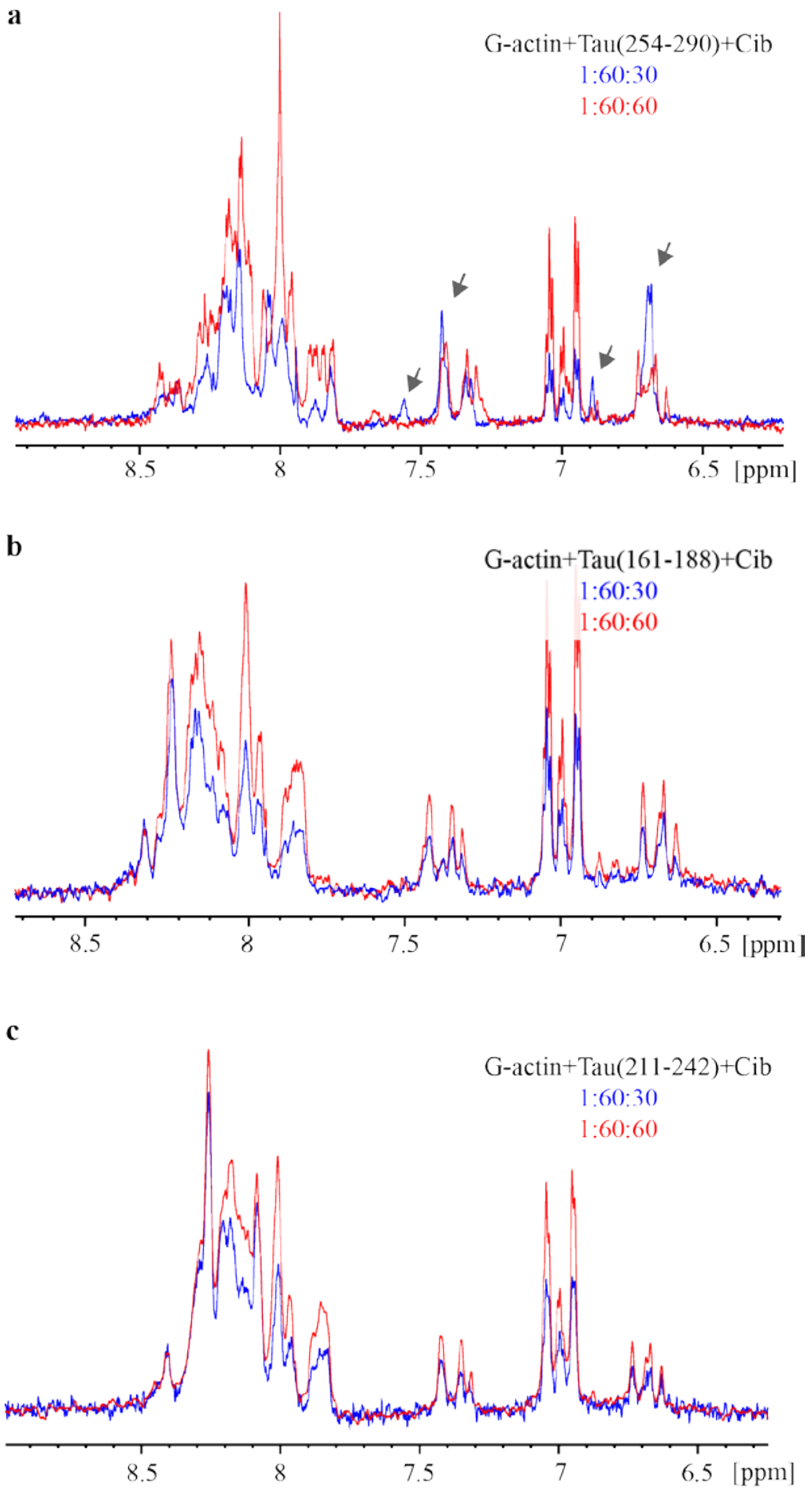

Figure 3.8. STD-based competition experiment between Cib and Tau fragments for binding to Gactin.

(a) Competition experiment between $\mathrm{Tau}(254-290)$ and $\mathrm{Cib}$ for binding to G-actin. Gray arrows label residues from the peptide showing a competition effect upon adding Cib. (b) Competition experiment between Tau(161-188) and Cib for binding to G-actin. (c) Competition experiment between Tau(211-242) and Cib for binding to G-actin. 


\subsection{Further evidences form the binding of Tau on the hydrophobic pocket of G- actin}

To further analyze whether Tau binds on the hydrophobic pocket of G-actin we used the effect of paramagnetic centers on NMR resonances. Particularly useful are paramagnetic nitroxide tags such as MTSL, which can be attached to cysteine residues in proteins. The attachment of MTSL to the protein causes an enhanced relaxation of protons, which are less than $\sim 25 \AA$ from the paramagnetic center (Gillespie and Shortle 1997). We thus sought a way to attach MTSL to a single cysteine in actin. Actin in total contains six cysteine residues. However, only the Cterminal C374 is exposed to the solvent (Figure 3.9a) (Yasunaga and Wakabayashi 2001). Tau was then mixed with two-fold excess of MTSL-labeled G-actin in a 1:2 ratio (G-actin in excess). The ${ }^{1} \mathrm{H}^{-15} \mathrm{~N}$ HSQC spectra of both paramagnetic and diamagnetic states of actin were acquired. The superimposed spectra in absence (red) and presence (black) of the paramagnetic tag are shown in Figure 3.9b.

Subsequently a single residue analysis was performed taking the ratio between the paramagnetic ( $\mathrm{I}_{\text {para }}$ ) and diamagnetic ( $\mathrm{I}_{\text {dia }}$ ) contributions as represented by the black line on Figure 3.9c). When diamagnetic and paramagnetic contributions are compared, residues in close contact with MTSL experience an enhanced relaxation because of the dipolar interaction. The PRE profile contained eight peaks corresponding to residues L243-K280, N286-K298, G301-K311, V318-G333 and Q351-T361. Interestingly these regions comprise the four KXGS motifs of Tau (red arrows) as well as the two hexapeptides ${ }^{275} V_{\text {QIINK }}{ }^{280}$ and ${ }^{306} \mathrm{VQIYK}^{311}$ (green arrows) (Figure 3.9c,d), which are related to Tau aggregation in Alzheimer’s disease (von Bergen, Friedhoff et al. 2000). 

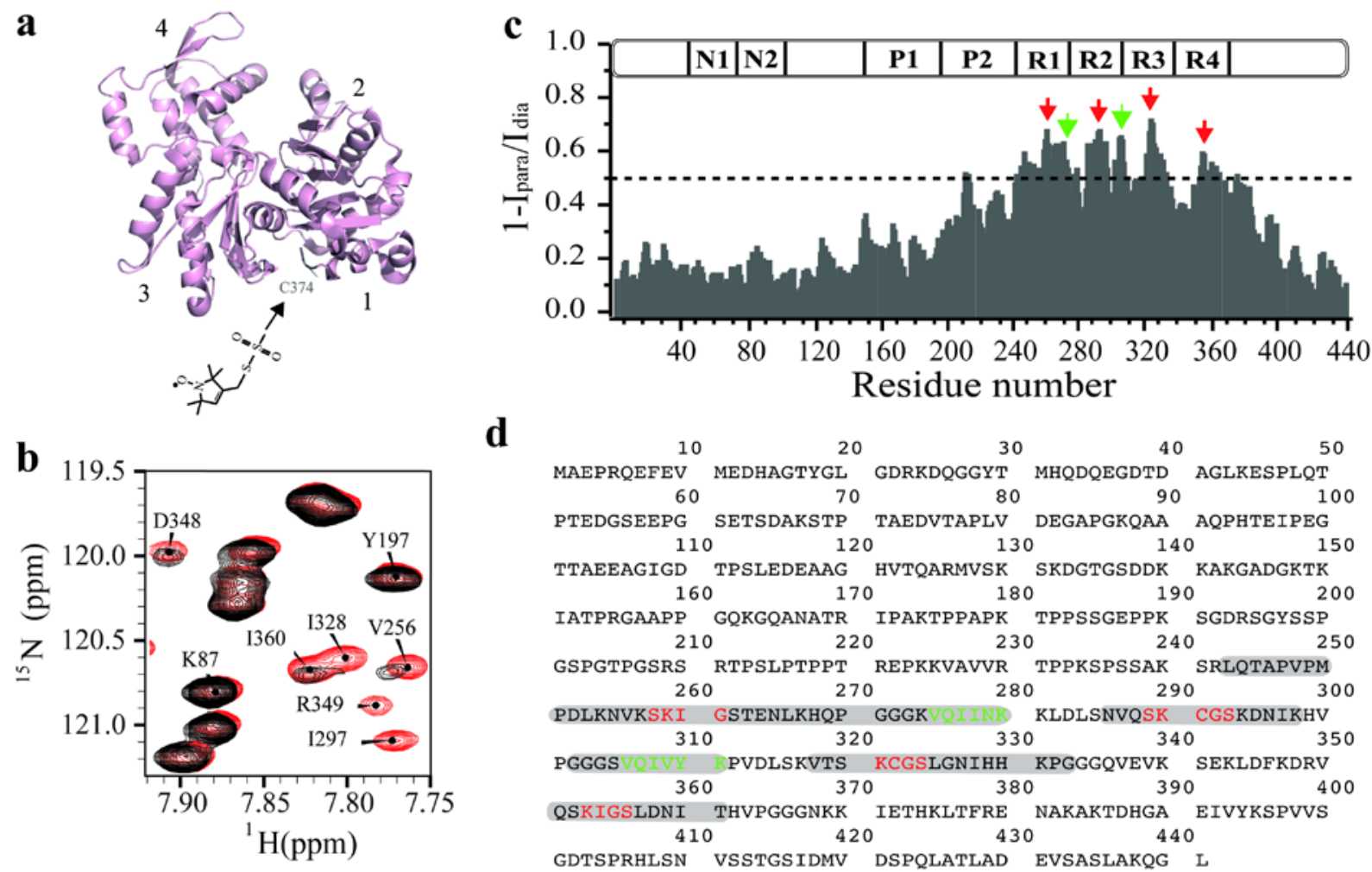

Figure 3.9. The PRE approach reveals that Tau binds nearby the hydrophobic pocket of G-Actin.

(a), MTSL tagging on C374 from native G-actin (pink) (PDB id 3HBT (Wang, Robinson et al. 2010)) where diferent subdomains of actin are numbered. (b) Superimposed ${ }^{1} \mathrm{H}-{ }^{15} \mathrm{~N}-\mathrm{HSQC}$ spectra in presence (black) and absence (red) of MTSL are presented while peaks from some residues are labeled. (c), The plot represents the intensity ratio between a sample containing Tau and MTSL-labeled G-actin ( $\left.\mathrm{I}_{\text {para }}\right)$ and its diamagnetic version $\left(\mathrm{I}_{\mathrm{dia}}\right)$. Red arrows highlight those regions where KXGS motifs are present while the green ones correspond to the two hexapeptides of Tau. Organization of tau domains is shown at the top panel. (d) Amino acid sequence of full-length Tau. Grey bars comprise the most afected residues above the dashed line in (c). KXGS morifs are colored in red while hexapeptides are in green.

\subsection{Interaction of Tau with F-actin}

Several authors have reported the interaction between Tau and F-actin by using different in vivo and in vitro assays (Biernat and Mandelkow 1999, Yu and Rasenick 2006, Spears, Furgerson et al. 2014). Tau was found to interact with F-actin in Drosophila neurons promoting the F-actin bundle formation (Fulga, Elson-Schwab et al. 2007) where the presence of Tau increased the Factin stability while G-actin levels remained unaffected. In the same study an in vitro assay showed that Tau enhance the F-actin stability even in presence of swinholide-A, an F-actin 
depolymerizing agent. Nevertheless the nature of this interaction as well as the interacting regions on Tau and F-actin are still unclear. Therefore we decide to study this interaction by using NMR as well as other biophysical techniques.

\subsubsection{Tau interacts with and promotes the bundles of actin filaments}

The interaction between Tau and F-actin was tested by means of EM. To do so, a $20 \mu$ l solution of equimolar ratio of F-actin and Tau were prepared in GAB. According to the EM pictures (Figure 3.10a), the addition of Tau to the F-actin solution promoted F-actin bundles (closed arrowheads). EM pictures revealed that the bundling was not $100 \%$ efficient and still some single filaments (open arrowheads) are observed in the background. The diameters of these filaments are in the range of $7 \mathrm{~nm}$ which in agreement with the literature (Egelman 1985, Egelman 2003). Bundles with diameters ranging between 20 and $150 \mathrm{~nm}$ are observed.

a

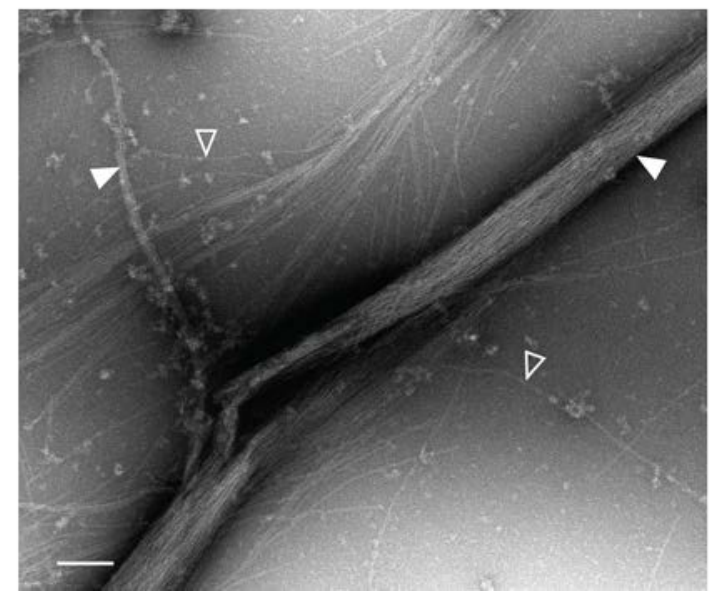

b

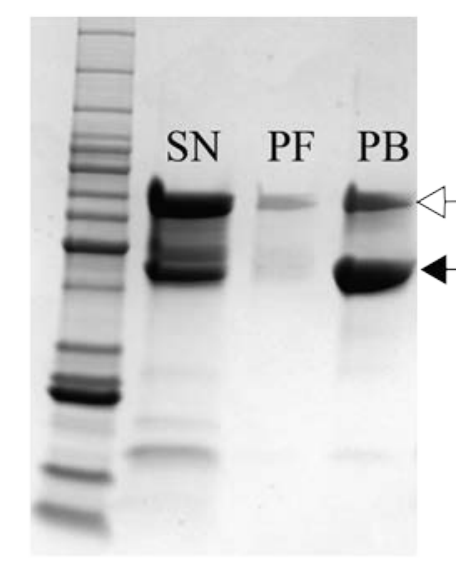

Figure 3.10. Negatively stained electron micrograph from Tau-promoted F-actin bundles.

(a), Tau interacts with F-actin and promotes bundles formation at low ionic strength as seen by electron microscopy. In the figure single filaments as well as F-actin bundles are labeled with open and closed arrowheads respectively. Magnification bar correspond to $150 \mathrm{~nm}$. (b) The interaction was also analyzed by SDS polyacrylamide gel. Here the previously treated samples by co-sedimentation assay were loaded in a 4-20\% gradient gel. A sample containing only Tau-promoted bundles of F-actin was loaded in lane PB while individual filaments were loaded on lane PF. The final supernatant containing only G-actin and Tau were loaded in lane SN. Open and closed arrowheads represent a molecular weight corresponding to Tau and actin respectively. 
Co-sedimentation assay provided further support for the Tau-promoted bundles. In this experiment the same sample was differentially centrifuged as explained in material and methods. Pellets were reconstituted in $80 \mu \mathrm{GAB}$ supplemented with $10 \mathrm{mM} \mathrm{NaCl}$ and denatured with Loading Buffer Blue (2X). Afterwards, aliquots form supernatant (SN), filament (PF) and bundles (PB) fractions were loaded in a 4-20\% gradient gel as shown in Figure 3.10b. According to the gel quantification a $63 \%$ of Tau and $49 \%$ actin remains in the supernatant meaning that the polymerization of actin was not $100 \%$ efficient. Only an $8 \%$ of Tau molecules are bound to a $3 \%$ of single filaments. This observation is in agreement with what we so by EM where still is possible to observe single filament at the background. On the other hand the other $29 \%$ of Tau appears to be cross-linking F-actin as seen in lane PB. These results confirm that Tau is able to interact with F-actin and promote the bundle formation, a finding that has been broadly boarded in literature.

\subsubsection{NBD-fluorescence reveals strong binding between Tau and F-actin}

In order to gain insight into the affinity of the Tau/F-actin-interaction, the change in fluorescence of 7-chloro-4-nitrobenz-2-oxa-1,3-diazole (NBD)-labeled F-actin upon addition of Tau was followed. $0.2 \mu \mathrm{M}$ of NBD-labeled and phalloidin-stabilized F-actin were mixed with increasing amounts of Tau. The addition of Tau up to saturation decreased the NBD-fluorescence in $85 \%$ as shown in Figure 3.11a almost twice that in presence of G-actin. The experiment was repeated three times and the errors are reported. A fit to the experimental data using a single binding site model resulted in a dissociation constant of $60 \pm 10 \mathrm{nM}$ (Figure 3.11b). Other stoichiometries, however, cannot be excluded. The derived dissociation constant is lower than a previously reported value of $241 \pm 43 \mathrm{nM}$ (Elie, Prezel et al. 2015). This is likely because of differences in ionic strength, because previous studies used phosphate buffer (Elie, Prezel et al. 2015), while we chose general actin buffer. 
a

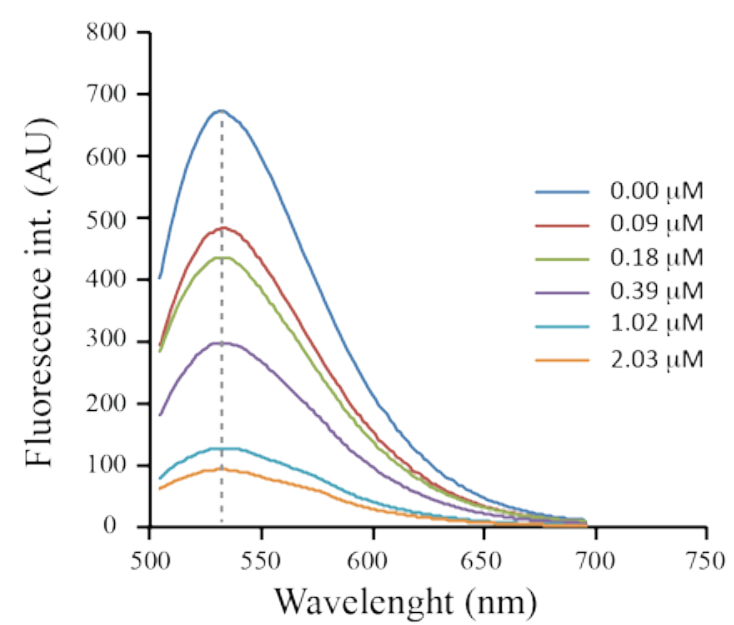

b

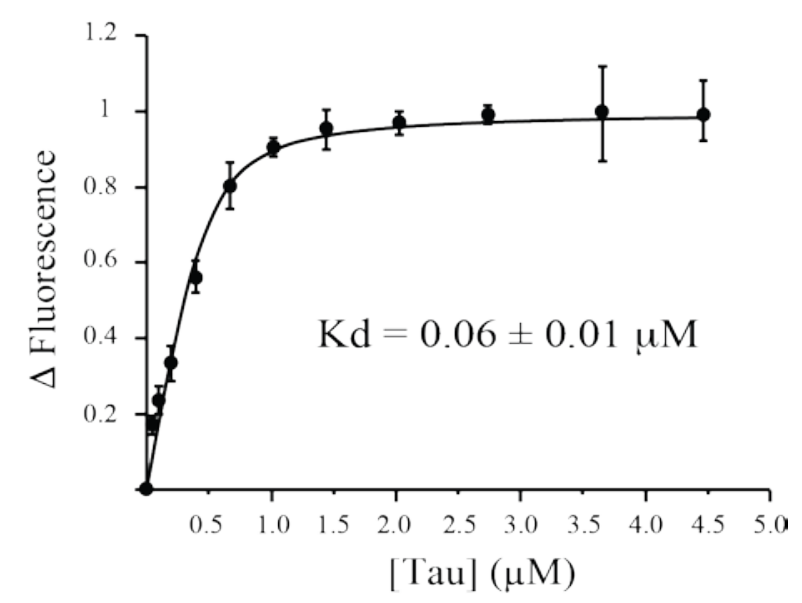

Figure 3.11. NBD-fluorescence reveals a strong binding between Tau and F-actin.

The superposition of the fluorescence emission spectra of NBD-labeled F-actin in presence of increasing concentrations of hTau40 is showed in (a). A dashed line represents the maximum of emission around $530 \mathrm{~nm}$. (b) The dissociation constant of hTau40 was determined by the fitting of the experimental data to a single binding curve as explained in Materials and Methods section.

\subsubsection{Identification of binding regions of Tau in complex with F-actin}

In order to identify residues in Tau that bind to F-actin, we used NMR spectroscopy. F-actin and ${ }^{15} \mathrm{~N}$-labeled Tau were mixed in a 2:1 molar ratio and a two-dimensional ${ }^{1} \mathrm{H}-{ }^{15} \mathrm{~N}$ HSQC experiment was recorded. Comparison with a reference spectrum of Tau alone showed that addition of F-actin into the Tau pool decreased the signal intensity of several Tau residues (Figure 3.12). Very little chemical shift changes were observed, indicating that the binding process is intermediate to slow on the NMR time scale. According to the resonance assignment of Tau (Mukrasch, Bibow et al. 2009), cross-peaks from residues such as S262, N265 and T319 were strongly attenuated. Other residues including T175 and T217 were less affected, and S46 and S64 were not perturbed at all. 


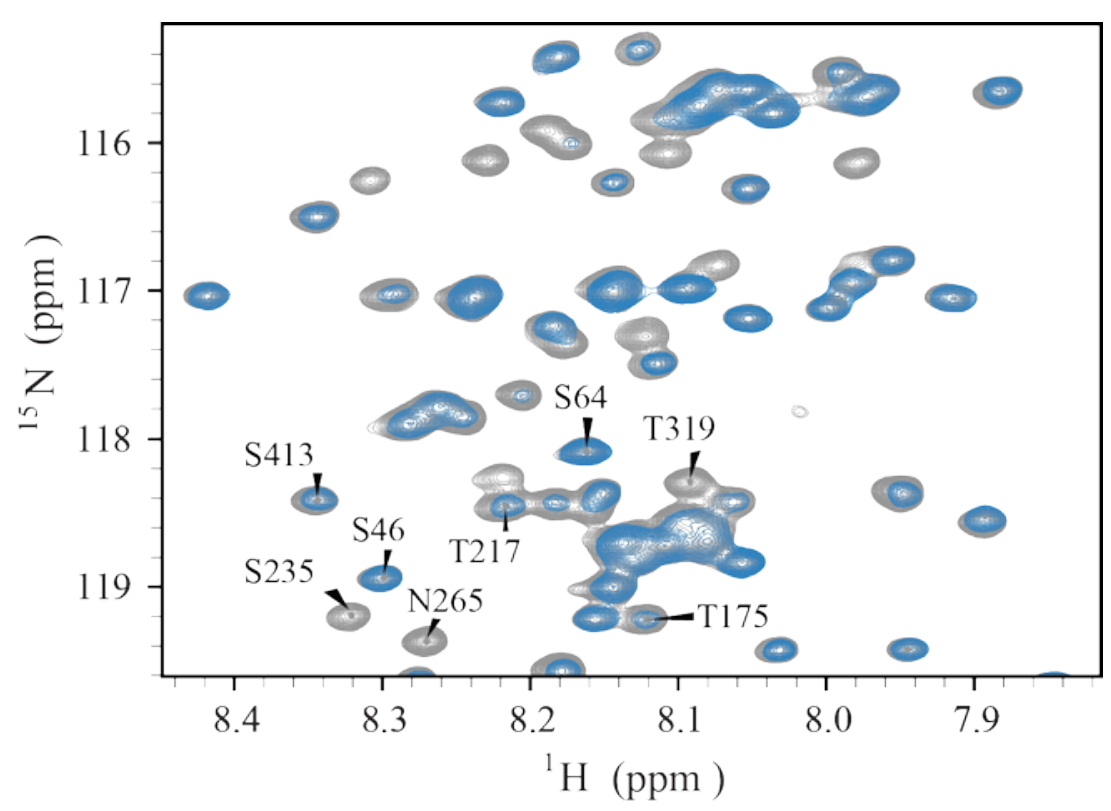

Figure 3.12. Superposition of ${ }^{1} \mathrm{H}^{-15} \mathrm{~N}$ HSQC spectra of hTau40 in absence (gray) and presence (blue) of F-actin.

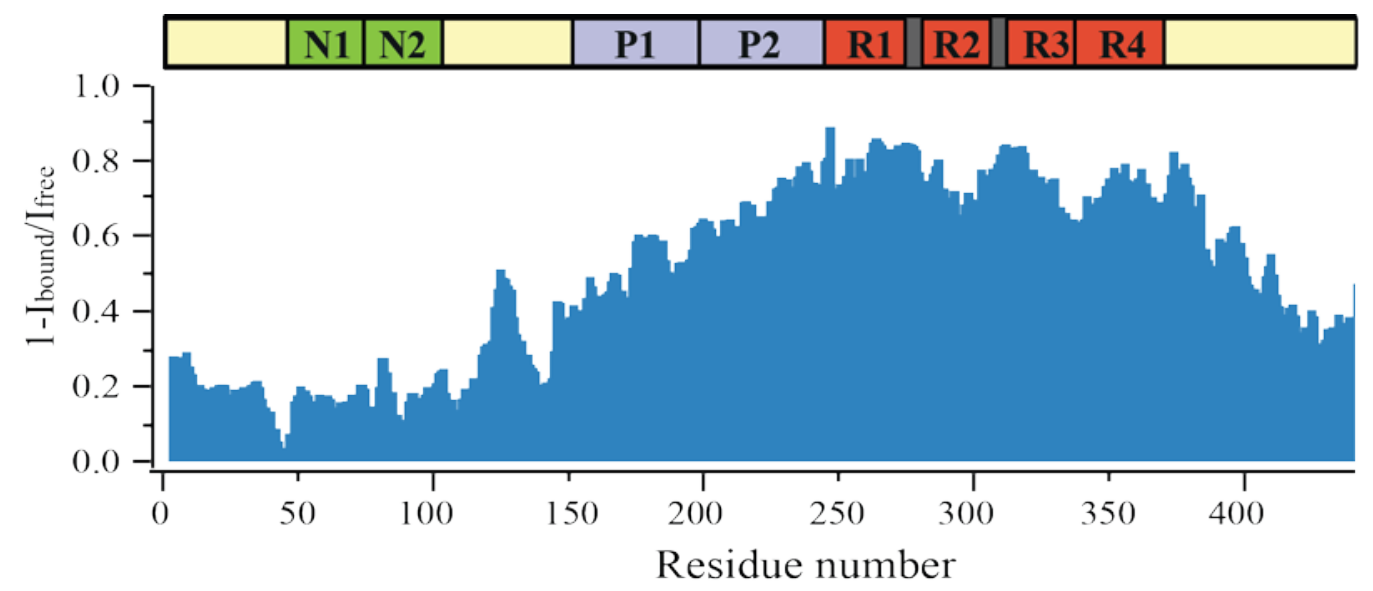

Figure 3.13. Residue-specific changes in ${ }^{1} \mathrm{H}-{ }^{15} \mathrm{~N}$ HSQC signal intensities of Tau upon addition of Factin.

$I_{\text {free }}$ and $I_{\text {bound }}$ are intensities in the absence and presence of a two-fold excess of F-actin. Organization of Tau domains is shown at the top panel.

Figure 3.13 highlights the sequence specific intensity ratio of all non-overlapping cross-peaks of Tau in the presence and absence of F-actin. Low (1-Ibound/Ifree) values are expected for residues that do not directly bind to F-actin. In contrast, residues, which are bound to F-actin, should have large (1-Ibound/Ifree) values, because their resonances would - due to the high molecular weight of the complex - no longer be observable by solution-state NMR. The analysis reveals that the proline-rich regions P1-P2 and the four pseudo-repeats contribute to the Tau/F-actin interaction. 
The strongest NMR signal attenuation was observed for K254-V287, K294-K331 and E342D358, suggesting that these Tau residues have the highest affinity towards F-actin.

\subsubsection{Short Tau fragments from the MBD also promote F-actin bundles formation}

To directly prove the effect of short regions of Tau on the binding a Tau fragment ranging from K254 to K290 (Tau(254-290)) was synthesized. Then $10 \mu \mathrm{M}$ of F-actin were incubated with a 10-fold excess of Tau(254-290) in GAB and taken to EM. Interestingly Tau(254-290) also promoted F-actin bundles (Figure 3.14a) but still some single filaments are remaining at the background. Unlike hTau40, the bundles are less populated having a maximum diameter of around $60 \mathrm{~nm}$. Similarly other Tau constructs located in the MBD like K18, which comprises the repeats from R1 to R4 and Tau(292-319), were also tested by EM. Both Tau fragments showed bundling effect on F-actin as well (Figure 3.14c and Figure 3.14b) although the effect is more dramatic in case of K18. However when a shorter peptide like Tau(254-268) is used no bundles effect is observed Figure 3.14d. This result is not surprising if we consider that the cross-linking effect of Tau is strongly dependent on the number of residues involved in binding as reported by Elie, Prezel et al. (Elie, Prezel et al. 2015).

The affinities of Tau peptides like K18, Tau(254-284) and its phosphorylated version were also tested following the change in intensity of NBD at low ionic stress. A decrease in fluorescence upon addition of partner was observed for all the cases. We determined that K18 binds to F-actin with an affinity of $110 \pm 1 \mathrm{nM}$ reducing the intensity of NBD in a $46 \%$. On the other hand $\operatorname{Tau}(254-284)$ binds to F-actin with a lower affinity $(0.630 \pm 0.08 \mu \mathrm{M})$ while the intensity decreases only a $30 \%$. Surprisingly, when this peptide is phosphorylated on S262 its affinity is a bit lower that the wild type but still remains in the same order of magnitude $(\mathrm{Kd}=0.95 \pm 0.04$ $\mu \mathrm{M})$. For better analyze the differences in affinities a bar diagram was made as shown in Figure 3.15 . 
a

F-actin + Tau(254-290)

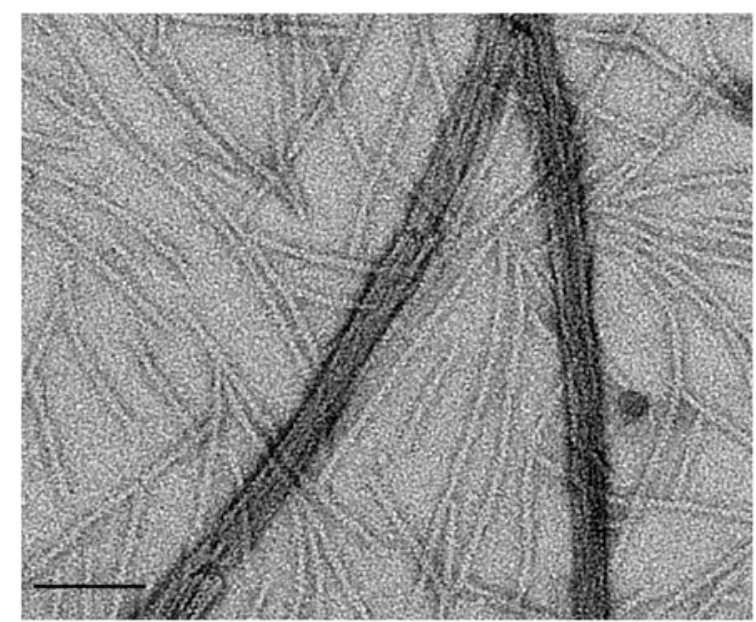

c

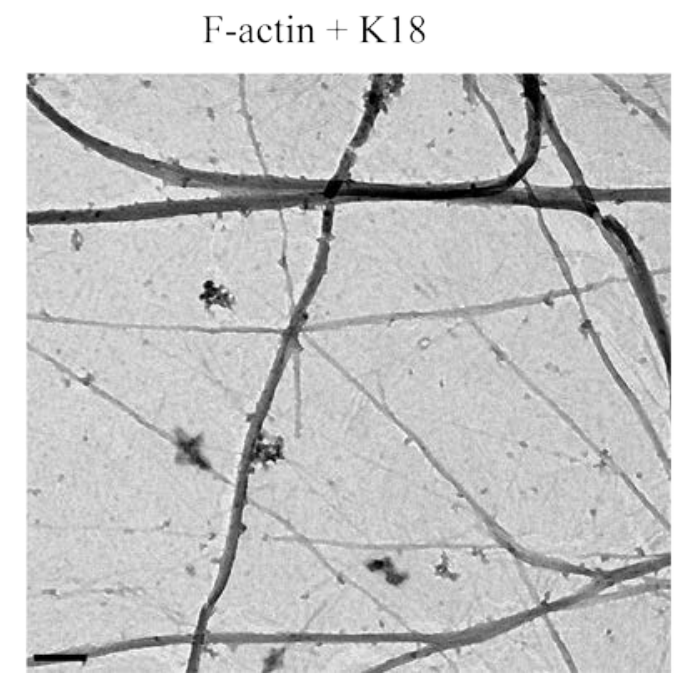

b F-actin + Tau(292-319)

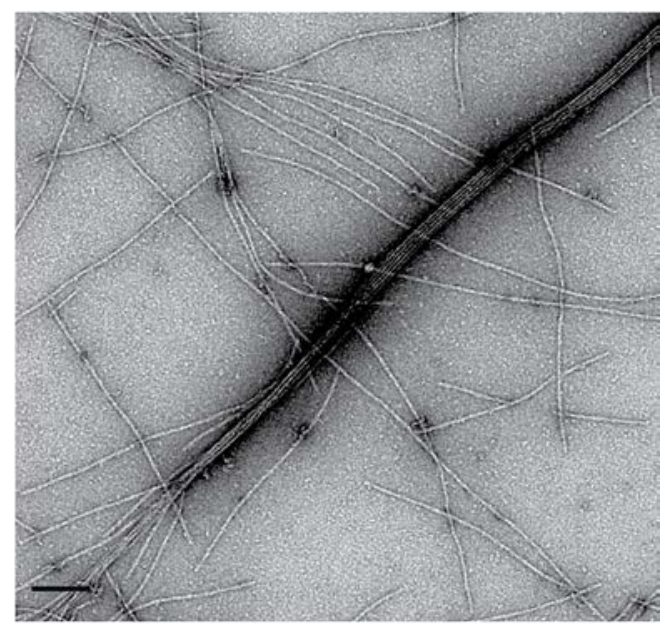

d

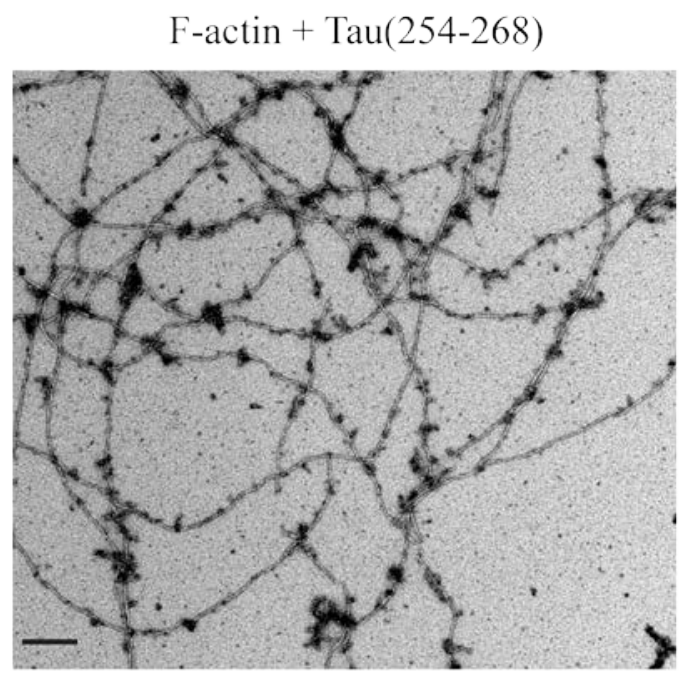

Figure 3.14. Electron micrographs from F-actin in complex with Tau peptides.

(a) 10-fold excess of $\mathrm{Tau}(254-290)$ promotes bundles of F-actin. A similar effect can be observed when Tau(292-319) is used (b). (c) A longer Tau construct comprising the MBD (K18) has even a more dramatic effect. However a 30-fold excess of a shorter construct like Tau(254-268) does not promote the bundle formation (d). Magnification bars correspond to $150 \mathrm{~nm}$ in all the pictures. 


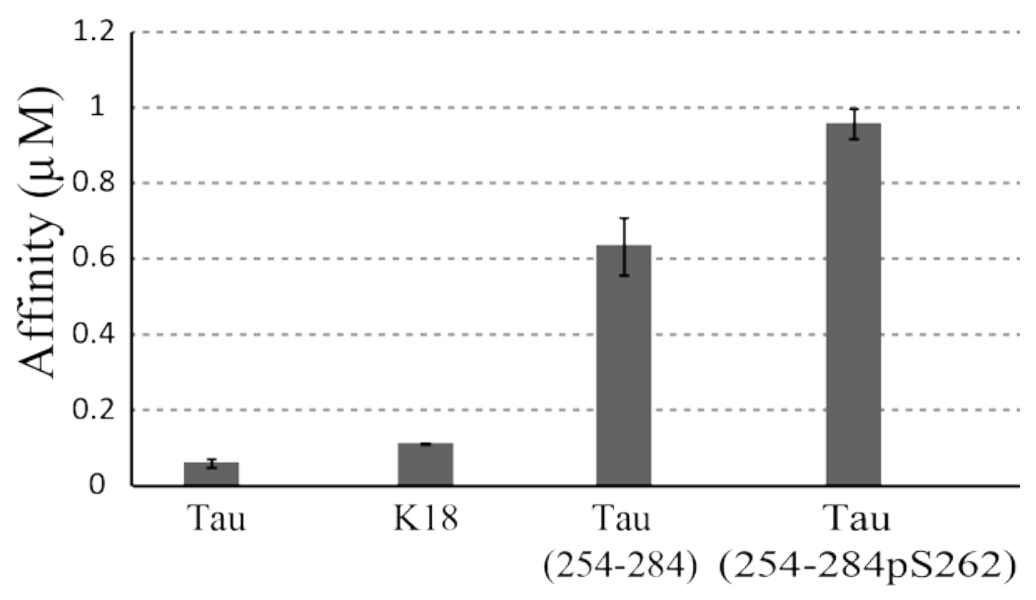

Figure 3.15. Affinities of Tau and different Tau constructs for binding to NBD-labeled F-actin.

The plot represents the calculated affinities based on the cahnge in intensity of NBD-labeled F-actin promoted by Tau or Tau constructs. The experiment was performed at low ionic stress and at $25{ }^{\circ} \mathrm{C}$. Error bars represent the standard deviation from three different experiments for every sample.

\subsubsection{Tau binds to the hydrophobic pocket of F-actin}

In order to gain insight into how Tau regulates the polymerization and structure of F-actin, we sought to obtain information about the Tau-binding site on F-actin. X-ray crystallography and electron microscopy showed that several actin-binding proteins bind to a hydrophobic pocket between subdomains 1 and 3 of G-actin (Carlier and Pantaloni 1997). One of these binding partners is cofilin, a $21 \mathrm{kDa}$ eukaryotic protein, which binds to F-actin with a $\mathrm{Kd}<0.05 \mu \mathrm{M}$ (Bobkov, Muhlrad et al. 2002) and results in disassembly of F-actin (Yonezawa, Nishida et al. 1987, Suarez, Roland et al. 2011). Cryo-electron microscopy further showed that the binding site of cofilin on F-actin is highly similar to its binding site on G-actin (Figure 3.16a) (Carlier and Pantaloni 1997). To test if the binding site of Tau on F-actin overlaps with that of cofilin, we added a 10-fold excess of cofilin to a solution containing ${ }^{15} \mathrm{~N}$-labeled Tau and 1.5-fold excess (with respect to Tau) of F-actin. For the resulting mixture, a ${ }^{1} \mathrm{H}-{ }^{15} \mathrm{~N}$ NMR correlation spectrum was recorded. After addition of cofilin, Tau's NMR resonance intensities and positions were similar to those in the absence of F-actin (Figure 3.16b), indicating that (i) Tau is no longer bound to F-actin and (ii) cofilin does not bind to Tau. 
a

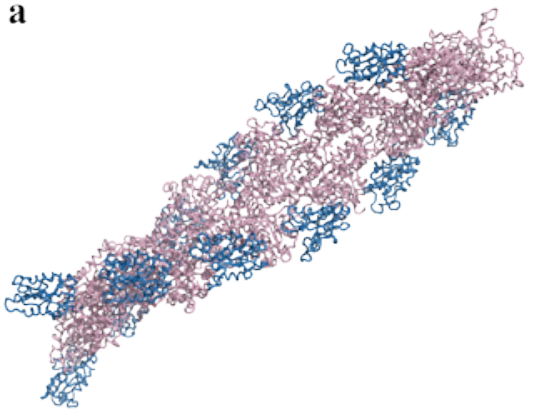

b

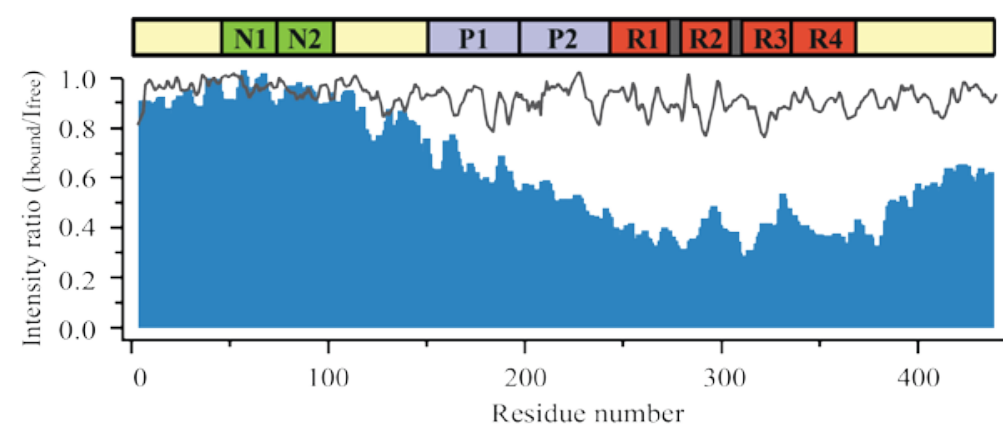

Figure 3.16. Tau competes with cofilin for binding to F-actin.

(a) Cofilin (blue) binds to and decorates actin filament (pink) severing F-actin at the junction between undecorated and cofilin-decorated regions of F-actin (PDB id 3J0S (Galkin, Orlova et al. 2011)). (b) Influence of a 10-fold excess of cofilin on ${ }^{1} \mathrm{H}^{-15} \mathrm{~N}$ cross-peak intensities of Tau in presence of F-actin (Tau/F-actin molar ratio of 1:1.5). Normalized signal intensities in the absence (blue) and presence of cofilin (grey) are shown.

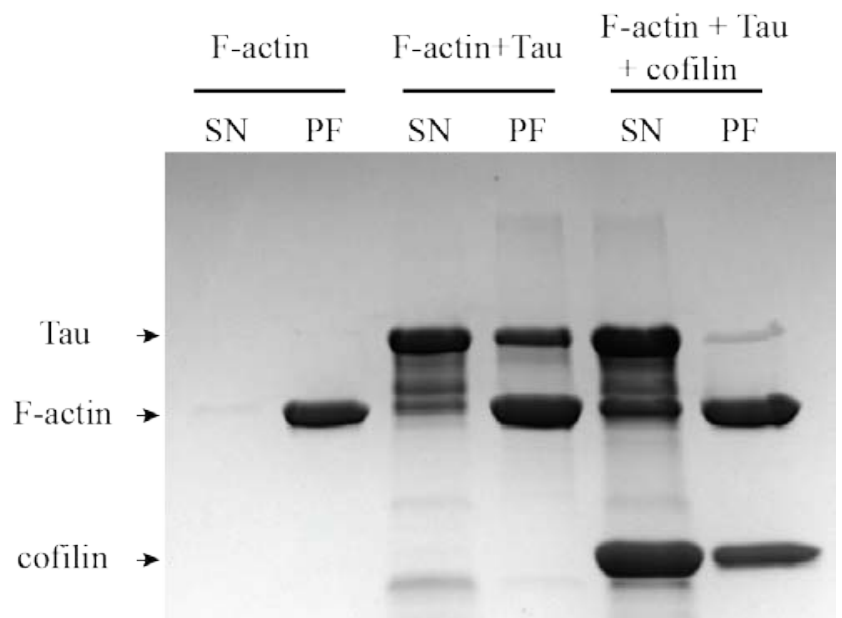

Figure 3.17. SDS gel-based competition effect between Tau and cofilin for binding to F-actin.

Centrifugation-based sedimentation of F-actin, F-actin+Tau (1.5:1) and F-actin+Tau+cofilin (molar ratio 1.5:1:10). Supernatants (SN) and pellets (PF) were loaded in different lanes of a 4-20 \% SDS gradient gel. Arrowheads from top to the bottom represent Tau, actin and cofilin bands, respectively.

To further support a competition between Tau and cofilin for binding to F-actin, we repeated the F-actin/Tau co-sedimentation assay in the presence of Cofilin (Figure 3.17). The ImageJ-based gel quantification showed that the $41 \%$ of Tau molecules appears to be bound to F-actin while the remaining $59 \%$ of Tau is still in the supernatant. On the other hand when cofilin is added to the solution only the $6.5 \%$ of Tau remains bound to actin because $35.5 \%$ of the total cofilin is 
bound to F-actin in a competitive manner. In agreement with the NMR data, addition of cofilin decreased the amount of Tau that co-precipitated with F-actin.

The cofilin-induced release of Tau from actin filaments might be caused by structural changes in actin upon binding of cofilin. Alternatively, cofilin and Tau share a common binding site, such that an excess of cofilin competes with the binding of Tau to actin. To distinguish between these two alternatives, we thus sought a way to attach MTSL to C374 in actin. C374 is in close proximity to actin's hydrophobic pocket, where cofilin binds (Figure 3.16a and Figure 3.18a). We thus incubated G-actin with MTSL, followed by polymerization of the MTSL-tagged protein into F-actin. Subsequently, ${ }^{15} \mathrm{~N}$-labelled Tau protein was added to reach a molar ratio of 1:2 (Factin in excess) and a ${ }^{1} \mathrm{H}^{15}{ }^{15} \mathrm{~N}$ HSC of Tau was recorded. Comparison with the Tau spectrum recorded in the presence of diamagnetic F-actin revealed a specific paramagnetic relaxation enhancement (PRE) profile (Figure 3.18b). The PRE profile contained seven peaks corresponding to residues L243-A246, K259-K267, V275-L284, S289-S293, S305-V313, S320H330 and K375-F378. Thus, these Tau residues come within $25 \AA$ of the paramagnetic center at C347 upon binding to F-actin.

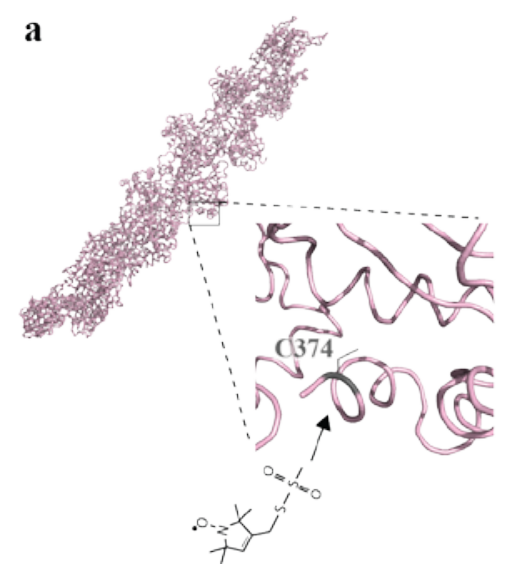

b

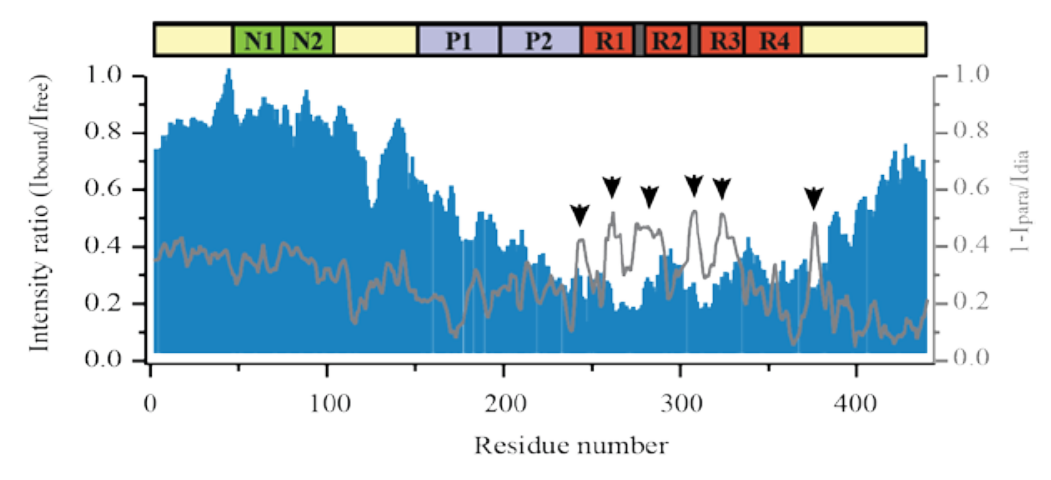

Figure 3.18. The PRE approach reveals that Tau binds nearby the hydrophobic pocket of F-actin.

(a) Every protomer on F-actin was covalently MTSL-labeled on C374. The figure is based on the cryoEM-based 3D structure of F-actin (PDB id 3J0S (Galkin, Orlova et al. 2011)) where cofilin was removed. (b) Sequence-specific paramagnetic broadening induced in Tau by C374-MTSL-tagged F-actin (grey line). $\mathrm{I}_{\text {para }}$ and $\mathrm{I}_{\text {dia }}$ are signal intensities observed for individual cross-peaks in two-dimensional ${ }^{1} \mathrm{H}$ ${ }^{15} \mathrm{~N}$ HSQCs of Tau in the presence of a two-fold excess of paramagnetic and diagmagnetic F-actin, respectively. For comparison, the attenuation of Tau signals by diamagnetic F-actin is shown (blue bars).

Observation of PRE broadening at the chemical shift of unbound Tau demonstrates that the Tau molecule exchanges between the F-actin-bound and free states. Because of the simultaneous 
observation of line broadening upon addition of diamagnetic F-actin (Figure 3.12 and Figure 3.18b), the exchange process is intermediate on the NMR chemical shift time scale. In addition to the specific PRE effects within and close to the repeat domain, a gradually increasing PRE effect was observed from approximately residue 170 towards the N-terminus. Because this region showed less sequence specific variation, we suggest that the broadening effect is the result of unspecific binding of the N-terminal half of Tau to the surface of F-actin. In addition, there might be contributions from paramagnetic broadening, when individual actin filaments come together in bundles. Notably, no PRE effect was observed at the C-terminus.

\subsubsection{Tau-F-actin interaction is mainly hydrophobic}

a

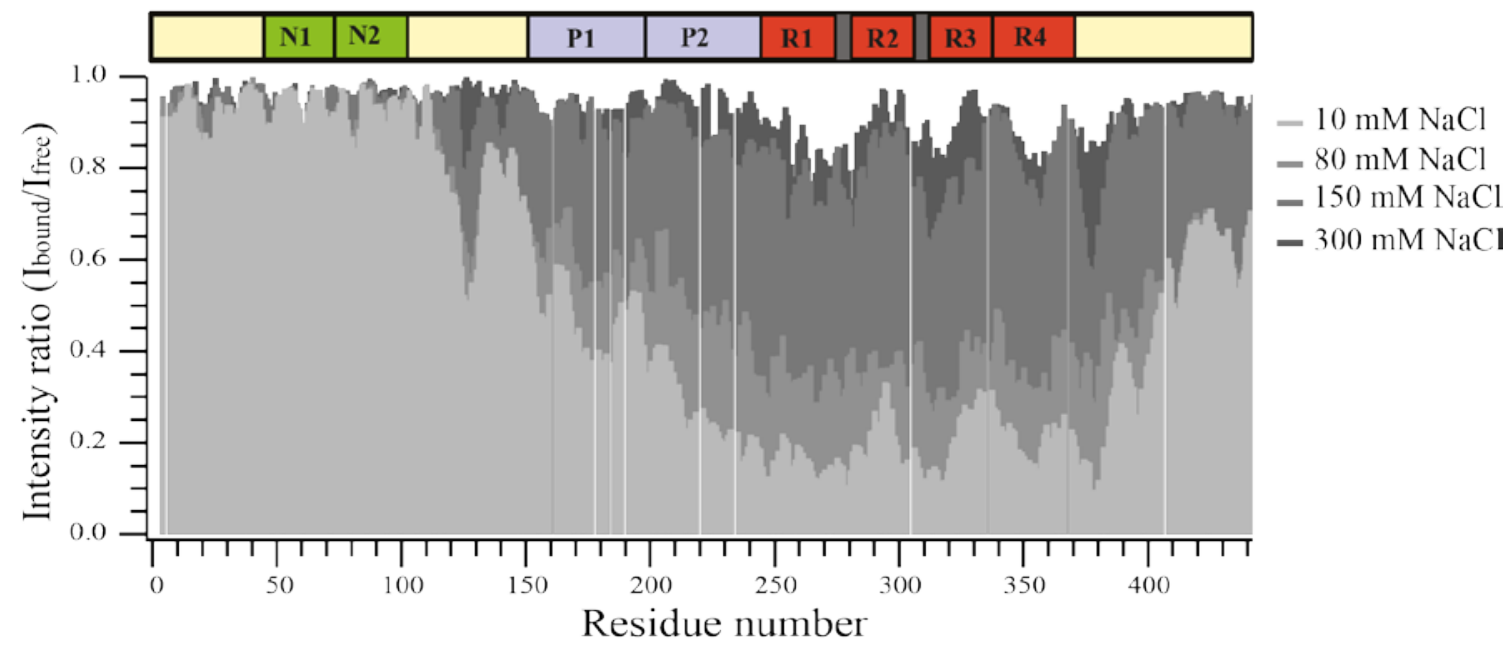

b

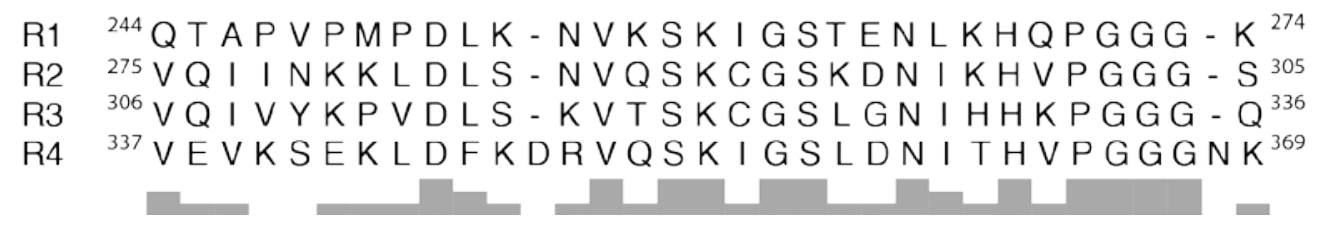

Figure 3.19. Salt titration experiment of Tau in presence of two-fold excess of F-actin.

(a), Superposition of ${ }^{1} \mathrm{H}^{15} \mathrm{~N}-\mathrm{HSQC}$ intensity ratios between Tau bound to F-actin $\left(\mathrm{I}_{\text {bound }}\right)$ and free in solution $\left(\mathrm{I}_{\text {free }}\right.$ ) as a function of the $\mathrm{NaCl}$ concentration. Organization of Tau domains is shown at the top. (b), sequence alignment of microtubule-binding repeats performed by Clustal Omega 1.2.3 (http://www.ebi.ac.uk/Tools/msa/clustalo)

To further analyze the nature of the binding between Tau and F-actin a titration experiment with different salt concentrations was performed. In this experiment the change of intensity a ${ }^{1} \mathrm{H}^{-15} \mathrm{~N}$ 
HSQC experiment from mixtures of Tau and two-fold excess of F-actin was analyzed as a function of $\mathrm{NaCl}$ concentration Figure 3.19a. The analysis revealed that the affinity of Tau decreases with the increase of the salt concentration because of the ionic stress. Nevertheless, hot spots from the MBD are still bound to F-actin at higher salt concentrations most probably due to the presence of hydrophobic interactions. If the sequences of different repeats are compared (Figure 3.19b) the high sequence similarity and the presence of several hydrophobic amio acids explain the hydrophobic nature of the interaction even at high ionic stress.

\subsubsection{Tau phosphorylation affects the interaction between Tau and F- actin}

Phosphorylation of Tau at S262 attenuates the Tau/F-actin interaction Tau protein is posttransnationally modified by phosphorylation, acetylation and several other modifications (Morishima and Ihara 1994, Wang, Grundke-Iqbal et al. 1996, Yuzwa, Shan et al. 2012, Tracy, Sohn et al. 2016). An important class of kinases that phosphorylate Tau at S262 and the other KXGS motifs in the repeat domain are the microtubule-associated protein/microtubule affinityregulating kinases (MARKs) (Drewes, Ebneth et al. 1997, Yoshida and Goedert 2012, Schwalbe, Biernat et al. 2013). We phosphorylated full-length ${ }^{15} \mathrm{~N}$-labeled Tau by MARK2, followed by NMR measurements in the absence and presence of F-actin. In contrast to the wild-type protein, little perturbation of the NMR signals in vicinity of the phosphorylation sites was detected (Figure 3.20). Residues such as S262 and S356 largely retained the NMR signal intensity in the presence of F-actin. However, residues in the proline-rich region, as well as in the segments V275-L284 and K294-V300, which do not contain MARK2-phosphorylation sites (Figure 3.20c), were broadened in presence of F-actin. In agreement with these results, attachment of a phosphate group to S262 in the peptide Tau(254-284) decreased the affinity of the peptide for Factin (Figure 3.15). 


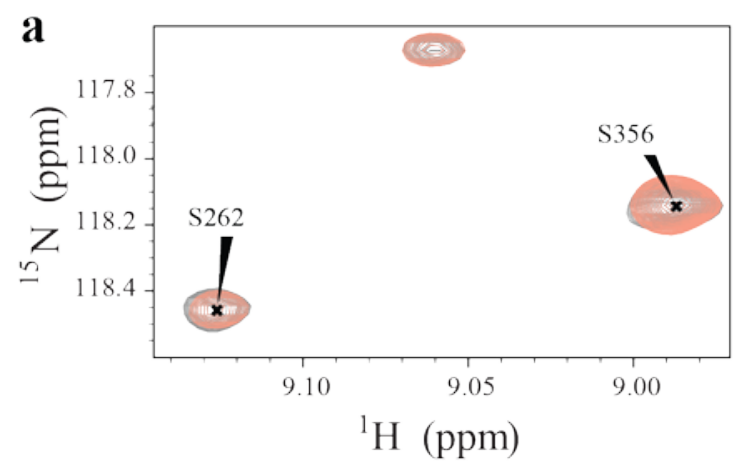

b

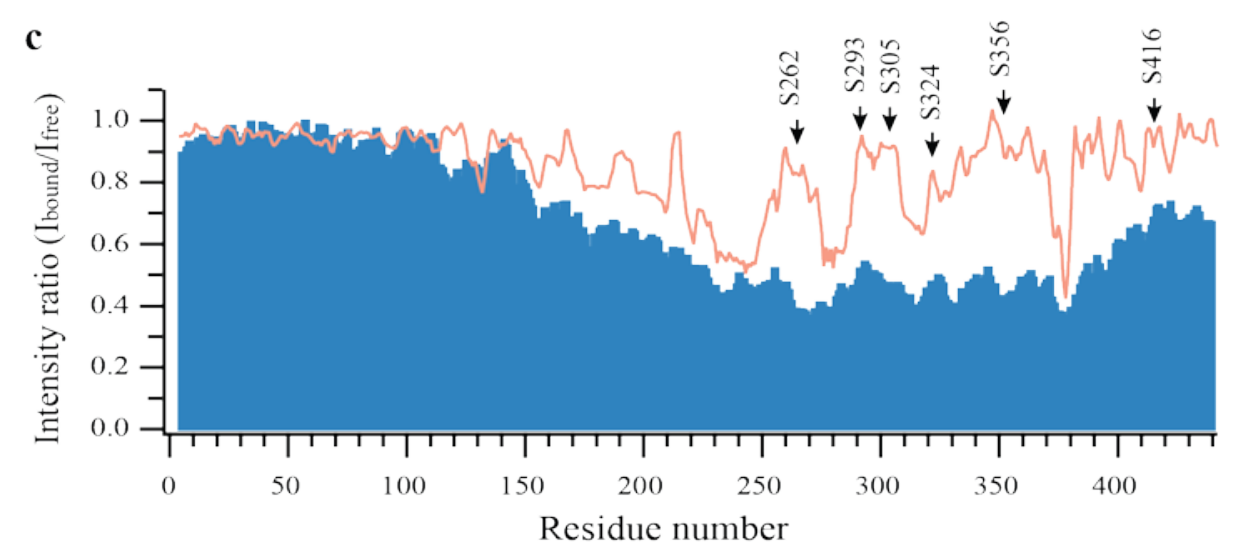

Figure 3.20. MARK2 phosphorylation decreases Tau's affinity for F-actin.

(a) Selected region from a ${ }^{1} \mathrm{H}-{ }^{15} \mathrm{~N}$ correlation spectrum of MARK2-phosphorylated Tau in the absence (gray) and presence of F-actin (orange; Tau/F-actin molar ratio of 1:1.5). (b) Selected region from a ${ }^{1} \mathrm{H}$ ${ }^{15} \mathrm{~N}$ correlation spectrum of wild-type Tau in the absence (gray) and presence of F-actin (blue; Tau/F-actin molar ratio of 1:1.5). (c) NMR signal broadening induced by F-actin in wild-type (blue bars) and MARK2-phosphorylated Tau (orange line). Tau residues phosphorylated by MARK2 are highlighted.

To further investigate the phosphorylation effect of Tau on F-actin, the extents of actin bundles promoted by Tau(254-284) and its phosphorylated version Tau(254-284pS262) were compared. In this experiment a 10-fold excess of peptide added to F-actin and loaded in a 4-20\% gradient gel after 30 minutes incubation (Figure 3.21). According to the gel quantification the $70 \%$ of Factin was bundled by $\mathrm{Tau}(254-284)$ while the other $25 \%$ remains as single filaments. If Tau(254284pS262) is used only 7\% of actin is in bundles while 75\% of actin remains as single filament. In agreement with Figure 3.15 the result clearly suggests that the phosphorylation of Tau(254284) on S262 decreases the bundling effect. This further support the effect in the intensity plot on Figure 3.20d were S262 appears to be detached from F-actin surface. 


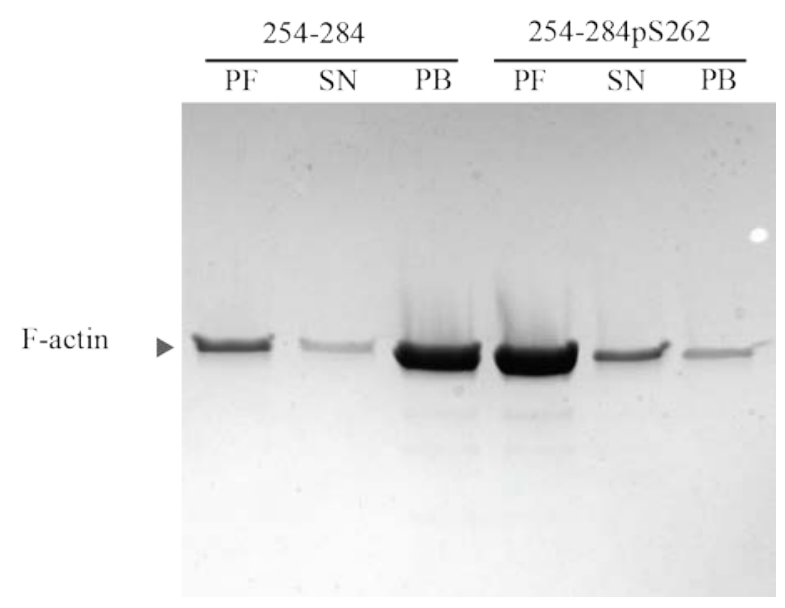

Figure 3.21. SDS-PAGE of phosphorylated and unphosphorylated Tau(254-284) in presence of Factin.

Phosphorylation of S262 decreases the ability of Tau(254-284) to bundle F-actin. The arrowhead indicates the location of actin on the SDS gel.

\subsection{NMR-based structure calculation of Tau in complex with F- actin}

So far we have shown that Tau binds to F-actin with a high affinity by means of the MBD and P2. We have also seen, by means of competition experiments that it binds on the hydrophobic pocket of actin, which is exposed to the solvent in the filament conformation. This interaction is very important for the Tau-promoted bundles stability that has been previously described (Fulga, Elson-Schwab et al. 2007). Provided that Tau is disordered in solution it is important to understand the mechanism involved in F-actin stabilization. Intrinsically disordered proteins often contain multiple binding sites with each binding site only providing low affinity for a target protein. In case of F-actin, the high molecular weight of actin filaments further complicates structural analysis of the complex between actin filaments and MAPs.

Using a combination of NMR spectroscopy and other biophysical methods, we here dissect the regions in both Tau and F-actin that are important for complex formation. We then provide highresolution information about the structural changes that occur when Tau binds to F-actin and show that conformational changes in specific regions, which are separated by flexible linkers, are responsible for bundling of actin filaments as well as for cross-linking of actin filaments with 
microtubules. Our results thus clarify the nature of interaction between actin and MAPs and the actin/microtubule crosstalk.

\subsection{1. $\quad$ Selection of Tau peptides}

While analyzing the intensity plots for Tau and F-actin interaction it is clear that linear motives of Tau are more affected than others. These linear motives are also connected by flexible linkers where the binding seems to be weaker. This allowed us to use the so-called "divided and conquer strategy” which consist in taking shorter fragments of Tau to derive the structure of full-length Tau in bound state to F-actin. Based on this idea the peptides Tau(254-290), Tau(292-319) and an even shorter peptide Tau(254-268) were taken to determine their 3D structures.

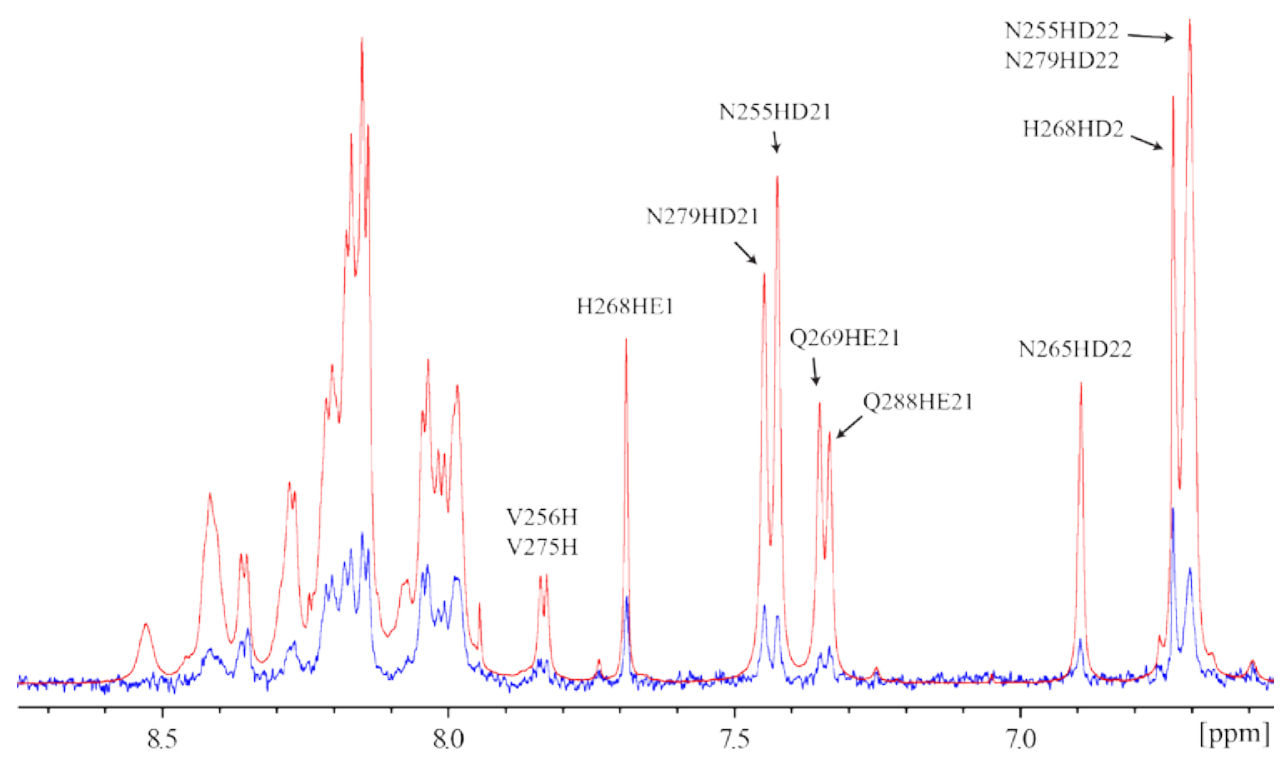

Figure 3.22. Tau(254-290) bind to $\mathrm{F}$-actin in a fast exchange regime.

Selected region of 1D STD NMR spectrum of Tau(254-290) in presence of F-actin with a selective saturation of F-actin at $1 \mathrm{ppm}$ (blue). The 1D spectrum from the sample is also show (red). Some of the affected protons from the peptide upon selective saturation are labeled.

To determine whether these peptides are candidates for NMR-based structure determination, STD NMR experiments were performed. The efficacy of the STD method depend on the weak strength of the binding between molecules resulting in fast exchange regime between bound and free states of the ligands (Mayer and Meyer 1999). The STD NMR spectrum of Tau(54-290) bound to F-actin is shown in Figure 3.22. The appearance of STD peaks reveals that the peptide is bound to F-actin and the exchange is fast enough to detect transferred NOE peaks. Similarly other peptides used for structure calculation where also tested. 


\subsubsection{Peptides assignment}

The primary requirement of the NMR-based structure determination is the complete resonance assignment of the spectra. The resonance assignment of the spectra was done using the standard Wüthrich method, which was explained on section 2.10.4, for all the peptides analyzed in this work. The sequential connectivity form $i^{\text {th }}$ residue to $\mathrm{i}+1^{\text {th }}$ residue was in the TOCSY spectra were performed with a help of NOESY spectra as can be seen on Figure 3.23 for Tau(254-290) (panel a) and Tau(292-319) (panel b). In the figure only few resonances and their respective connections are shown for clarity. Thus the complete resonance assignments of individual amino acids of Tau(254-268), Tau(254-290) and Tau(292-319) were performed unambiguously.
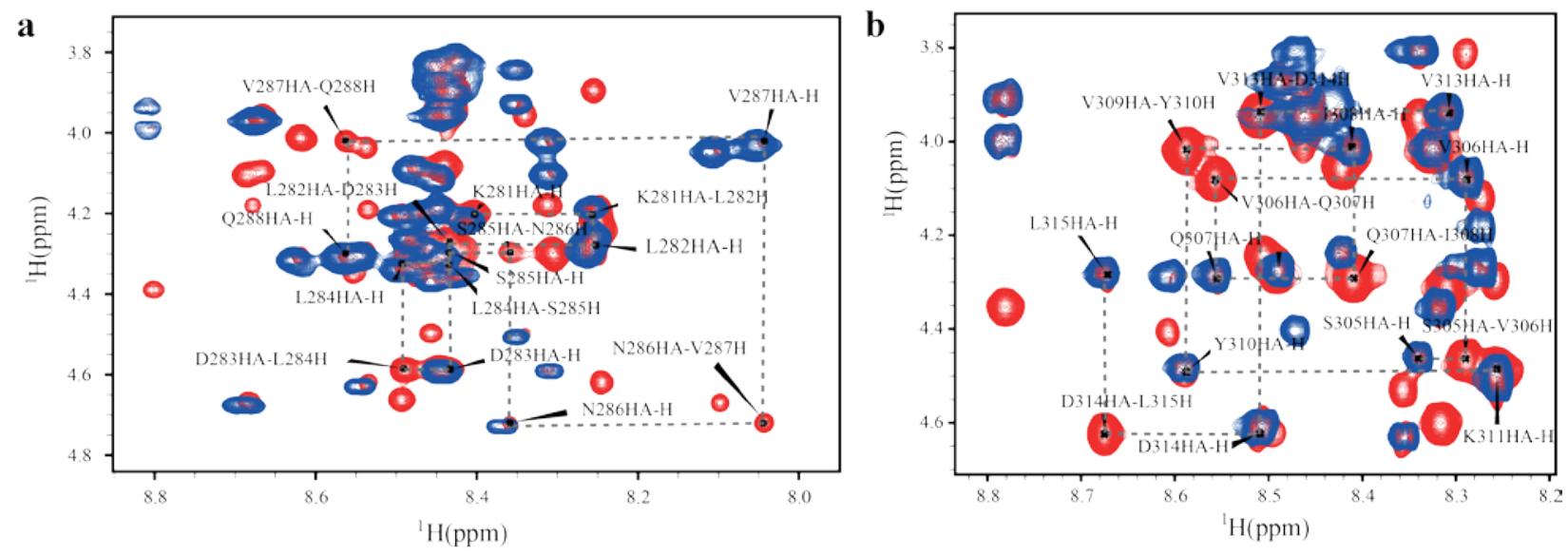

Figure 3.23. Resonance assignment of Tau peptides.

The resonance assignment of the peptides Tau(254-290) and Tau(292-19) are presented in (a) and (b) respectively. In both cases the assignment was performed by following the sequential connection (dashed lines) between residues is a TOCSY spectra (blue) with the help of a NOESY spectrum (red). Only some residues and their respective connections are shown for clarity.

\subsubsection{Structure calculation of Tau(254-268) bound to F-actin}

In order to derive the 3D structures of Tau(254-268) in bound state to F-actin, we used the socalled transferred NOE approach which have been widely used to determine the structure of small ligands in complexes with high molecular weight binding partners (Andrianantoandro and Pollard 2006, Kadavath, Jaremko et al. 2015). To do so, two-dimensional Nuclear Overhauser Effect (NOE) spectra of Tau peptide in the absence and presence of F-actin were acquired to a sample containing a 30:1 molar ratio (peptide in excess) with 100 ms mixing time. Two different 
regions of the superposition of the two spectra in absence (blue) and presence (red) of F-actin are showed in Figure 3.24. In both cases NOE peaks appeared because of the transferred magnetization from peptide in bound to free state upon addition of F-actin. These additional peaks in the complex are a consequence of the medium-range interactions $(1<|\mathrm{i}-\mathrm{j}| \leq 4)$ as a result of the conformational change of the peptide bound to F-actin. When the peptide is free in solution its structure is disordered and only sequential and intra residue NOES are visible.
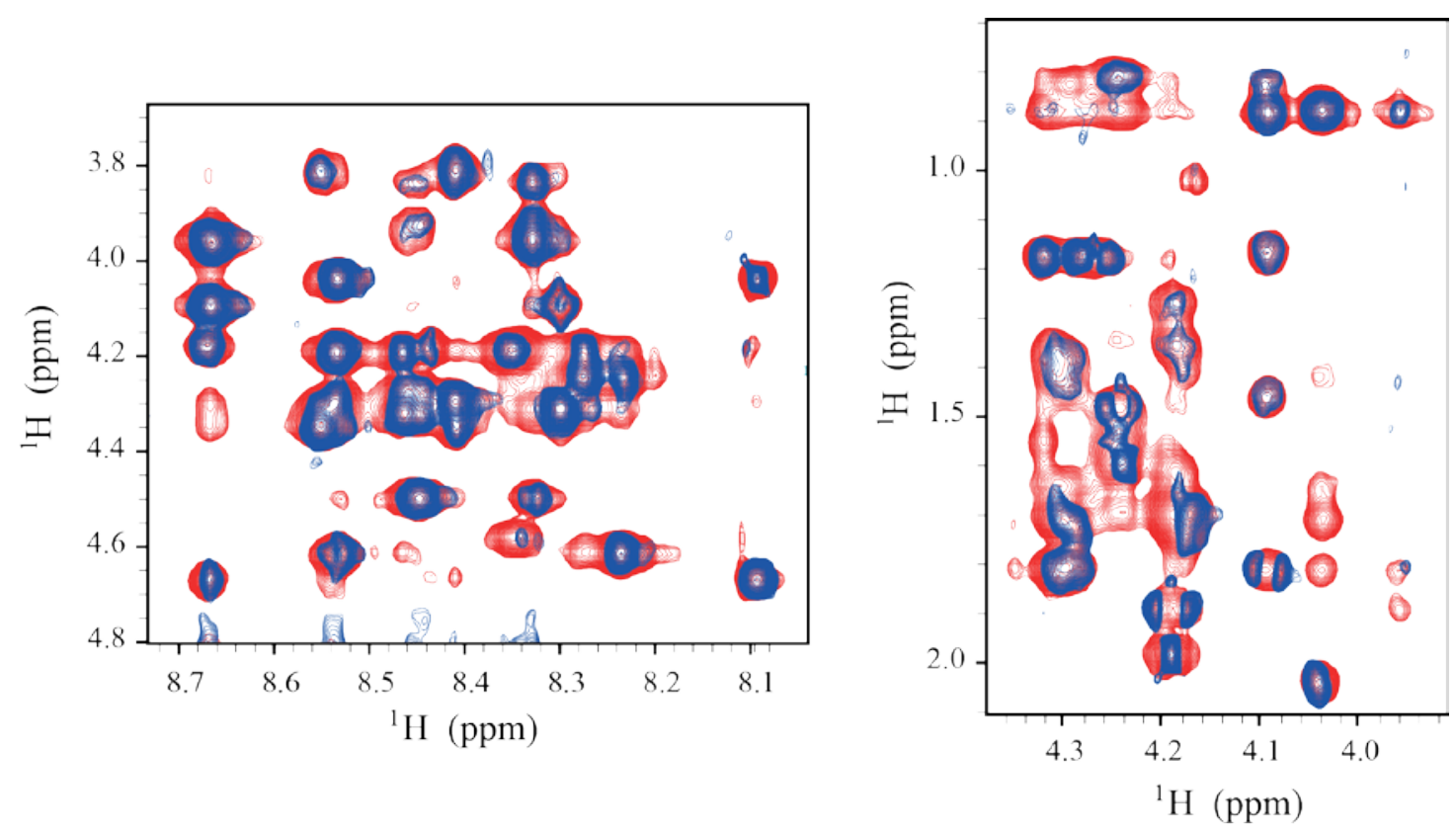

Figure 3.24. Superimposed NOE spectra from Tau(254-268) in absence (blue) and in presence (red) of F-actin.

New NOE peaks appear when F-actin is present because of the conformational change of the peptide upon binding.

The analysis of the tr-NOEs indicates the presence of a number of medium-range interactions between almost all the residues when the peptide is bound to F-actin. However, only intra and sequential NOEs are observed when the peptide is in solution. The appearance or new cross peaks reveals the presence of a structured conformation of the peptide induced by the binding. Upon analyzing the full spectrum 313 distance restraints were collected as can be seen in Table 3.1. Among these distances restraints 132 were intra residue, 143 sequential and 38 medium range interactions. 
Table 3.1. Structural statistics of Tau(254-268) bound to F-actin

\begin{tabular}{ll} 
Structural statistics for 20 final conformers of Tau (254-268) bound to F-actin \\
\hline Number of restraints & 313 \\
Intra residue NOE $(|\mathrm{i}-\mathrm{j}|=0)$ & 132 \\
Sequential NOE $(|\mathrm{i}-\mathrm{j}|=1)$ & 143 \\
Medium range NOE $(<1|\mathrm{i}-\mathrm{j}| \leq 4)$ & 38 \\
NOE violations $>0.5 \AA$ /structure & 0 \\
Ramachandran plot statistics & \\
Residues in most favored regions & $82.1 \%$ \\
Residues in additionally allowed regions & $10.4 \%$ \\
Residues in generously allowed regions & $5.4 \%$ \\
Residues in disallowed regions & $2.1 \%$ \\
RMSD from the average structure & \\
Backbone atoms & \\
Full peptide & $0.99 \AA$ \\
Heavy atoms & $1.62 \AA$
\end{tabular}

The initial structure calculations of Tau(254-268) were performed with Cyana 3.97 using distances restraints derived from Tr-NOESY experiment. Subsequently the structure was refined using XPLOR-NIH using the standard simulated annealing protocol. In order to avoid potential miscalibration of NOE intensities, only medium range NOE contacts were used. Lower and upper limits were set to $1.8 \AA$ and $6.0 \AA$ respectively. 400 structures were calculated and the 20 lowest-energy conformers were selected for analysis using the protein structure validation software suite (PSVS) (Bhattacharya, Tejero et al. 2007). No violations above $0.5 \AA$ were observed in these structures. Statistic from Ramachandran plot as well as the RMSD from the average structure reveals that the structure was properly calculated (see Table 3.1). 
A

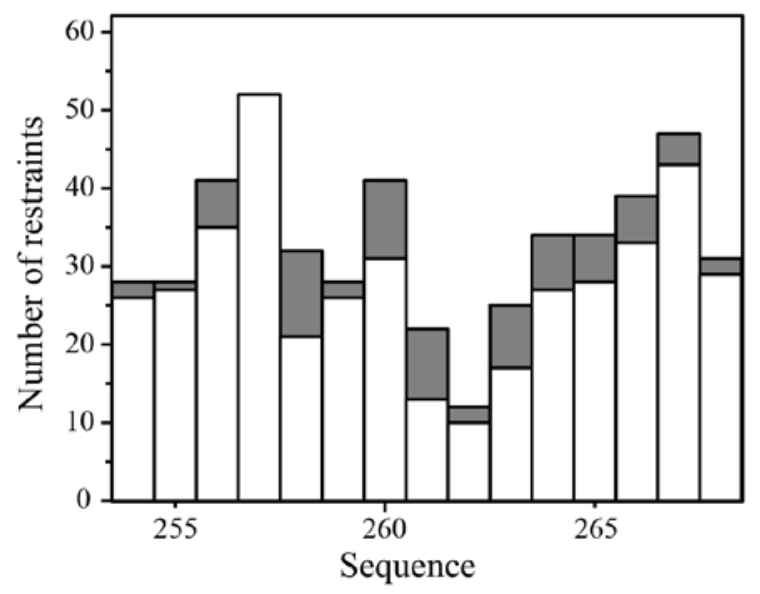

B
${ }^{254}$ KN V K S K I G S T EN L K H

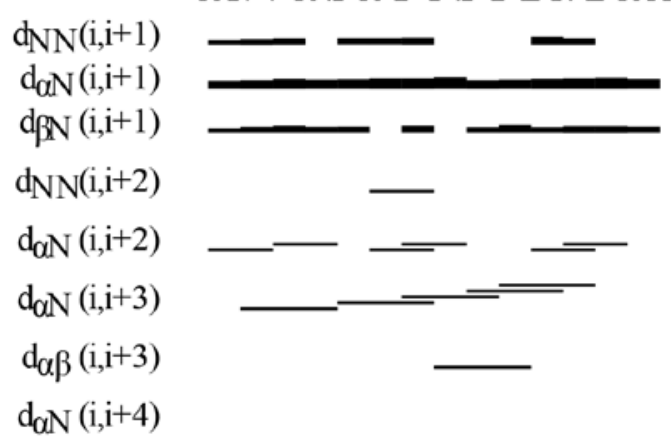

Figure 3.25. Distribution of the NOE interactions of Tau(254-268) bound to F-actin.

(a) Distribution of the distance constraints as a function of the residue number of the peptide. Upper distance limits are classified according to their range as intra and sequential constraints (white bars) and medium-range (grey bars). (b) Overview of sequential and medium-range NOEs in various secondary structural elements. Horizontal lines connecting the corresponding proton positions indicate short distances between atoms $(\leq 6 \AA)$. The thickness of the lines is proportional to the intensity of the observed NOE.

The NOE connectivity, which is the hallmark for the secondary structure element and the number of NOEs per each residue are shown in Figure 3.25a. Here white bars represent sequential NOEs while medium-range interactions are represented by grey bars.

a

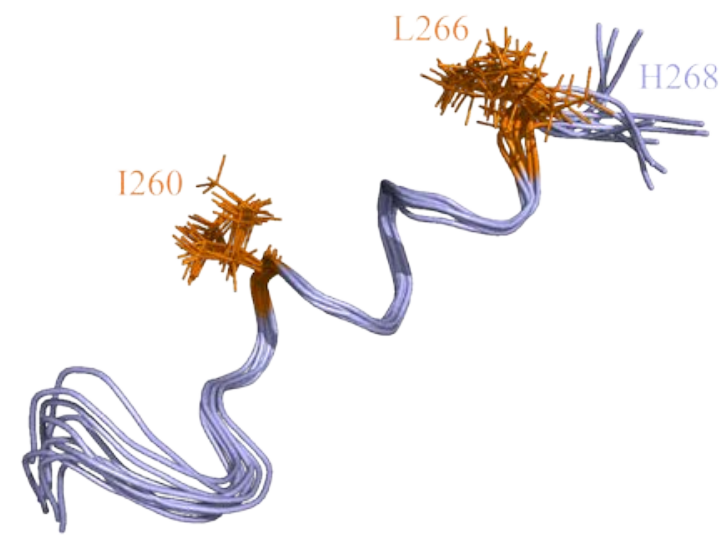

b

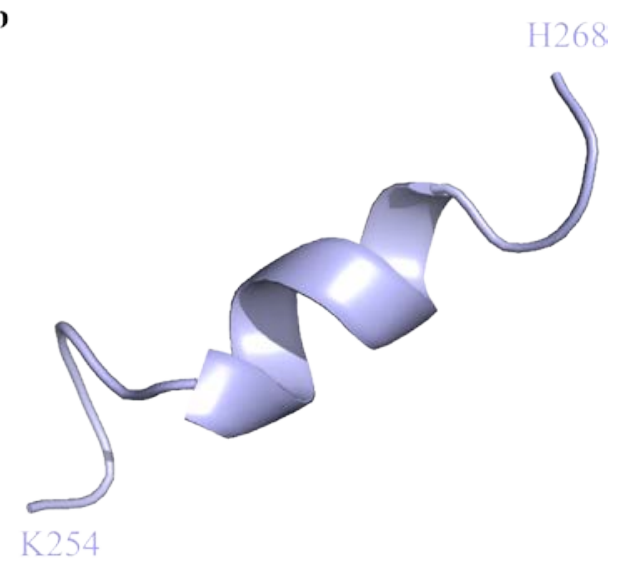

Figure 3.26. Structure of Tau(254-268) bound to F-actin.

(a) Backbone superposition of the best 10 structures of the peptide bound to F-actin as calculated by trNOESY experiments. Hydrophobic residues are highlighted in yellow. (b) Cartoon representation of the $\alpha$-helical structure adopted by the peptide upon binding. 
Note, especially in Figure 3.25b, that secondary structure elements are distributed around the whole sequence meaning that the peptide is fully structured. A ribbon representation of the best 10 calculated conformers are shown in Figure 3.26a. Interestingly hydrophobic residues I206 and L266 are pointing towards the same direction, which could be an important clue about the nature of the binding. The structure validation analysis revealed that the peptide adopts an $\alpha$-helix conformation between residues I260 and E264 as it can be seen in Figure 3.26b.

\subsubsection{Structure calculation of Tau(254-290) bound to F-actin}

Provided that Tau(254-268) does not promote the bundle formation of F-actin (Figure 3.14d) we decided to determine the structure of a larger construct having the bundling capability. With this information we might be able to derive a connection between structure and function. Therefore we decided to calculate the structure of the peptide Tau(254-290), which is 22 residues larger than Tau(254-268) promotes F-actin bundle (Figure 3.14a).

The sequential assignment of this peptide was done as in the previous case by using a combination of a TOCSY and a NOESY spectrum. For the experiment a sample containing 800 $\mu \mathrm{M}$ peptide and $26.6 \mu \mathrm{M}$ F-actin was prepared in a 30:1 ratio. To discard any diffusion effect due to the high molecular weight of the complex 5 different NOE spectra were acquired at different mixing times ranging from 50 up to $250 \mathrm{~ms}$. Analysis of NOE signal intensities from cross-peaks S262HA-N265H and D283HA-N286H as a function of NOE mixing time showed that spin diffusion, does not significantly contribute to NOE intensities at used mixing times (Figure 3.27a). For the structure calculation the spectrum acquired with a mixing time of $100 \mathrm{~ms}$ was used. As in the previous case, only medium range NOE contacts were used with the lower and upper limits set to $1.8 \AA$ and $6.0 \AA$ respectively. Two different regions of the spectra from Tau(254-290) are shown in Figure 3.27b and Figure 3.27c. In both cases NOE peaks appeared because of the transferred magnetization from the peptide in bound to free state upon addition of F-actin. 
a

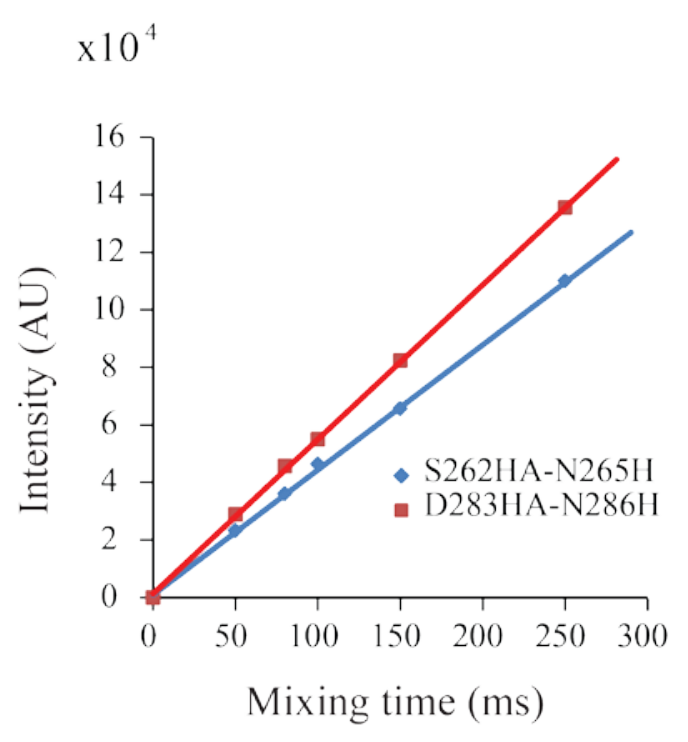

b
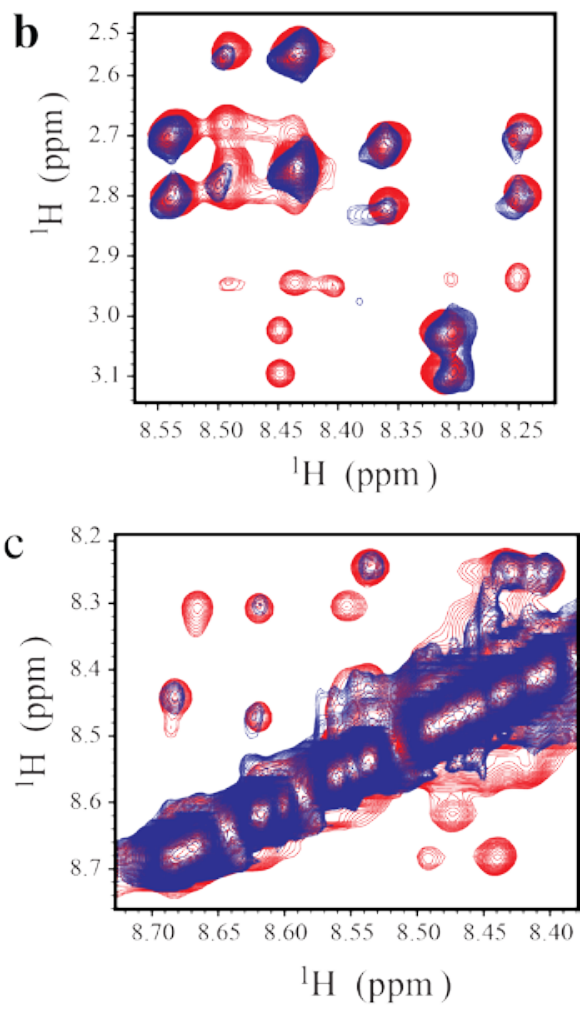

Figure 3.27. NOE intensity buildup curve for two NOE cross peaks.

(a) The buildup curves for the NOE cross-peaks S262HA-N265H (blue) and D283HA-N286H (red) are presented as a function of the mixing times 50, 80, 100, 150 and $250 \mathrm{~ms}$. (b,c), Two regions of superimposed NOESY spectra from Tau(254-290) in absence (blue) and in presence (red) of F-actin.

A total of 778 distance restraints were collected with 313 intra-residue, 283 sequential and 182 medium range interactions as can be seen in Table 3.2.

The distribution of the restraints are presented in Figure 3.28a where color code is similar to the previous case. In the histogram white bars represent sequential NOEs while medium-range interactions are represented by grey bars. C- and N-termini are structured whereas a flexible region is located at the middle of the sequence (Figure 3.28b). This flexible region correlates with the PGGG motive from P270 to G273.

Structure validation revealed a nice Ramachandran statistic (see Table 3.2) with $91.2 \%$ of the residues in the most favored region whereas only $1.4 \%$ of them were in disallowed regions. The $\mathrm{N}$-terminal part of the 20 calculated conformers were properly aligned with a RMSD of $1.139 \AA$ if only backbone atoms are considered or $1.966 \AA$ for heavy atoms. The C-terminal part is also structured although the RMSD for aligned residues from I278 to V287 is a bit higher. This may 
indicate that the C-terminal part is transiently structured when bound to F-actin or the binding is less strong in comparison to the N-terminus.

Table 3.2. Structural statistics of Tau(254-290) bound to F-actin

\begin{tabular}{|c|c|}
\hline Number of restraints & 778 \\
\hline Intra residue NOE $(|\mathrm{i}-\mathrm{j}|=0)$ & 313 \\
\hline Sequential NOE $(|i-j|=1)$ & 283 \\
\hline Medium range NOE $(1<|\mathrm{i}-\mathrm{j}| \leq 4)$ & 182 \\
\hline NOE violations $>0.5 \AA /$ structure & 0 \\
\hline \multicolumn{2}{|l|}{ Ramachandran plot statistics } \\
\hline Residues in most favored regions & $91.2 \%$ \\
\hline Residues in additionally allowed regions & $6.4 \%$ \\
\hline Residues in generously allowed regions & $0.0 \%$ \\
\hline Residues in disallowed regions & $1.4 \%$ \\
\hline
\end{tabular}

\begin{tabular}{cll}
\hline RMSD from the average structure & Residues (256-267) & Residues (278-287) \\
\hline All backbone atoms $(\AA)$ & 1.139 & 1.864 \\
Heavy atoms $(\AA)$ & 1.966 & 2.113
\end{tabular}

a

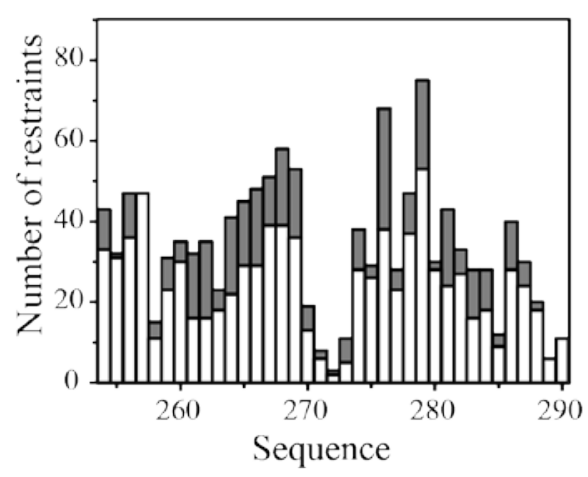

b

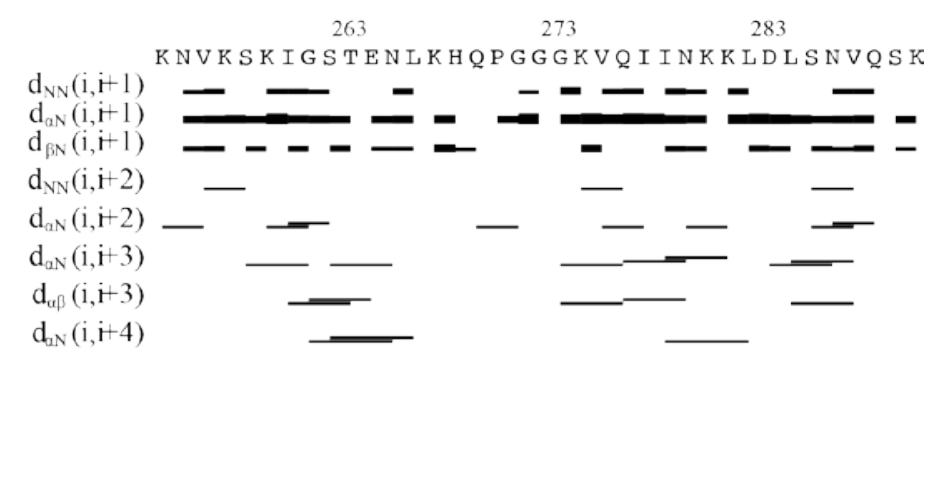

Figure 3.28. Distribution of the NOE interactions for Tau(254-290).

(a) Distance constraints distribution as a function of the residue number of the peptide. Upper distance limits are classified according to their range as intra and sequential constraints (white bars) and medium range (grey bars). (b) Overview of sequential and medium-range NOEs in various secondary structural elements. 
a

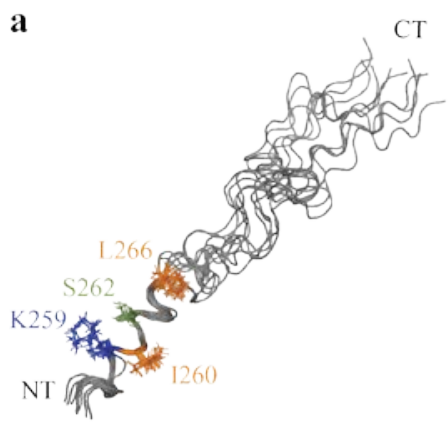

b

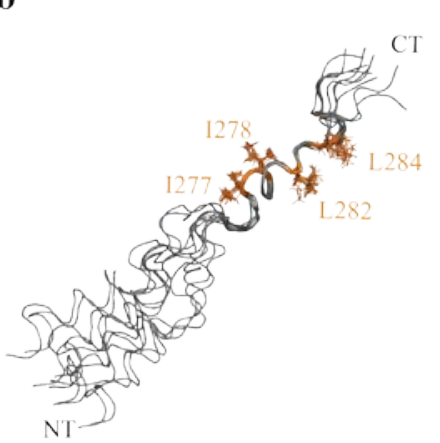

c

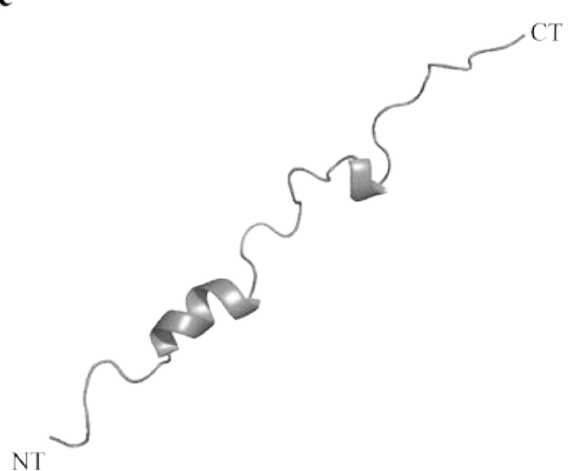

Figure 3.29. Ribbon representation of the best 10 conformers from Tau(254-290).

(a) 10 lowest-energy conformers of F-actin bound Tau(254-290). Structures were aligned from N255 to H268. Hydrophobic residues are colored orange. In addition, K259 (blue) and S262 (light green) are highlighted. (b) 10 lowest-energy conformers of F-actin bound Tau(254-290) aligned from residue Q276 to V287. (c) Cartoon representation of Tau(254-290) in bound state to F-actin.

The best 10 calculated conformers are shown in Figure 3.29a and Figure 3.29b. The peptide binds to F-actin in an extended conformation, which is in agreement with the lack of long-range interaction. Protein structure validation revealed a short $\alpha$-helix in the $\mathrm{N}$-terminus from residue G261 to K267 Figure 3.29c. If the 10 conformers are aligned, hydrophobic residues I260 and L266 are oriented in the same direction while S262 remains in the same plane. In this specific orientation K259 is pointing toward the opposite direction. Downstream of K267 an unstructured region follows, which separates the K259-K267 helix from a second helical region formed by V275 to L284 (Figure 3.29b). This region contains the aggregation-prone hexapeptide ${ }^{275}$ VQIINK ${ }^{280}$. Besides the hydrophobic hexapeptide, two additional hydrophobic residues, L282 and L284 are present, resulting in a larger hydrophobic surface of the helix (Figure 3.29b). Due to the lack of long-range contacts, the relative orientation of the two $\alpha$ helices was not defined in the 3D structure. Instead, the presence of a proline residue and three glycines in the linker region (the conserved PGGG motif) suggests that the intervening region remains flexible, enabling a variety of different relative orientations of the two helices.

\subsubsection{Structure calculation of Tau(292-319) bound to F-actin}

The structure calculation for a consecutive peptide Tau(292-319) was also performed. The sequential assignment of this peptide was performed using the same methodology as before. For the structure calculation of the Tau peptide a mixture of Tau(292-319) and F-actin (30:1 ratio) 
was prepared. Upon analyzing the NOE spectrum a total of 385 restraints were obtained. From them 150 were intra-residue, 166 sequential and 69 medium-range contacts as shown in Table 3.3.

Structure validation revealed that $71.9 \%$ of the residues were in the most favored regions whereas $5.7 \%$ of them were in disallowed regions (Table 3.3). The N-terminal part of the 20 calculated conformers between residue S293 and V300 can be aligned with a RMSD of $0.53 \AA$ considering only the backbone atoms or $1.35 \AA$ for heavy atoms. The C-terminal part is also structured with RMSDs of $0.83 \AA$ and $1.49 \AA$ for backbone atoms and heavy atoms respectively. The NOE connectivities and the number of NOEs per residue are shown as a histogram in Figure 3.30a. Unlike the previous peptides a major number of distance restraints are observed on the Cterminus than for the N-terminus. The restraints distribution through the sequence (Figure 3.30b) also shows that the C-terminus is more structured in agreement to what is shown in Figure 3.30a.

Table 3.3. Structural statistics of Tau(292-319) bound to F-actin

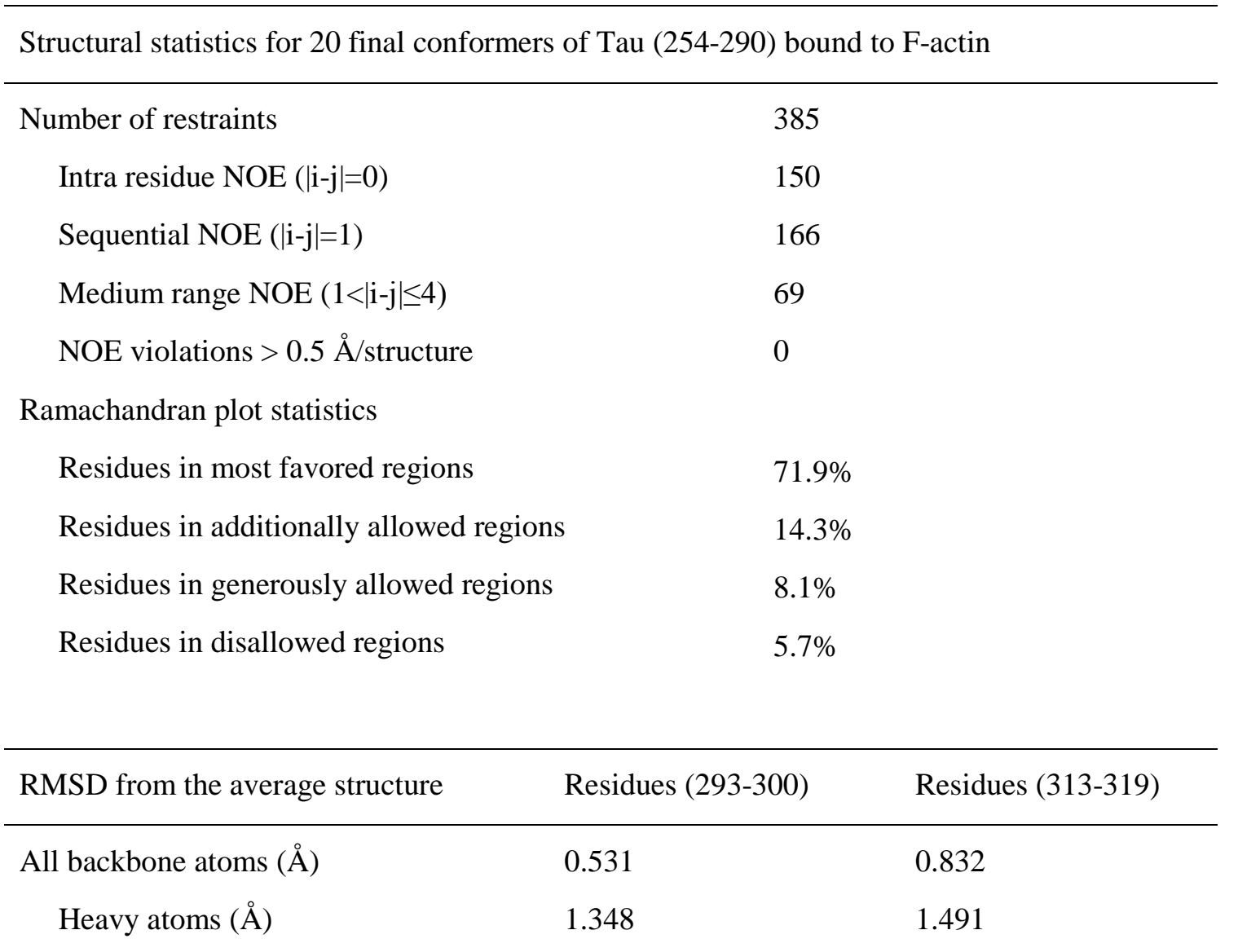


a

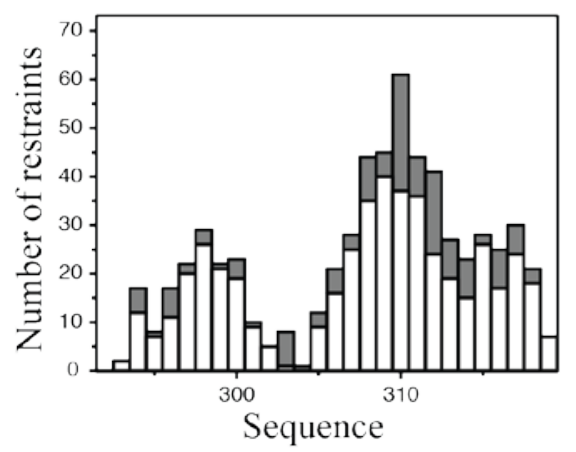

b

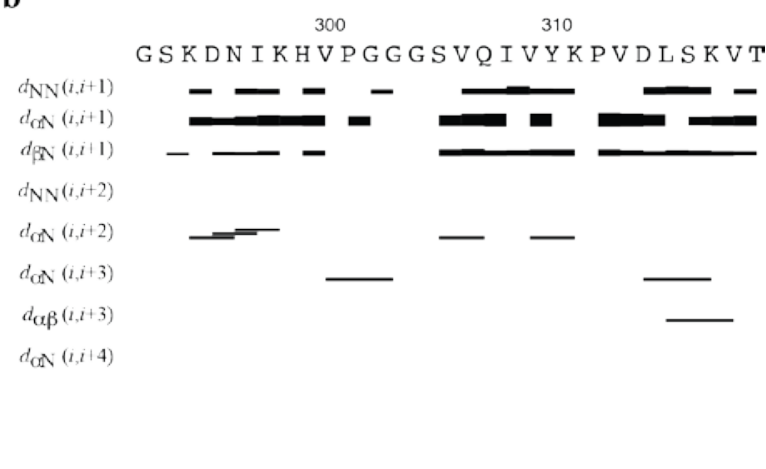

Figure 3.30. Distribution of the NOE interactions for Tau(292-319).

(a) Distribution of the distance constraints as a function of the residue number of the peptide. Upper distance limits are classified according to their range as intra and sequential constraints (white bars) and medium-range (grey bars). (b) Overview of sequential and medium ranges NOEs in various secondary structural elements. Horizontal lines connect the protons, which are separated by short distances $(\leq 6 \AA)$ while the thickness of the lines is proportional to the intensity of the observed NOE.

The best 10 calculated conformers are presented in Figure 3.31a and b. The peptide binds to Factin in an extended conformation due to no long-range interactions were observed. Protein structure validation of 20 different conformers reveals a short $\alpha$-helix in the C-terminus from residue L315 to V318. A cartoon representation of the peptide is shown in Figure 3.31c where hydrophobic residues are highlighted at the C-terminal part.

a

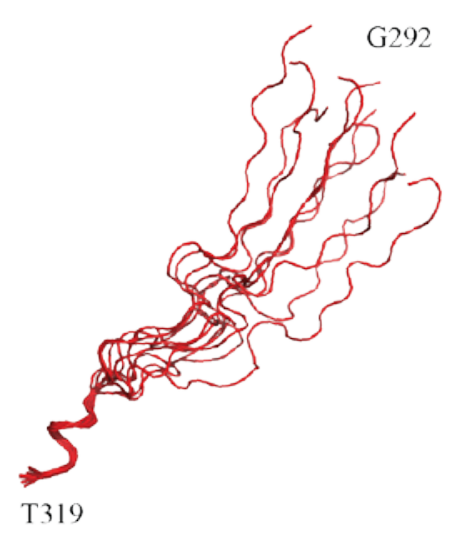

b

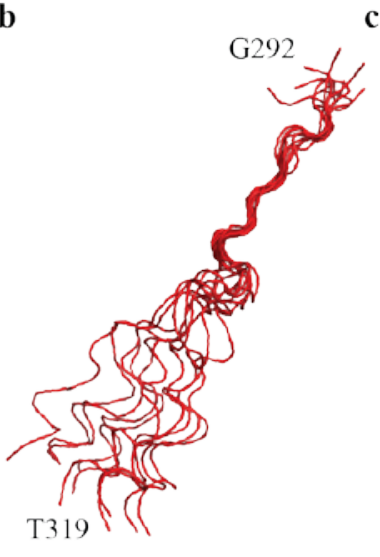
c

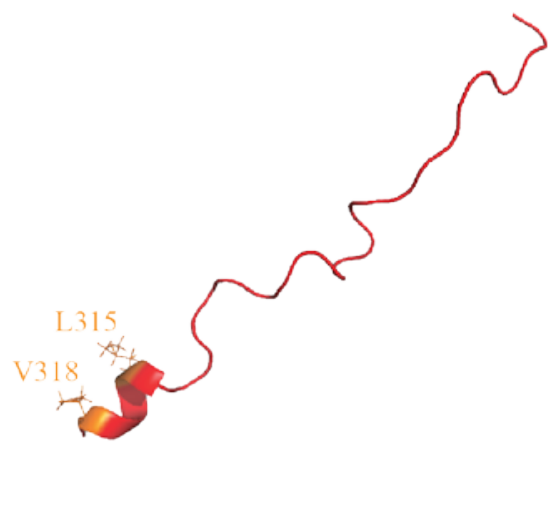

Figure 3.31. Ribbon representation of the best 10 conformers from Tau(292-319).

(a) The conformers were aligned toward the C-terminus from V313 to T319 where a short $\alpha$-helix from residues L315 to V318 can be seen. (b) Similarly, the N-terminal part was aligned from residue S293 to V300. (c) Cartoon representation of Tau(292-319) in bound state to F-Actin. Hydrophobic residues on the $\alpha$-helix are highlighted. 


\subsection{Docking the Tau(254-290) structure on F-actin surface}

To derive a structural model for the Tau/F-actin binding site, we integrated the information that was obtained from the peptide Tau(254-290) with the 3D structure of cofilin-decorated F-actin (Figure 1.10a). To this end, the NOE-based 3D structure of Tau(254-290) was docked to the solvent-exposed, hydrophobic pocket of actin within the actin filament structure using the software Haddock (Gorbatyuk, Nosworthy et al. 2006). The selected active residues on F-actin were listed in Materials and Methods and are highlighted in cyan color on Figure 3.32. Surrounding residues were considered as passives while the full-length peptide was docked. Haddock clustered 192 complex structures in four clusters, representing $96.0 \%$ of the waterrefined Haddock models. Within the highest scoring cluster (Table 3.4), the N-terminal $\alpha$-helix of Tau(254-290) was bound to the hydrophobic pocket of F-actin (Figure 3.33a). In agreement with the importance of hydrophobic interactions (Figure 3.19), Tau residues I260 and L266 made hydrophobic contacts with F352 and I345 from actin, respectively.

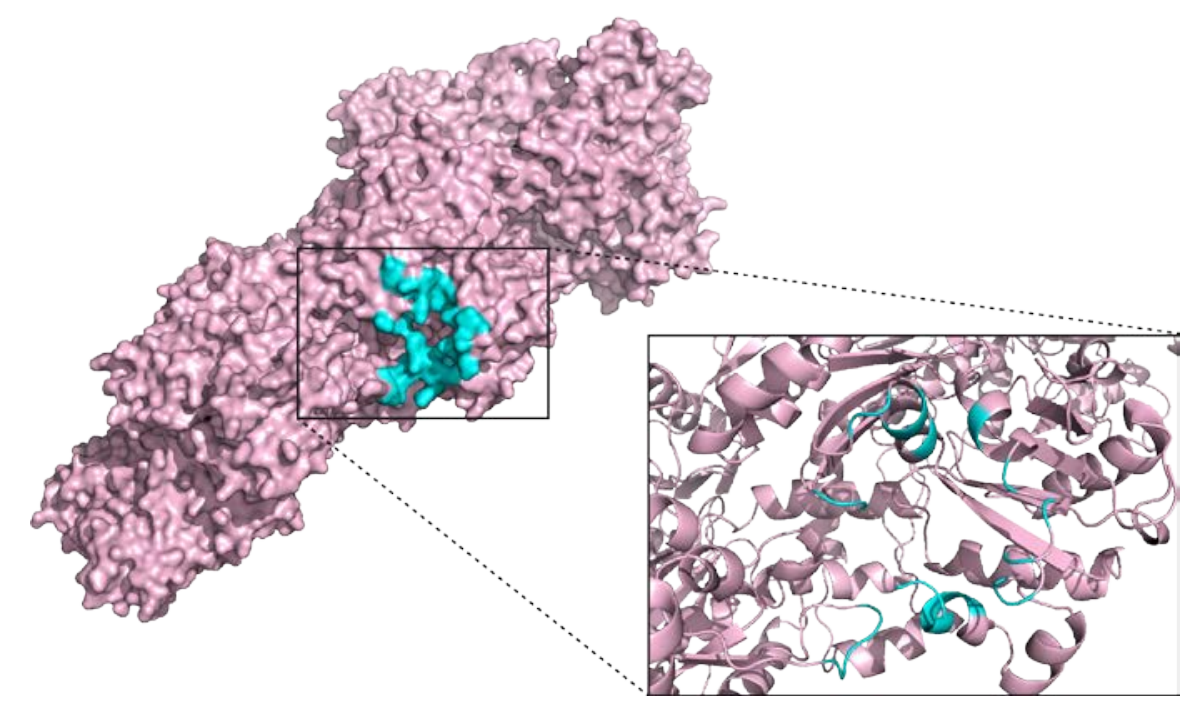

Figure 3.32. Active residues on F-actin used for docking calculation.

3D CryoEM-based structure of F-actin (pdb: 3J0S (Galkin, Orlova et al. 2011)). Active residues for docking calculation are represented in cyan color. 
Table 3.4. Statistic parameters from the lower score cluster from docking.

\begin{tabular}{|l|l|}
\hline HADDOCK score & $1.6 \pm 1.1$ \\
\hline Cluster size & 161 \\
\hline RMSD from the overall lowest-energy structure & $1.0 \pm 0.8$ \\
\hline Van der Waals energy & $-54.4 \pm 8.2$ \\
\hline Electrostatic energy & $-291.9 \pm 105.8$ \\
\hline Desolvation energy & $110.5 \pm 17.1$ \\
\hline Restraints violation energy & $38.8 \pm 8.07$ \\
\hline Buried Surface Area & $1823.0 \pm 96.1$ \\
\hline Z-Score & -1.5 \\
\hline
\end{tabular}

a

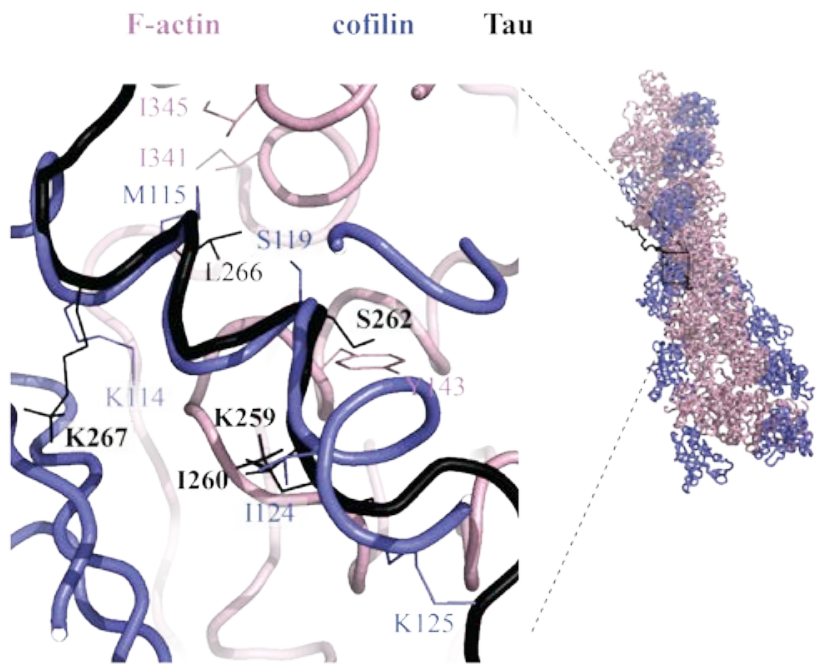

b

Cofilin

Tau (268-254)

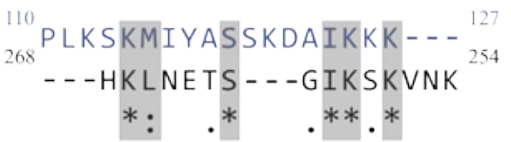

Figure 3.33. Superposition of 3D structures of both cofilin and Tau(250-290) in complex with Factin.

(a) Crystal structure of cofilin (blue) in complex with F-actin (pink; PDB id: 3J0S), superimposed onto the F-actin bound form of Tau(254-290) as derived from protein-protein docking (black). Side chains of selected residues are shown. (b) Sequence alignment of cofilin with residues 268-254 of Tau. 
Next, we compared the docking model of the Tau(254-290)/F-actin complex with the 3D structure of cofilin-decorated F-actin (Figure 3.33a). The comparison showed that the $\alpha$-helix of Tau(254-220 290) is positioned in the binding pocket in a very similar way as the recognition helix of cofilin (blue). L266 of Tau(254-290) is only $4 \AA$ away from I345 on F-actin, while the HE atom of the cofilin residue M115 is $3.1 \AA$ separated from the HG2 atom of I341 in actin. In addition, I260 of $\operatorname{Tau}(254-290)$ was found in a similar location as I124 from cofilin. In agreement with the structural similarity, the involved sequences from cofilin and Tau can be well aligned (Figure 3.33b). Optimal sequence alignment was achieved, when the sequence of Tau was inverted (Figure 3.33b), indicating an inverted orientation of its recognition helix when compared to cofilin (Figure 3.33a). 


\section{Discussion}

\subsection{The interaction of Tau with actin}

Tau and other microtubule-associated proteins, such as MAP2, not only bind to microtubules but also to filamentous actin, which results in cross-linking and bundling of actin filaments (Griffith and Pollard 1982, Selden and Pollard 1983, Sattilaro 1986, Yamauchi and Purich 1993, Cunningham, Leclerc et al. 1997, Biernat, Wu et al. 2002, He, Wang et al. 2009, Elie, Prezel et al. 2015). The interaction of Tau with actin is important for neurite outgrowth and synaptic dysfunction (Knops, Kosik et al. 1991, Biernat and Mandelkow 1999, Fulga, Elson-Schwab et al. 2007). Pathogenic forms of Tau have been connected to the formation of actin-rich rods (Whiteman, Gervasio et al. 2009), which were found to induce neurodegeneration in Drosophila neurons (Fulga, Elson-Schwab et al. 2007). In addition, Tau-induced neurotoxicity is associated with increased F-actin levels (Fulga, Elson-Schwab et al. 2007), and Tau-induced remodeling of the actin cytoskeleton can cause plasma membrane blebbing (Torres-Cruz, Rodriguez-Cruz et al. 2016).

G-actin form gels in presence of Tau (Yamauchi and Purich 1993), most probably because of a direct interaction between Tau and monomeric actin. In addition, preincubation of the 3-repeat isoform of Tau, Tau23, the proline-rich region as well as the MBD with skeletal or non-muscle actin promoted actin assembly (He, Wang et al. 2009). Actin monomers also inhibit the association of Tau with tubulin(Farias, Munoz et al. 2002).

G-actin binding proteins regulate the size, localization and dynamics of the large pool of unpolymerized actin in cells (Dominguez 2004). G-actin binding proteins include profilin, cofilin and formin, which interact with G-actin and regulate their incorporation into filament ends controlling actin dynamics in cells. Although Tau and Tau peptides bind to G-actin, they promote and not inhibit G-actin assembly, indicating that Tau cannot be considered as an actin sequestering protein. 


\subsubsection{Tau binds to G-actin}

To study the interaction between Tau and monomeric actin, we first determined the Tau/G-actin binding affinity through the use of NBD-labeled G-actin. The change in fluorescence of NBDlabeled actin has been broadly used for studying the interaction between actin and other actinbinding proteins (Detmers, Weber et al. 1981, Bryan and Kurth 1984, Hertzog, Yarmola et al. 2002, Ojala, Paavilainen et al. 2002, Mattila, Quintero-Monzon et al. 2004). We found that Tau binds to G-actin with an affinity of $0.091 \pm 0.007 \mu \mathrm{M}$ and decreased the NBD fluorescence in a $45 \%$. This result is in agreement to the reported by He, Wang et al (He, Wang et al. 2009) were a solid phase assay revealed an affinity of $0.029 \pm 0.005 \mu \mathrm{M}$ for the binding between Tau23 and $\beta / \gamma$-G-actin respectively. R2 domain in hTau40 is not present in Tau23 and therefore one would expect different affinities between both Tau isoforms. However, in a self-assembly test a 1.4 times more peptide from R2 domain (i.e. ${ }^{287}$ VQSKCGSKDNIKHVPGGG ${ }^{305}$ ) was required to promote a comparable assembly effect than a peptide from R1 domain (Moraga, Nunez et al. 1993). This means that $R 2$ has a lower contribution to the interaction than other Tau regions involved in binding and explains why the affinities between hTau40 and Tau23 are comparable.

We have determined that two fragments of Tau, R1 $\left({ }^{254}\right.$ KNVKSKIGSTENLKHQPGGG KVQIINKKLDL ${ }^{284}$ ) and K18 (repeat region of Tau) bind to G-actin with affinities $0.76 \pm 0.06$ $\mu \mathrm{M}$ and $0.21 \pm 0.03 \mu \mathrm{M}$ respectively. If affinities of hTau40, K18 and Tau(254-284) (Figure 3.1d-f ) are compared, it can be inferred that interaction of Tau and G-actin depends on the number of interacting residues. Interestingly a similar peptide from the MBD of MAP-2 and MAP-4 promoted bundles of F-actin (Moraga, Nunez et al. 1993).

\subsubsection{The MBD and proline-rich region of Tau interact with G-actin}

Evidences about the interaction between Tau23, the shortest Tau isoform in the human brain (see Figure 1.3), and G-actin had been previously reported (He, Wang et al. 2009). The authors showed, by using solid phase assay, that Tau23 binds to G-actin and promoted actin assembly being both the proline-rich and repeat regions responsible for the interaction. However both regions conserved the bundling effect of full-length Tau23.

Here we demonstrated, by means of NMR spectroscopy, that hTau40, the largest isoform of Tau, interacts with G-actin as well. In agreement with He, Wang et al. the study revealed that residues 
from both the proline-rich and the repeat regions of Tau are those in close contact with G-actin (Figure 3.3). If the repeat region on the intensity plot is analyzed binding hot spots separated by flexible linkers in the Tau sequence can be identified. Interestingly, residues from L114 to T123 in the region between N2 and P1 (see top panel on Figure 3.3), which has an alpha helical propensity (Mukrasch, Bibow et al. 2009), are also involved in binding to G-actin. In addition selected regions of K18, adopt $\alpha$-helical conformations upon binding to micelles (Barre and Eliezer 2013). All together suggests, in agreement with our finding, that G-actin might also recognize $\alpha$-helical structures of Tau.

\subsubsection{Different Tau motifs bind to the same region of G-actin}

Competition experiments between Tau and Tau peptides from the identified binding hot spots were performed to determine whether these Tau fragments bind to the same site on the actin surface. Peptides from the MBD (residues 254-290 and 292-319) as well as a peptide from the P2 region (residues 211-242) detached full-length Tau from the actin surface. This suggests that the three Tau peptides bind to a common region in actin where they interact in a competitive manner (Figure 4.1a). Other possibility would be a cooperative effect between the involved regions so that when one of them is affected the rest would feel the effect as a consequence.

That Tau binds to a single region on the G-actin surface by using distinct segments across the MBD opens the possibility that one molecule of Tau can cross-link at least two molecules of actin (Figure 4.1b). Two cross-linked molecules of actin may serve as a core to assemble new actin filaments. Unfortunately the fluorescence-binding assay does not consider stoichiometry bigger than one. However, this problem could be addressed by using Isothermal Calorimetric Titration (ITC). 
$\mathbf{a}$

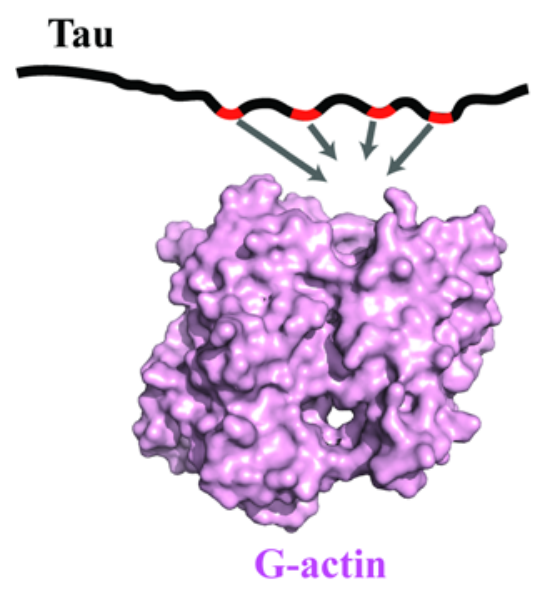

b

\section{Tau-driven actin polymerization}
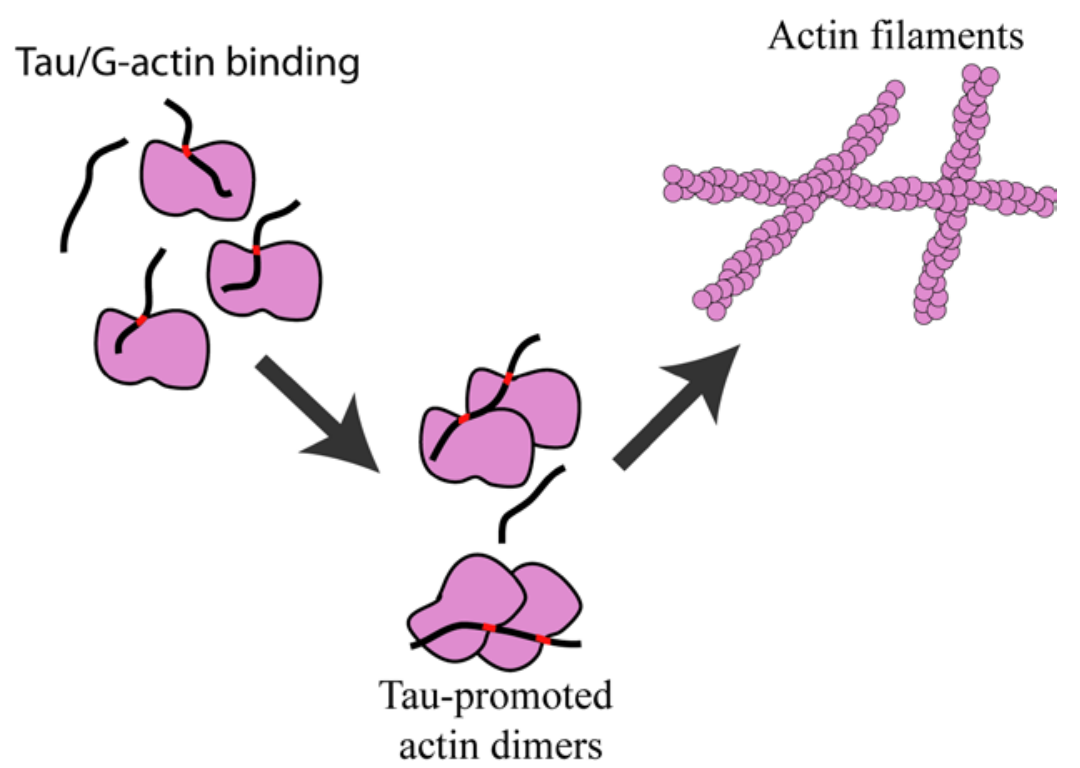

Figure 4.1. Pictorial representation of Tau/G-actin interaction.

(a) Tau (black line) interacts with G-actin (pink) by means of short regions (red lines) and recognizes a specific region on G-actin surface. (b) Cartoon representation of the mechanism through which Tau might bring actin monomer close each other promoting actin polymerization.

\subsubsection{Tau binds to the hydrophobic pocket of G-actin}

The dynamics and reorganization of actin filaments observed in motile cells requires the availability of actin monomers to be tightly regulated. This regulation is achieved by a group of highly conserved G-actin-binding proteins using different mechanisms of action (Lee and Galbraith 1992, Pollard and Borisy 2003, Dominguez and Holmes 2011). In motile cells the presence of a large pool of actin monomers is required to allow rapid filament extension. Monomer-sequestering proteins are those in charge of this job as a response to different signaling process.

Cibulout, a protein from Drosophila, is one of the proteins having a high sequence similarity with Thymosin- $\beta 4$, the major variant of the sequestering G-actin $\beta$-thymosins (Boquet, Boujemaa et al. 2000). However, in presence of caped actin, Cib has a profilin-like function participating in actin assembly at the barbed end (Pollard and Cooper 1984, Pring, Weber et al. 1992, Boquet, Boujemaa et al. 2000). As mentioned in section 1.4.3.1, Cib(D1), one of the three repeats of Cib binds to G-actin with an affinity of 2.5 $\mu \mathrm{M}$ (Boquet, Boujemaa et al. 2000, 
Hertzog, Yarmola et al. 2002). In addition, Cib adopts an $\alpha$-helical conformation upon binding to the hydrophobic pocket of actin (Hertzog, van Heijenoort et al. 2004).

To address whether Tau binds to the hydrophobic pocket of actin, we performed competition experiments between Tau and Cib(D1) in presence of G-actin. In this experiment G-actin was previously incubated with LatB, a drug that inhibits actin assembly. The experiment revealed that both Tau and Cib(D1) share the same binding site on actin. A 20-fold excess of Cib(D1) detached Tau from the actin surface (Figure 3.7), a result that was further confirmed by STD were short Tau fragments competed with Cib for binding to G-actin (Figure 3.8). The STD experiments also supported the hypothesis that short Tau peptides bind to the same region of Gactin as full-length Tau. In addition, competition experiments with LatB suggested that Tau does not bind to actin's nucleotide-binding pocket. Furthermore, PRE data confirmed not only that Tau binds to the hydrophobic pocket but also that the proline-rich and repeat regions are those in close contact with actin. These regions involve the hexapeptides ${ }^{275}$ VQIINK ${ }^{280}$ and ${ }^{306}$ VQIVYK $^{311}$, which are known to be prompt to Tau aggregation in AD (von Bergen, Friedhoff et al. 2000).

\subsubsection{Influence of phosphorylation of Tau at KXGS motifs on the interaction with G-actin}

Tau undergoes several post-translational modifications that influence its function, aggregation and toxicity (Gong, Liu et al. 2005). One of the most important post-translational modification is phosphorylation, because Tau is hyper-phosphorylated in the brains of patients with Alzheimer's disease (Bancher, Brunner et al. 1989). It has been reported that phosphorylation of serine residues at KXGS motifs in the repeat domain of Tau, such as S262, strongly reduce the affinity of Tau for microtubules (Biernat, Gustke et al. 1993). Here we demonstrated that phosphorylation of Tau(254-284) on S262 decreases the affinity of the peptide to G-actin (Figure 3.1c and g). Interestingly regions, which are in close proximity to G-actin, involve the KXGS motifs (Figure 3.9d) whose phosphorylation attenuates the interaction with actin. This suggests that the KXGS motifs of Tau should be involved in binding in both Tau/MT and Tau/actin interactions. Although we have said that the interaction between Tau and actin should have mainly a hydrophobic nature the existence of local electrostatic contribution cannot be discarded. If we assume that the interaction of the KXGS motifs of Tau are those having electrostatic 
contacts with actin we can argue that when serine is phosphorylated it acquires a negative charge generating an electrostatic repulsion in the binding site.

\subsection{Relevance of the interaction between Tau and G-actin}

Although the interaction between Tau and monomeric actin was already reported nothing was known about the mechanism of interaction. Here we reported not only a novel mechanism for the Tau/G-actin interaction but also the Tau residues involved in binding. So far several actinbinding proteins have been identified to bind at the hydrophobic pocket of actin adopting an $\alpha$ helical conformation upon binding (Dominguez 2004). The fact that Tau binds at this region, as suggested by competition with Cib and PRE data, could indicate that Tau might adopt alpha helical conformation as well. Furthermore selected residues in K19, a Tau construct including $\mathrm{R} 1, \mathrm{R} 3$ and R4 regions on the MBD, is known to adopt alpha helical conformation upon binding to micelles (Barre and Eliezer 2006). The Tau segments having an $\alpha$-helical conformation upon binding to micelles are L253-G261, L315-G323 and F346-G355. Interestingly these residues are also involved in binding to actin (see section 3.1.4.2.2). All together suggests that Tau could have an important role in the actin turnover, facilitating the actin polymerization and contributing to the dynamics of actin in cells. This conclusion further support the correlation between the loss of spines and the presence of Tau in dendrites in Alzheimer's disease (Zempel, Thies et al. 2010).We have shown that Tau phosphorylation decreases the affinity of the Tau/G-actin interaction. Furthermore Tau phosphorylation is associated with Tau aggregation in AD (Martin, Latypova et al. 2013). Therefore this could explain the correlation between Tau neurotoxicity and alterations of actin organization in animal models of Alzheimer's disease (Fulga, ElsonSchwab et al. 2007).

\subsection{Tau interacts with F-actin}

Misfolding and aberrant accumulation of the microtubule-associated protein Tau is a pathological hallmark of Alzheimer's disease (AD) (Williams 2006). Occurrence of Tau deposits correlates with the loss of cognitive functions in $\mathrm{AD}$, suggesting a connection between Tau and synaptic transmission (Giannakopoulos, Herrmann et al. 2003). Because Tau directly interacts with F-actin and regulate its stability (Yamauchi and Purich 1993, Fulga, Elson-Schwab et al. 
2007, He, Wang et al. 2009, Frandemiche, De Seranno et al. 2014), the interaction of Tau with Factin might influence synaptic plasticity (Hotulainen and Hoogenraad 2010). In agreement with the importance of the Tau/F-actin interaction, actin-rich paracrystalline inclusions, so-called Hirano bodies, are found in brain histopathological samples of $\mathrm{AD}$ and related tauopathies (Hirano 1994, Makioka, Yamazaki et al. 2014). In addition, it was recently shown that acetylation of Tau modulates actin polymerization and enhances synaptic dysfunction (Tracy, Sohn et al. 2016).

The human central nervous system contains six different isoforms of Tau, which differ in the number of N-terminal inserts and have either three or four imperfect repeats in their C-terminal half (Figure 1.3) (Weingarten, Lockwood et al. 1975). The repeats are important for binding of Tau to microtubules and F-actin (Butner and Kirschner 1991, Gustke, Trinczek et al. 1994). Other microtubule-associated proteins from the MAP2 family also bind to actin filaments through their repeat domain (Griffith and Pollard 1982). In addition, certain fragments of the repeat-domain of Tau are able to bind actin and promote actin bundling (Moraga, Nunez et al. 1993).

In agreement with previous findings, we show that Tau binds with high-affinity to filamentous actin, resulting in F-actin bundling. Our results support the previous reports about the interaction between Tau and F-actin and demonstrate that specific regions inside the Tau sequence are the responsible for this interaction. The proline-rich and repeat regions are involved in binding, an observation that is in agreement with previous reports. Interaction with the proline-rich region is primarily of electrostatic nature. In contrast, short hydrophobic residue stretches in the repeat domain bind to the hydrophobic pocket between subdomain 1 and 3 of actin (Figure 3.18b).

In agreement with Correas, Padilla et al. our result showed that short Tau motives like Tau(254290) and Tau(292-319) are able to bundle F-actin while a shorter construct having only 15 residues like Tau(254-268) failed in bundling actin filaments (Figure 3.14).

\subsection{Tau binds to F-actin in a similar fashion than to G-actin: as seen by NMR}

If the intensity plots and affinities from Tau/G-actin and Tau/F-actin interactions are compared is clear that Tau binds similarly in both cases to G- or F-actin. Thus it is reasonable to think that 
Tau binds also on the hydrophobic pocket of F-actin, which is exposed to the solvent in the filamentous form of actin (Dominguez 2004, Dominguez and Holmes 2011). NMR-based competition experiments and co-sedimentation assays with cofilin in presence of Tau and F-actin confirmed this hypothesis. On the other hand PRE data revealed six defined peaks corresponding to residues K243-V246, K259-K268, Q276-K290, S305-Y310, S320-H330 and K375-F378 are involved in binding (Figure 3.18b), which are nearly the same in case of PRE data from G-actin. The F-actin-interacting Tau residues as in case of the interaction with G-actin are separated from each other by flexible linkers, which enable a high degree of dynamics and multivalency in the Tau/F-actin interaction.

The fact that Tau binds on the same place than cofilin which at the same time share the binding site with gelsolin (Mclaughlin, Gooch et al. 1993) may suggest that these proteins have similar functions. Nevertheless the binding of Tau to monomeric actin suggests that Tau might have also a profilin-like function as mediator of the actin assembly.

We further investigated the nature of the interaction between Tau and F-actin using a NMRbased salt titration experiment (Figure 3.19a). The experiment revealed that even at high salt concentration, regions form the repeat and P2 regions of Tau were still bound to F-actin in agreement to reported in 2009 (He, Wang et al. 2009). This is a surprise if we consider the fact that Tau binds on the hydrophobic pocket of actin where hydrophobic interaction should command. The fact that this interaction has an important hydrophobic contribution could be particularly important in cell were physiological conditions can minimize electrostatic interactions.

\subsection{Phosphorylation of Tau affects its binding to F-actin}

Tau undergoes several post-translational modifications that influence its function, aggregation and toxicity (Gong, Liu et al. 2005). An important post-translational modification is phosphorylation, because Tau is hyper-phosphorylated in the brains of patients with Alzheimer's disease (Bancher, Brunner et al. 1989). Phosphorylation of serine residues at KXGS motifs in the repeat domain of Tau, such as S262, strongly reduce the affinity of Tau for microtubules (Biernat, Gustke et al. 1993). In addition, phosphorylation by endogenous protein kinases inhibits the ability of Tau and MAP2 to cross-link and bundle actin filaments (Selden and Pollard 1983, Sattilaro 1986). Consistent with these data, we showed that phosphorylation of Tau by 
MARK2 reduces the affinity of Tau for F-actin (Figure 3.20). In addition, specific phosphorylation at S262 decreased the F-actin affinity of Tau(254-284) (Figure 3.21).

While our as well as previous biophysical studies demonstrated that phosphorylation at the KXGS motifs decreases the affinity of Tau for both microtubules and F-actin, cellular studies showed that phosphorylation of the KXGS motifs cause MAP-2 and Tau to localize to the actin cytoskeleton (Ozer and Halpain 2000, Biernat, Wu et al. 2002). In addition, phosphorylated Tau is recruited to actin-cofilin rods, which might lead to neuropil threads (Whiteman, Gervasio et al. 2009). The combined data suggest that phosphorylation of Tau at KXGS motifs attenuates the direct interaction with microtubules and F-actin, but fosters the interaction with actin-containing structures, in which other proteins such as cofilin, might mediate the interaction with phosphorylated Tau.

\subsection{Insights into the structure of Tau bound to F-actin}

Microtubule-associated proteins (MAPs) bind to, stabilize and promote assembly of microtubules (Lee 1993, Mandelkow and Mandelkow 1995). In addition, MAPs bundle actin filaments and cross-link the cellular cytoskeleton formed by microtubules and actin filaments (Figure 4.2) (Griffith and Pollard 1982, Selden and Pollard 1983, Sattilaro 1986, Yamauchi and Purich 1993, Biernat, Wu et al. 2002, He, Wang et al. 2009). The interaction of MAPs with actin is important for neurite outgrowth (Knops, Kosik et al. 1991, Biernat and Mandelkow 1999). Representative MAPs are MAP1a, MAP1b, MAP2a, MAP2b, MAP2c, MAP4 and Tau, and isoforms of these proteins, which are often generated by alternative splicing (Lee 1993).

As described on section 1.3.1 Tau occurs in six different isoforms in the human central nervous system (Butner and Kirschner 1991, Gustke, Trinczek et al. 1994). The Tau isoforms differ in the number of N-terminal inserts and have either three or four imperfect repeats in their C-terminal half (Weingarten, Lockwood et al. 1975). The imperfect repeats are important for binding to both microtubules and actin. In addition, short fragments of the microtubule-binding domain of Tau promote actin bundling (Moraga, Nunez et al. 1993). Proteins from the MAP-2 family also bind to actin filaments through their repeat domain (Griffith and Pollard 1982). Because a single repeat interacts with both monomeric and filamentous actin, but does not bundle actin filaments, more than one microtubule-binding repeat is believed to be required for bundling of filamentous actin (Correas, Padilla et al. 1990). 


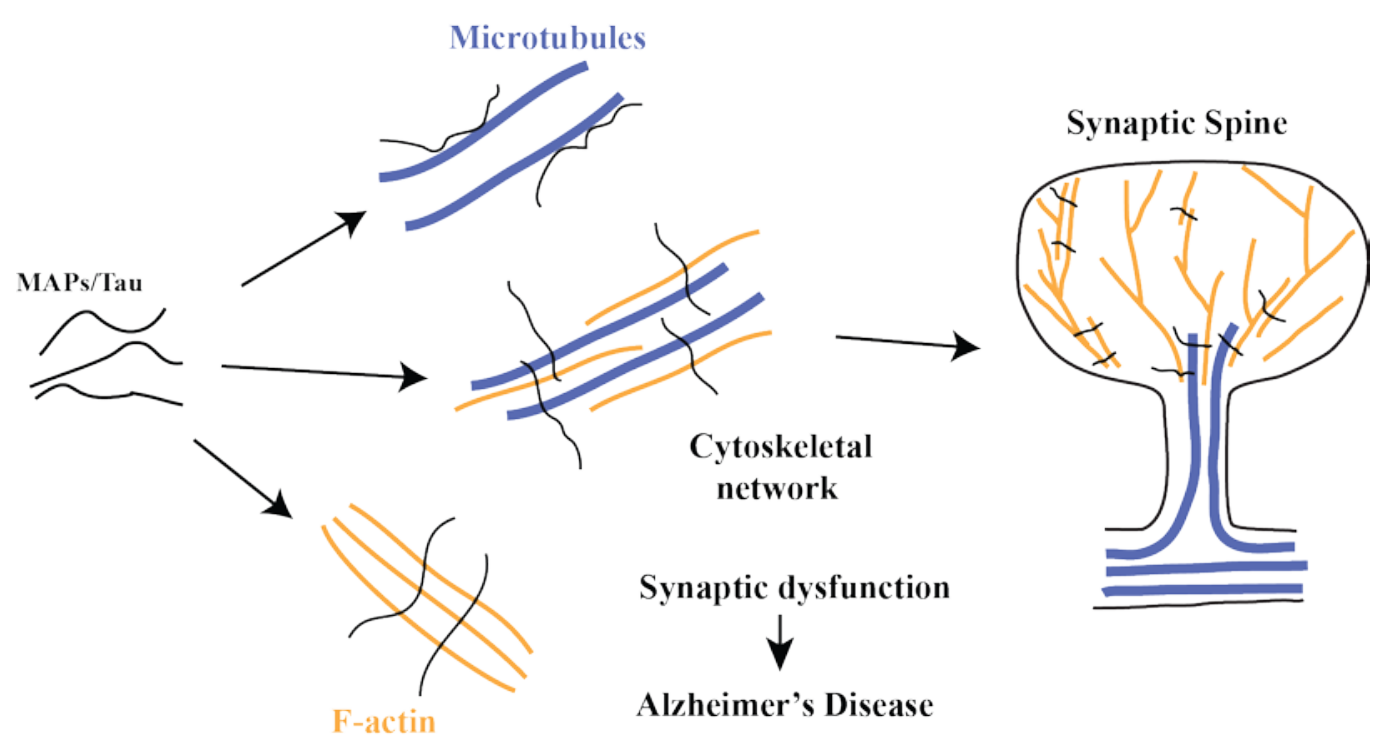

Figure 4.2. Schematic representation of the importance of MAPs for the cellular cytoskeleton.

MAPs (black) regulate microtubule dynamics (blue), bundle actin filaments (yellow) and cross-link actin filaments and microtubules. Aberrant interaction of Tau with F-actin is associated with synaptic dysfunction in Alzheimer's disease.

As Tau is intrinsically disordered in solution the knowledge of its 3D structure upon binding to actin is primordial to understand the mechanism of the interaction and its function in terms of Factin stabilization. We are particularly interested how Tau promotes the actin assembly and cytoskeletal network stability. Extracting structural information from the Tau/F-actin complex, which has a size on MDa order, is challenging because of the size limitation of NMR spectroscopy. Nevertheless, we have already shown that Tau bind to G- and F-actin by means of defined hot spots separated by flexible linkers. Based on this finding we can use the so-called “divide and conquer strategy” to derive the structure of Tau bound to F-actin.

If the ligand is weakly bound and there exists a fast exchange between free and bound forms of the ligand, detailed structural and dynamic information can be obtained by using tr-NOE and STD measurements (sections 2.10.2 and 2.10.5). STD experiment revealed that short Tau peptides are able to bind F-actin in a micro to millimolar range (Figure 3.22). The observation of a STD effect for peptides like Tau(254-268), Tau(254-290) and Tau(292-319) suggests that these peptides are suitable subjects for structure calculation using the tr-NOE approach. 


\subsubsection{Tau peptides recognizes F-actin by means of an alpha helical motifs}

We further showed that the F-actin-interacting residues in the repeat domain of Tau, fold into short $\alpha$-helices upon binding to filamentous actin (Figure 3.26, Figure 3.29 and Figure 3.31). Folding of local regions of Tau into $\alpha$-helical structure is consistent with the formation of $\alpha$ helices in actin-binding proteins, in which the actin-interacting region is intrinsically disordered prior to binding to actin (Dominguez 2004, Dominguez and Holmes 2011). The Tau regions that fold into $\alpha$-helical structure include the two hexapeptides, ${ }^{275} \mathrm{VQIINK}^{280}$ and ${ }^{306} \mathrm{VQIVYK}^{311}$, at the beginning of repeats R2 and R3 (Figure 3.29b and Figure 3.31). The two hexapeptides are important for aberrant aggregation of Tau into paired helical filaments (von Bergen, Friedhoff et al. 2000). In solution, the two hexapeptides populate extended structure (Mukrasch, Bibow et al. 2009), are found in cross- $\beta$ structure in amyloid fibrils (Andronesi, von Bergen et al. 2008, Daebel, Chinnathambi et al. 2012), but fold into $\alpha$-helical structure in complex with F-actin (Fig. 3.29). In addition to the two hexapeptides, the N-terminal halves of each of Tau's four 18-residue aminoacid repeats (Goode and Feinstein 1994) bind to F-actin's hydrophobic pocket and fold into $\alpha$-helix (Figure 3.29). Consistent with the compatibility of these regions for $\alpha$-helical conformation, Tau residues 253-260, 315-322 and 345-354 populate helical structure in the presence of detergent and upon binding to membranes (Barre and Eliezer 2006). Docking of experimentally determined Tau helices to the F-actin surface together with sequence alignment showed that the overall position of the interacting Tau and cofilin helices is well conserved, but the directionality of their polypeptide chain is inverted (Figure 3.33). The directionality of the Tau(259-267)-helix is similar to that of the actin interacting helix of toxofilin, an actin-binding protein that is secreted into host cells during invasion (Dominguez and Holmes 2011). The KXGS motif, which contains S262, is located in one of the F-actin interacting helices of Tau (Figure 3.29a). The side chain of S262 points towards actin's hydrophobic binding pocket (Figure 3.33a), providing a structural explanation for the lower affinity of S262-phosphorylated Tau(254-284). S262 may share hydrogen bound with Y143 in actin because they are only $1.6 \AA$ away in the docked structure (Figure 3.33a), nevertheless this interaction may be affected when the side chain of serine get phosphorylated. Taken together the data show that Tau utilizes a structural mechanism for binding to F-actin that is used by several actin-binding proteins. 


\subsubsection{Rolle of Tau in the cytoskeletal network organization}

Binding of Tau and MAP2 to filamentous actin results in actin cross-linking and filament bundling (Selden and Pollard 1983, Sattilaro 1986, Butner and Kirschner 1991, Wang, GrundkeIqbal et al. 1996, Dominguez, Boelens et al. 2003, Hotulainen and Hoogenraad 2010, Kadavath, Jaremko et al. 2015). At the same time, binding to F-actin can be retained, but the cross-linking activity disrupted. For example, a C-terminal fragment and a peptide corresponding to the second microtubule-binding repeat bind actin, but are incapable of mediating actin cross-linking (Sattilaro 1986, Correas, Padilla et al. 1990). These findings suggest that multiple domains of Tau might be required for actin cross-linking. Consistent with this hypothesis, we showed that a single Tau helix is sufficient for binding to F-actin, but at least two helices, which are separated by a flexible linker, are required for cross-linking activity (Figure 3.14d and Figure 3.26). On this basis, we developed a model, in which two helical regions in Tau are bound to two different actin filaments (Figure 4.3). Because Tau contains seven actin-binding segments (Figure 3.18), up to seven actin filaments could be linked together by a single Tau molecule.

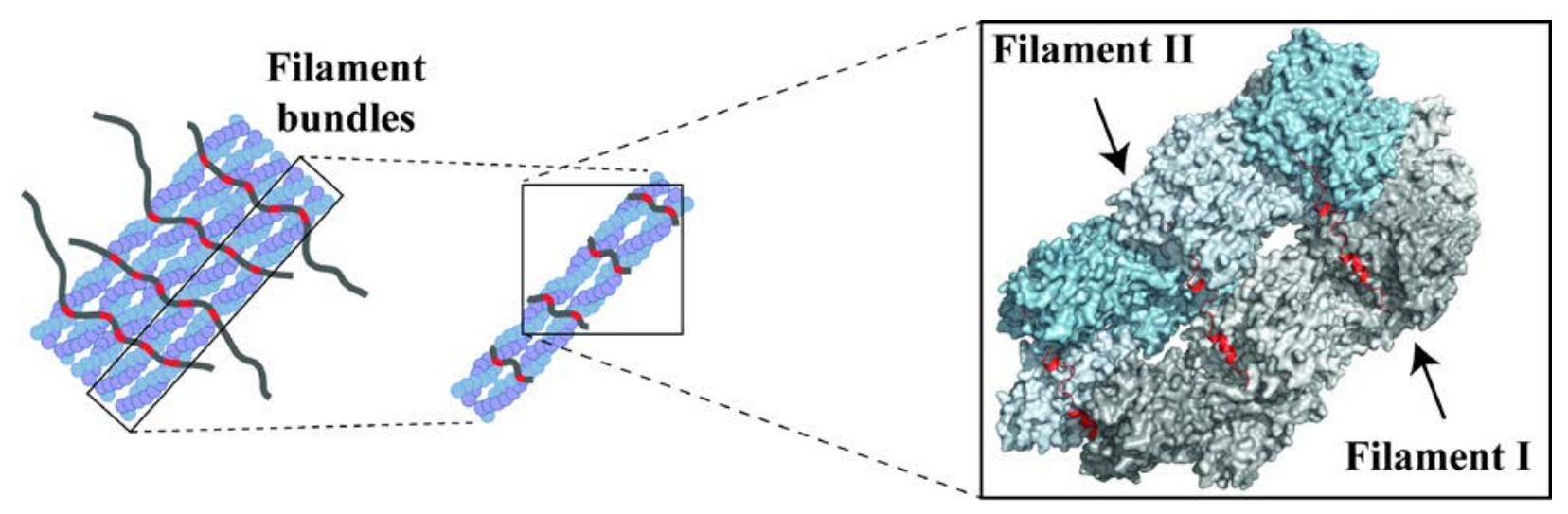

Figure 4.3. Mechanistic model of the Tau-induced F-actin bundles.

Schematic representation of cross-linking of actin filaments by $\boldsymbol{\alpha}$-helices of Tau, which are separated by flexible linkers. Each $\boldsymbol{\alpha}$-helix (shown in red) is bound to a hydrophobic actin pocket in a neighboring filament (grey and light blue). 


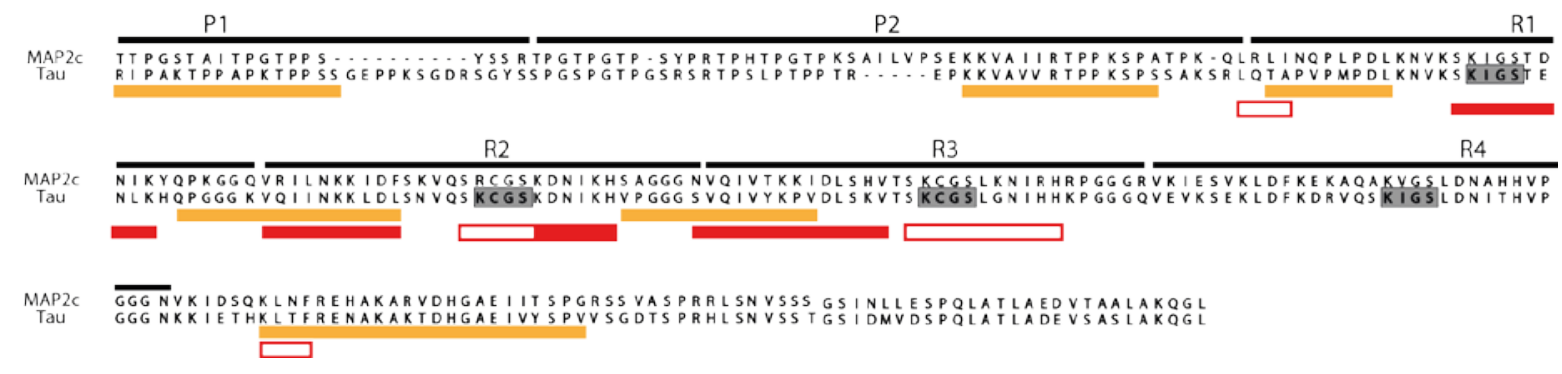

Figure 4.4. Sequence alignment of the repeat domains of MAP2c and Tau.

Tau residues, which bind to F-actin, are marked by red open bars, experimentally proven F-actin induced helical structure by red filled bars, and microtubule-binding sites in orange. KXGS motifs are highlighted.

Steric constraints are minimized by the presence of flexible linkers between the seven actinbinding segments. In addition, the excess positive charge, which is present in the repeat domain of Tau, will decrease electrostatic repulsion between the negatively charged filament surfaces. Because of the high sequence conservation in the repeat domain between Tau and other microtubule-associated proteins such as MAP2c (Figure 4.4), our data suggest that the described mechanism for actin cross-linking is more widely applicable.

The interaction between actin filaments and microtubules is important for cellular processes including cell division, vesicle and organelle transport, axonal growth and migration (Rodriguez, Schaefer et al. 2003). Tau mediates interactions between microtubules and actin in neuronal growth cones, where Tau-mediated cytoskeletal interactions promote morphological changes. In vitro, Tau binds simultaneously to microtubules and actin filaments (Elie, Prezel et al. 2015). We previously showed that the Tau residues 168-185, 224-237, 245-253, 269-284, 300-313, and 375-398 bind to microtubules at the interface between tubulin heterodimers (Figure 4.4) (Kadavath, Hofele et al. 2015). Thus, when both F-actin and microtubules are present, they compete for binding to residues 245-246, 275-284 and 305-313, while Tau residues 259-267, 289-297 and 320-330 will preferentially interact with F-actin (Figure 4.4). Notably, the conformations that are induced in Tau upon binding to F-actin and microtubules are very different, because F-actin interaction is connected to helix formation (Figure 3.29), while short Tau regions fold into a hairpin-like structure in complex with microtubules (Kadavath, Jaremko et al. 2015). Thus, the availability of multiple Tau sites for binding to microtubules and F-actin together with Tau residues, which are dedicated for binding to only F-actin or microtubules (Figure 4.4), enables multivalent cross-linking of microtubules and actin filaments (Figure 4.5). 


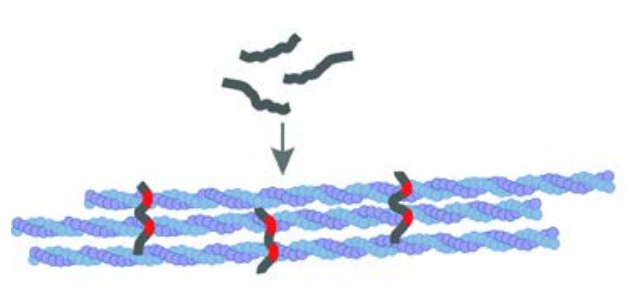

Tau

1 a-helix

- MT-binding motif
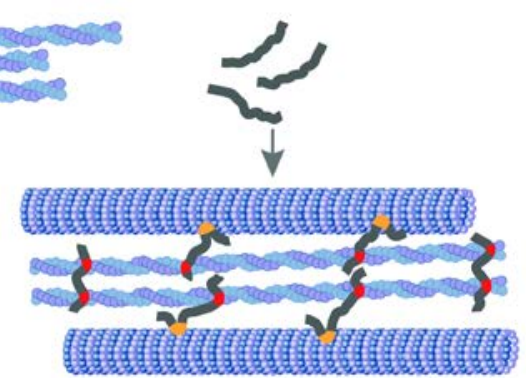

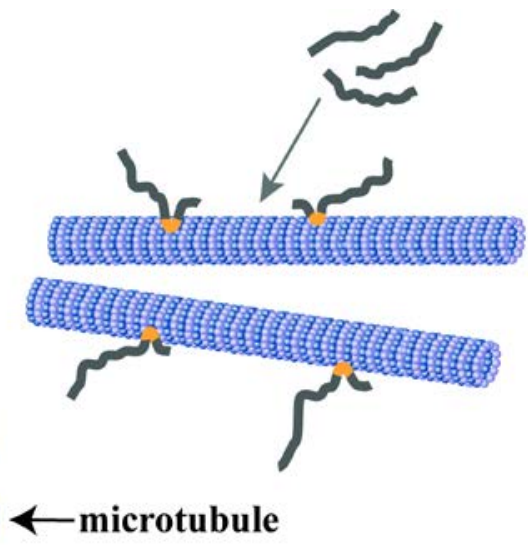

Figure 4.5. Mechanistic model of Tau-mediated F-actin/microtubule network formation.

Because Tau contains multiple interaction sites for both F-actin (this work) and microtubules, Tau can cross-link the cellular cytoskeleton. While short segments in the repeat domain fold into $\boldsymbol{\alpha}$-helical structure (red) in complex with F-actin, Tau residues in complex with microtubules form a microtubulespecific hairpin structure (orange). 


\section{References}

Aisen, P. S., J. Cummings and L. S. Schneider (2012). "Symptomatic and nonamyloid/tau based pharmacologic treatment for Alzheimer disease." Cold Spring Harb Perspect Med 2(3): a006395.

Al-Bassam, J., R. S. Ozer, D. Safer, S. Halpain and R. A. Milligan (2002). "MAP2 and tau bind longitudinally along the outer ridges of microtubule protofilaments." Journal of Cell Biology 157(7): 1187-1196.

Allingham, J. S., V. A. Klenchin and I. Rayment (2006). "Actin-targeting natural products: structures, properties and mechanisms of action." Cell Mol Life Sci 63(18): 2119-2134.

Allingham, J. S., C. O. Miles and I. Rayment (2007). "A structural basis for regulation of actin polymerization by pectenotoxins." J Mol Biol 371(4): 959-970.

Alonso, A. D., C. Beharry, C. P. Corbo and L. S. Cohen (2016). "Molecular mechanism of prionlike tau-induced neurodegeneration." Alzheimer's \& Dementia 12(10): 10901097.

Amniai, L., P. Barbier, A. Sillen, J. M. Wieruszeski, V. Peyrot, G. Lippens and I. Landrieu (2009). "Alzheimer disease specific phosphoepitopes of Tau interfere with assembly of tubulin but not binding to microtubules." Faseb Journal 23(4): 11461152.

Anand, R., K. D. Gill and A. A. Mahdi (2014). "Therapeutics of Alzheimer's disease: Past, present and future." Neuropharmacology 76: 27-50.

Andrianantoandro, E. and T. D. Pollard (2006). "Mechanism of actin filament turnover by severing and nucleation at different concentrations of ADF/cofilin." Molecular Cell 24(1): 13-23.

Andronesi, O. C., M. von Bergen, J. Biernat, K. Seidel, C. Griesinger, E. Mandelkow and M. Baldus (2008). "Characterization of Alzheimer's-like paired helical filaments from the core domain of tau protein using solid-state NMR spectroscopy." J Am Chem Soc 130(18): 5922-5928.

Balaram, P., A. A. Bothner-By and J. Dadok (1972). "Negative nuclear Overhuaser effects as probes of macromolecular structure." Journal of the American Chemical Society 94(11): 4015-4017.

Bancher, C., C. Brunner, H. Lassmann, H. Budka, K. Jellinger, G. Wiche, F. Seitelberger, I. Grundke-Iqbal, K. Iqbal and H. M. Wisniewski (1989). "Accumulation of abnormally phosphorylated tau precedes the formation of neurofibrillary tangles in Alzheimer's disease." Brain Res 477(1-2): 90-99.

Barre, P. and D. Eliezer (2006). "Folding of the repeat domain of tau upon binding to lipid surfaces." Journal of Molecular Biology 362(2): 312-326.

Barre, P. and D. Eliezer (2013). "Structural transitions in tau k18 on micelle binding suggest a hierarchy in the efficacy of individual microtubule-binding repeats in filament nucleation." Protein Sci 22(8): 1037-1048. 
Bax, A. and D. G. Davis (1985). "MLEV-17-based two-dimensional homonuclear magnetization transfer spectroscopy." Journal of Magnetic Resonance (1969) 65(2): 355-360.

Bernstein, B. W. and J. R. Bamburg (2010). "ADF/cofilin: a functional node in cell biology." Trends Cell Biol 20(4): 187-195.

Berriman, J., L. C. Serpell, K. A. Oberg, A. L. Fink, M. Goedert and R. A. Crowther (2003). "Tau filaments from human brain and from in vitro assembly of recombinant protein show cross-beta structure." Proc Natl Acad Sci U S A 100(15): 90349038.

Bertrand, J., V. Plouffe, P. Senechal and N. Leclerc (2010). "The Pattern of Human Tau Phosphorylation Is the Result of Priming and Feedback Events in Primary Hippocampal Neurons." Neuroscience 168(2): 323-334.

Bhattacharya, A., R. Tejero and G. T. Montelione (2007). "Evaluating protein structures determined by structural genomics consortia." Proteins 66(4): 778-795.

Biernat, J., N. Gustke, G. Drewes, E. M. Mandelkow and E. Mandelkow (1993). "Phosphorylation of Ser262 strongly reduces binding of tau to microtubules: distinction between PHF-like immunoreactivity and microtubule binding." Neuron 11(1): 153-163.

Biernat, J., N. Gustke, G. Drewes, E. M. Mandelkow and E. Mandelkow (1993). "Phosphorylation of Ser(262) Strongly Reduces Binding of Tau-Protein to Microtubules - Distinction between Phf-Like Immunoreactivity and Microtubule-Binding." Neuron 11(1): 153-163.

Biernat, J. and E. M. Mandelkow (1999). "The development of cell processes induced by tau protein requires phosphorylation of serine 262 and 356 in the repeat domain and is inhibited by phosphorylation in the proline-rich domains." Mol Biol Cell 10(3): 727-740.

Biernat, J., Y. Z. Wu, T. Timm, Q. Zheng-Fischhofer, E. Mandelkow, L. Meijer and E. M. Mandelkow (2002). "Protein kinase MARK/PAR-1 is required for neurite outgrowth and establishment of neuronal polarity." Mol Biol Cell 13(11): 40134028.

Blanchoin, L. and T. D. Pollard (1999). "Mechanism of interaction of Acanthamoeba actophorin (ADF/cofilin) with actin filaments." Journal of Biological Chemistry 274(22): 15538-15546.

Bobkov, A. A., A. Muhlrad, K. Kokabi, S. Vorobiev, S. C. Almo and E. Reisler (2002). "Structural effects of cofilin on longitudinal contacts in F-actin." Journal of Molecular Biology 323(4): 739-750.

Bodenhausen, G. and D. J. Ruben (1980). "Natural abundance nitrogen-15 NMR by enhanced heteronuclear spectroscopy." Chemical Physics Letters 69(1): 185-189.

Boquet, I., R. Boujemaa, M. F. Carlier and T. Preat (2000). "Ciboulot regulates actin assembly during Drosophila brain metamorphosis." Cell 102(6): 797-808.

Braunschweiler, L. and R. R. Ernst (1983). "Coherence transfer by isotropic mixing: Application to proton correlation spectroscopy." Journal of Magnetic Resonance (1969) 53(3): 521-528. 
Bravo-Cordero, J. J., M. A. O. Magalhaes, R. J. Eddy, L. Hodgson and J. Condeelis (2013). "Functions of cofilin in cell locomotion and invasion." Nature Reviews Molecular Cell Biology 14(7): 405-415.

Bryan, J. and M. C. Kurth (1984). "Actin-Gelsolin Interactions - Evidence for 2 Actin-Binding Sites." Journal of Biological Chemistry 259(12): 7480-7487.

Bubb, M. R., I. Spector, B. B. Beyer and K. M. Fosen (2000). "Effects of jasplakinolide on the kinetics of actin polymerization - An explanation for certain in vivo observations." Journal of Biological Chemistry 275(7): 5163-5170.

Bugyi, B. and M. F. Carlier (2010). "Control of Actin Filament Treadmilling in Cell Motility." Annual Review of Biophysics, Vol 39 39: 449-470.

Burtnick, L. D., E. K. Koepf, J. Grimes, E. Y. Jones, D. I. Stuart, P. J. McLaughlin and R. C. Robinson (1997). "The crystal structure of plasma gelsolin: implications for actin severing, capping, and nucleation." Cell 90(4): 661-670.

Butner, K. A. and M. W. Kirschner (1991). "Tau protein binds to microtubules through a flexible array of distributed weak sites." J Cell Biol 115(3): 717-730.

Butner, K. A. and M. W. Kirschner (1991). "Tau-Protein Binds to Microtubules through a Flexible Array of Distributed Weak Sites." Journal of Cell Biology 115(3): 717730.

Calabrese, B., M. S. Wilson and S. Halpain (2006). "Development and regulation of dendritic spine synapses." Physiology 21: 38-47.

Carlier, M. F. and D. Pantaloni (1997). "Control of actin dynamics in cell motility." Journal of Molecular Biology 269(4): 459-467.

Carlomagno, T. (2005). "Ligand-target interactions: what can we learn from NMR?" Annu Rev Biophys Biomol Struct 34: 245-266.

Cassimeris, L. and C. Spittle (2001). "Regulation of microtubule-associated proteins." International Review of Cytology - a Survey of Cell Biology, Vol 210 210: 163226.

Cavanagh, J., W. J. Fairbrother, A. G. Palmer Iii, M. Rance and N. J. Skelton (2007). CHAPTER 5 - RELAXATION AND DYNAMIC PROCESSES. Protein NMR Spectroscopy (Second Edition). Burlington, Academic Press: 333-404.

Cavanagh, J., W. J. Fairbrother, A. G. Palmer Iii, M. Rance and N. J. Skelton (2007). CHAPTER 6 - EXPERIMENTAL 1H NMR METHODS. Protein NMR Spectroscopy (Second Edition). Burlington, Academic Press: 405-532.

Chen, J., Y. Kanai, N. J. Cowan and N. Hirokawa (1992). "Projection Domains of Map2 and Tau Determine Spacings between Microtubules in Dendrites and Axons." Nature 360(6405): 674-676.

Chereau, D., F. Kerff, P. Graceffa, Z. Grabarek, K. Langsetmo and R. Dominguez (2005). "Actin-bound structures of Wiskott-Aldrich syndrome protein (WASP)homology domain 2 and the implications for filament assembly." Proceedings of the National Academy of Sciences of the United States of America 102(46): 16644-16649. 
Cho, J. H. and G. V. W. Johnson (2004). "Primed phosphorylation of tau at Thr231 by glycogen synthase kinase 3 beta (GSK3 beta) plays a critical role in regulating tau's ability to bind and stabilize microtubules." Journal of Neurochemistry 88(2): 349-358.

Churcher, I. (2006). "Tau therapeutic strategies for the treatment of Alzheimer's disease." Curr Top Med Chem 6(6): 579-595.

Cleveland, D. W., S. Y. Hwo and M. W. Kirschner (1977). "Physical and Chemical Properties of Purified Tau Factor and Role of Tau in Microtubule Assembly." Journal of Molecular Biology 116(2): 227-247.

Clore, G. M. and A. M. Gronenborn (1982). "Theory and Applications of the Transferred Nuclear Overhauser Effect to the Study of the Conformations of Small Ligands Bound to Proteins." Journal of Magnetic Resonance 48(3): 402-417.

Cohen, T. J., J. L. Guo, D. E. Hurtado, L. K. Kwong, I. P. Mills, J. Q. Trojanowski and V. M. Y. Lee (2011). "The acetylation of tau inhibits its function and promotes pathological tau aggregation." Nature Communications 2.

Cooper, G. M. (2000). Structure and Organization of Actin Filaments. Sunderland (MA): Sinauer Associates.

Cooper, J. A. (1987). "Effects of Cytochalasin and Phalloidin on Actin." Journal of Cell Biology 105(4): 1473-1478.

Correas, I., J. Diaz-Nido and J. Avila (1992). "Microtubule-associated protein tau is phosphorylated by protein kinase $\mathrm{C}$ on its tubulin binding domain." J Biol Chem 267(22): 15721-15728.

Correas, I., R. Padilla and J. Avila (1990). "The tubulin-binding sequence of brain microtubuleassociated proteins, tau and MAP-2, is also involved in actin binding." Biochem J 269(1): 61-64.

Cunningham, C. C., N. Leclerc, L. A. Flanagan, M. Lu, P. A. Janmey and K. S. Kosik (1997). "Microtubule-associated protein 2c reorganizes both microtubules and microfilaments into distinct cytological structures in an actin-binding protein280-deficient melanoma cell line." J Cell Biol 136(4): 845-857.

Daebel, V., S. Chinnathambi, J. Biernat, M. Schwalbe, B. Habenstein, A. Loquet, E. Akoury, K. Tepper, H. Muller, M. Baldus, C. Griesinger, M. Zweckstetter, E. Mandelkow, V. Vijayan and A. Lange (2012). "beta-Sheet core of tau paired helical filaments revealed by solid-state NMR." J Am Chem Soc 134(34): 13982-13989.

Daebel, V., S. Chinnathambi, J. Biernat, M. Schwalbe, B. Habenstein, A. Loquet, E. Akoury, K. Tepper, H. Muller, M. Baldus, C. Griesinger, M. Zweckstetter, E. Mandelkow, V. Vijayan and A. Lange (2012). "beta-Sheet Core of Tau Paired Helical Filaments Revealed by Solid-State NMR." Journal of the American Chemical Society 134(34): 13982-13989.

De Marino, S., C. Festa, M. V. D'Auria, T. Cresteil, C. Debitus and A. Zampella (2011). "Swinholide J, a potent cytotoxin from the marine sponge Theonella swinhoei." Mar Drugs 9(6): 1133-1141. 
Delaglio, F., S. Grzesiek, G. W. Vuister, G. Zhu, J. Pfeifer and A. Bax (1995). "Nmrpipe - a Multidimensional Spectral Processing System Based on Unix Pipes." Journal of Biomolecular Nmr 6(3): 277-293.

Detmers, P., A. Weber, M. Elzinga and R. E. Stephens (1981). "7-Chloro-4-nitrobenzeno-2-oxa1,3-diazole actin as a probe for actin polymerization." J Biol Chem 256(1): 99105.

Didry, D., F. X. Cantrelle, C. Husson, P. Roblin, A. M. E. Moorthy, J. Perez, C. Le Clainche, M. Hertzog, E. Guittet, M. F. Carlier, C. van Heijenoort and L. Renault (2012). "How a single residue in individual $\beta$-thymosin/WH2 domains controls their functions in actin assembly." The EMBO Journal 31(4): 1000-1013.

Dixit, R., J. L. Ross, Y. E. Goldman and E. L. Holzbaur (2008). "Differential regulation of dynein and kinesin motor proteins by tau." Science 319(5866): 1086-1089.

Dominguez, C., R. Boelens and A. M. Bonvin (2003). "HADDOCK: a protein-protein docking approach based on biochemical or biophysical information." J Am Chem Soc 125(7): 1731-1737.

Dominguez, R. (2004). "Actin-binding proteins - a unifying hypothesis." Trends in Biochemical Sciences 29(11): 572-578.

Dominguez, R. and K. C. Holmes (2011). "Actin Structure and Function." Annual Review of Biophysics, Vol 40 40: 169-186.

dos Remedios, C. G., D. Chhabra, M. Kekic, I. V. Dedova, M. Tsubakihara, D. A. Berry and N. J. Nosworthy (2003). "Actin binding proteins: regulation of cytoskeletal microfilaments." Physiol Rev 83(2): 433-473.

Drewes, G., A. Ebneth, U. Preuss, E. M. Mandelkow and E. Mandelkow (1997). "MARK, a novel family of protein kinases that phosphorylate microtubule-associated proteins and trigger microtubule disruption." Cell 89(2): 297-308.

Drubin, D. G. and M. W. Kirschner (1986). "Tau protein function in living cells." J Cell Biol 103(6 Pt 2): 2739-2746.

Egelman, E. H. (1985). "The structure of F-actin." J Muscle Res Cell Motil 6(2): 129-151.

Egelman, E. H. (2003). "A tale of two polymers: new insights into helical filaments." Nat Rev Mol Cell Biol 4(8): 621-630.

Egelman, E. H., N. Francis and D. J. DeRosier (1982). "F-actin is a helix with a random variable twist." Nature 298(5870): 131-135.

Elie, A., E. Prezel, C. Guerin, E. Denarier, S. Ramirez-Rios, L. Serre, A. Andrieux, A. FourestLieuvin, L. Blanchoin and I. Arnal (2015). "Tau co-organizes dynamic microtubule and actin networks." Sci Rep 5: 9964.

Farias, G. A., J. P. Munoz, J. Garrido and R. B. Maccioni (2002). "Tubulin, actin, and tau protein interactions and the study of their macromolecular assemblies." Journal of Cellular Biochemistry 85(2): 315-324.

Fauquant, C., V. Redeker, I. Landrieu, J. M. Wieruszeski, D. Verdegem, O. Laprevote, G. Lippens, B. Gigant and M. Knossow (2011). "Systematic identification of tubulin-interacting fragments of the microtubule-associated protein Tau leads to 
a highly efficient promoter of microtubule assembly." J Biol Chem 286(38): 33358-33368.

Fischer, M., S. Kaech, D. Knutti and A. Matus (1998). "Rapid actin-based plasticity in dendritic spines." Neuron 20(5): 847-854.

Frandemiche, M. L., S. De Seranno, T. Rush, E. Borel, A. Elie, I. Arnal, F. Lante and A. Buisson (2014). "Activity-dependent tau protein translocation to excitatory synapse is disrupted by exposure to amyloid-beta oligomers." J Neurosci 34(17): 60846097.

Frieden, C. (1982). "The Mg2+-Induced Conformational Change in Rabbit Skeletal-Muscle GActin." Journal of Biological Chemistry 257(6): 2882-2886.

Fujii, T., A. H. Iwane, T. Yanagida and K. Namba (2010). "Direct visualization of secondary structures of F-actin by electron cryomicroscopy." Nature 467(7316): 724-U117.

Fulga, T. A., I. Elson-Schwab, V. Khurana, M. L. Steinhilb, T. L. Spires, B. T. Hyman and M. B. Feany (2007). "Abnormal bundling and accumulation of F-actin mediates tauinduced neuronal degeneration in vivo." Nature Cell Biology 9(2): 139-U117.

Fulga, T. A., I. Elson-Schwab, V. Khurana, M. L. Steinhilb, T. L. Spires, B. T. Hyman and M. B. Feany (2007). "Abnormal bundling and accumulation of F-actin mediates tauinduced neuronal degeneration in vivo." Nat Cell Biol 9(2): 139-148.

Galkin, V. E., A. Orlova, D. S. Kudryashov, A. Solodukhin, E. Reisler, G. F. Schroder and E. H. Egelman (2011). "Remodeling of actin filaments by ADF/cofilin proteins." Proceedings of the National Academy of Sciences of the United States of America 108(51): 20568-20572.

Galkin, V. E., A. Orlova, G. F. Schroder and E. H. Egelman (2010). "Structural polymorphism in F-actin." Nature Structural \& Molecular Biology 17(11): 1318-U1169.

Giannakopoulos, P., F. R. Herrmann, T. Bussiere, C. Bouras, E. Kovari, D. P. Perl, J. H. Morrison, G. Gold and P. R. Hof (2003). "Tangle and neuron numbers, but not amyloid load, predict cognitive status in Alzheimer's disease." Neurology 60(9): 1495-1500.

Giannetti, A. M., G. Lindwall, M. F. Chau, M. J. Radeke, S. C. Feinstein and L. A. Kohlstaedt (2000). "Fibers of tau fragments, but not full length tau, exhibit a cross betastructure: implications for the formation of paired helical filaments." Protein Sci 9(12): 2427-2435.

Gigant, B., I. Landrieu, C. Fauquant, P. Barbier, I. Huvent, J. M. Wieruszeski, M. Knossow and G. Lippens (2014). "Mechanism of Tau-Promoted Microtubule Assembly As Probed by NMR Spectroscopy." Journal of the American Chemical Society 136(36): 12615-12623.

Gillespie, J. R. and D. Shortle (1997). "Characterization of long-range structure in the denatured state of staphylococcal nuclease. I. Paramagnetic relaxation enhancement by nitroxide spin labels." J Mol Biol 268(1): 158-169.

Goddette, D. W. and C. Frieden (1985). "The binding of cytochalasin D to monomeric actin." Biochemical and Biophysical Research Communications 128(3): 1087-1092. 
Godoy, J. A., J. A. Rios, J. M. Zolezzi, N. Braidy and N. C. Inestrosa (2014). "Signaling pathway cross talk in Alzheimer's disease." Cell Commun Signal 12: 23.

GomezIsla, T., J. L. Price, D. W. McKeel, J. C. Morris, J. H. Growdon and B. T. Hyman (1996). "Profound loss of layer II entorhinal cortex neurons occurs in very mild Alzheimer's disease." Journal of Neuroscience 16(14): 4491-4500.

Gong, C. X., F. Liu, I. Grundke-Iqbal and K. Iqbal (2005). "Post-translational modifications of tau protein in Alzheimer's disease." J Neural Transm 112(6): 813-838.

Goode, B. L. and S. C. Feinstein (1994). "Identification of a novel microtubule binding and assembly domain in the developmentally regulated inter-repeat region of tau." J Cell Biol 124(5): 769-782.

Gorbatyuk, V. Y., N. J. Nosworthy, S. A. Robson, N. P. Bains, M. W. Maciejewski, C. G. Dos Remedios and G. F. King (2006). "Mapping the phosphoinositide-binding site on chick cofilin explains how PIP2 regulates the cofilin-actin interaction." Mol Cell 24(4): 511-522.

Griffith, L. M. and T. D. Pollard (1978). "Evidence for actin filament-microtubule interaction mediated by microtubule-associated proteins." J Cell Biol 78(3): 958-965.

Griffith, L. M. and T. D. Pollard (1982). "The interaction of actin filaments with microtubules and microtubule-associated proteins." J Biol Chem 257(15): 9143-9151.

Gronenborn, G. M. C. a. A. M. (1983). "Theory of the Time Dependent Transferred Nuclear Overhauser Effect: Applications to Structural Analysis of Ligand-Protein Complexes in Solution." Journal of Magnetic Resonance 53: 423-442.

Güntert, P. and L. Buchner (2015). "Combined automated NOE assignment and structure calculation with CYANA." Journal of Biomolecular NMR 62(4): 453-471.

Guo, K. K., J. Shillcock and R. Lipowsky (2010). "Treadmilling of actin filaments via Brownian dynamics simulations." Journal of Chemical Physics 133(15).

Gustke, N., B. Trinczek, J. Biernat, E. M. Mandelkow and E. Mandelkow (1994). "Domains of tau protein and interactions with microtubules." Biochemistry 33(32): 95119522.

Gustke, N., B. Trinczek, J. Biernat, E. M. Mandelkow and E. Mandelkow (1994). "Domains of Tau-Protein and Interactions with Microtubules." Biochemistry 33(32): 95119522.

Hanger, D. P., B. H. Anderton and W. Noble (2009). "Tau phosphorylation: the therapeutic challenge for neurodegenerative disease." Trends in Molecular Medicine 15(3): 112-119.

Hanger, D. P., K. Hughes, J. R. Woodgett, J. P. Brion and B. H. Anderton (1992). "Glycogen synthase kinase-3 induces Alzheimer's disease-like phosphorylation of tau: generation of paired helical filament epitopes and neuronal localisation of the kinase." Neurosci Lett 147(1): 58-62.

He, H. J., X. S. Wang, R. Pan, D. L. Wang, M. N. Liu and R. Q. He (2009). "The proline-rich domain of tau plays a role in interactions with actin." BMC Cell Biol 10: 81. 
He, H. J., X. S. Wang, R. Pan, D. L. Wang, M. N. Liu and R. Q. He (2009). "The proline-rich domain of tau plays a role in interactions with actin." Bmc Cell Biology 10.

Henriquez, J. P., D. Cross, C. Vial and R. B. Maccioni (1995). "Subpopulations of Tau Interact with Microtubules and Actin-Filaments in Various Cell-Types." Cell Biochemistry and Function 13(4): 239-250.

Herman, I. M. (1993). "Actin isoforms." Curr Opin Cell Biol 5(1): 48-55.

Hertzog, M., C. van Heijenoort, D. Didry, M. Gaudier, J. Coutant, B. Gigant, G. Didelot, T. Preat, M. Knossow, E. Guittet and M. F. Carlier (2004). "The betathymosin/WH2 domain: Structural basis for the switch from inhibition to promotion of actin assembly." Cell 117(5): 611-623.

Hertzog, M., E. G. Yarmola, D. Didry, M. R. Bubb and M. F. Carlier (2002). "Control of actin dynamics by proteins made of beta-thymosin repeats - The actobindin family." Journal of Biological Chemistry 277(17): 14786-14792.

Hetenyi, A., Z. Hegedus, R. Fajka-Boja, E. Monostori, K. E. Kover and T. A. Martinek (2016). "Target-specific NMR detection of protein-ligand interactions with antibodyrelayed 15N-group selective STD." J Biomol NMR 66(4): 227-232.

Hirano, A. (1994). "Hirano bodies and related neuronal inclusions." Neuropathol Appl Neurobiol 20(1): 3-11.

Holmes, K. C., D. Popp, W. Gebhard and W. Kabsch (1990). "Atomic model of the actin filament." Nature 347(6288): 44-49.

Holzinger, A. and K. Blaas (2016). "Actin-Dynamics in Plant Cells: The Function of ActinPerturbing Substances: Jasplakinolide, Chondramides, Phalloidin, Cytochalasins, and Latrunculins." Methods Mol Biol 1365: 243-261.

Hoover, B. R., M. N. Reed, J. J. Su, R. D. Penrod, L. A. Kotilinek, M. K. Grant, R. Pitstick, G. A. Carlson, L. M. Lanier, L. L. Yuan, K. H. Ashe and D. Z. Liao (2010). "Tau Mislocalization to Dendritic Spines Mediates Synaptic Dysfunction Independently of Neurodegeneration." Neuron 68(6): 1067-1081.

Hotulainen, P. and C. C. Hoogenraad (2010). "Actin in dendritic spines: connecting dynamics to function." J Cell Biol 189(4): 619-629.

Huvent, I., A. Kamah, F. X. Cantrelle, N. Barois, C. Slomianny, C. Smet-Nocca, I. Landrieu and G. Lippens (2014). "A functional fragment of Tau forms fibers without the need for an intermolecular cysteine bridge." Biochem Biophys Res Commun 445(2): 299-303.

Illenberger, S., Q. Y. Zheng-Fischhofer, U. Preuss, K. Stamer, K. Baumann, B. Trinczek, J. Biernat, R. Godemann, E. M. Mandelkow and E. Mandelkow (1998). "The endogenous and cell cycle-dependent phosphorylation of tau protein in living cells: Implications for Alzheimer's disease." Molecular Biology of the Cell 9(6): 1495-1512.

Iqbal, K., A. D. C. Alonso, S. Chen, M. O. Chohan, E. El-Akkad, C. X. Gong, S. Khatoon, B. Li, F. Liu, A. Rahman, H. Tanimukai and I. Grundke-Iqbal (2005). "Tau pathology in Alzheimer disease and other tauopathies." Biochimica Et Biophysica ActaMolecular Basis of Disease 1739(2-3): 198-210. 
Jeganathan, S., A. Hascher, S. Chinnathambi, J. Biernat, E. M. Mandelkow and E. Mandelkow (2008). "Proline-directed Pseudo-phosphorylation at AT8 and PHF1 Epitopes Induces a Compaction of the Paperclip Folding of Tau and Generates a Pathological (MC-1) Conformation." Journal of Biological Chemistry 283(46): 32066-32076.

Jeganathan, S., M. von Bergen, H. Brutlach, H. J. Steinhoff and E. Mandelkow (2006). "Global hairpin folding of tau in solution." Biochemistry 45(7): 2283-2293.

Kabsch, W., H. G. Mannherz, D. Suck, E. F. Pai and K. C. Holmes (1990). "Atomic structure of the actin:DNase I complex." Nature 347(6288): 37-44.

Kadavath, H., R. V. Hofele, J. Biernat, S. Kumar, K. Tepper, H. Urlaub, E. Mandelkow and M. Zweckstetter (2015). "Tau stabilizes microtubules by binding at the interface between tubulin heterodimers." Proc Natl Acad Sci U S A 112(24): 7501-7506.

Kadavath, H., M. Jaremko, L. Jaremko, J. Biernat, E. Mandelkow and M. Zweckstetter (2015). "Folding of the Tau Protein on Microtubules." Angewandte ChemieInternational Edition 54(35): 10347-10351.

Kar, S., J. Fan, M. J. Smith, M. Goedert and L. A. Amos (2003). "Repeat motifs of tau bind to the insides of microtubules in the absence of taxol." Embo Journal 22(1): 70-77.

Kasai, H., M. Matsuzaki, J. Noguchi, N. Yasumatsu and H. Nakahara (2003). "Structurestability-function relationships of dendritic spines." Trends in Neurosciences 26(7): 360-368.

Kashman, Y., A. Groweiss and J. Shmueli (1980). "Latrunculin, a New 2-Thiazolidinone Macrolide from the Marine Sponge Latrunculia-Magnifica." Tetrahedron Letters 21(37): 3629-3632.

Kinosian, H. J., L. A. Selden, J. E. Estes and L. C. Gershman (1993). "Nucleotide binding to actin. Cation dependence of nucleotide dissociation and exchange rates." J Biol Chem 268(12): 8683-8691.

Klein, C., E. M. Kramer, A. M. Cardine, B. Schraven, R. Brandt and J. Trotter (2002). "Process outgrowth of oligodendrocytes is promoted by interaction of Fyn kinase with the cytoskeletal protein Tau." Journal of Neuroscience 22(3): 698-707.

Klenchin, V. A., J. S. Allingham, R. King, J. Tanaka, G. Marriott and I. Rayment (2003). "Trisoxazole macrolide toxins mimic the binding of actin-capping proteins to actin." Nat Struct Biol 10(12): 1058-1063.

Knops, J., K. S. Kosik, G. Lee, J. D. Pardee, L. Cohen-Gould and L. McConlogue (1991). "Overexpression of tau in a nonneuronal cell induces long cellular processes." J Cell Biol 114(4): 725-733.

Kolli, R. P., R. M. Wojes, S. Zaucha and D. N. Seidman (2008). "A subnanoscale study of the nucleation, growth, and coarsening kinetics of $\mathrm{Cu}$-rich precipitates in a multicomponent Fe-Cu based steel." International Journal of Materials Research 99(5): 513-527.

Koradi, R., M. Billeter and K. Wuthrich (1996). "MOLMOL: a program for display and analysis of macromolecular structures." J Mol Graph 14(1): 51-55, 29-32. 
Kunze, B., R. Jansen, F. Sasse, G. Hofle and H. Reichenbach (1995). "Chondramides A approximately D, new antifungal and cytostatic depsipeptides from Chondromyces crocatus (myxobacteria). Production, physico-chemical and biological properties." J Antibiot (Tokyo) 48(11): 1262-1266.

Kunze, G., P. Barre, H. A. Scheidt, L. Thomas, D. Eliezer and D. Huster (2012). "Binding of the three-repeat domain of tau to phospholipid membranes induces an aggregatedlike state of the protein." Biochim Biophys Acta 1818(9): 2302-2313.

Lazarides, E. and K. Weber (1974). "Actin antibody: the specific visualization of actin filaments in non-muscle cells." Proc Natl Acad Sci U S A 71(6): 2268-2272.

Lee, G. (1993). "Non-motor microtubule-associated proteins." Curr Opin Cell Biol 5(1): 88-94.

Lee, V. M. Y., M. Goedert and J. Q. Trojanowski (2001). "Neurodegenerative tauopathies." Annual Review of Neuroscience 24: 1121-1159.

Lee, W. M. and R. M. Galbraith (1992). "The extracellular actin-scavenger system and actin toxicity." N Engl J Med 326(20): 1335-1341.

Lodish H, B. A., Zipursky SL, et al. (2000). Molecular Cell Biology. W. H. Freeman. New York.

Loomis, P. A., T. H. Howard, R. P. Castleberry and L. I. Binder (1990). "Identification of Nuclear Tau-Isoforms in Human Neuroblastoma-Cells." Proceedings of the National Academy of Sciences of the United States of America 87(21): 84228426.

Lorenz, M., D. Popp and K. C. Holmes (1993). "Refinement of the F-Actin Model against X-Ray Fiber Diffraction Data by the Use of a Directed Mutation Algorithm." Journal of Molecular Biology 234(3): 826-836.

Lu, P. J., G. Wulf, X. Z. Zhou, P. Davies and K. P. Lu (1999). "The prolyl isomerase Pin1 restores the function of Alzheimer-associated phosphorylated tau protein." Nature 399(6738): 784-788.

Lucas, J. J., F. Hernandez, P. Gomez-Ramos, M. A. Moran, R. Hen and J. Avila (2001). "Decreased nuclear beta-catenin, tau hyperphosphorylation and neurodegeneration in GSK-3beta conditional transgenic mice." EMBO J 20(1-2): 27-39.

Macchi, E., T. R. Rudd, R. Raman, R. Sasisekharan, E. A. Yates, A. Naggi, M. Guerrini and S. Elli (2016). "Nuclear Magnetic Resonance and Molecular Dynamics Simulation of the Interaction between Recognition Protein H7 of the Novel Influenza Virus H7N9 and Glycan Cell Surface Receptors." Biochemistry 55(48): 6605-6616.

Makioka, K., T. Yamazaki, M. Takatama, M. Ikeda and K. Okamoto (2014). "Immunolocalization of Smurf1 in Hirano bodies." J Neurol Sci 336(1-2): 24-28.

Makrides, V., M. R. Massie, S. C. Feinstein and J. Lew (2004). "Evidence for two distinct binding sites for tau on microtubules." Proceedings of the National Academy of Sciences of the United States of America 101(17): 6746-6751.

Mandelkow, E. and E. M. Mandelkow (1995). "Microtubules and microtubule-associated proteins." Curr Opin Cell Biol 7(1): 72-81. 
Mannherz, H. G., R. S. Goody, M. Konrad and E. Nowak (1980). "The Interaction of Bovine Pancreatic Deoxyribonuclease-I and Skeletal-Muscle Actin." European Journal of Biochemistry 104(2): 367-379.

Mannherz, H. G., J. B. Leigh, R. Leberman and H. Pfrang (1975). "Specific 1-1 G-Actin Dnaase-I Complex Formed by Action of Dnaase-I on F-Actin." Febs Letters 60(1): 34-38.

Margittai, M. and R. Langen (2004). "Template-assisted filament growth by parallel stacking of tau." Proc Natl Acad Sci U S A 101(28): 10278-10283.

Margittai, M. and R. Langen (2006). "Side chain-dependent stacking modulates tau filament structure." J Biol Chem 281(49): 37820-37827.

Martin, L., X. Latypova and F. Terro (2011). "Post-translational modifications of tau protein: Implications for Alzheimer's disease." Neurochemistry International 58(4): 458471.

Martin, L., X. Latypova, C. M. Wilson, A. Magnaudeix, M. L. Perrin, C. Yardin and F. Terro (2013). "Tau protein kinases: Involvement in Alzheimer's disease." Ageing Research Reviews 12(1): 289-309.

Matenia, D. and E. M. Mandelkow (2009). "The tau of MARK: a polarized view of the cytoskeleton." Trends in Biochemical Sciences 34(7): 332-342.

Matthews, J. B., J. A. Smith and B. J. Hrnjez (1997). "Effects of F-actin stabilization or disassembly on epithelial Cl- secretion and Na-K-2Cl cotransport." Am J Physiol 272(1 Pt 1): C254-262.

Mattila, P. K., O. Quintero-Monzon, J. Kugler, J. B. Moseley, S. C. Almo, P. Lappalainen and B. L. Goode (2004). "A high-affinity interaction with ADP-actin monomers underlies the mechanism and in vivo function of Srv2/cyclase-associated protein." Molecular Biology of the Cell 15(11): 5158-5171.

Mayer, M. and B. Meyer (1999). "Characterization of Ligand Binding by Saturation Transfer Difference NMR Spectroscopy." Angewandte Chemie International Edition 38(12): 1784-1788.

McConkey BJ, S. V., Edelman M. (2002). "The performance of current methods in ligandprotein docking." Current Science 83(07): 845-855.

Mclaughlin, P. J., J. T. Gooch, H. G. Mannherz and A. G. Weeds (1993). "Structure of Gelsolin Segment-1-Actin Complex and the Mechanism of Filament Severing." Nature 364(6439): 685-692.

Melo, A. M., J. Coraor, G. Alpha-Cobb, S. Elbaum-Garfinkle, A. Nath and E. Rhoades (2016). "A functional role for intrinsic disorder in the tau-tubulin complex." Proc Natl Acad Sci U S A 113(50): 14336-14341.

Merlini, M., D. Wanner and R. M. Nitsch (2016). "Tau pathology-dependent remodelling of cerebral arteries precedes Alzheimer's disease-related microvascular cerebral amyloid angiopathy." Acta Neuropathol 131(5): 737-752.

Min, S. W., S. H. Cho, Y. G. Zhou, S. Schroeder, V. Haroutunian, W. W. Seeley, E. J. Huang, Y. Shen, E. Masliah, C. Mukherjee, D. Meyers, P. A. Cole, M. Ott and L. Gan 
(2010). "Acetylation of Tau Inhibits Its Degradation and Contributes to Tauopathy (vol 67, pg 953, 2010)." Neuron 68(4): 801-801.

Minamide, L. S., A. M. Striegl, J. A. Boyle, P. J. Meberg and J. R. Bamburg (2000). "Neurodegenerative stimuli induce persistent ADF/cofilin-actin rods that disrupt distal neurite function." Nat Cell Biol 2(9): 628-636.

Moraczewska, J., B. Wawro, K. Seguro and H. Strzelecka-Golaszewska (1999). "Divalent cation-, nucleotide-, and polymerization-dependent changes in the conformation of subdomain 2 of actin." Biophysical Journal 77(1): 373-385.

Moradi, M., R. Sivadasan, L. Saal, P. Luningschror, B. Dombert, R. J. Rathod, D. C. Dieterich, R. Blum and M. Sendtner (2017). "Differential roles of alpha-, beta-, and gamma-actin in axon growth and collateral branch formation in motoneurons." J Cell Biol 216(3): 793-814.

Moraga, D. M., P. Nunez, J. Garrido and R. B. Maccioni (1993). "A tau fragment containing a repetitive sequence induces bundling of actin filaments." J Neurochem 61(3): 979-986.

Morishima, M. and Y. Ihara (1994). "Posttranslational modifications of tau in paired helical filaments." Dementia 5(5): 282-288.

Morris, M., S. Maeda, K. Vossel and L. Mucke (2011). "The Many Faces of Tau." Neuron 70(3): 410-426.

Muhlrad, A., D. Pavlov, Y. M. Peyser and E. Reisler (2006). "Inorganic phosphate regulates the binding of cofilin to actin filaments." Febs Journal 273(7): 1488-1496.

Mukrasch, M. D., S. Bibow, J. Korukottu, S. Jeganathan, J. Biernat, C. Griesinger, E. Mandelkow and M. Zweckstetter (2009). "Structural Polymorphism of 441Residue Tau at Single Residue Resolution." Plos Biology 7(2): 399-414.

Mylonas, E., A. Hascher, P. Bernado, M. Blackledge, E. Mandelkow and D. I. Svergun (2008). "Domain conformation of tau protein studied by solution small-angle X-ray scattering." Biochemistry 47(39): 10345-10353.

Nam, W. and B. I. Epureanu (2017). "Dynamic model for kinesin-mediated long-range transport and its local traffic jam caused by tau proteins." Phys Rev E 95(1-1): 012405.

Narayanan, R. L., U. H. N. Durr, S. Bibow, J. Biernat, E. Mandelkow and M. Zweckstetter (2010). "Automatic Assignment of the Intrinsically Disordered Protein Tau with 441-Residues." Journal of the American Chemical Society 132(34): 1190611907.

Ni, F. (1994). "Recent developments in transferred NOE methods." Progress in Nuclear Magnetic Resonance Spectroscopy 26, Part 6: 517-606.

Ni, F. and H. A. Scheraga (1994). "Use of the Transferred Nuclear Overhauser Effect To Determine the Conformations of Ligands Bound to Proteins." Accounts of Chemical Research 27(9): 257-264.

Nishida, E. (1985). "Opposite Effects of Cofilin and Profilin from Porcine Brain on Rate of Exchange of Actin-Bound Adenosine 5'-Triphosphate." Biochemistry 24(5): 1160-1164. 
Nuzillard, J. M. and G. Massiot (1991). "Two-dimensional extensions of 1D HOHAHA experiments." Journal of Magnetic Resonance (1969) 91(2): 380-385.

O'Connell, M. R., R. Gamsjaeger and J. P. Mackay (2009). "The structural analysis of proteinprotein interactions by NMR spectroscopy." Proteomics 9(23): 5224-5232.

Oda, T., M. Iwasa, T. Aihara, Y. Maeda and A. Narita (2009). "The nature of the globular-to fibrous-actin transition." Nature 457(7228): 441-445.

Ojala, P. J., V. O. Paavilainen, M. K. Vartiainen, R. Tuma, A. G. Weeds and P. Lappalainen (2002). "The two ADF-H domains of twinfilin play functionally distinct roles in interactions with actin monomers." Molecular Biology of the Cell 13(11): 38113821.

Ozer, R. S. and S. Halpain (2000). "Phosphorylation-dependent localization of microtubuleassociated protein MAP2c to the actin cytoskeleton." Mol Biol Cell 11(10): 3573-3587.

Pavlov, D., A. Muhlrad, J. Cooper, M. Wear and E. Reisler (2006). "Severing of F-actin by yeast cofilin is pH-independent." Cell Motility and the Cytoskeleton 63(9): 533-542.

Pedrotti, B., R. Colombo and K. Islam (1994). "Interactions of microtubule-associated protein MAP2 with unpolymerized and polymerized tubulin and actin using a 96-well microtiter plate solid-phase immunoassay." Biochemistry 33(29): 8798-8806.

Penzes, P. and J. E. VanLeeuwen (2011). "Impaired regulation of synaptic actin cytoskeleton in Alzheimer’s disease." Brain Res Rev 67(1-2): 184-192.

Peterson, J. R. and T. J. Mitchison (2002). "Small molecules, big impact: a history of chemical inhibitors and the cytoskeleton." Chem Biol 9(12): 1275-1285.

Pollard, T. D., L. Blanchoin and R. D. Mullins (2000). "Molecular mechanisms controlling actin filament dynamics in nonmuscle cells." Annual Review of Biophysics and Biomolecular Structure 29: 545-576.

Pollard, T. D. and G. G. Borisy (2003). "Cellular motility driven by assembly and disassembly of actin filaments." Cell 112(4): 453-465.

Pollard, T. D. and J. A. Cooper (1984). "Quantitative analysis of the effect of Acanthamoeba profilin on actin filament nucleation and elongation." Biochemistry 23(26): 6631-6641.

Pollard, T. D., E. Shelton, R. R. Weihing and E. D. Korn (1970). "Ultrastructural Characterization of F-Actin Isolated from Acanthamoeba-Castellanii and Identification of Cytoplasmic Filaments as F-Actin by Reaction with Rabbit Heavy Meromyosin." Journal of Molecular Biology 50(1): 91-\&.

Pope, B. J., K. M. Zierler-Gould, R. Kuhne, A. G. Weeds and L. J. Ball (2004). "Solution structure of human cofilin: actin binding, $\mathrm{pH}$ sensitivity, and relationship to actin-depolymerizing factor." J Biol Chem 279(6): 4840-4848.

Post, C. B. (2003). "Exchange-transferred NOE spectroscopy and bound ligand structure determination." Current Opinion in Structural Biology 13(5): 581-588.

Prince, M., A. Wimo, M. Guerchet, G.-C. Ali, Y.-T. Wu and M. Prina (2015). "World Alzheimer Report 2015. The Global Impact of Dementia: An analysis of prevalence, 
incidence, cost and trends." Alzheimer’s Disease International (ADI), London.: $1-82$.

Pring, M., A. Weber and M. R. Bubb (1992). "Profilin-actin complexes directly elongate actin filaments at the barbed end." Biochemistry 31(6): 1827-1836.

Rajagopal, P., E. B. Waygood, J. Reizer, M. H. Saier, Jr. and R. E. Klevit (1997). "Demonstration of protein-protein interaction specificity by NMR chemical shift mapping." Protein Sci 6(12): 2624-2627.

Ramirez-Bermudez, J. (2012). "Alzheimer's Disease: Critical Notes on the History of a Medical Concept." Archives of Medical Research 43(8): 595-599.

Rodriguez, O. C., A. W. Schaefer, C. A. Mandato, P. Forscher, W. M. Bement and C. M. Waterman-Storer (2003). "Conserved microtubule-actin interactions in cell movement and morphogenesis." Nat Cell Biol 5(7): 599-609.

Rould, M. A., Q. Wan, P. B. Joel, S. Lowey and K. M. Trybus (2006). "Crystal structures of expressed non-polymerizable monomeric actin in the ADP and ATP states." J Biol Chem 281(42): 31909-31919.

Santarella, R. A., G. Skiniotis, K. N. Goldie, P. Tittmann, H. Gross, E. M. Mandelkow, E. Mandelkow and A. Hoenger (2004). "Surface-decoration of microtubules by human Tau." Journal of Molecular Biology 339(3): 539-553.

Sasse, F., B. Kunze, T. M. Gronewold and H. Reichenbach (1998). "The chondramides: cytostatic agents from myxobacteria acting on the actin cytoskeleton." J Natl Cancer Inst 90(20): 1559-1563.

Sasse, F., B. Kunze, T. M. A. Gronewold and H. Reichenbach (1998). "The chondramides: Cytostatic agents from myxobacteria acting on the actin cytoskeleton." Journal of the National Cancer Institute 90(20): 1559-1563.

Sattilaro, R. F. (1986). "Interaction of microtubule-associated protein 2 with actin filaments." Biochemistry 25(8): 2003-2009.

Sawitzky, H., S. Liebe, J. Willingale-Theune and D. Menzel (1999). "The anti-proliferative agent jasplakinolide rearranges the actin cytoskeleton of plant cells." European Journal of Cell Biology 78(6): 424-433.

Schuler, H. (2001). "ATPase activity and conformational changes in the regulation of actin." Biochimica Et Biophysica Acta-Protein Structure and Molecular Enzymology 1549(2): 137-147.

Schutt, C. E., C. Kreatsoulas, R. Page and U. Lindberg (1997). "Plugging into actin's architectonic socket." Nature Structural Biology 4(3): 169-172.

Schutt, C. E., M. D. Rozycki, J. C. Myslik and U. Lindberg (1995). "A Discourse on Modeling F-Actin." Journal of Structural Biology 115(2): 186-198.

Schwalbe, M., J. Biernat, S. Bibow, V. Ozenne, M. R. Jensen, H. Kadavath, M. Blackledge, E. Mandelkow and M. Zweckstetter (2013). "Phosphorylation of Human Tau Protein by Microtubule Affinity-Regulating Kinase 2." Biochemistry 52(50): 9068-9079. 
Selden, S. C. and T. D. Pollard (1983). "Phosphorylation of microtubule-associated proteins regulates their interaction with actin filaments." J Biol Chem 258(11): 70647071.

Sergeant, N., A. Delacourte and L. Buee (2005). "Tau protein as a differential biomarker of tauopathies." Biochimica Et Biophysica Acta-Molecular Basis of Disease 1739(2-3): 179-197.

Shoji, K., K. Ohashi, K. Sampei, M. Oikawa and K. Mizuno (2012). "Cytochalasin D acts as an inhibitor of the actin-cofilin interaction." Biochem Biophys Res Commun 424(1): 52-57.

Silacci, P., L. Mazzolai, C. Gauci, N. Stergiopulos, H. L. Yin and D. Hayoz (2004). "Gelsolin superfamily proteins: key regulators of cellular functions." Cell Mol Life Sci 61(19-20): 2614-2623.

Sokolov, M. A., S. Spooner, G. R. Odette, B. D. Wirth and G. E. Lucas (1999). "Sans study of high-copper RPV welds in irradiated and annealed conditions." Effects of Radiation on Materials: 18th International Symposium 1325: 333-345.

Sottejeau, Y., A. Bretteville, F. X. Cantrelle, N. Malmanche, F. Demiaute, T. Mendes, C. Delay, H. A. Dos Alves, A. Flaig, P. Davies, P. Dourlen, B. Dermaut, J. Laporte, P. Amouyel, G. Lippens, J. Chapuis, I. Landrieu and J. C. Lambert (2015). "Tau phosphorylation regulates the interaction between BIN1's SH3 domain and Tau's proline-rich domain." Acta Neuropathologica Communications 3.

Spears, W., M. Furgerson, J. M. Sweetnam, P. Evans, M. Gearing, M. Fechheimer and R. Furukawa (2014). "Hirano bodies differentially modulate cell death induced by tau and the amyloid precursor protein intracellular domain." BMC Neurosci 15: 74.

Spector, I., N. R. Shochet, Y. Kashman and A. Groweiss (1983). "Latrunculins - Novel Marine Toxins That Disrupt Microfilament Organization in Cultured-Cells." Science 219(4584): 493-495.

Spittaels, K., C. Van den Haute, J. Van Dorpe, H. Geerts, M. Mercken, K. Bruynseels, R. Lasrado, K. Vandezande, I. Laenen, T. Boon, J. Van Lint, J. Vandenheede, D. Moechars, R. Loos and F. Van Leuven (2000). "Glycogen synthase kinase-3beta phosphorylates protein tau and rescues the axonopathy in the central nervous system of human four-repeat tau transgenic mice." J Biol Chem 275(52): 4134041349

Star, E. N., D. J. Kwiatkowski and V. N. Murthy (2002). "Rapid turnover of actin in dendritic spines and its regulation by activity." Nature Neuroscience 5(3): 239-246.

Stern, J. L., D. V. Lessard, G. J. Hoeprich, G. A. Morfini and C. L. Berger (2017). "Phosphoregulation of tau modulates inhibition of kinesin-1 motility." Mol Biol Cell.

Stricker, J., T. Falzone and M. L. Gardel (2010). "Mechanics of the F-actin cytoskeleton." Journal of Biomechanics 43(1): 9-14.

Suarez, C., J. Roland, R. Boujemaa-Paterski, H. Kang, B. R. McCullough, A. C. Reymann, C. Guerin, J. L. Martiel, E. M. De La Cruz and L. Blanchoin (2011). "Cofilin Tunes the Nucleotide State of Actin Filaments and Severs at Bare and Decorated Segment Boundaries." Current Biology 21(10): 862-868. 
Tanaka, J., Y. Yan, J. Choi, J. Bai, V. A. Klenchin, I. Rayment and G. Marriott (2003). "Biomolecular mimicry in the actin cytoskeleton: mechanisms underlying the cytotoxicity of kabiramide C and related macrolides." Proc Natl Acad Sci U S A 100(24): 13851-13856.

Tang, B., Y. Huang, H. Yang, P. Tang and H. Li (2016). "Molecular mechanism of the binding of 3,4,5-tri-O-caffeoylquinic acid to human serum albumin: Saturation transfer difference NMR, multi-spectroscopy, and docking studies." J Photochem Photobiol B 165: 24-33.

Tashiro, K., M. Hasegawa, Y. Ihara and T. Iwatsubo (1997). "Somatodendritic localization of phosphorylated tau in neonatal and adult rat cerebral cortex." Neuroreport 8(12): 2797-2801.

Torres-Cruz, F. M., F. Rodriguez-Cruz, J. Escobar-Herrera, N. Barragan-Andrade, G. BasurtoIslas, D. Ripova, J. Avila and F. Garcia-Sierra (2016). "Expression of Tau Produces Aberrant Plasma Membrane Blebbing in Glial Cells Through RhoAROCK-Dependent F-Actin Remodeling." Journal of Alzheimers Disease 52(2): 463-482.

Torres-Cruz, F. M., F. Rodriguez-Cruz, J. Escobar-Herrera, N. Barragan-Andrade, G. BasurtoIslas, D. Ripova, J. Avila and F. Garcia-Sierra (2016). "Expression of Tau Produces Aberrant Plasma Membrane Blebbing in Glial Cells Through RhoAROCK-Dependent F-Actin Remodeling." J Alzheimers Dis 52(2): 463-482.

Tracy, T. E., P. D. Sohn, S. S. Minami, C. Wang, S. W. Min, Y. Li, Y. Zhou, D. Le, I. Lo, R. Ponnusamy, X. Cong, B. Schilling, L. M. Ellerby, R. L. Huganir and L. Gan (2016). "Acetylated Tau Obstructs KIBRA-Mediated Signaling in Synaptic Plasticity and Promotes Tauopathy-Related Memory Loss." Neuron 90(2): 245260.

Utton, M. A., A. Vandecandelaere, U. Wagner, C. H. Reynolds, G. M. Gibb, C. C. J. Miller, P. M. Bayley and B. H. Anderton (1997). "Phosphorylation of tau by glycogen synthase kinase 3 beta affects the ability of tau to promote microtubule selfassembly." Biochemical Journal 323: 741-747.

Uversky, V. N. (2015). "Intrinsically disordered proteins and their (disordered) proteomes in neurodegenerative disorders." Front Aging Neurosci 7: 18.

van Zundert, G. C. P., J. P. G. L. M. Rodrigues, M. Trellet, C. Schmitz, P. L. Kastritis, E. Karaca, A. S. J. Melquiond, M. van Dijk, S. J. de Vries and A. M. J. J. Bonvin (2016). "The HADDOCK2.2 Web Server: User-Friendly Integrative Modeling of Biomolecular Complexes." Journal of Molecular Biology 428(4): 720-725.

Vandekerckhove, J. and K. Weber (1978). "At least six different actins are expressed in a higher mammal: an analysis based on the amino acid sequence of the amino-terminal tryptic peptide." J Mol Biol 126(4): 783-802.

Viegas, A., J. Manso, F. L. Nobrega and E. J. Cabrita (2011). "Saturation-Transfer Difference (STD) NMR: A Simple and Fast Method for Ligand Screening and Characterization of Protein Binding." Journal of Chemical Education 88(7): 990994. 
Vinson, V. K., E. M. De la Cruz, H. N. Higgs and T. D. Pollard (1998). "Interactions of Acanthamoeba profilin with actin and nucleotides bound to actin." Biochemistry 37(31): 10871-10880.

von Bergen, M., S. Barghorn, L. Li, A. Marx, J. Biernat, E. M. Mandelkow and E. Mandelkow (2001). "Mutations of tau protein in frontotemporal dementia promote aggregation of paired helical filaments by enhancing local beta-structure." J Biol Chem 276(51): 48165-48174.

von Bergen, M., S. Barghorn, S. A. Muller, M. Pickhardt, J. Biernat, E. M. Mandelkow, P. Davies, U. Aebi and E. Mandelkow (2006). "The core of tau-paired helical filaments studied by scanning transmission electron microscopy and limited proteolysis." Biochemistry 45(20): 6446-6457.

von Bergen, M., P. Friedhoff, J. Biernat, J. Heberle, E. M. Mandelkow and E. Mandelkow (2000). "Assembly of tau protein into Alzheimer paired helical filaments depends on a local sequence motif (306-VQIVYK-311) forming beta structure." Molecular Biology of the Cell 11: 363a-363a.

von Bergen, M., P. Friedhoff, J. Biernat, J. Heberle, E. M. Mandelkow and E. Mandelkow (2000). "Assembly of tau protein into Alzheimer paired helical filaments depends on a local sequence motif ((306)VQIVYK(311)) forming beta structure." Proc Natl Acad Sci U S A 97(10): 5129-5134.

von der Ecken, J., M. Muller, W. Lehman, D. J. Manstein, P. A. Penczek and S. Raunser (2015). "Structure of the F-actin-tropomyosin complex." Nature 519(7541): 114-117.

Wang, H., R. C. Robinson and L. D. Burtnick (2010). "The structure of native G-actin." Cytoskeleton (Hoboken) 67(7): 456-465.

Wang, J. Z., I. Grundke-Iqbal and K. Iqbal (1996). "Glycosylation of microtubule-associated protein tau: an abnormal posttranslational modification in Alzheimer's disease." Nat Med 2(8): 871-875.

Weingarten, M. D., A. H. Lockwood, S. Y. Hwo and M. W. Kirschner (1975). "A protein factor essential for microtubule assembly." Proc Natl Acad Sci U S A 72(5): 18581862.

Weingarten, M. D., A. H. Lockwood, S. Y. Hwo and M. W. Kirschner (1975). "A protein factor essential for microtubule assembly." Proceedings of the National Academy of Sciences 72(5): 1858-1862.

Whiteman, I. T., O. L. Gervasio, K. M. Cullen, G. J. Guillemin, E. V. Jeong, P. K. Witting, S. T. Antao, L. S. Minamide, J. R. Bamburg and C. Goldsbury (2009). "Activated actin-depolymerizing factor/cofilin sequesters phosphorylated microtubuleassociated protein during the assembly of alzheimer-like neuritic cytoskeletal striations." J Neurosci 29(41): 12994-13005.

Williams, D. R. (2006). "Tauopathies: classification and clinical update on neurodegenerative diseases associated with microtubule-associated protein tau." Intern Med J 36(10): 652-660.

Williamson, M. P. (2009). Chapter 3 Applications of the NOE in Molecular Biology. Annual Reports on NMR Spectroscopy, Academic Press. 65: 77-109. 
Witman, G. B., D. W. Cleveland, M. D. Weingarten and M. W. Kirschner (1976). "Tubulin requires tau for growth onto microtubule initiating sites." Proc Natl Acad Sci U S A 73(11): 4070-4074.

Wüthrich, K. (1986). NMR of Proteins and Nucleic Acids, Wiley.

Yamauchi, P. S. and D. L. Purich (1993). "Microtubule-associated protein interactions with actin filaments: evidence for differential behavior of neuronal MAP-2 and tau in the presence of phosphatidyl-inositol." Biochem Biophys Res Commun 190(3): 710715.

Yarmola, E. G., T. Somasundaram, T. A. Boring, I. Spector and M. R. Bubb (2000). "Actinlatrunculin A structure and function - Differential modulation of actin-binding protein function by latrunculin A." Journal of Biological Chemistry 275(36): 28120-28127.

Yasunaga, T. and T. Wakabayashi (2001). "Relocation of Cys374 of actin induced by labeling with fluorescent dyes." J Biochem 129(2): 201-204.

Yonezawa, N., E. Nishida, S. Koyasu, S. Maekawa, Y. Ohta, I. Yahara and H. Sakai (1987). "Distribution among tissues and intracellular localization of cofilin, a $21 \mathrm{kDa}$ actin-binding protein." Cell Struct Funct 12(5): 443-452.

Yoshida, H. and M. Goedert (2012). "Phosphorylation of microtubule-associated protein tau by AMPK-related kinases." Journal of Neurochemistry 120(1): 165-176.

Yu, J. Z. and M. M. Rasenick (2006). "Tau associates with actin in differentiating PC12 cells." Faseb Journal 20(9): 1452-1461.

Yu, W. D., J. Polepalli, D. Wagh, J. Rajadas, R. Malenka and B. W. Lu (2012). "A critical role for the PAR-1/MARK-tau axis in mediating the toxic effects of A on synapses and dendritic spines." Human Molecular Genetics 21(6): 1384-1390.

Yuzwa, S. A., X. Shan, M. S. Macauley, T. Clark, Y. Skorobogatko, K. Vosseller and D. J. Vocadlo (2012). "Increasing O-GlcNAc slows neurodegeneration and stabilizes tau against aggregation." Nat Chem Biol 8(4): 393-399.

Zarbock, J., H. Oschkinat, E. Hannappel, H. Kalbacher, W. Voelter and T. A. Holak (1990). "Solution Conformation of Thymosin-Beta-4 - a Nuclear-Magnetic-Resonance and Simulated Annealing Study." Biochemistry 29(34): 7814-7821.

Zempel, H., J. Luedtke, Y. Kumar, J. Biernat, H. Dawson, E. Mandelkow and E.-M. Mandelkow (2013). "Amyloid- $\beta$ oligomers induce synaptic damage via Tau-dependent microtubule severing by TTLL6 and spastin." The EMBO Journal 32(22): 29202937.

Zempel, H., E. Thies, E. Mandelkow and E. M. Mandelkow (2010). "A beta Oligomers Cause Localized Ca2+ Elevation, Missorting of Endogenous Tau into Dendrites, Tau Phosphorylation, and Destruction of Microtubules and Spines." Journal of Neuroscience 30(36): 11938-11950.

Zhang, B., A. Maiti, S. Shively, F. Lakhani, G. McDonald-Jones, J. Bruce, E. B. Lee, S. X. Xie, S. Joyce, C. Li, P. M. Toleikis, V. M. Lee and J. Q. Trojanowski (2005). "Microtubule-binding drugs offset tau sequestration by stabilizing microtubules 
and reversing fast axonal transport deficits in a tauopathy model." Proc Natl Acad Sci U S A 102(1): 227-231.

Zmuda, J. F. and R. J. Rivas (2000). "Actin disruption alters the localization of tau in the growth cones of cerebellar granule neurons." Journal of Cell Science 113(15): 27972809. 